\title{
THE "WHAT" AND "WHERE" OF LANDMARKS: \\ IMPACT ON WAY-FINDING AND SPATIAL KNOWLEDGE
}

\author{
A thesis submitted to \\ the Faculty of Graduate Studies and Research \\ in Partial Fulfillment of the requirements for the degree \\ Doctor of Philosophy
}

by

Shelley Roberts Kelsey

Department of Psychology

Carleton University

December 2009

(C) Shelley Roberts Kelsey, 2009 
4

\author{
Library and Archives \\ Canada \\ Published Heritage \\ Branch \\ 395 Wellington Street \\ Ottawa ON K1A ON4 \\ Canada
}

Bibliothèque et

Archives Canada

Direction du

Patrimoine de l'édition

395, rue Wellington

Ottawa ON K1A ON4

Canada
Your file Votre référence

ISBN: 978-0-494-63866-8

Ourfile Notre référence

ISBN: $978-0-494-63866-8$

\section{NOTICE:}

The author has granted a nonexclusive license allowing Library and Archives Canada to reproduce, publish, archive, preserve, conserve, communicate to the public by telecommunication or on the Internet, loan, distribute and sell theses worldwide, for commercial or noncommercial purposes, in microform, paper, electronic and/or any other formats.

The author retains copyright ownership and moral rights in this thesis. Neither the thesis nor substantial extracts from it may be printed or otherwise reproduced without the author's permission.

\section{AVIS:}

L'auteur a accordé une licence non exclusive permettant à la Bibliothèque et Archives Canada de reproduire, publier, archiver, sauvegarder, conserver, transmettre au public par télécommunication ou par l'internet, prêter, distribuer et vendre des thèses partout dans le monde, à des fins commerciales ou autres, sur support microforme, papier, électronique et/ou autres formats.

L'auteur conserve la propriété du droit d'auteur et des droits moraux qui protège cette thèse. $\mathrm{Ni}$ la thèse ni des extraits substantiels de celle-ci ne doivent être imprimés ou autrement reproduits sans son autorisation.
In compliance with the Canadian Privacy Act some supporting forms may have been removed from this thesis.

While these forms may be included in the document page count, their removal does not represent any loss of content from the thesis.
Conformément à la loi canadienne sur la protection de la vie privée, quelques formulaires secondaires ont été enlevés de cette thèse.

Bien que ces formulaires aient inclus dans la pagination, il n'y aura aucun contenu manquant. 


\begin{abstract}
When environments are novel or large in scale, acquiring and using spatial information proves to be difficult for way-finders. To ease problems associated with navigation, researchers have attempted to understand how, what type, and when environmental information is acquired. Although landmarks are argued to be one of the first and most prominent environmental features encoded by humans while way-finding, there is inconsistent empirical evidence for the impact of various features of landmarks on spatial cognition. Specifically, how they look (sensory information) and where they are located (spatial information) within the world. A 3D-desktop city-like virtual environment was used to investigate the impact two landmark features has upon wayfinding and spatial knowledge acquisition; specifically, their size (large versus small) and location (periphery versus internal) across three experiments. Experiment 1 investigated the roles landmark size plays in way-finding and spatial knowledge. Experiment 2 investigated the roles of landmark location, and Experiment 3 investigated the combined roles of the size and location as a function of increasing familiarity. Findings indicate temporal precedence of global landmarks during way-finding; however, the impact of local landmarks increased with experience, independent of landmark location. In contrast to way-finding, recalling spatial knowledge was dependent on the location of landmarks, particularly when located in the periphery. The key findings imply a distinction between landmark acquisition and use during way-finding as a function of their type and location. How and when landmarks are relied upon as a function of their type and location was determined by amount of familiarity with the environment and the spatial task. Findings are compared to the processes related to the acquisition of spatial knowledge. A
\end{abstract}


theoretical framework for the impact of sensory and spatial information provided by landmarks in way-finding and spatial knowledge is proposed. Practical recommendations for using different landmark features in the design and development of navigable virtual environments and aids are discussed. 


\section{Acknowledgements}

Early in the process of my pursuit of a higher education it became very clear to me that I could not complete this alone. Although the list of individuals that I wish to thank extends beyond the limits offered here, I would like to thank the following people for their dedication, support and belief in my efforts.

Leading my example, my Father taught me that the best things in life are worth working hard for, while my Mother taught me the importance of family and friends. Together they taught me to maintain a wonderful balance between work and play that I believe brought me to the end of this journey successfully. Mom and Dad, Sean and Chris - thank you for your financial and mental support, for your encouragement, and your unconditional love.

I would like to thank my advisor, Dr. Avi Parush for his guidance, patience, enthusiasm, support and friendship. He allowed me to grow independently as a student which in the end afforded me the maturity to develop into a confident academic and professional. Not only was he readily available, but he responded with thoughtful feedback to each and every draft of my work regardless of approaching weekends. Avi thank you for being so liberal with your time, thoughts, advice, expert guidance and knowledge. You have always made me feel that my research was of utmost importance to you. I will remember our discussions, academic and otherwise, with great fondness. Thank you for being my mentor and confidant.

I would like to extend my appreciation to the members of my committee whose time, interest and positive criticism was of great benefit to this research. In particular I would like to thank Dr. Gitte Lindgaard and Dr. Chris Herdman, both of whom were a 
great source of expert knowledge, positive guidance, and support throughout my entire tenure at Carleton.

This dissertation would not have been a success if it were not for my peers who lent their hands along the way. My friends and colleagues in the HotLab, at CAE, the undergraduates with whom I worked and my participants - I would like to thank you all for your continuous support, collaboration, participation, constant positive feedback, reassurance, presence at numerous practice talks, immediate response to assist, and great discussions. A special thank you to Del Torkornoo, Chelsea Kramer, Dr. Norm Vinson and Sarah Hildebrand - your contribution and collaboration was greatly appreciated and made this truly a collaborative effort.

It has been said that completing a $\mathrm{PhD}$ thesis can be a long and lonely journey. This in part is true, but through the support and love of those who surrounded me on a daily basis my journey was devoid of any loneliness. My friends (you know who you are) - thank you for keeping me sane and celebrating every accomplishment of mine along the way with great vigor. I could not have gotten here without each and every one of you.

Last, but most deserving of my thanks, goes to my husband Phil as he endured living with me while completing my $\mathrm{PhD}$ (please note that this is not an easy feat). Through the constant postponement of completion dates and the emotions that accompanied this. Phil - your patience, love, and encouragement have held me up and brought us here to the end. You have always reminded me to keep things in perspective and kept me laughing through the tough times. I love you and I hope that I can be half as supportive to you in all of your future endeavors as you have been to mine. 


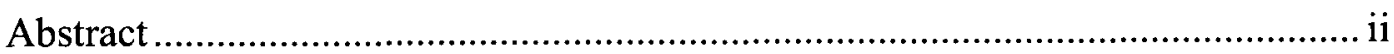

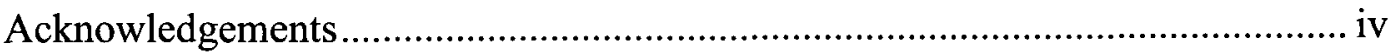

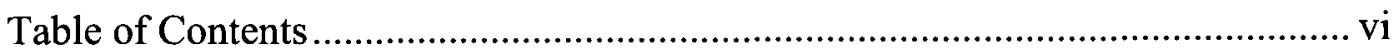

List of Tables .................................................................................... vii

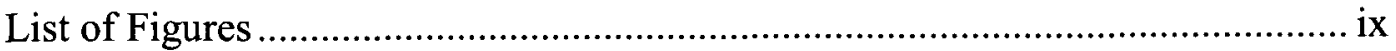

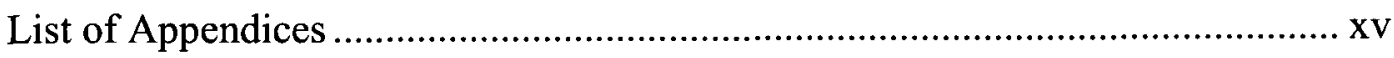

The "What" and "Where" of Landmarks: Impact on Way-finding and Spatial

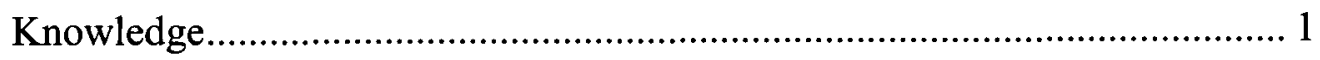

Background: Spatial Cognition........................................................ 6

Hypotheses and Research Paradigm .................................................. 38

Experiment 1: Isolating the Impact of Landmark Type ................................ 41

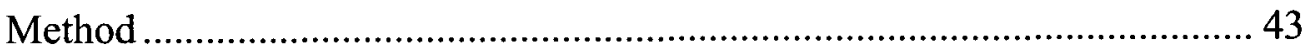

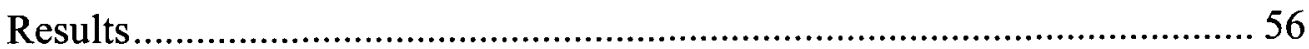

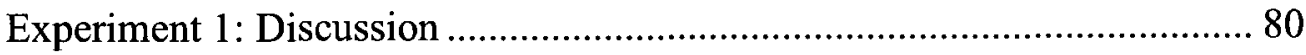

Experiment 2: Isolated Impact of Landmark Location ................................ 86

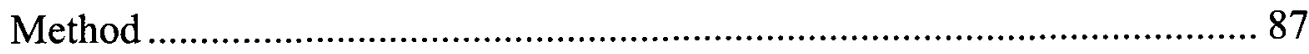

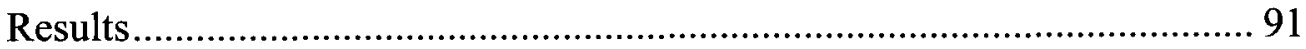

Experiment 2: Discussion .......................................................... 114

Experiment 3: Landmark Type and Location as a Function of Familiarity........ 118

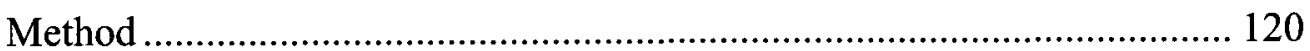

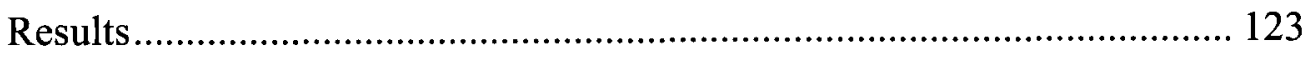

Experiment 3: Discussion ........................................................... 144 
General Discussion

Key Findings and Related Literature ……….......................................... 149

Framework: Dual Process of Landmark Knowledge ................................... 152

Implications for Theories of Spatial Knowledge Acquisition ..................... 157

Generality and Practical Implications ....................................................... 160

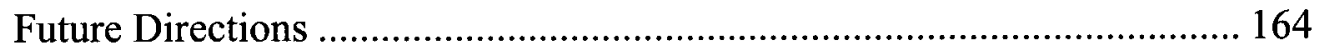


List of Tables

Table 1. Summary of Existing Research Space .......................................................25

Table 2. Summary of Current Thesis Research Space ..............................................25

Table 3. Tools Used to Create, Compile and Render the VEs.....................................43

Table 4. A Description of VE's used in Experiment 1 .............................................45

Table 5. The 5 most frequently drawn landmarks within each group. ...........................64

Table 6. Spatial Strategy Questions: Proportion of True to False Questions for Groups.....70

Table 7. A Description of Each VE Used in Experiment 2 ...........................................83

Table 8. The 5 most Frequently Drawn Landmarks by Group ......................................97

Table 9. Spatial Strategy Questions: Proportion of True to False Answers .....................104

Table 10. The 5 Most Frequently Drawn Landmarks by Group ..................................128

Table 11. Spatial Strategy Questions: Proportion of True to False Questions for Groups.131

Table 12. Elements of Framework and Associated Results .....................................148 


\section{List of Figures}

Figure 1. An example of the landmark size and location confound (drawing adapted from

Steck and Mallot, 2000). 23

Figure 2. A second example of the landmark size and location (drawing adapted from

Ruddle and Peruch, 2002).

Figure 3. An example of Navon's (1977) compound letter stimuli.

Figure 4. An overview of the research space and hypotheses for the current dissertation.

Figure 5. An overview of the three experiments for the current dissertation. 40

Figure 6. Birds-eye-view of the VE. ........................................................................... 45

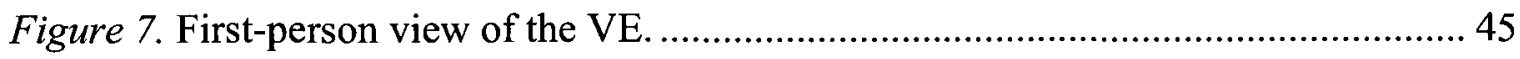

Figure 8. An overview of Experiment 1's study design............................................... 50

Figure 9. Way-finding Decision Task question........................................................... 53

Figure 10. Mean of the Natural Log of time in seconds to locate targets $(+\mathrm{SE})$ as a

function of blocks for Global and Local-only groups.

Figure 11. Mean number of targets located (+SE) as a function of blocks for Global and

Local-only groups.

Figure 12. Sketch map examples from the (a) Both, (b) Global, and (c) Local-only groups.

Figure 13. Ratio (+SE) of the number of landmarks included in map drawings for the

Global and Local-only groups.

Figure 14. Ratio (+SE) of the number of landmarks included in map as a function of landmark location for Global and Local-only groups. 
Figure 15. Ratio (+SE) of global landmarks by location included in map drawings for the

Both and Global-only groups. 66

Figure 16. Ratio (+SE) of local landmarks by location included in map drawings for the

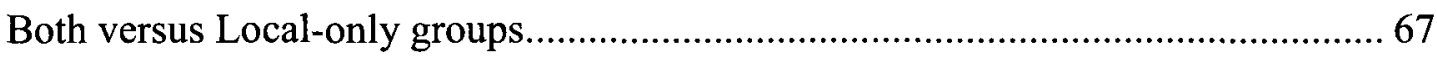

Figure 17. Relative landmark placement ratio $(+\mathrm{SE})$ for all groups. 69

Figure 18. The natural log of time (s) (+SE) spent as a function of the location collapsed across all groups. 72

Figure 19. The amount of time spent in the world as a function of location (periphery vs internal) as measured by the Ln of time in seconds ( $+\mathrm{SE})$.

Figure 20. Way-finding Decision Task ( + SE) performance for the Global and Local-only groups by landmark location. 77

Figure 21. Way-finding Decision Task (+SE) performance for Both group by landmark type and location.

Figure 22. Mean proportion of correct WDT responses (+SE) for Both and Local-only groups for global landmarks by location. 79

Figure 23. Overview of conditions experienced by the Peripheral and Internal Groups. 90

Figure 24. Way-finding performance as measured by the Ln of Time(s) (+SE) for Order 1.

Figure 25. Way-finding performance as measured by the ratio of trials to targets found $(+\mathrm{SE})$ by location for groups that received Order 1 .

Figure 26. Way-finding performance as measured by the Ln of Time(s) (+SE) for as a function of order. 95 
Figure 27. Way-finding performance as measured by the ratio of trials to targets found $(+\mathrm{SE})$ by Order.

Figure 28. Amount of time spent in the world as a function of location (periphery versus internal) as measured by the $\mathrm{Ln}$ of time in seconds $(+\mathrm{SE})$ 98

Figure 29. Ratio (+SE) of the number of landmarks included in map drawings to as a function of group at Order 1 100

Figure 30. Ratio ( $+\mathrm{SE})$ of the number of landmarks included in map drawings as a function of groups as Order 2 . 102

Figure 31. Relative landmark placement ratio (+SE) for Peripheral and Internal groups at order 1 . 105

Figure 32. Relative landmark placement ratio (+SE) for Peripheral and Internal groups at order 2 . 106

Figure 33. Map goodness rating (+SE) as a function of landmark type by Peripheral and Internal groups by order 1 . 108

Figure 34. Map goodness rating (+SE) as a function of landmark type by Peripheral and Internal groups by condition 2 . 109

Figure 35. Overall WDT performance (+SE) for Peripheral and Internal groups when exposed to Global followed by Local landmarks.

Figure 36. Overall WDT performance (+SE) for Peripheral and Internal groups when exposed to Local followed by Global landmarks. 113

Figure 37. Schematic of Experiment 3's procedure. 122

Figure 38. Mean time to locate targets (+SE) as a function of group across blocks..... 124 
Figure 39: The amount of time spent in the world as a function of location (periphery versus internal) as measured by the $\mathrm{Ln}$ of time in seconds ( $+\mathrm{SE}$ ) by group.

Figure 40. Total number of landmarks recalled (+SE) (i.e. included in sketch maps) across familiarity as a function of Experimental and Control groups.

Figure 41. Number of landmarks recalled by type (+SE) across familiarity (groups)... 128

Figure 42. Number of landmarks recalled $(+\mathrm{SE})$ as a function of type across familiarity for the control group.

Figure 43.Number of landmarks recalled by location ( $+\mathrm{SE})$ across familiarity (groups).

Figure 44. Number of landmarks recalled by location ( $+\mathrm{SE})$ across familiarity for the Control group.

Figure 45. Number (+SE) of landmarks by type and location included in participants sketch maps.

Figure 46. Number (+SE) of landmarks by type and location included in participants 1 st map drawing for the control group.

Figure 47. Relative landmark placement ratio (+SE) for control group. 135

Figure 48. WDT performance (+SE) by landmark type across level of familiarity.

Figure 49. WDT performance ( + SE) by landmark type across level of familiarity for the control group.

Figure 50. WDT performance (+SE) by landmark location across level of familiarity. 141 Figure 51. WDT performance by landmark type and location across familiarity for the control group. 143 
Figure 52. Orientation deviation in degrees $(+\mathrm{SE})$ for Both group by landmark type and location. 209

Figure 53. Mean proportion of correct $\mathrm{RDE}$ responses $(+\mathrm{SE})$ as a measure of distance performance of Both and Global-only groups for global landmarks by location. .. 213

Figure 54. JRD performance as measured by mean deviation in degrees $(+\mathrm{SE})$ as a function of landmark location at sequence 2 .

Figure 55. Orientation deviation in degrees (+SE) by landmark type across level of experience.

Figure 56. Orientation deviation in degrees (+SE) by landmark type across level of experience for "All blocks" group. 218

Figure 57. Orientation deviation in degrees ( + SE) by landmark location across level of experience.

Figure 58. Orientation deviation in degrees (+SE) by landmark location across level of experience for "All blocks" group.

Figure 59. JRD performance by landmark type and location across experience. 221

Figure 60. JRD performance as a function of landmark type and location across experience for the control group. 222

Figure 61. RDE performance (+SE) by landmark type across level of experience. ...... 223 Figure 62. RDE performance ( $+\mathrm{SE})$ by landmark type across level of experience for the control group. 224

Figure 63. RDE performance (+SE) by landmark location across level of experience. 225 Figure 64. RDE performance ( $+\mathrm{SE})$ by landmark location across level of experience for the control group. 226 
Figure 65. RDE performance by landmark type and location across experience.

Figure 66. RDE performance by landmark type and location across experience for control group. . 


\section{List of Appendices}

Appendix A. The Qualitative Analysis of Landmark Visibility ......................... 185

Appendix B. Landmarks by Name, Type, and Location ................................ 187

Appendix C. Mental Rotation Test ........................................................... 190

Appendix D. Pre and Post-Cybersickness Questionnaire .................................... 191

Appendix E. Secondary Tasks: Procedure, Questions, and Results ..................... 193

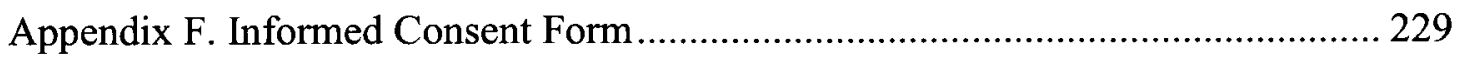

Appendix G. Demographics Questionnaire .................................................. 233

Appendix H. Experiment Instructions ..................................................... 235

Appendix I. Individual Target Information ................................................ 238

Appendix J. Way-finding Decision Task (WDT) questions ............................. 239

Appendix K. Spatial Strategies Questionnaire............................................ 253

Appendix L. Experiment 1 - Way-finding Descriptive Statistics ...................... 254

Appendix M. Experiment 1 - Sketch Maps: Descriptive Statistics ....................... 255

Appendix N. Experiment 1 - WDT Descriptive Statistics .................................. 255

Appendix O. Experiment 2 - Way-finding Descriptive Statistics....................... 257

Appendix P. Experiment 2 - Sketch Maps: Descriptive Statistics.......................... 259

Appendix Q. Experiment 2 - Spatial Strategy Questionnaire Descriptive Statistics 261

Appendix R. Experiment 2 - WDT Descriptive Statistics .................................. 262

Appendix S. Experiment 3 - Way-finding Descriptive Statistics ........................ 263

Appendix T. Experiment 3 - Sketch Maps: Descriptive Statistics........................ 265

Appendix U. Experiment 3 - WDT Descriptive Statistics ................................ 266 
The "What" and "Where" of Landmarks: Impact on Way-finding and Spatial Knowledge

Way-finding is the process of getting from one point to another in an environment by acquiring and utilizing spatial and environmental information. These tasks prove to be more difficult in a novel or large-scale environment compared to more familiar or smaller environments (Edwards \& Hardman, 1989; Elvins, 1997; Rumpradit \& Donnell, 1999). To understand the problems associated with way-finding researchers have investigated how we might acquire and use various types of spatial information.

One type of spatial information investigated is landmarks. Landmarks are argued to be one of the most prominent environmental features encoded and utilized while wayfinding. Their significance to way-finding is supported by a large body of research (Darken \& Sibert, 1996; Elvins, 1997; Elvins, Nadeau, Schul, \& Kirsh, 2001; Parush \& Berman, 2004; Siegel \& White, 1975; Tversky, 1991, 2003; Tversky, Franklin, Taylor, \& Bryant, 1994). Only a small portion of this literature addresses the specific features of landmarks and their associated significance for way-finding (e.g. (Steck \& Mallot, 2000; Vinson, 1999). Considering the well-documented impact of landmarks on way-finding, acquiring, and recalling spatial knowledge, I argue that further investigation is needed to identify which particular features of landmarks are critical to effective way-finding.

Landmark features can be classified by various visual or other sense-based characteristics such as colour, texture, sound, or smell (Burt, Holland, \& Guilford, 1997; Gunther, Kazman, \& MacGregor, 2004; Strasser, Bingman, Ioale, Casini, \& Bagnoli, 1998; Vinson, 1999). A commonly used categorization is based on a landmark's visual distinction: its size or spatial footprint. Through observations and interviews with way- 
finders, Lynch (Lynch, 1960 ) was the first to distinguish between large, prominent landmarks and smaller, less prominent ones. More recent research defines these landmarks as "global" and "local" (Steck \& Mallot, 2000; Steck, Mochnatzki, \& Mallot, 2003). Global landmarks are objects that are visible within a large area in the environment; they provide a frame of reference or compass-like information, help organize and provide structure to the environment, and should not change visually when the observer moves a small distance away (Makany, Karadi, Kallai, \& Nadel, 2002; O'Keefe, Burgess, Donnett, Jeffery, \& Maguire, 1998; Steck \& Mallot, 2000). Wayfinders may plan and execute their movements in a manner that keeps global landmark bearings constant, or have them change in well-defined ways (Steck \& Mallot, 2000). Local landmarks are objects that are visible within a localized area and are used at specific points while making way-finding decisions (Ruddle, Payne, \& Jones, 1997; Steck \& Mallot, 2000). Research shows that navigation by way of local landmarks relies on a sequence of intermediate goals defined by these landmarks and therefore are linked to our knowledge of the route (Klippel \& Winter, 2005; Kuipers, 1978, Steck \& Mallot, 2000; Winter, 2003). This body of literature supports the argument that way-finding, representation, and recall of environmental information may be affected differently depending on the size or visibility of landmarks.

Based on a critical review of the aforementioned research on global and local landmarks it appears that while studies have focused on the two sizes of landmarks, findings may be confounded by the location of the landmarks in the environment. In previous research global landmarks were typically placed in the outer boundaries of the 
environment while local landmarks were placed internally. This implicit confound between landmark size and location raises doubts regarding the interpretation and implications drawn from the results. The reported effects could be due to landmark size, location, or a combined impact of both features. Considering where a landmark is located is an obvious spatial feature of each landmark as it may have a significant impact on wayfinding and the acquisition of spatial knowledge. Therefore, the need to separate the impact of the features of size and location was established as one of my central goals.

This dissertation explored how landmark size and location in isolation and in combination impacts way-finding and spatial knowledge acquisition. Some specific questions I had were: Can landmark size and location be isolated and still play a role in the acquisition of spatial knowledge? In contrast to previous research, are global landmarks situated internally acquired and used in the same way as those located in the periphery? Are local landmarks relied upon or acquired differently if they are placed in the periphery or "boundaries" of an environment? And in general, how are global and local landmarks acquired and what roles do they play within way-finding and during spatial knowledge acquisition?

Behavioural, developmental, and neurological studies provide converging evidence for the different roles that global and local landmarks play in spatial knowledge acquisition and way-finding (Burgess, 2008; G. Evans, Brennan, Skorpanich, \& Held, 1984; GouldBeierle \& Kamil, 1996; Save \& Poucet, 2000). The most prominent theory of how spatial knowledge is acquired is that it occurs in a consecutive stage-like fashion. The stages begin with acquiring landmark knowledge, followed by route, and finally 
survey knowledge; all of which will be discussed in detail in the following chapter (Siegel \& White, 1975). Other theories suggest that global properties, or survey knowledge, can be acquired earlier in the process followed by the details or in parallel with the details and other spatial information (Hunt \& Waller, 1999); Montello, 1998; (Parush \& Berman, 2004). If the order in which global and local landmarks are acquired is demonstrated a better understanding of spatial knowledge processing can be gained. Specifically, if global landmarks are learned prior to or in parallel with local, along with their associated spatial knowledge, some level of survey knowledge may be acquired prior to the details. This is suggested as global landmarks are assumed to be associated with survey knowledge. If local landmarks are learned prior to global, along with their associated spatial knowledge, it could support the traditional stage-like theory of spatial knowledge acquisition.

The overall objective was to investigate the isolated and combined impact of landmark size and location on way-finding performance and spatial memory tasks. Theoretically, the goal of this dissertation was to provide insight into the processes used to acquire spatial knowledge based on roles that landmark size and location may play. Practically the goal was to be able to provide design guidelines regarding how to utilize different landmark types in designing and developing navigable virtual environments to facilitate better way-finding performance. The specific research questions of this dissertation include:

1. Do large and small landmarks have equivalent impact independent of their location, or does one size have precedence over the other? 
a. Are both essential for way-finding or acquiring spatial knowledge?

2. Do the locations of landmarks have equivalent impact independent of their size, or does one have precedence over the other?

a. Are both essential and is either way-finding or acquiring spatial knowledge possible without the other?

3. Does the size and location of landmarks in combination play a role in how they are attended to, acquired, and integrated into a cognitive map across time, or does one take temporal precedence?

To investigate differences between global and local landmarks a desktop virtual environment (VE) was used. This VE contained global and local landmarks that were located peripherally and internally. In an attempt to sustain ecological validity, the environment was created as an open-air "realistic" urban desktop 3D virtual environment. The research questions were investigated using the same VE across all three experiments. Experiment 1 focused on the roles landmarks may play by their size in isolation (i.e., global landmarks only and local landmarks only) while way-finding and acquiring spatial knowledge. Experiment 2 focused primarily on the roles landmarks by their location may play in way-finding and spatial knowledge acquisition. Experiment 3 investigated the roles of size and location in combination as a function of experience in order to gain insight into how they are attended to, acquired, and integrated into a cognitive map across experience.

Review and discussion of the main bodies of literature used to support the research questions are presented in the following chapters. First, an overview of spatial 
cognition and the processes involved in acquiring spatial information to way-find is presented to provide relevant background information. Second, a review and critique of relevant landmark research is provided. Finally, a synthesis of the literature and hypothesized roles that landmarks may play by their size and locations is presented.

\section{Background: Spatial Cognition}

The field of spatial cognition is concerned with how spatial information is acquired, organized, maintained, and used. By way of acquiring spatial knowledge over time humans and animals have the ability to find their way through environments in order to reach specific unobservable goals without the use of navigation aids. Two types of abilities are involved in moving successfully through real and VEs: navigation and orientation. Navigation consists of two linked abilities: locomotion and way-finding ( $R$. P. Darken \& Peterson, 2002). Locomotion is the physical motor element of navigation and involves the ability of getting from "here" to "there". Way-finding is the cognitive element of navigation and includes abilities such as planning efficient routes, finding specific locations, and recognizing destinations when reached (Elvins, 1997). Finally, orientation requires the knowledge of where one is with respect to objects and other cues (Satalich, 1995).

To navigate and orient ourselves within an environment requires the development and use of a mental representation of the area. This is accomplished by constructing an internal image of the spaces encountered and being able to recall the image at a later time. This internal representation is sometimes referred to as a mental or cognitive map (Tolman, 1948). Throughout this dissertation I will refer to this internal representation as 
a cognitive map. Support for the neural presence of cognitive maps is found within neuropsychological research, where the maps are encoded in the cells of the hippocampus of humans (Maguire et al., 1998; Maguire, Burgess, \& O'Keefe, 1999; Maguire, Frith, Burgess, Donnett, \& O'Keefe, 1998; Maguire et al., 2003) and animals (Burgess, 2008; O'Keefe, et al., 1998). While the ability to form a cognitive map is not typically debated, the manner by which cognitive maps are acquired, constructed, and represented is still disputed both behaviourally and neurally. The body of literature regarding cognitive maps is important to this dissertation as one of my goals is to understand what roles landmarks play in forming a cognitive map based on their size and location.

\section{Theories of Spatial Knowledge Acquisition}

The ability to successfully navigate based on what is known about an environment depends on the spatial knowledge acquired and the cognitive processes involved in organizing, storing, and retrieving this information from our cognitive maps. One of the first and foremost theories of how cognitive maps are developed is that of Siegel and White (1975) who argued that the development of how we learn the layout of an environment involves acquiring three types of knowledge in a sequential way: landmark, route, and survey knowledge (Thorndyke \& Hayes-Roth, 1982). This classification of spatial knowledge is argued to form in a progressive and sequential manner and is referred to as the LRS theory (Lynch, 1960; Siegel \& White, 1975; Yeap \& Jefferies, 2001). Landmark knowledge is simply a memory for an object or a specific scene that is stored and later recognized when perceived (Montello, 1998). To have route knowledge is to have knowledge of links or chains of landmarks that are located along an 
experienced path and to be able to associate them with a particular action, such as "turn right at landmark x" (Montello, 1998). Survey knowledge, the last and most complex type of spatial knowledge to be acquired, includes distance and direction information and allows the navigator to find places that are not perceptually observable affording one to plan alternative novel routes (Bosco, Longoni, \& Vecchi, 2004; Montello, 1998). To have survey knowledge of an environment is interchangeable with the term 'cognitive map'. Each implies a map-like representation of the environment that integrates routes into a network of relationships between locations and features of the environment (Maceachren, 1992b; Thorndyke \& Hayes-Roth, 1982).

Evidence in favour of Siegel and White's (1975) LRS theory exists but is not robust (Appleyard, 1970; Maceachren, 1992b). Appleyard (1970) analyzed sketch maps across level of familiarity and found that those who were new to the city drew more paths compared to those who had lived in the area long term. Devlin (1976) also investigated cognitive maps across the level of familiarity by comparing sketch maps of Navy wives who were recently transferred to a new city at two weeks and again at three months. It was found that their first and second maps were both quite accurate but not qualitatively different. This finding led Devlin (1976) to argue that changes in construction of cognitive maps must occur early on in the process of spatial knowledge acquisition.

Different results may have been found in both studies if the time at which knowledge was measured was at shorter or longer amounts of familiarity with the VE. In general, studies of cognitive map construction over time such as Appleyard (1970) and Devlin (1976) do not provide strong evidence in favour for the LRS model. In fact, their results imply that 
landmark knowledge may be more complete than route and survey in the early stages of forming a cognitive map, but not necessarily learned prior to route or survey knowledge. There is some evidence that survey type representations can be acquired following a single exposure of a route in a novel environment (Rovine \& Wiseman, 1989). This finding indicates that survey representations may not follow a sequential landmark-toroute-to-survey process as suggested in the LRS model.

A contrasting body of research suggests that aspects of landmark, route, and survey knowledge may be acquired in parallel (Couclelis, Golledge, Gale, \& Tobler, 1987; Jefferies \& Yeap, 2001; Kuipers, 2000; Maceachren, 1992a; Montello, 1998; Montello \& Pick, 1993; Ruddle \& Peruch, 2004; Spencer, Simmering, \& Schutte, 2006; Yeap \& Jefferies, 1997). While the stage-like LRS theory may not have strong empirical support, the distinction between the three types of knowledge that this model offers is extremely useful as the rate at which each develop may be affected by different factors, such as the type of landmarks available (Ruddle \& Peruch, 2004). In general, two opposing models, the stage-like LRS model and the more "in parallel" model, were presented and critiqued. Further, the three different types of spatial knowledge, landmark, route, and survey were reviewed. These facets of spatial cognition are central to my dissertation and will be referred to throughout. In the next section I review and critique research related to the specific roles of landmarks.

\section{The Role of Landmarks}

The term "landmark" is described and defined in numerous ways across various research fields including psychology, geography, and urban planning (Golledge, 1999; 
Lynch, 1960 ; Steck \& Mallot, 2000; Vinson, 1999). However, for the purpose of this research, a landmark is defined as an object attended to within the environment that aids the user in navigating, understanding or recalling the space (Elvins, 1997; Lynch, 1960 ; Presson \& Montello, 1988). Any object in an environment can act as a landmark; however, not all objects are landmarks. There can be numerous objects within an environment but to make the object an actual landmark, they must be attended to and aid the user in some helpful way. Landmarks have been shown to be very effective in supporting navigation and orientation in real (Elvins, 1997; Lynch, 1960; (May, Ross, Bayer, \& Tarkiainen, 2003) and VEs (Darken \& Peterson, 2001; Darken \& Sibert, 1993; Parush \& Berman, 2004), while performance is significantly degraded in their absence (Darken \& Sibert, 1996, Parush \& Berman, 2004).

Neither the stage-like theory nor the "in parallel" theories of cognitive map construction address how different types of landmarks may be acquired. If we look into the process in more depth, perhaps specific types of landmarks are learned prior to other types. It can therefore be argued that the process of acquiring landmarks first - prior to route and survey knowledge - may be less "fixed". For example, global landmarks may be attended to and acquired prior to local landmarks as we try to provide some structure to our spatial knowledge of the environment. Or perhaps we acquire local landmarks prior to global in order to complete a task to get from point $\mathrm{A}$ to point $\mathrm{B}$ and have no need to acquire a higher level of spatial knowledge.

One theory of spatial knowledge acquisition that gives landmarks centre-stage is the anchor-point hypothesis (Couclelis, Golledge, Gale, \& Tober, 1987). This hypothesis 
argues that landmarks may provide the foundation for spatial knowledge acquisition and representation. Although landmarks may be acquired first, in agreement with the LRS model, it may be that specific types of landmarks are acquired first based on the information that they provide. According to the anchor-point hypothesis, salient cues are used to "anchor" each region of a space. These regions, based on the anchors, are then more easily represented as a cognitive map. Authors also suggest that anchoring occurs quite early in the spatial knowledge acquisition process (Couclelis, et al., 1987; Wiener, Schnee, \& Mallot, 2004). According to the working definition these researchers are referring to, landmarks can be classified as global. Therefore, global landmarks acting as anchors or reference points may help to construct survey knowledge with little familiarity. Our understanding of how and when global and local landmarks are acquired and integrated into cognitive maps may provide insight into which model of spatial knowledge acquisition is most accurate.

Although it is well known that landmarks are useful for way-finding, there is a scarcity of research focusing on the different features of landmarks based on the information that they may provide or the roles that they may play. Understanding such features can provide insight into how and when they are acquired, integrated into cognitive maps, and used while navigating and orienting oneself. In turn, this knowledge can be applied to the design of VEs, navigation aids and modeling and simulating human behaviour. Presented next are the various categorizations of landmarks, and a discussion and critique of the relevant findings of landmark use while way-finding. 


\section{Global and Local Landmarks}

Thus far, I have presented 'global' and 'local' as one common categorization of landmarks. This categorization is based on object size and visibility, and the different types of information that each may provide. Global landmarks are defined as objects visible from a distance, and are hypothesized to provide orientation information by acting as reference points (Ruddle \& Peruch, 2004); Steck \& Mallot, 2002). Local landmarks are defined as objects visible within a localized area and provide information at points in the environment when making way-finding decisions (Glenn \& Chignell, 1992; Nielsen, 1995; Steck \& Mallot, 2002). Based on these definitions, it can be argued that access to both global and local landmark information can facilitate navigation. .

The distinction made between global and local landmarks is not only of concern to psychologists and human computer interaction (HCI) specialists, but also to fields such as geography, urban planning, and architecture (Lynch, 1960; (Werner \& Long, 2003). Lynch (1960), a geographer and urban planner, observed and interviewed residents of various cities. Through observations and collection of anecdotes he concluded that residents attend to and recall certain features to help organize and navigate their cities. One of the first features Lynch argued to be important were landmarks. He further categorized landmarks that are visible from a distance (global or distal landmarks) and those that can be seen from a localized area (local or proximal landmarks). Lynch argued that distant landmarks are used mostly by naïve travelers as reference points, whereas local landmarks are more personal and provide information in making way-finding decisions. Although Lynch's work is compelling, well respected, and frequently cited, his 
findings were based on anecdotal evidence, observations and interviews, without objective empirical performance data. This thesis attempts to quantify his findings from a more empirical perspective.

Further justification for the importance and relevance of investigating global and local landmarks comes from the fields of developmental (Heth, Cornell, \& Alberts, 1997; Jansen-Osmann \& Wiedenbauer, 2006), neural (Save \& Poucet, 2000), and comparative psychology (GouldBeierle \& Kamil, 1996). It has been found that global and local landmarks processing may be mediated by different neural systems (Save \& Poucet, 2000) and may be acquired differently depending on the stage of development (Heth, Cornell \& Alberts (1997). For example, a study by Heth, Cornell \& Alberts (1997) found that 12 year old children attend to and recall a higher percentage of global landmarks compared to 8 year olds. It has also been found that children are less capable at judging the potential value of global landmarks as helpful cues (Allen, Kirasic, Siegel, \& Herman, 1979). Within comparative cognition (Clark's nutcrackers) birds are found to be more sensitive to global landmarks compared to local when exhibiting caching behaviour (GouldBeierle \& Kamil, 1996). Therefore, previous research uncovers neural and developmental distinction between global and local landmarks implying a need to further investigate landmarks based on this categorization. This is just an example of the globallocal related findings as they will be discussed in greater detail in following sections. The global-local distinction reviewed thus far is based primarily on the visual features of landmarks (i.e., visibility or size). Other categorizations of landmarks have been 
proposed based on visual or other characteristics. These are outlined next to provide a comprehensive overview of alternative landmark category schemes.

Hirtle and Sorrows ((Hirtle \& Sorrows, 1998) argue that landmarks can be categorized based on their visual, cognitive, and structural dimensions, with each playing a different role in 2D and 3D virtual navigation. The authors describe a visual landmark as one that stands out in appearance, with such characteristics as its location, amount of contrast against the background, or its own visual characteristics. A cognitive landmark is one that stands out because of its cultural or personal importance, such as your own house, place of work, or favorite restaurant. A structural landmark is given importance because it of its role or location within the structure of the space, for example, a town square located in the city's centre. These three characteristics are claimed to exist dependently; a "good" landmark, according to Hirtle and Sorrows (Hirtle \& Sorrows, 1998), is one that contains characteristics from all three categories. The authors state that these three categories thoroughly encompass all landmarks in both real and electronic (i.e., websites) spaces. A dissertation followed by Sorrows (2005) confirming that landmarks that are rated high on visual, structural, and cognitive characteristics within web pages were frequented more, and their recall was superior. Although this categorization of landmarks seems sound, it should be noted that the research conducted was on web-page navigation and has yet to be generalized to a 3D environment.

Stankeiwicz and Kalia (2007) also attempted to define the properties of landmarks and suggest that a "good" landmark is one that is persistent, salient, and informative. A landmark, according to the authors, is persistent if it exists in the same location upon 
repeated visits, salient if it is detectable and identifiable, and informative if it provides information about one's location and what action to take. They propose that the more permanent, salient and informative the landmark is, the more likely it will be attended to, encoded, and recalled. Although these properties are important and perhaps even measurable to some degree, they do not provide any specific visual or spatial characteristic for which physical characteristics a "good" landmark would demand.

In summary, there are landmark categorizations based on numerous influential features, which reflect the complexity of defining them. The visual features are not discussed in detail, or are inconsistent across existing categorizations. As it is outside the scope of this thesis to investigate all of the categorizations of landmarks, this research will focus on the global-local categorization as it has the richest support spanning various fields that, at this time, provide parallel findings more often than not. Research that provides evidence of the different impact global and local landmarks play within wayfinding and spatial knowledge acquisition is discussed next.

\section{Impact of Global and Local Landmarks}

Numerous studies have investigated the impact landmarks have on navigation and orientation (G. Evans, et al., 1984; Ruddle \& Peruch, 2004; Steck \& Mallot, 2000). However to date, few have attempted to explicitly investigate the role of global and local landmarks in humans (Evans et al., 1984 and Steck \& Mallot, 2000 are known exceptions). As such, this section discusses previous research on the individual impact of local and global landmarks. 
Impact of Local landmarks. The claim that local landmarks are closely associated with route related tasks and associated knowledge is supported in the literature (Aginsky, Harris, Rensink, \& Beusmans, 1997; Parush \& Berman, 2004; Tlauka \& Wilson, 1994). Aginsky et al. (1997) studied the strategies people use to learn routes in a driving simulator. Researchers had people learn a route that provided only buildings placed along the route as landmarks. Participants' sketch maps (i.e., maps drawn by participants following the experimental tasks) revealed that the landmarks recalled were those placed at intersections or points of direction change. To investigate the effect of local landmarks on way-finding, their locations were manipulated by switching them along routes (Aginsky et al., 1997). People who based their way-finding decisions on landmarks placed along the route were not able to integrate the routes into any survey type representation within their sketch maps. Therefore, the authors argued that although most participants had acquired some route and survey knowledge, several preferred a strategy based mostly on the landmarks provided, while others built a survey representation from the beginning. Given that those who developed survey knowledge were not affected by landmark removal, it could be speculated that landmarks that are small and are located along routes are associated with route knowledge, and to a lesser extent with survey knowledge.

A further study presented adults with a video of a route to investigate the effect of "external" and "internal" landmarks on route memory (G.W. Evans, Skorpanich, Garling, Bryant, \& Bresolin, 1984). The external landmarks (i.e., referred to as global) were tall structures placed in the line of sight and visible from a distance. Internal landmarks (i.e., 
referred to as local) were smaller landmarks placed within the immediate surroundings along the route. Both internal and external landmarks were included to investigate Lynch's (1960) proposal that external landmarks would facilitate survey knowledge by aiding way-finders with direction and orientation. Memories for landmarks by their type were revealed via sketches of routes and photo manipulations. No significant differences were found between internal and external landmarks; however, people did have a slight memory preference for local landmarks. Consistent with previously discussed findings (Aginsky, et al., 1997), it can be argued that the internal landmarks are more closely associated with route knowledge and route related tasks. Evins et al. (1984) suggested that a worthy future research direction would include a more rigorous investigation of these landmark types with dynamic way-finding behaviour versus the video presentation of scenes used in their research. This recommendation was implemented into the research paradigm used in this thesis.

Further evidence for the role that local landmarks play in route knowledge can be found in a study of landmark use in a 3D building environment. Parush and Berman (2004) investigated how the type of aid - map versus route depictions - impacts navigation with and without local landmarks. Included landmarks were typical home and office objects such as plants, paintings, and furniture. Results showed that when unfamiliar with the VE, navigation actually took longer with local landmarks. However, with familiarity the difference between way-finding with and without local landmarks was no longer significant. The authors suggested that this could be due either to participants spending more time learning the landmarks and their locations in the 
beginning, or gradually ignoring them later in the task. The navigation aid provided further insight into participants' spatial ability. The way-finding of the group who learned the VE with local landmarks were not impacted upon removal of the aid. This suggests that participants who navigated with local landmarks may have acquired better route knowledge of the space and therefore suffered less upon the removal of the aids.

Although local landmarks may aid in way-finding tasks, they may not aid in tasks related to orientation and survey knowledge. (Jansen-Osmann \& Fuchs, 2006) investigated the impact of local landmarks on way-behaviour and spatial knowledge acquisition. Their findings indicated that children and adults use local landmarks for close location way-finding but they did not provide any additional benefit for orientation or spatial knowledge acquisition. The authors argued that although local landmarks may be used as navigational aids they may not be important for spatial knowledge acquisition. Authors suggested that other categories of landmarks, such as global versus local, be investigated for their impact on spatial knowledge acquisition and orientation (JansenOsmann \& Fuchs, 2006). Overall, findings from the reviewed research involving local landmarks support the claim that they are closely associated with route tasks. Next, findings that provide an indication for the role of global landmarks are reviewed.

Impact of Global Landmarks. It is suggested that global landmarks provide information related to survey knowledge. Ruddle and Peruch (2004) examined how defining the boundaries of VEs would affect navigation and cognitive map construction. To do so, global landmarks, in the form of tall posts, were added to the corners of a virtual maze in order to define the boundary and provide global orientation information in 
hopes of facilitating organization of the space into regions. This condition was compared to a second condition where the perimeter of the maze was visually defined by a 2 dimensional border. They hypothesized that global landmarks would facilitate faster survey knowledge acquisition compared to the perimeter condition because in addition to marking the boundary, the landmarks can also be used as orientation information and help structure the maze. In addition, the perimeter and global landmark condition differed in that the global landmarks can be seen from most places within the space, whereas the perimeter cannot. It was found that participants' searching performance was worse when either of these cues were absent. However, differences between the perimeter and global landmark conditions were not found. Authors suggested that both the marked perimeter and global landmarks provided the same type of information.

Stankeiwicz and Kalia (2004) compared two types of landmarks with different perceptual properties; structural and object landmarks. Structural landmarks are defined as cues that provide structure to an environment, such as hallways or T-junctions. Object landmarks are defined as visual objects within the environment that are independent of its structure, such as art on the wall or a fountain in the park. Structural and global landmarks are similar in that they may both provide structure and organization to the space, as findings from Ruddle \& Peruch (2004) suggest. But different in that a structural landmark does not typically have the defining characteristic of visual saliency from far away, a determining feature of global landmarks. To date, it has not been argued whether or not structural landmarks would be considered a type of global landmark or vice versa. However, working with the current definition of a global landmark, they would be 
considered different from structural as the information is unavailable from a distance. Stankweiwicz and Kalia (2004) found a bias towards learning and recalling structural landmarks over object landmarks. Although the authors did not offer an explanation, two reasons are proposed. First, the structure of a building has more persistence and is less likely to change over time. According to the authors (Stankeiwicz \& Kalia; 2004) the more persistent the landmark, the better landmark it will be. The second is that the structure of the building may be more informative than a picture on a wall in that it actually directs us as to where to make navigational decisions such as turn or go straight, and not just inform where one is within the building. Again, according to the authors a landmark that is more informative is better. It can be argued that if hallways are used to represent structure may be defined as a path not a landmark, and therefore comparing a path to a landmark is inconsistent in terms of the type of information each is providing the way-finder.

Significance of the distinction between global and local landmarks. What is known about the environment and how we move throughout it depends on the spatial knowledge acquired and the cognitive processes involved in organizing, storing, and retrieving this information. Overall, it is agreed that humans and animals form some type of internal representation of environments. However the way cognitive maps are formed, the type of information that is attended to and included, and the way they are represented is still not well understood. The lack of support for the LRS model of Siegel and White (1975), or as I argue the lack of depth, paves the way for the argument that the presence 
of different landmark features (i.e., size and location) could influence the formation of one's cognitive map.

As the LRS model does not distinguish between types of landmarks and their acquisition, it is open to hypothesize that these environmental features could be learned in one order or another. The anchor-point hypothesis supports the claim that global landmarks may be learned prior to local as they help to organize the space before learning any routes. If global landmarks are learned prior to local and associated with particular regions of space, this could provide evidence for the different roles these landmarks play, based on their size, in cognitive map construction. First, as stated previously, global landmarks are argued to provide information associated with distance, direction, frame of reference, and other higher-level survey related knowledge. Therefore, acquiring information about global landmarks early in the process of spatial knowledge acquisition would suggest that the development of cognitive map formation does not follow a stagelike process in the way that Siegel and White (1975) had once proposed. I will attempt to provide evidence for the order, or lack thereof, in which global and local landmark knowledge is acquired and integrated into ones' cognitive map. Results will provide insight into what type of knowledge is acquired in the presence and absence of global and local landmarks.

\section{Global and Local Landmarks: Summary and Critique}

Thus far I have introduced the research related to different features of landmarks and their impact on spatial cognition. Overall, there is a widespread consensus that landmarks have an impact on way-finding and the acquisition of spatial knowledge to 
some degree. However, there is no consensus on a definitive and unambiguous categorization of landmarks that will allow a more conclusive theoretical and practical understanding of landmark features and their resulting impact. Previous research has focused primarily on the visual features of landmarks. The distinction between global and local landmarks was found to be appropriate for many studies, and support for this categorization can again be found in comparative psychology and neuropsychological areas of research. A global landmark is usually large and visible from far away, whereas local landmarks are smaller and visible only within a localized area along a route. Based on the definition of global landmarks, their role is to serve as reference points and provide information that helps to structure the space, and are assumed to be associated with survey knowledge. The role of local landmarks is to provide information regarding way-finding decisions while traversing routes, and is argued to be associated with route knowledge. Based on the definitions and studies focusing on such features, it is clear that the global versus local distinction in landmarks has a critical impact on spatial cognition. Although there is support for this distinction between the two features based on size, following a critical analysis of the literature there is a potential conflict which leads to the tentative use and interpretation of the findings at face value. This conflict is discussed next.

\section{Confound: Size or Location?}

Upon careful examination of the literature reviewed thus far, two distinct features of landmarks - the size or visibility of the landmark, and its location - are embedded within the operational definitions of the two landmark types. For example, the global 
landmarks used in Steck and Mallot's (2000) research were located on the periphery of the environment, while the local landmarks were internally located (see Figure 1).

Another example of this confound can be found in Ruddle and Peruch's (2004) experimental world where the four global landmarks investigated were bound to the four outer corners of the world (see Figure 2).

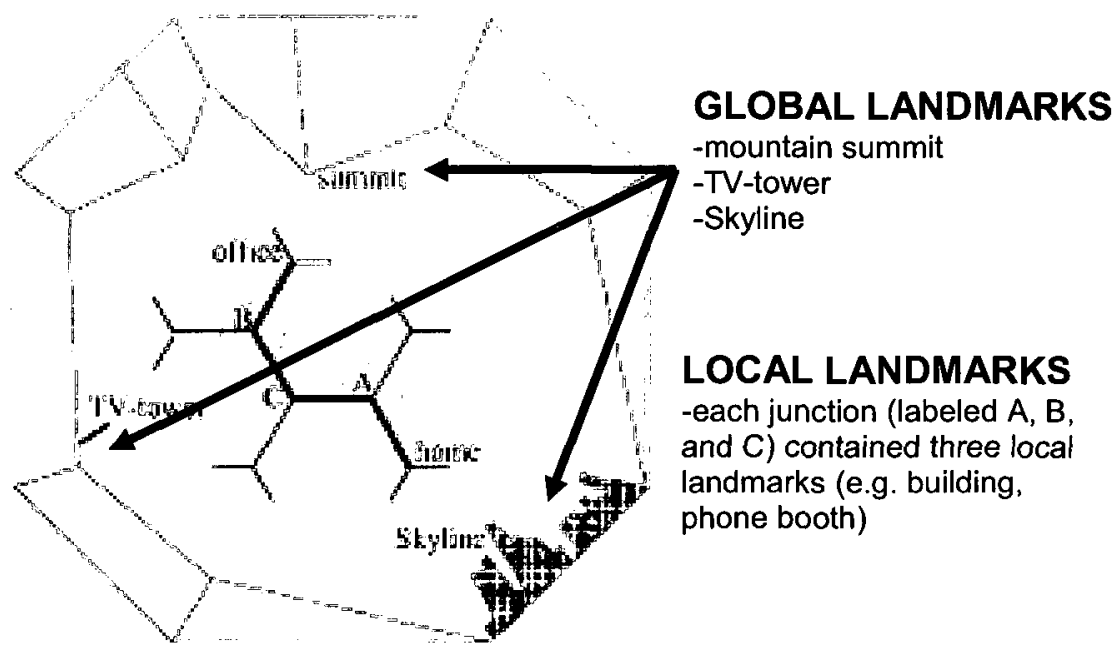

Figure 1. An example of the landmark size and location confound (drawing adapted from Steck and Mallot, 2000). 


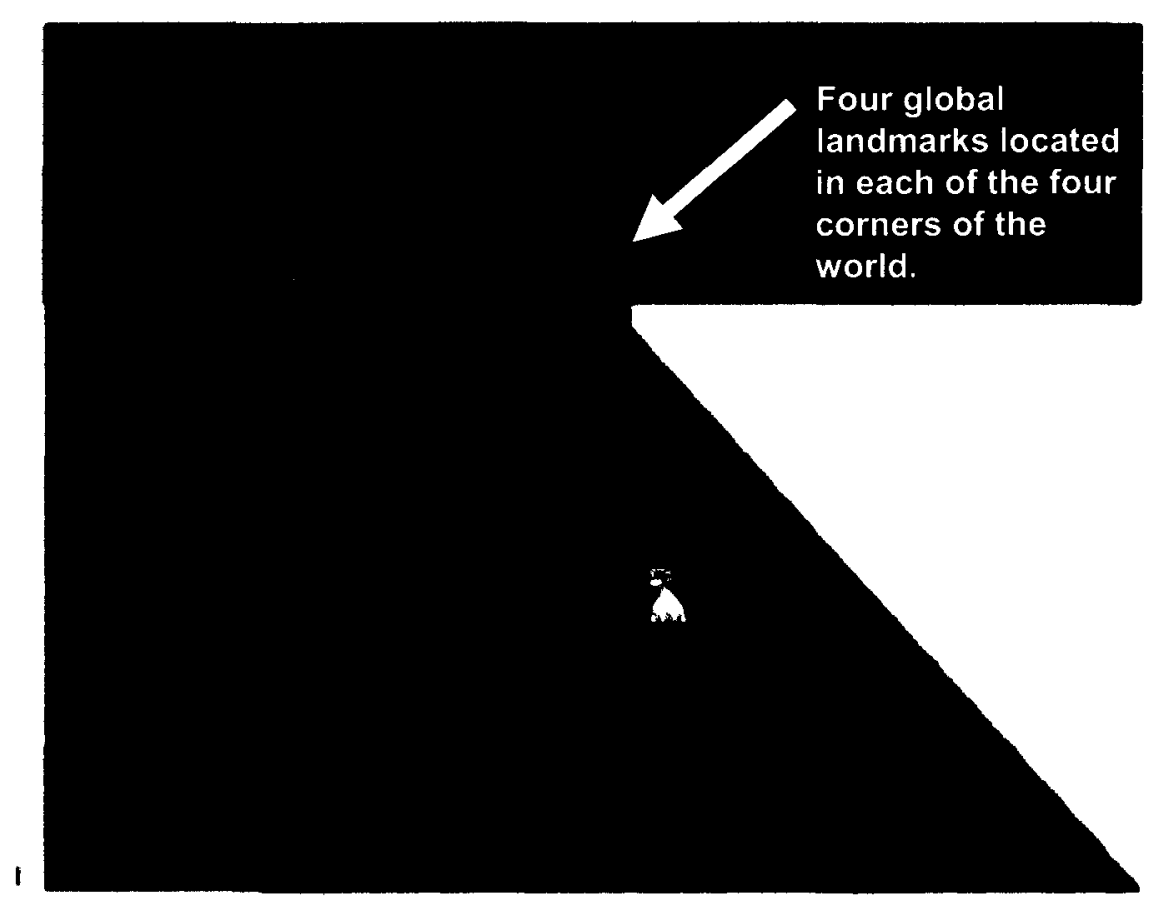

Figure 2. A second example of the landmark size and location (drawing adapted from Ruddle and Peruch, 2002).

Based on this trend within previous research, I argue that the distinction between global and local landmarks has entangled two different features: size and location. This confound is summarized in Table 1. Consequently, if we are to understand and apply theoretically and practically - the impact of various features of landmarks on spatial cognition, we are faced with a conflict: are the effects we have observed due to the impact of size and visibility, the impact of location, or due to some combined impact of both? With respect to how landmarks impact the acquisition of spatial knowledge, what impacts potential precedence for one over the other - size or location? For consistency, the terms "global" and "local" will be referring to landmarks to based on their size only (large/small), that is, independent of their location (periphery/internal or distal/proximal). 
Referral to global or local landmarks in combination with a specific location it will be explicitly stated.

\section{Table 1}

Summary of Existing Research Space

\begin{tabular}{lll} 
Landmark Type & Size & Location \\
\hline Global & Large & Distal \\
Local & Small & Proximal \\
\hline
\end{tabular}

Given the reviewed literature it is suggested that the existing framework entangles the two features: size and location. Although it is believed that there is adequate research to hypothesize the roles of each type, questions arise such as: can large landmarks that are situated internally, that is, located away from the periphery or boundary of a given environment, be acquired and used in the same way as the previously defined "global" landmarks in terms of providing a frame of reference, distance information, or for orientation? Are smaller landmarks typically confined, experimentally at least, to the internal space relied upon or acquired any differently when they are placed in the periphery or "boundaries" of an environment? A general interest to this dissertation is whether it is the size of landmarks, the location, or a combination of the two features that mediate these roles for way-finding and spatial knowledge acquisition. These questions provide the basis for the current research space that will be investigated and can be referred to in Table 2. Within Table 2the bolded text represents the landmark features that have been neglected in previous research. 
Table 2

Summary of Current Thesis Research Space

\begin{tabular}{cccc} 
& & \multicolumn{2}{c}{ Size } \\
& & Large & Small \\
\cline { 3 - 4 } Location & Peripheral & Global-Peripheral & Local-Peripheral \\
& Internal & Global-Internal & Local-Internal \\
& & & \\
\cline { 3 - 4 } & &
\end{tabular}

Overall, researchers agree that navigation without landmarks is extremely difficult and disorienting. However, there is no consensus on a definitive, unambiguous categorization of landmark types and their associated roles. There is a tendency within this area of research to discuss the different features of landmarks and their associated functions in a non-definitive way. Researchers believe that the strongest defining feature of a global landmark is that it is visible from far away, whereas local landmarks are visible only within a localized area along a route with no mention of the spatial information associated with each. This leaves unanswered questions regarding the roles of global and local landmarks. I suggest that the roles of global and local landmarks based on their location are deserving of empirical attention and may be pertinent for use by navigators.

Findings discussed in this chapter provide support for the roles landmarks may play based on their size and location in way-finding and spatial knowledge acquisition. Based on the definition of global landmarks, their role is to serve as reference points and provide information that helps to structure the space. The roles of local landmarks are to 
provide information regarding decisions to be made while traversing routes and are argued to be associated with route knowledge. I will expand on the current body of knowledge of the roles each play as well as attempt to isolate the impact each has on the acquisition of route and survey related spatial knowledge and further the impact of each across the amount of familiarity.

Steck and Mallot (2000) are the only researchers to date who directly and explicitly investigated the role of both global and local landmarks in VE navigation. In their research, local landmarks were placed at junctions (e.g., buildings, phone booths), and global landmarks (e.g., hilltop, television tower) placed along the periphery of the environment and were intended as compass information or a "global frame of reference". The learning phase consisted of learning a route between two local landmarks. In the first study, the test phase consisted of rotating the positions of the local and global landmarks by $90^{\circ}$ in opposite directions. In the second study, fog was used to reduce the visibility of the global landmarks in one condition, and twilight was used to reduce the visibility of the local landmarks in another condition. Results from both studies showed that some people relied mostly on global landmarks, some on local, and some relied on a combination of the two, indicating a "personal preference" for global-local use. A confound within this study was that participants who noticed that the landmarks were transposed in the first study were not included in the analysis which excluded almost $40 \%$ of the participants. Those who noticed the transformation may have been participants who rely heavily on one or the other type of landmark and therefore their removal could have skewed the results one way or the other. These findings (Steck \& 
Mallot, 2000) together with those from Ruddle and Perch (2004) suggest that the use of global landmarks may be inconsistent and limited particularly when other cues are available. However, researchers have not investigated their roles regarding the acquisition of spatial knowledge, the way they are integrated into cognitive maps, or if each provides a different type of information for way-finding and cognitive map formation.

Although research points to different roles of landmarks based on their size and location, research regarding how they may be acquired and integrated into cognitive maps across experience is lacking. Next, I review literature concerning how spatial knowledge is acquired. This body of literature provides insight into my research questions in relation to when each landmark type is acquired and how their roles may change across familiarity.

Research discussed provides evidence for the possible relation between global landmarks and survey knowledge on one hand, and local landmarks and route knowledge on the other. The reason why global and local landmarks may play different roles, and how this difference is related to the processing strategy is discussed in terms of perception and attention to these features. The following section reviews literature related to perception and attention, specifically the way features are attended to within a visual scene. This review offers a different perspective as to why different roles may exist for different landmark types, and what contextual factors may direct precedence for one or the other. 


\section{Global Precedence}

The type of information perceived and attended to in visual scenes is welldocumented within perception and attention research (Hopkins \& Washburn, 2002; Kimchi, 1992; Miller, 1981; Miller \& Navon, 2002; Navon, 1977, 1983; Paquet \& Merikle, 1988; Pomerantz, 1983). This body of literature offers implications as to what features are visually attended to within a $3 \mathrm{D} \mathrm{VE}$, and the strategy used to acquire and recall information about a visual scene. In general, the literature provides two theoretical focal points for this dissertation. First, in terms of processing strategies used for acquiring spatial knowledge, research suggests that a similar strategy of information processing may be applied to processing global and local landmarks. Second, the visual attention literature implies that precedence for features change depending on certain contextual factors, such as duration of exposure, instructions provided, and size and type of stimulus being attended to. Extending this to landmark size and location within a 3D environment, this precedence could be dependent on certain contextual factors such as familiarity or aids provided. I therefore propose that the role global and local landmarks play within spatial knowledge acquisition and representation may be explained in terms of certain principles of perception and attention.

Attention to certain features within a visual stimuli is claimed to imply the processing strategy used. In a classic visual attention study, Navon (1977) hypothesized that the global structure of a visual scene will precede analysis of any local features, rather than perceiving all features at once. In Navon's words, “a scene is decomposed rather than built up..." (p. 354). The typical stimulus used within this research is referred 
to as a compound letter (see Figure 3), which affords the manipulation of both its global and local features. The stimuli's global feature would be the 'larger' global element, that is, the large H, whereas its' local feature would be the small letters that make up the stimulus.

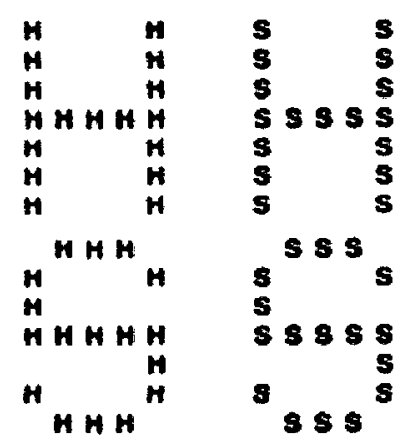

Figure 3. An example of Navon's (1977) compound letter stimuli.

In Navon's (1977) experiment, participants were asked to identify the large letter and to ignore the small ones (referred to as the global task), or identify the small letters while ignoring the large letters (local task). The stimulus in Figure 3 was used to explore global precedence. The stimuli on the top left and bottom right of Figure 3 are nonconflicting global and local features. The two stimuli on the left are referred to as "matching features", that is the global and local features match. Whereas the stimuli on the right are "conflicting features", that is the global and local features do not match.

The speed and accuracy for identifying the letters in each task were measured. Navon (1977) found that global features were extracted earlier and more accurately than local ones. Using this type of stimuli he demonstrated the advantage of global features over local as a function of time. Additionally, when the global and local features did not match (conflicting stimuli; see right side of Figure 3) participants' accuracy and speed 
were only negatively affected when asked to identify the local features. Navon (1977) interpreted his findings as evidence for global precedence in visual attention. At this time it should be noted that I do not consider global and local landmarks as equivalent to global and local features within the visual scenes used within this particular body of literature. In the perception literature the global features are literally composed of the local ones; a compound stimulus cannot exist without both global and local features. Within the spatial cognition literature these two features can be argued to be two mutually exclusive features within an environment and any given environment may have one or the other. I do however propose that the type of information attended to and the way in which they are attended to may be similar. As Navon suggests, by attending to the global features first the compound stimuli are decomposed rather than built up. The same might be said for learning a 3D space, that is attending to the global feature first, when available, may provide evidence for a top-down type of processing with the environmental features being decomposed rather than built up by way of attending to local landmarks first.

In general, the global precedence hypothesis claims that the processing of a scene begins with attention to the global properties first followed by local as time progresses. That is, global properties of visual stimuli are processed first, followed by analysis of local properties. If we extend this finding to the visual processing of $3 \mathrm{D}$ environments, the smaller landmarks could be equated with the local features, whereas the global features could be equated with the more topological structure of the environment or global landmarks. The larger, global letter is considered a higher level unit in relation to 
the smaller, local letters, which are the lower level units. Properties of the higher level unit are considered to be more global than properties of the lower level units by virtue of their position in the hierarchy. According to Pomerantz (1981) during the development of the percept, the global properties have temporal precedence. The development of a cognitive map can be thought of in the same way; however, it is still unknown if there is temporal precedence for certain landmark features within the microgenesis of spatial knowledge acquisition.

It should be noted that only static $2 \mathrm{D}$ displays, such as pictures and compound letter stimuli have been investigated within global precedence research. I can therefore only speculate as to what type of information is attended to sooner within a $2 \mathrm{D}$ map or 3D environment when it is confounded with other dynamic tasks, such as way-finding. Although most hypotheses and findings in this research rely on static images as its test bed, I argue that what is attended to in static visual scenes could perceivably transfer to 3D VEs. For example, if global landmarks provide information that helps to organize and structure the world, they may be attended to faster than local landmarks.

Interestingly, a large amount of spatial cognition uses 2D stimuli (such as slide shows or pictures), which can be compared in a more direct way to global precedence research (Cohen \& Schuepfer, 1980; Cornell \& Hay, 1984; Garling, Book, \& Lindberg, 1984). Therefore, it could be argued that the pervasiveness of global precedence may also occur for processing 3D representations (MacEachren, 1995). This perception and attention research is closely related to the way spatial knowledge is acquired as both paradigms investigate visual attention as a function of what type of information is 
extracted and when. Global precedence researchers claim that global features are attended to first when processing a scene or stimulus because we are extracting the meaning and then the details, indicating a hierarchical processing strategy. If there is evidence for global landmark precedence, then a hierarchical spatial processing strategy is also feasible. Further, this precedence could potentially be based on the location of landmarks. The second theoretical focal point of this visual attention literature is support for precedence of features that depend on contextual factors, such as time of exposure, and the size and type of attended stimuli. The size of the stimuli being attended to has been found to affect precedence for global features. For example, Antes and Mann (1984) were interested in investigating whether global precedence would transfer to scenes where these features have a relationship with one another. Their stimuli consisted of one of two scenes, a farm scene or a beach scene. The farm scene contained local elements, such as a tractor and barn that made up the global feature (i.e. a farm). Antes and Mann's (1984) participants were required to respond to the global features (farm or beach) or the local features (tractor and boat) within pictures. Global precedence was indeed found, but only when the pictures were small (4 inches) whereas local precedence was found for large scenes (16 inches). It could be argued that the large scenes would be most similar in attending to features within a real 3D environment leading to the claim that it could therefore be that local landmarks may have precedence when the stimulus is a real or virtual 3D VE.

Further research has found that global precedence changes depending on the object or scene being perceived, indicating that global precedence may not be as 
pervasive as Navon (1977) once argued. After years of research, certain factors have been found to play a role in whether the global or local features have temporal precedence in visual perception. Some examples include the size and type of stimuli, as well as the duration of exposure. In a further study Navon (1983) found evidence of local precedence when the local features of compound stimuli were more widely spaced. Duration of exposure to the stimulus is also argued to affect precedence. Paquet and Merikle (1988) found global precedence at very short exposures: $10 \mathrm{~ms}$, in compound letter stimuli, but not at $40 \mathrm{~ms}$. Duration of exposure, or the amount of familiarity with the environment, may be an important factor that affects attention and acquisition to global and local landmarks within real or VE's. For example, it is known that after short durations people are better at recalling structural landmarks such as doorways compared to object landmarks such as a plant in the hallway within a building (Stankiewicz \& Kalia, 2007).

A longitudinal study of cognitive maps and spatial knowledge acquisition conducted by Evans et al. (1981) found that college students' knowledge of landmarks on first arrival did not change when compared to their knowledge a year later. However the knowledge of paths off critical anchor points did improve over a longer duration of exposure. Further, (Holscher, Buchner, Meilinger, \& Strube, 2009) found that the more unfamiliar a building layout is, the more frequently participants looked towards the outside for landmark information that would help them orient themselves within the building. These examples provide initial insight as to how familiarity or other contextual factors could in fact influence precedence for one or the other. The impact that the duration of exposure to the virtual environment as a possible contextual factor that 
influences the attention to global and local landmarks will be explored as part of the current research plan.

One confound called to attention by this body of literature is whether it is simply a question of attending to the larger feature. That is, the larger the feature, the more priority it has in attracting attention. A second confound is that the majority of research has investigated static object-based 2D displays, using uncomplicated stimuli.

Regardless, the messages taken from this body of literature are important. Duration of exposure along with other contextual factors may play a role in global and local landmark attention and acquisition, and point to the way in which they may be integrated into the cognitive map. Therefore, upon review and synthesis of global precedence research it can be hypothesized that in way-finding, global landmarks may be attended to and learned first, followed by local ones, or vice versa depending on specific contextual factors at play such as their location.

Findings from spatial cognition and global precedence research will help to explain and understand the potential roles that global and local landmarks may play in acquiring spatial knowledge. For example, if global landmarks are associated with survey knowledge and local landmarks with route, the way they are attended to and acquired could provide insight into their role in way-finding and cognitive maps formation. Overall, global precedence suggests that when perceiving visual stimuli containing global and local features, the global properties are generally attended to faster. However, findings also reveal that precedence is not pervasive; it is dependent on contextual 
factors, which may also play a role in attending to and acquiring global and local landmarks while way-finding.

The processing strategy used may affect the way cognitive maps are constructed. The explanation brought forth by global precedence research is that different types of features may be associated with different types of processing. Either the global features are attended to first and the scene is then decomposed, or the local features are attended to first and then the scene is built up. As discussed previously, one view of how cognitive maps are formed is based on a linear, progressive strategy (LRS model) where the details are learned first, and through experience the features that are included within survey knowledge are then acquired (Siegel \& White, 1975). It can be suggested that local landmarks will play a more significant role in the early stages of constructing a cognitive map if this type of detail is attended to first and the global landmarks are ignored until later (Parush \& Berman, 2004). The second strategy is to attend to the more global features first followed by the details that are placed within this structure, provided by the global landmarks ((Jacobs \& Schenk, 2003); Parush \& Berman, 2004).

Overall, how we visually attend to features within various types of stimuli provides further explanation as to the different roles that the size and location of landmarks may play based on how information is processed. Not to say that the same cognitive processes are at play when comparing global and local features to global and local landmarks; however, they may prove to share similar cognitive processes in terms of how people visually attend to properties within their environments. Therefore, a theoretical focal point of this research emerges following a review of global precedence 
research in that there seems to be some support for a similar information processing strategy used to attend to features within a visual scene and for 3D environments.

Following a comprehensive review and analysis of related literature it is clear that the distinction between global and local landmarks is rooted differently: neurally, developmentally, and behaviorally, humans and animals respond to landmarks during way-finding; however the characteristics that make them so is largely unknown. For example, the findings could be based on their size or their location, or some combination of both. Further, the roles of each landmark type are not conclusively empirically supported. Figure 4 provides an overview of the current dissertations' research space and hypotheses. This figure provides a summary of each landmark's hypothesized role for spatial knowledge and processing strategy by its type and location.

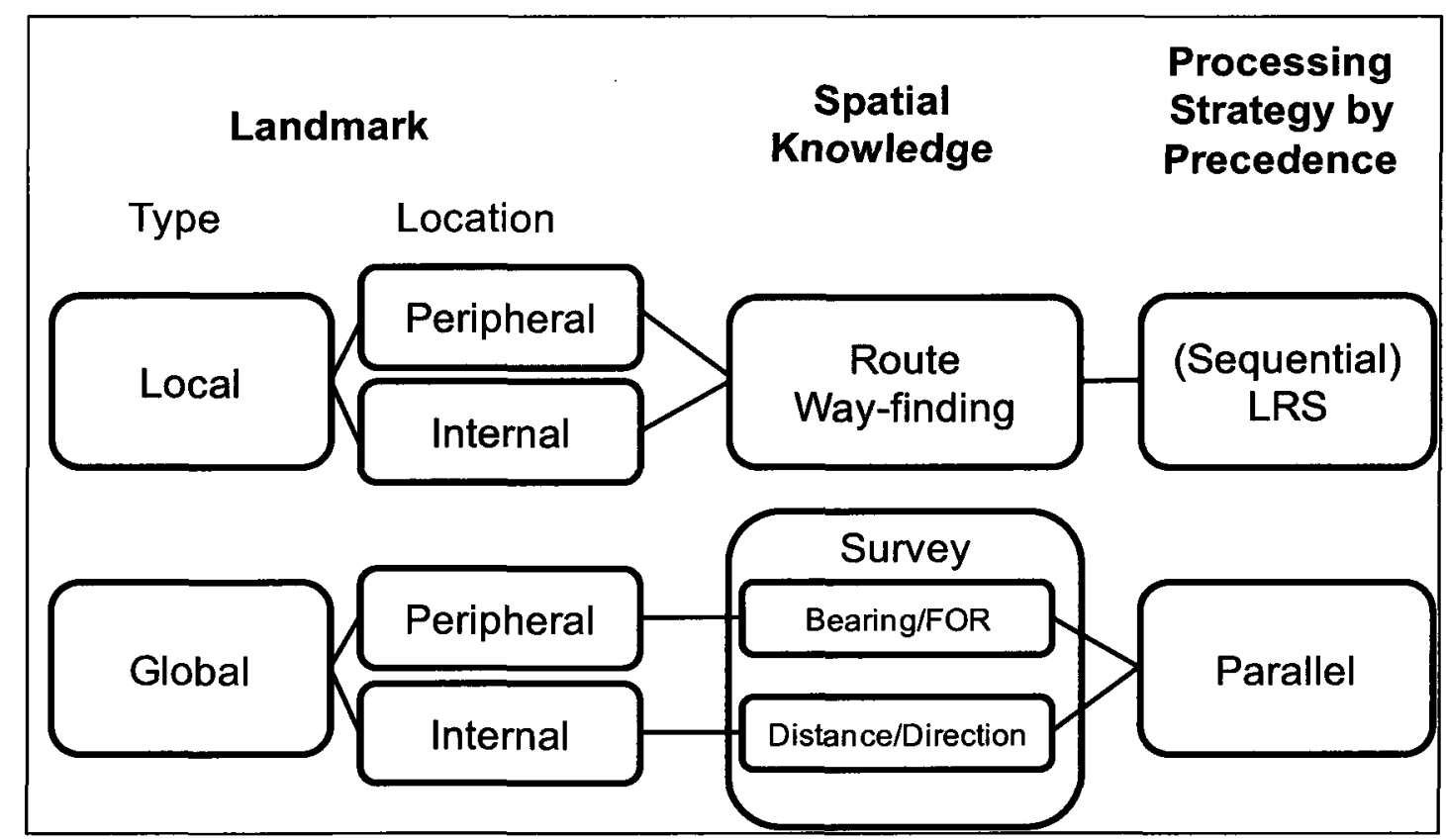

Figure 4. An overview of the research space and hypotheses for the current dissertation. 
Again, roles of local landmarks are hypothesized and found to be associated with route knowledge, and global landmarks are hypothesized to be associated with survey -and to a lesser degree perhaps route knowledge as well- given that they provide distance and direction information for way-finders. Therefore, the primary goal of this research program is to investigate the roles that each type of landmark may play based on its size and location within way-finding and spatial knowledge acquisition.

\section{Hypotheses and Research Paradigm}

This dissertation aims to examine the role of global and local landmarks in wayfinding and spatial knowledge acquisition. It should be noted that there is no prior research that has investigated global and local landmarks while controlling for their locations, making these hypotheses speculative. Based on initial review, critique, and synthesis of the literature I hypothesize that the type of information that landmarks provide for acquiring and completing route and survey knowledge based tasks will depend on a combination of their size and location. More specifically, large landmarks located in the periphery of the world are hypothesized to provide a frame of reference or bearing information, whereas the large landmarks located inside the environment are hypothesized to provide distance and direction information, but less related to the provision of a frame of reference when compared to those located in the periphery. As local-internal landmarks are visible only from a short distance, placed at decision points and in turn relied upon to make route decisions while way-finding, it is hypothesized that those located in the periphery will exhibit identical roles as the "size" of the landmark is the characteristic hypothesized to define its role to a greater degree than its location. That 
is, roles associated with local landmarks will be independent of their location, categorized as peripheral versus internal.

This dissertation aims to examine the roles of two characteristics of landmarks in wayfinding and spatial knowledge acquisition, their size and location. The processing strategy and factors that may control attention and use to each type at each location will be explored throughout the proposed research plan. To eliminate the ambiguities of landmark features the design of each landmark within the world was classified based on their size, global or local, and their location, peripheral or internal. All other objects in the world could not be used as landmarks as their features were identical. In order to "force" the use of these objects as landmarks no other navigation aids were present. The research plan and approach involved revealing the impact global and local landmarks have within 3D desktop VEs on way-finding performance and spatial knowledge acquisition by way of three experiments. Figure 5 presents a visual overview of the three studies used to investigate the roles of landmarks by their type and location. Experiment 1 investigated the roles played by the size of landmarks in isolation. Experiment 2 investigated the roles played by the location of landmarks, in isolation and Experiment 3 investigated the roles played by the combination of size and location, across familiarity to gain insight into how they are attended to, acquired, and integrated into our cognitive maps. 


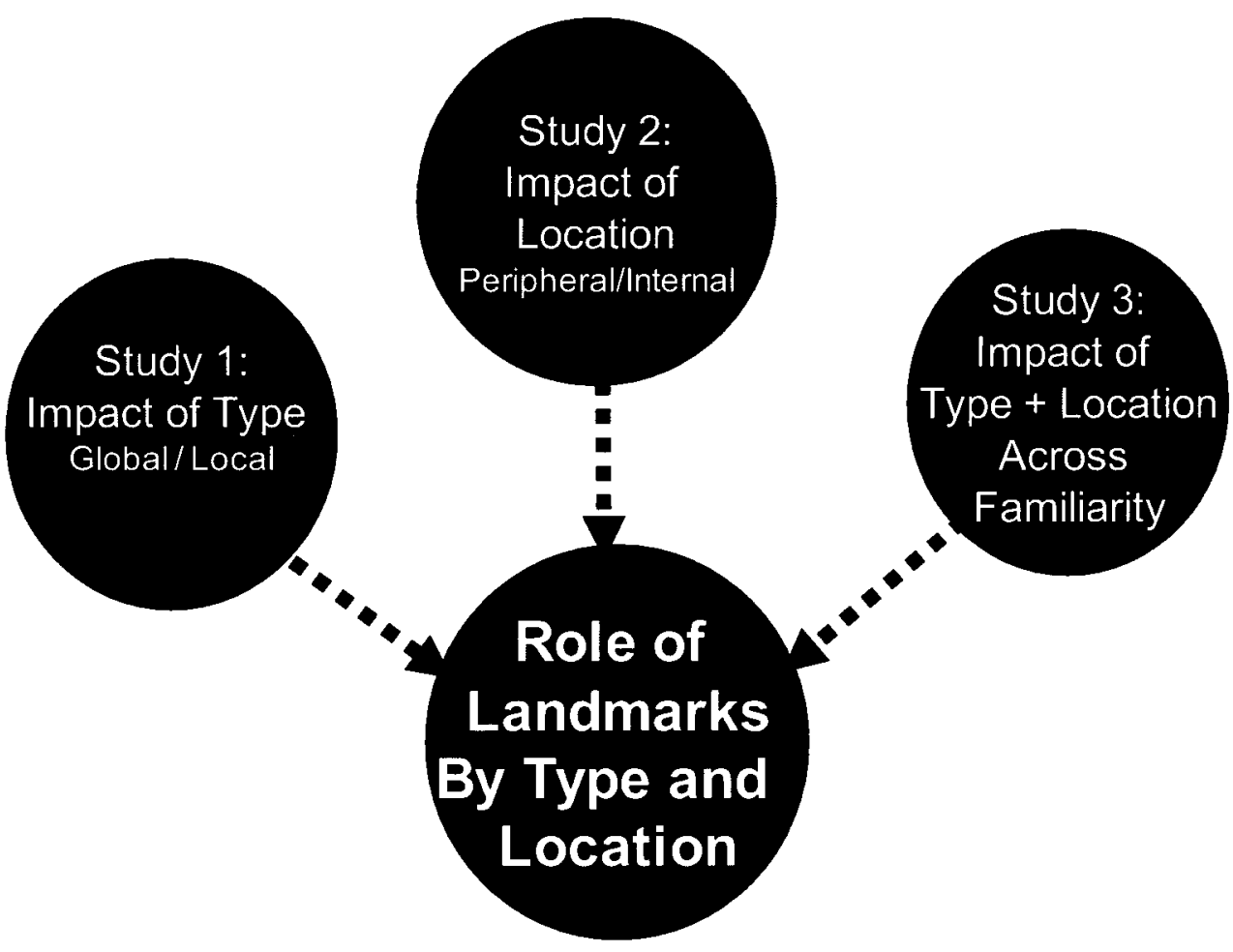

Figure 5. An overview of the three experiments for the current dissertation. 
Experiment 1: Isolating the Impact of Landmark Type

As discussed in the general introduction, studies have demonstrated that the absence of landmarks has a negative impact on way-finding and spatial knowledge acquisition. However, very little research has isolated and defined the impact of different types of landmarks. As such, the nature of information provided by global and local landmarks and whether the presence of only one type can still aid people as they find their way and acquire spatial knowledge is not clear. This first experiment attempted to isolate the impact of global and local landmarks and reveal the roles each plays in wayfinding and spatial knowledge acquisition. The presence of landmark type, global or local, was independent of location. That is, global and local landmarks were present in the periphery and the internal part of the world. The following questions were investigated in Experiment 1:

Are both types of landmarks (global and local) equally essential for way-finding and spatial knowledge acquisition?

a. How will the absence of one type effect way-finding performance?

b. How will the absence of one type effect the acquisition of spatial knowledge?

c. Within each type of landmark (global and local), will its location play a role in their acquisition and use?

Three conditions were investigated; one group was provided with global landmarks only, a second with only local and a third was provided with both types, acting as a control group. The control group was chosen to experience both types of 
landmarks, rather than none, for two reasons. First, previous research has provided significant evidence that way-finding is difficult in environments without landmarks. Therefore a control group that was provided with neither type of landmark would not have provided any further theoretical benefit. Second, it was imperative to understand what significant benefit, if any, the presence of both global and local landmarks has compared to the presence of only one type independently to investigate the research questions posed.

Based on the literature reviewed and discussed in the general introduction, It was hypothesized that global landmarks may provide information that mediates the acquisition of survey knowledge and may help to structure the environment. If this hypothesis is supported, groups exposed to global landmarks will perform better on the map drawing survey knowledge task, compared to those exposed only to local landmarks. I also hypothesized that local landmarks would provide information that mediates the acquisition of route knowledge, which would aid in way-finding decision making, and confirmation that one is on the correct route, regardless of their location. If these hypotheses are supported, groups that were exposed to local landmarks should perform better on way-finding related tasks. Finally, I was hypothesized to find precedence for global landmarks. This hypothesis is supported by the claims made by researchers (Couclelis, et al., 1987); Lynch, 1960) and others that while in an unfamiliar environment we attend to global landmarks to orient first, followed by attention to local in order to way-find. This was investigated via way-finding performance. Finally, I hypothesized that landmark location will only have an effect on spatial knowledge and 
way-finding performance for global landmarks, but not local. The hypothesis is based on the notion that global landmarks located in the periphery provide more beneficial information (e.g., a frame of reference) to way-finders than global landmarks located internally, which perhaps will provide only direction or distance information. Finally, it was hypothesized local landmarks, as they cannot be seen from a distance, not to differ with regards to the information provided by their peripheral or internal locations.

\section{Method}

\section{Participants}

Sixty undergraduate students (30 males, 30 females) were recruited via Carleton University's online psychology participant recruiting website and assigned to one of the three groups $(\mathrm{n}=20)$. Although gender is known to have an impact on spatial ability to include it as a variable of interest was outside the scope of this thesis. Therefore gender was controlled for by ensuring an equal number of males and females in each group. Participants were given course credit for participation, which took approximately 1.5 hours. The mean age of the population tested was 21 years $(\mathrm{SD}=5.15)$. Apparatus and Materials

Three first-person desktop virtual worlds -one for each experimental conditionwere built using a series of tools to create, compile, and render objects. These tools are summarized in Table 3 . The world simulated a small town with streets laid out in a $6 \times 6$ rectangular grid with all sides surrounded by water. The configuration was identical for all worlds used in my dissertation (see Figure 6) and differed only in the landmarks placed throughout the world. This particular screen shot is of the world that includes both 
global and local landmarks, experience by the Both group. The size of the world was 180 units $\mathrm{x} 130$ units and the speed of travel was kept at a constant 5 units per second. A firstperson perspective screen shot can be found in Figure 7. This particular screen shot is of the world that includes both global and local landmarks, experienced by the Both group.

Table 3

Tools Used to Create, Compile and Render the VEs

\begin{tabular}{ll}
\hline \multicolumn{1}{c}{ Tool } & \multicolumn{1}{c}{ Use } \\
\hline Wally & Compile images (.bmp) into textures (.wad) \\
Valve Hammer Map Editor V3.4 & Create the world and objects (.map) \\
Script (builder.exe) & Script used to compile and render the world from \\
& a .map to a .wrl file \\
Script (Engine.exe) & A script used to run the world \\
\hline
\end{tabular}




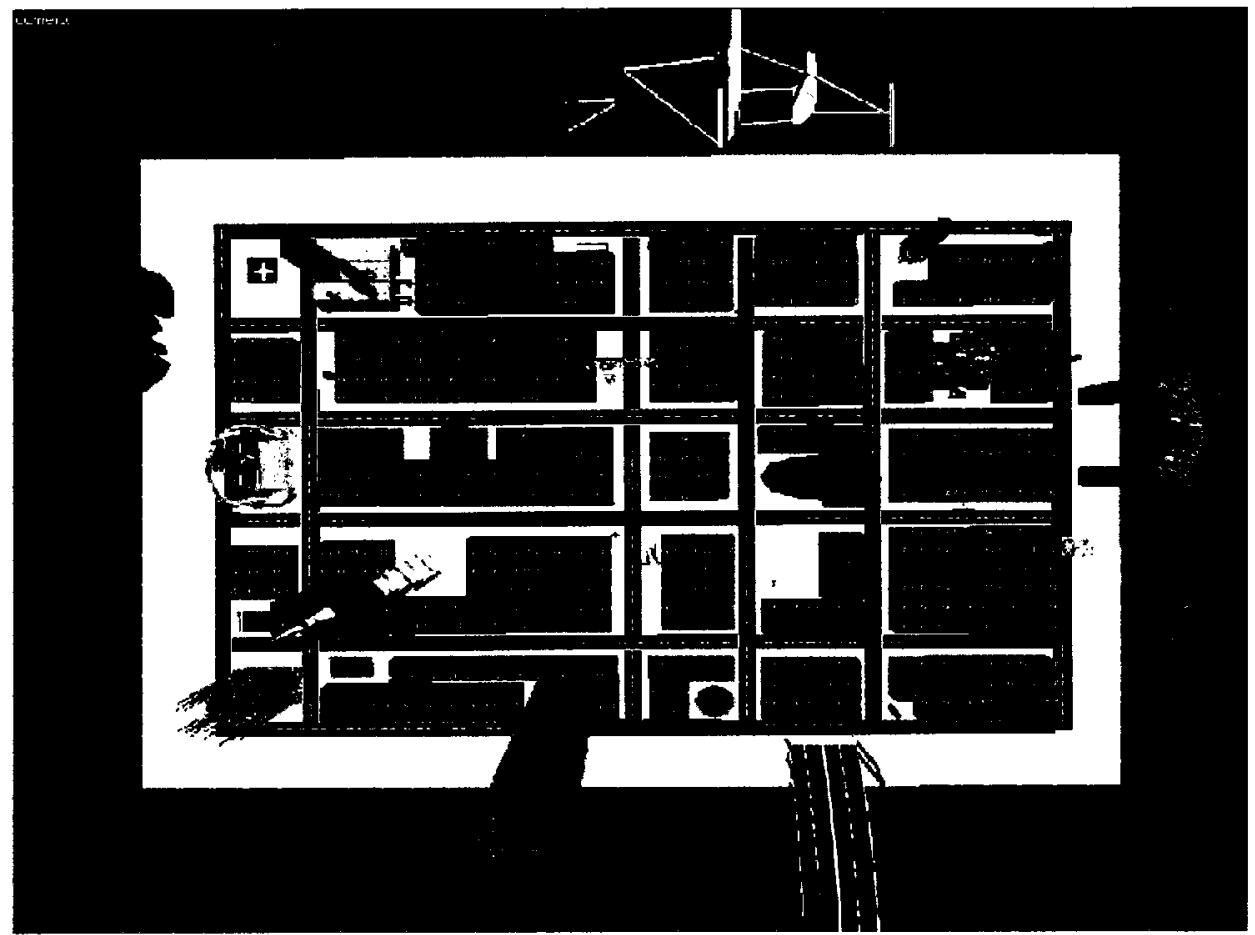

Figure 6. Birds-eye-view of the VE.

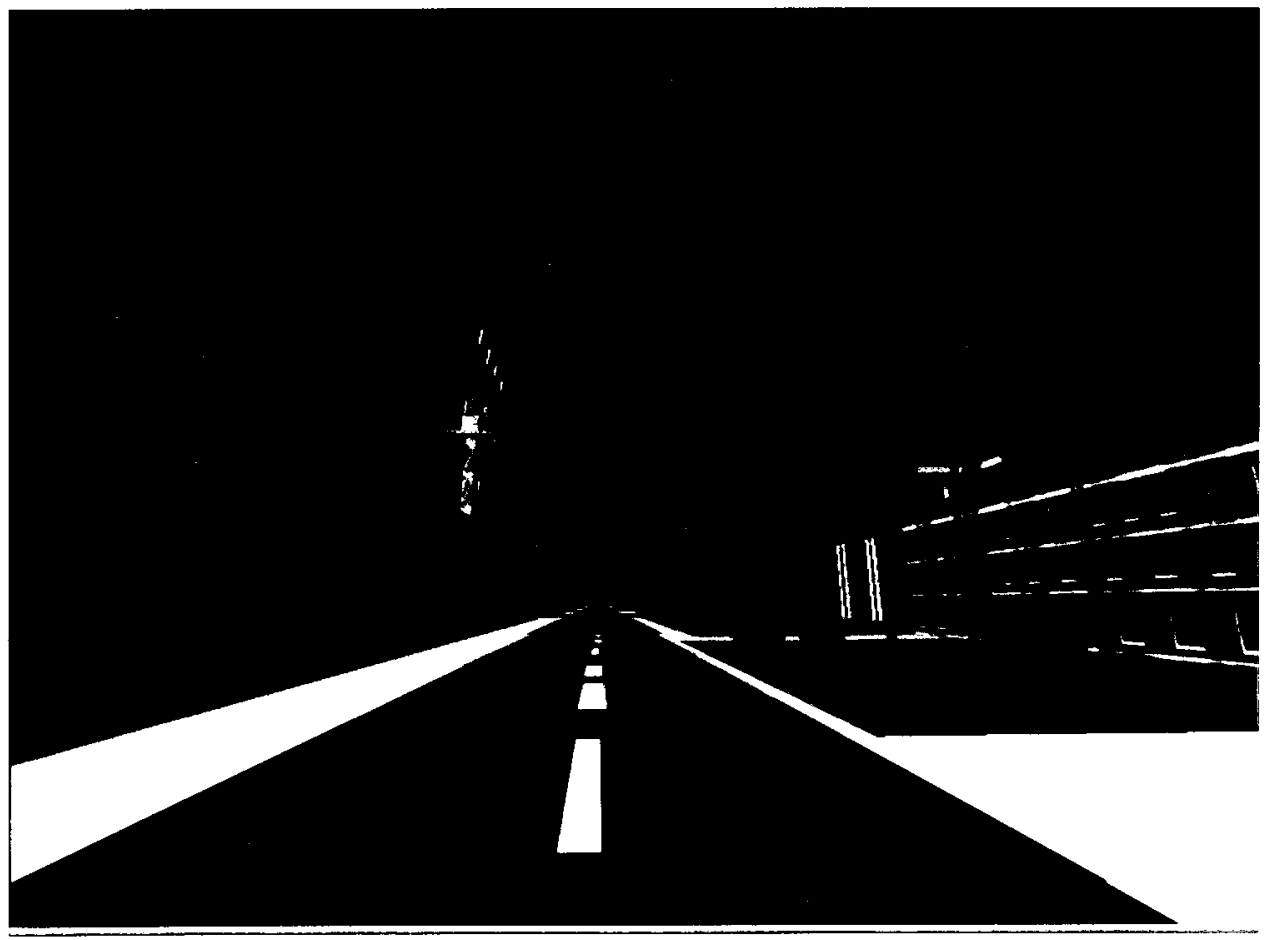

Figure 7. First-person view of the VE. 
Landmark design. As no explicit guidelines exist as to how to design global and local landmarks, the most concrete guideline was that global landmarks could be seen from most places in the environment and local only from short distances. Therefore, global landmarks were designed to be seen from most places within the environment, as defined in the literature, whereas local landmarks were designed to be seen from a short distance. A quantitative analysis of the visibility between global and local landmarks was completed and can be found in Appendix A. On average the global landmarks ( $\mathrm{M}=$ 18.11) were seen from more places within the VE than local $(M=5.33)$. Peripheral landmarks were categorized as those located on, or beyond, the outer-most road, and all others were categorized as internal landmarks. Landmarks were chosen to be as "ecologically" valid as possible. For example, a ship would was found in the surrounding water as would be expected, not in the middle of the city; a street light was found in an intersection, not in the water and so forth. It is possible that personal relevance of specific landmarks used to navigate may increase recall of specific landmarks. Therefore each landmark was chosen to be generic with the goal of ensuring that each landmark has the same personal relevance to each participant. A table outlining all landmarks, their type, and pictures can be found in Appendix B. A description of each VE created and used in Experiment 1's paradigm can be found in Table 4. 
Table 4

A Description of VE's used in Experiment 1

Group

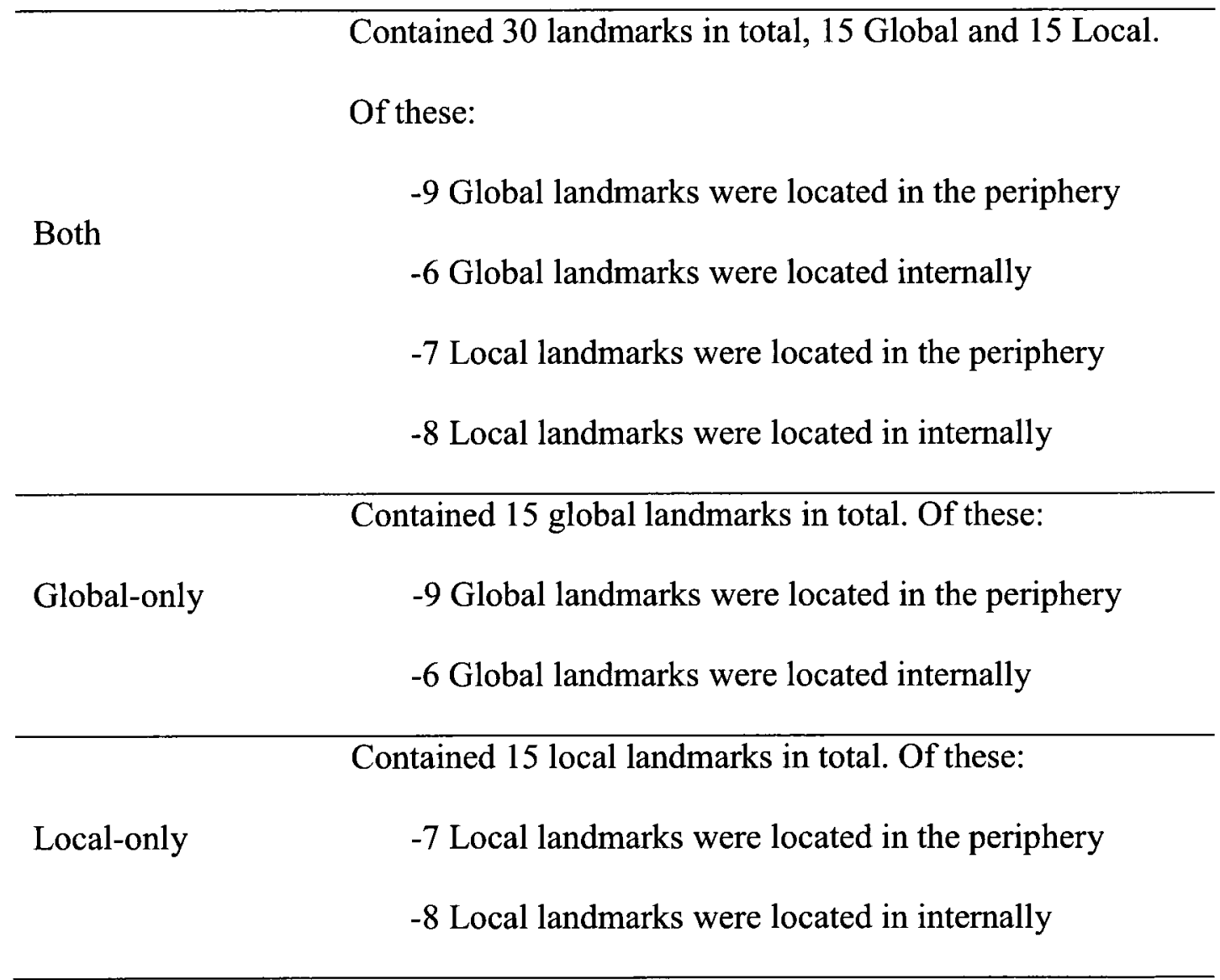

All stimuli created for the experimental tasks, as well as any instructions presented were created using a combination of MS Power Point and Paint programs. The E-prime software package, version 1.1 (Psychology Software Tools) was used to program, randomize, run, and record responses. The virtual environment and the experimental questions were presented on a 17 -inch monitor, set to a resolution and 24 bit colour depth. This computer had a Pentium IV processor and ran on Windows XP. 
Paper and pencil were also used to administer all questionnaires, and other experimental tasks.

It is well known that large individual and gender differences exist across spatial abilities (Blajenkova, Motes, \& Kozhevnikov, 2005; Hegarty, Montello, Richardson, Ishikawa, \& Lovelace, 2006; Peters et al., 1995). Therefore, the Revised Vandenberg Mental Rotation Test (MRT-A), a redrawn version of the original Vandenberg \& Kuse MRT was used as a potential covariate in order to control for gender differences of spatial abilities that may exist in the tested population (Peters, 2005; Peters, 1995). In the MRT-A, participants viewed a three-dimensional object and four test objects. Their task was to determine, as quickly and accurately as possible, which of the two test objects were rotations of the target object. This test consisted of two sections with 12 questions in each, for which participants were allotted a total of eight minutes, four for each section. See Appendix $\mathrm{C}$ for an example of questions taken from the MRT-A revised Vandenberg Mental Rotation Test (Peters, et al., 1995; Vandenberg \& Kuse, 1978).

Study Design

A between-participant design was employed with participants randomly assigned to one of three conditions:

1. Both group: received the Both virtual environment that contained 15 global and 15 local landmarks,

2. Global-only group: received the Global-only virtual environment that contained 15 global landmarks, and; 
3. Local-only group: received the Local-only virtual environment that contained 15 local landmarks.

Each group experienced two experimental phases:

1. Phase 1 - Way-finding and target search consisting of 15 target search trials.

2. Phase 2 - Spatial memory tasks consisting of four tasks: map drawing, judgment of relative direction (JRD), relative distance estimation (RDE), and a way-finding decision task (WDT). Although all four tasks were administered and analyzed, only one survey knowledge task; the map drawing, and one route related task; the WDT, were included in the results section. The included tasks are referred to as the 'primary tasks' whereas the $\mathrm{JRD}$ and RDE analyses are referred to as the 'secondary' tasks. The two secondary tasks did not provide any significant contribution to this dissertation and were therefore not included in the body of the results. The lack of consistent results across variables and experiments for the secondary tasks in turn made it quite difficult to compare across experiments and groups with any empirical vigor. The questions, procedure and results associated with the JRD and RDE tasks can be found in Appendix E. Figure 8 provides an overview of the design used in Experiment 1. 


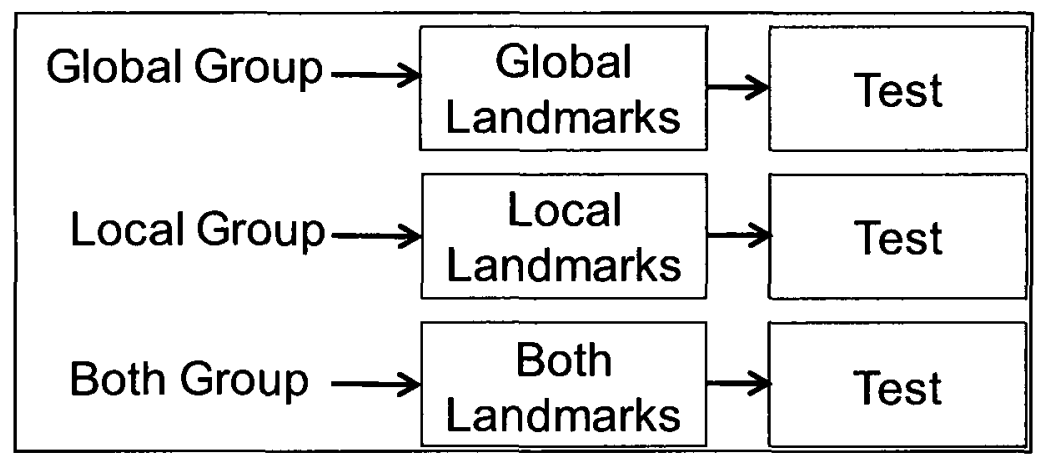

Figure 8. An overview of Experiment 1's study design.

\section{Procedure}

In a quiet room, participants individually gave informed consent (see Appendix F) and completed the following tasks:

1. Demographics Questionnaire. Participants answered questions regarding their age, gender, education, health, and gaming experience (see Appendix F.)

2. Revised Vandenberg Mental Rotation Test (see Appendix C for an example).

3. Cybersickness Questionnaire. With VE experiments, there is always a concern about the possibility of cybersickness (also referred to as VE sickness, simulator sickness). Cybersickness has three major components: nausea, oculomotor discomfort, and disorientation (Cobb et al., 1999; Kolasinksi, 1996). There are substantial differences in the extent to which different display technologies elicit cybersickness and substantial individual differences in susceptibility to cybersickness (Cobb et al., 1999). In all three studies described in this dissertation, the VE will be displayed on a desktop computer screen of the type commonly found on office workstations. Desktop displays induce the least 
amount of cybersickness (Cobb et al., 1999) because they incorporate the fewest causative factors. Nonetheless the following preventative measures were taken to avoid participants obtaining cybersickness:

a. Participants who were not in good health were not allowed to participate as ill health increases susceptibility to cybersickness (Kolasinski et al., 1995). Recruitment procedures specified that only subjects in good health can participate.

b. Participants were required to take breaks during the course of the experiment.

c. A cybersickness questionnaire was administered prior to and following any interaction with the VE (see Appendix D). If participants displayed or verbalized any sign of sickness they were asked not to continue with the experiment and their data was not used as part of the current studies.

Although no specific numbers were kept it is estimated that approximately $20 \%$ of females displayed or verbalized signs of cybersickness and consequently were asked to discontinue with the study.

4. Way-finding: Participants were presented with instructions regarding how to navigate the space and collect the targets (see Appendix $\mathrm{H}$ ) and was followed by a 5 minute practice trial began to allow participants to become familiar with navigating through a different but similar VE. Participants were then required to locate 15 targets that consisted of a floating cube with a numeric identifier, in a random order without replacement. Participants had 5 minutes to find each target 
during the testing phase. The time constraint was based on two considerations. The findings from a pilot study suggested that most targets were found within 5 minutes; and if a participant did not locate any targets, their maximum time spent locating targets would be controlled for ( 15 targets $* 5$ minutes $=75$ minutes $)$. After completing each trial, successfully or not, participants proceeded to the next trial. Measures were taken to ensure that the distance and number of turns between each starting location and target was equivalent across all 15 targets. For a full list of $\mathrm{x}, \mathrm{y}$ coordinates for each target location and its associated starting position, distance between targets and starting position, orientation angles, and number of turns for each target are outlined in Appendix I.

5. Spatial Memory Tasks: Participants were given two primary spatial memory tests to assess the acquisition and accuracy of spatial knowledge acquired during wayfinding. These two tasks are discussed in detail below.

a. Map Drawing: Participants were given ten minutes to draw, from memory, a map of the environment and were told to include and label all objects and features that they could remember. Measurements of participants' survey knowledge of the world included landmark knowledge, relative landmark location, and an overall score of how well the maps matched the actual VE. The use of sketch maps as a valid measure of internal cognitive maps of virtual environments has been supported by findings from Billinghurst \& Weghorst (1995). 
b. Way-finding Decision Task (WDT): A multiple choice task in which participants chose which way-finding action was to be taken next in order to reach the goal. Referring to the example below in Figure 9, participants were asked to decide which action would be required to reach the mountain. In the example shown the participant is asked in which direction to proceed to reach the mountain, a global landmark. The Both group received questions that referred to global and local landmarks. Global-only group received questions that referred to only global landmarks and Local-only group received questions that only referred to local landmarks. See Appendix $\mathbf{J}$ for the list of questions used in this spatial knowledge task. All 12 questions were randomly presented.

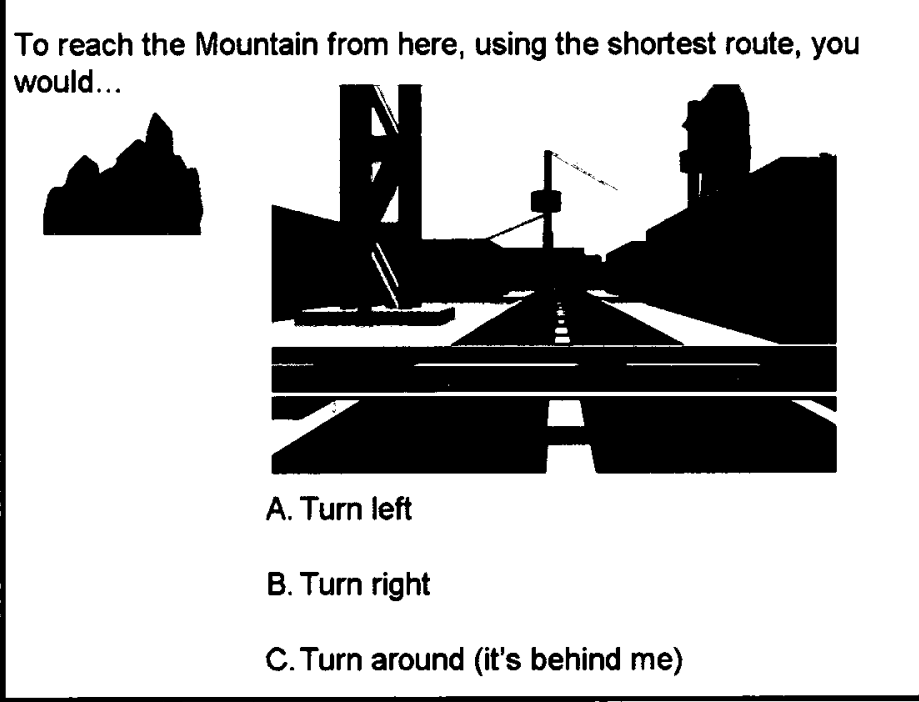

Figure 9. Way-finding Decision Task question.

c. Spatial strategy questionnaire: Participants answered questions regarding the strategies they used to way-find and acquire spatial information about 
the environment. The questionnaire, found in Appendix K, was an adapted version of the way-finding strategies scale produced by Lawton \& Kallai (2002). Lawton and Kallai (2002) examined individual differences in way-finding strategies. Participants were asked to report which strategy they prefer when navigating through the environment: orientation or route. Orientation strategies involve maintaining a sense of one's own position in relation to a given a point. An example of this type of question was "I keep track of the direction in terms of north, south, east, or west, in which I am going". Whereas route strategies involve using a particular route to get from place to place. An example of this type of question was "I ask for directions telling me whether to turn right or left at particular landmarks". Differences found between groups across the various orientation versus route related strategy questions provided insight into the different way-finding strategies possibly associated with the specific type of landmark available.

\section{Measurements}

1. MRT-A: The MRT-A score was calculated by giving one point per question if the participant matched both of the stimulus figures to the target figure correctly. No credit was given for a single correct answer, for a maximum score of 24 (Peters et al, 1995). Each analysis included a test to determine if spatial ability as measured by the MRT-A was an appropriate covariate to control for gender differences that may exist. 
2. Navigation duration: The mean time from the beginning of each trial until the target was found.

3. Number of targets found: The total number of targets found across 15 trials.

4. Sketch maps:

a. Map goodness scores: Map goodness was used to analyze the drawn maps. This technique was adapted from previous research (Gauthier et al., 2008; Stankiewicz \& Kalia, 2007). Maps were ranked for goodness on a scale of 1-5 by three judges who were familiar with the experimental environment but blind to the conditions. The map raters were provided with an overview with the world that they were rating but had no first-hand experience within the $3 \mathrm{D}$ desktop VE. The raters were told to rank each individual participants' map on how useful they would be as an aid to find their way around the world. They were also told to ignore the participant's drawing ability and to focus more on how well the map represents the world and the locations of the objects within it. Each map rater across all experiments were provided with the same instructions.Inter-rater reliability was calculated and the mean map goodness score was used as a measure of participants cognitive map formation.

b. Landmark inclusion: The total number of landmarks included in maps.

c. Correct landmark placement: The ratio between the numbers of correctly placed objects and the total number of objects drawn on the map.

5. WDT accuracy: The mean number of correct WDT answers. 


\section{Results}

It was hypothesized that, in isolation, global and local landmarks would have different effects on way-finding performance, route knowledge, and survey knowledge. Specifically, degradation was expected in survey knowledge with the absence of global landmarks, and degradation in route knowledge was expected in the absence of local landmarks. I also hypothesized that attention to, or use of, global landmarks will temporally precede local landmarks. Therefore it is hypothesized that the global-only group would outperform the local-only group during early way-finding performance. Given that the targets are found in both peripheral and internal locations it is hypothesized that no differences between landmark locations on way-finding performance for local landmarks would be found.

Participants' time to locate targets, number of targets found, landmark and landmark location recall and sketch map goodness ratings, WDT performance and spatial strategy questionnaire responses were analyzed. To examine and potentially control for confounding impact of spatial ability on performance, the MRT-A score was calculated. MRT-A was not used as a covariate as the scores did not differ significantly between groups and were not significantly correlated with any dependent variables.

All dependent variables were checked for outliers, missing data, normality, and homogeneity of variance and can be assumed to comply unless otherwise indicated. The assumption of homogeneity of covariance matrices was examined using Box's test of equality of variance matrices, and Levene's test examined for equality of variances. Most of the comparisons in this dissertation were made by way of planned comparisons that 
were driven by apriori hypotheses supported by the literature and current theses.

Analyses of Variance (ANOVA) were used when interactions were of particular interest or if comparisons were to be made between more than two groups. Any ANOVA post hoc comparisons were completed using Bonferonni correction for all alpha levels, however there was no correction made for planned comparisons that were based on a priori hypotheses. Standard errors bars are present on all figures to aid in interpretation and descriptive statistics are provided in associated appendices.

Way-finding and Target Search

Participants were required to locate 15 targets over a series of 15 trials. The 15 trials were divided into three blocks consisting of five trials each. It was hypothesized that landmark type would influence way-finding performance as a measure of the time taken to locate the targets across time (blocks) and the number of targets located in total. 'Time to find targets' was found to violate the assumption of normality. To deal with this violation, the variable of 'time to find targets' was transformed into its natural logarithm. This transformation was used across all three studies and provided data that was normal. Further once transformed the analyses proved to be more conservative compared to when the time to find targets was in it raw form. All descriptive statistics associated with wayfinding performance can be found in Appendix $\mathrm{L}$.

Planned comparisons were used to test the hypothesis of global or local precedence during early versus later way-finding. It was hypothesized that if attention, or use, of global landmarks precedes local landmarks, those exposed to only global landmarks will outperform those exposed to only local landmarks during way-finding. 
However, if local landmarks temporally precede, participants exposed only to local landmarks should perform better. These hypothesized were investigated by comparing group differences for early learning (Ln mean of first block) and later learning ( $\mathrm{Ln}$ mean of last block). Planned-comparison independent sample t-tests were used to test differences between groups at the first and last blocks respectively (see Figure 10). The Global-only group found targets in a significantly shorter amount of time in the first block, $t(38)=-2.267, p<.05$, when compared to the Local-only group. When the last block was analyzed for differences, the opposite finding was true with the Local-only group outperforming the Global-only group (see Figure 10). The Local-only group took less time to find the targets, $t(38)=1.797, p=.08$, relative to the Global-only group, however only marginally significant.

It was hypothesized that, regardless of group, participants would improve across time. Planned paired sample t-tests were used to test for performance improvement across time between the first and last block within each group. This analysis revealed that the local-only group significantly improved across time, $t(19)=8.051, p<.001$. However, the global-only group did not show significant improvement over time. 


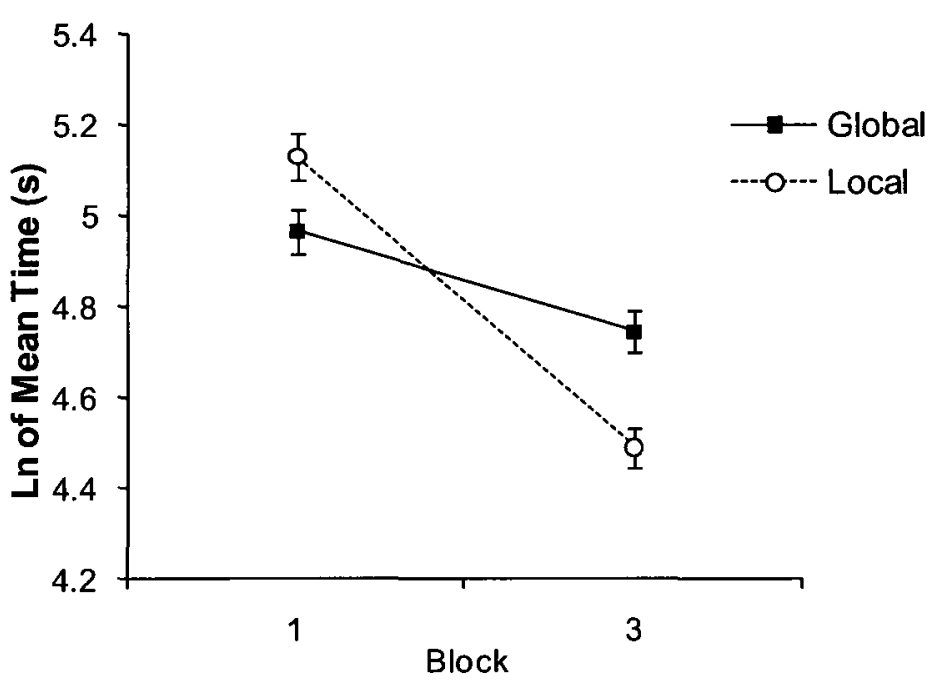

Figure 10. Mean of the Natural Log of time in seconds to locate targets (+SE) as a function of blocks for Global and Local-only groups.

A second analysis was conducted to investigate the number of targets found between groups. Local landmarks are hypothesized to aid in locating targets. Therefore it was expected that the local-only group would find more targets than the global-only group regardless of block tested. This analysis also provides insight into whether there was a trade-off between the number of targets found and time spent in the world. That is, although a group may have taken a longer time to find targets; they may have found more of them overall. Planned independent t-tests were used to test for differences between groups. The t-tests revealed that in the first block the global-only group found significantly more targets, when compared to the local-only group $t(37)=2.366, p<.05$. However during the last block this finding was reversed with the local-only group locating significantly more targets, $t(26.534)=-2.080, p<.05$ (see Figure 11 ). 
Planned paired sample t-tests were used to investigate improvement across time within each experimental group. This was measured by the difference between the first and last block. The local-only group found significantly more targets in the last block when compared to their first block, $t(19)=-5.877, p<.001$ (see Figure 11). However, the global-only group did not show significant improvement over time.

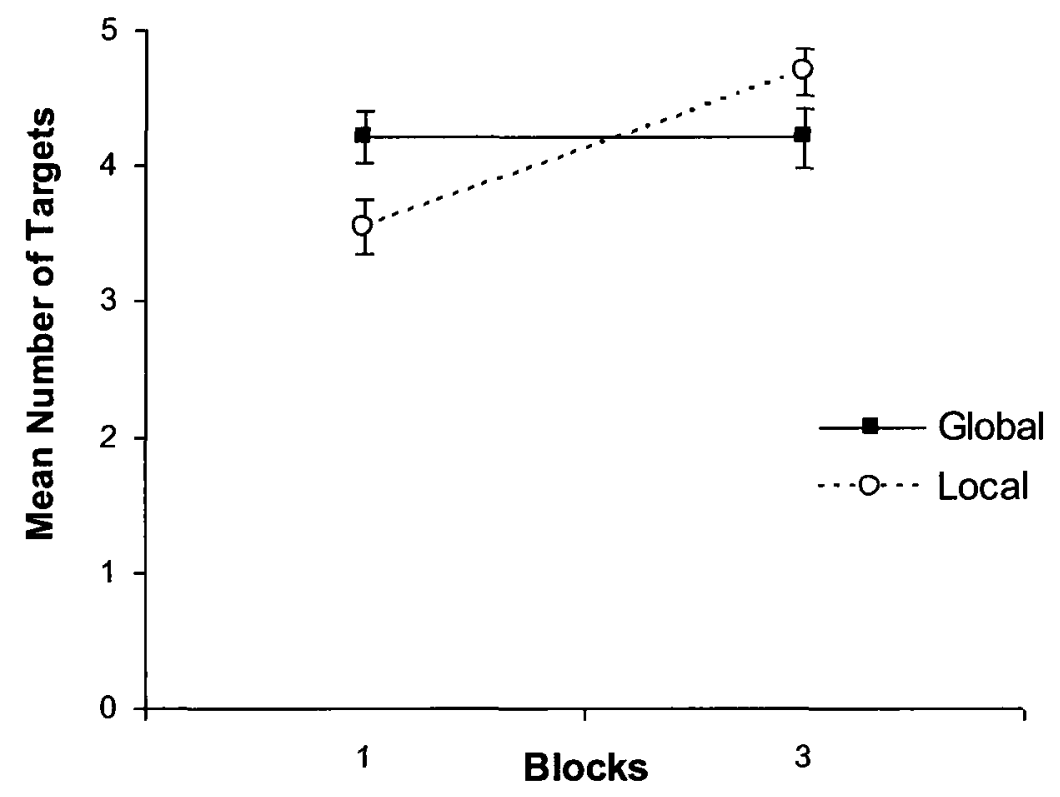

Figure 11. Mean number of targets located $(+\mathrm{SE})$ as a function of blocks for Global and Local-only groups.

Overall, two key findings can be extracted from the way-finding results. First, participants exposed to global landmarks performed better earlier during the way-finding process (i.e., block 1). However, as participants had more way-finding practice (i.e., block 3) the opposite was found; those exposed to local landmarks performed better. The second key finding was that although participants exposed only to global landmarks 
performed better during initial way-finding, this group showed no significant improvement over time, whereas those exposed only to local landmarks only showed a significant improvement over time.

Spatial Memory Tests

\section{Sketch Map Analysis}

Sketch maps are typically used to analyze the integrity of acquired landmark and survey related knowledge (Billinghurst \& Weghorst, 1995). Landmark knowledge includes two types of information: pure landmark recall (no associated spatial information) and the location of the landmark within the world. The following analyses investigated group differences of landmark knowledge, as measured by the number of landmarks included. In addition, the correct placement of the landmarks and overall map 'goodness' were analyzed to assess groups' survey knowledge. An example of a sketch map from each group is provided in Figure 12. All descriptive statistics associated with sketch map performance can be found in Appendix M. 
(a)
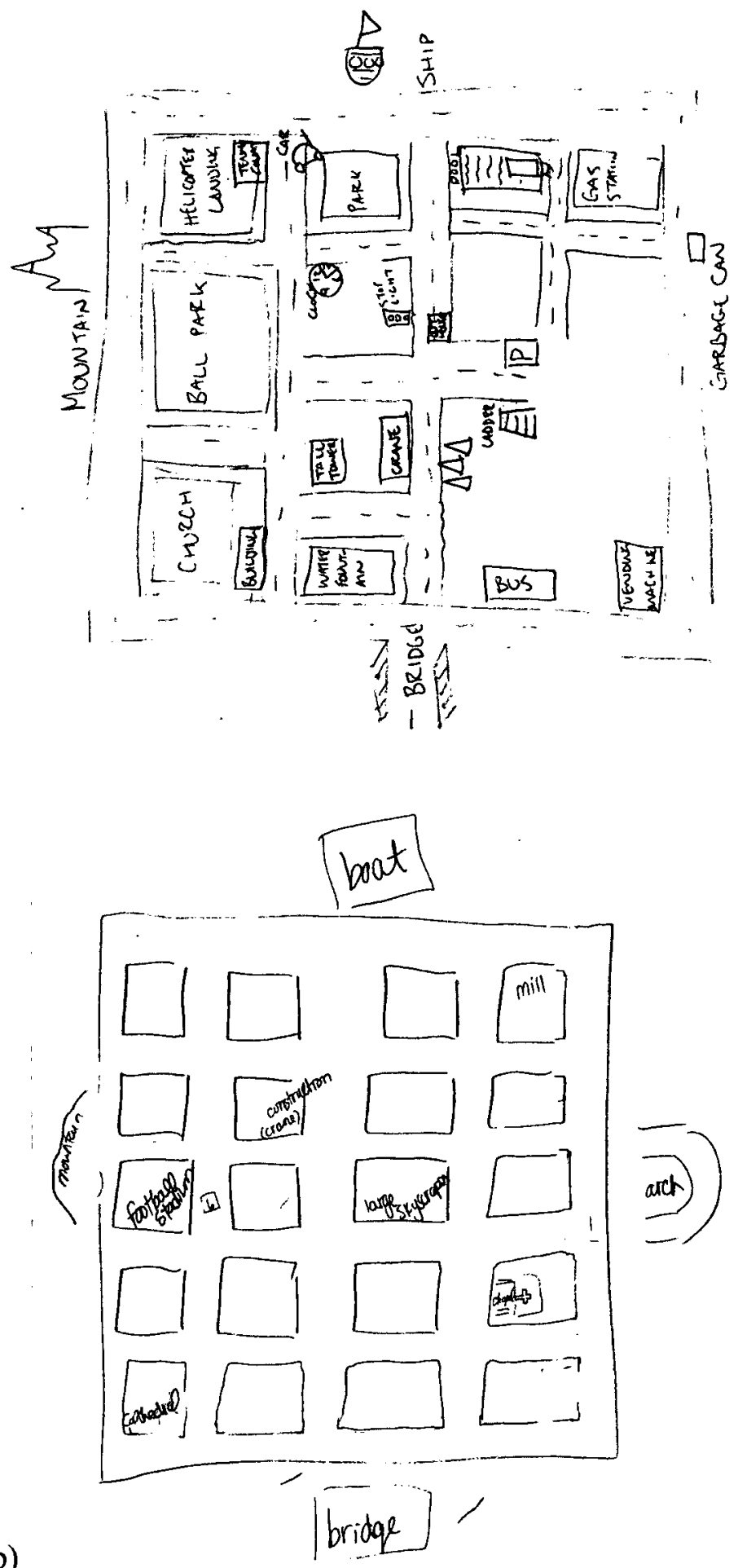

(b) 
(c)

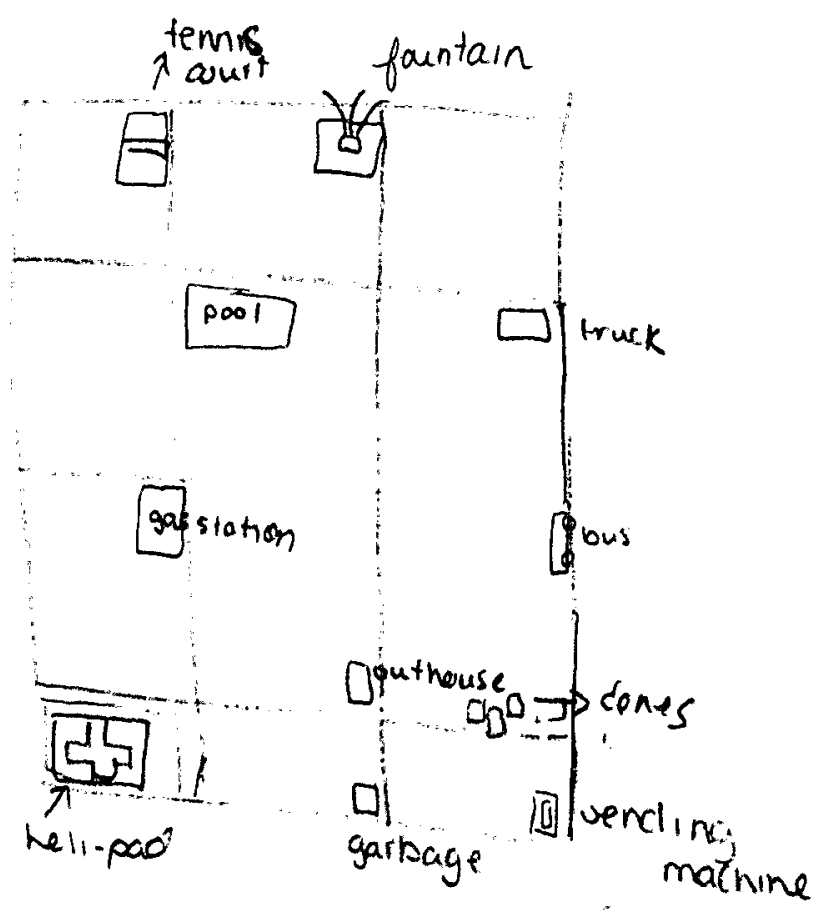

Figure 12. Sketch map examples from the (a) Both, (b) Global, and (c) Local-only groups.

Landmark knowledge by group. To investigate group differences of landmark knowledge the ratio between the numbers of landmarks placed on the sketch maps and the total number of landmark was computed. This is a revised score adapted from Billinghurst and Weghorst (1995). It was hypothesized that there would be no group differences between the Global and Local-only groups. A planned comparison independent t-test, used to investigate differences between mean number of landmarks for the Global and Local-only groups, did not reveal any group differences (see Figure 13). 


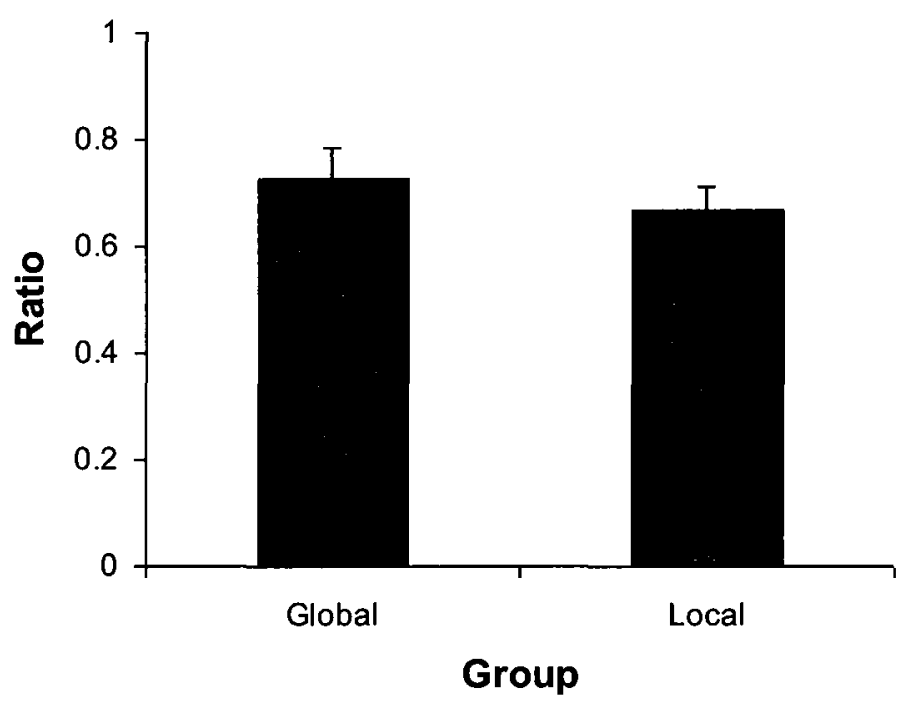

Figure 13. Ratio (+SE) of the number of landmarks included in map drawings for the Global and Local-only groups.

Landmark including by type and location. The impact of global versus local landmarks was investigated as a function of their location in the world, those located on the periphery versus internally. Therefore, the Both group was included in this separate analysis to investigate the difference between landmark inclusion based on both their type and location. If all landmarks are 'created equal' in terms of acquisition and recall, then no differences should be found between the types based on their locations. Planned paired sample t-test revealed that landmarks located in the periphery were recalled significantly more often than those located internally (see Figure 14) for the global, $t(19)=9.413, p$ $<.001$, and local landmarks $t(19)=2.235, p<.05$. Planned paired sample t-test revealed that global landmarks located in the periphery were recalled significantly more often than local ones, $t(19)=4.206, p<.001$ (see Figure 14). 


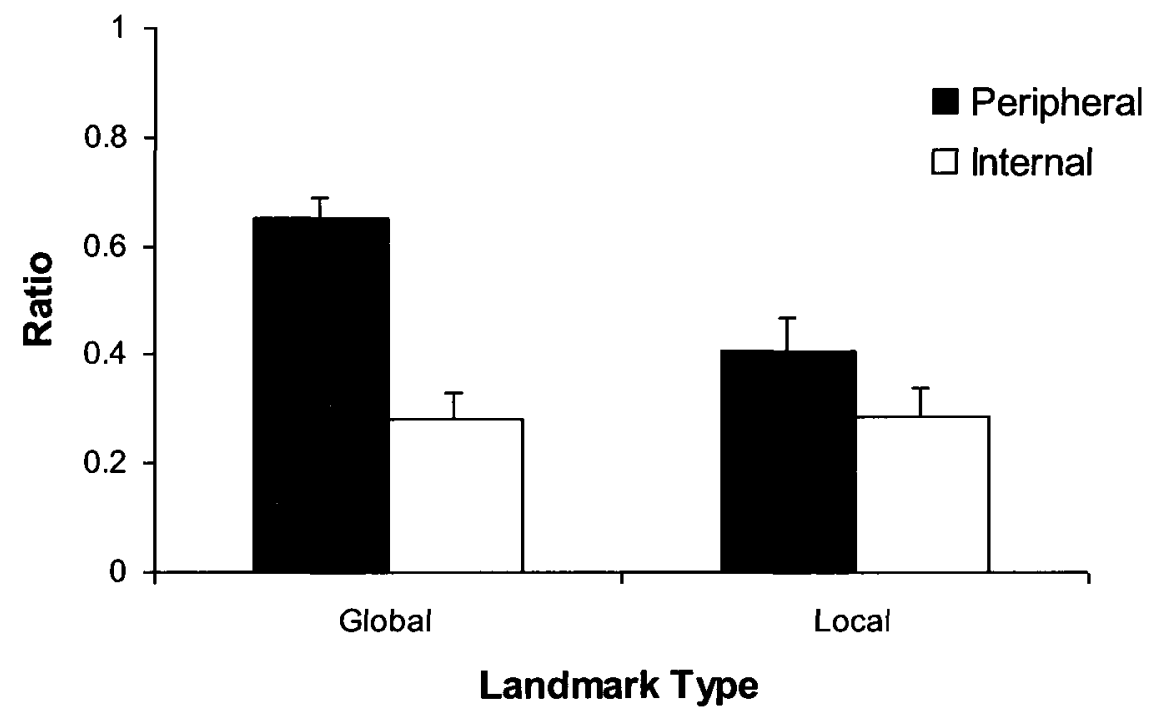

Figure 14. Ratio ( + SE) of the number of landmarks included in map as a function of landmark location for Global and Local-only groups.

Global landmark inclusion by location. Further analyses were performed to investigate the effect that the absence of local landmarks may have on sketch map landmark recall. To do this, sketch map performance was compared between the Both and Global-only groups. Differences found between these two groups may be attributed to the impact of the presence (Both group) or absence (Global-only group) of local landmarks. It was hypothesized that the presence of local landmarks would positively affect the Both groups' performance. Planned comparison independent t-tests revealed that the global group outperformed (i.e., a better ratio between number of landmarks available to number recalled) the Both group for the ratio of the number of peripheral 
$(t(38)=-3.765, p<.001)$, and internal $(t(31.057)=-3.261, p<.05)$ landmarks included ( see Figure 15).

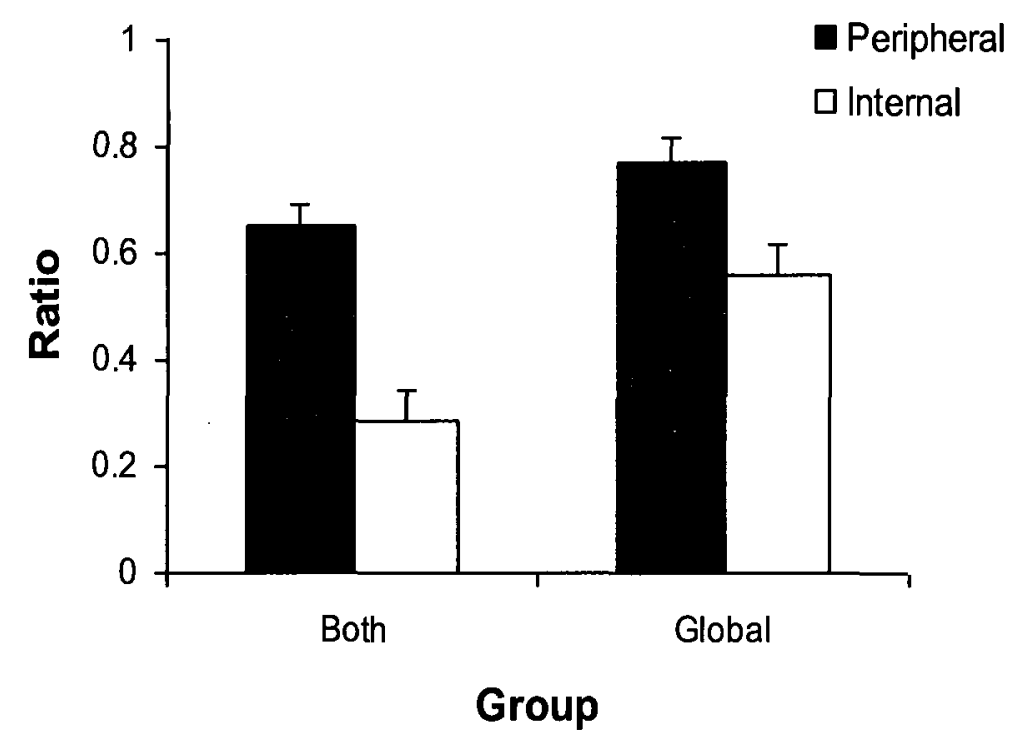

Figure 15. Ratio (+SE) of global landmarks by location included in map drawings for the Both and Global-only groups.

Local landmark inclusion by location. Further analyses were performed to investigate the effect that the absence of global landmarks may have on sketch map landmark recall. To do this, sketch map performance was compared between the Both and Local-only groups. Differences found between these two groups may be attributed to the impact the absence (Local-only group) or presence (Both group) that global landmarks may have on sketch map landmark recall. It was hypothesized that the absence of global landmarks would negatively affect the Local-only groups' performance when compared to the Both group. Planned comparison independent t-tests revealed that the 
local group outperformed the Both group for the number of peripheral $(t(38)=-3.251, p$ $<.05)$, and internal $(t(38)=-5.289, p<.001)$, landmarks (see Figure 16$)$.

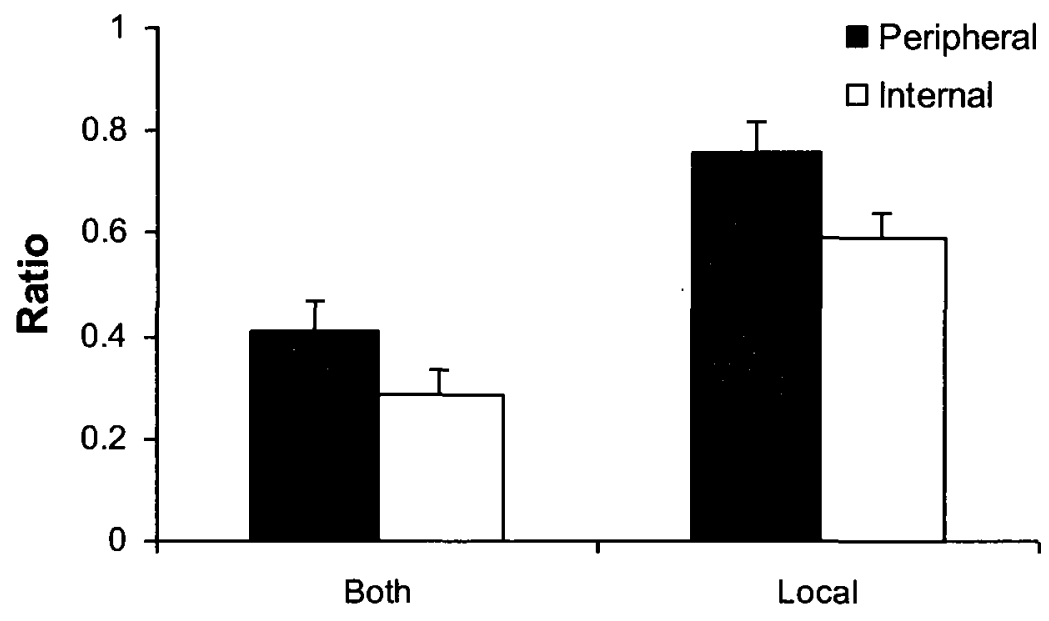

Landmark Type

Figure 16. Ratio (+SE) of local landmarks by location included in map drawings for the Both versus Local-only groups.

\section{Relative Landmark Placement}

A score of relative landmark placement in the sketch maps was used to provide a measure of differences in cognitive maps and landmark location knowledge. This differs from the previous measures of sketch map landmark inclusion as the latter entailed landmark recall only. Relative landmark placement incorporates whether participants placed landmarks accurately within the map relative to others. The relative landmark position scores have been used to compare across worlds (Billinghurst \&Weghorst, 1995) and argued to reflect survey knowledge. For each group, the five most frequently drawn 
landmarks were identified and relative positioning ratios were then calculated for each map (see Table 5). The ratio used was as follows:

Relative landmark placement ratio $=\#$ of correctly placed frequently drawn landmarks Total \# of frequently drawn landmarks (=5)

Table 5

The 5 Most Frequently Drawn Landmarks.

\begin{tabular}{ccc}
\hline Group & 5 Most Common Landmarks & Frequency \\
\hline Both & Ship & 20 \\
& Bridge & 19 \\
& Stadium & 19 \\
& Mountains & 16 \\
Global & Arch & 14 \\
& Bridge & 20 \\
& Stadium & 19 \\
& Ship & 19 \\
& Mountains & 18 \\
& Cathedral & 17 \\
& Heli-pad & 12 \\
& Traffic light & 12 \\
& Garbage can & 12 \\
& Vending machine & 10 \\
& Gas station or tennis court (tie) & 9 \\
& &
\end{tabular}


This list was used to calculate the relative landmark placement ratio which was in turn used to compare cognitive maps between groups. A one-way ANOVA was conducted to investigate differences across all groups. As global landmarks are hypothesized to help organize and structure the environment, it was hypothesized that the groups who received global landmarks would outperform the Local-only group. This analysis revealed a significant difference between groups, $F(2,56)=3.204, p<.05, \eta_{p}{ }^{2}=$ .110. Post hoc pair-wise comparisons revealed that the Both group performed significantly better at placing landmarks in the correct location when compared to the Local-only group, $p<.05$ (see Figure 17), but not significantly better than the Globalonly group. No difference was found between the Global and Local-only groups.

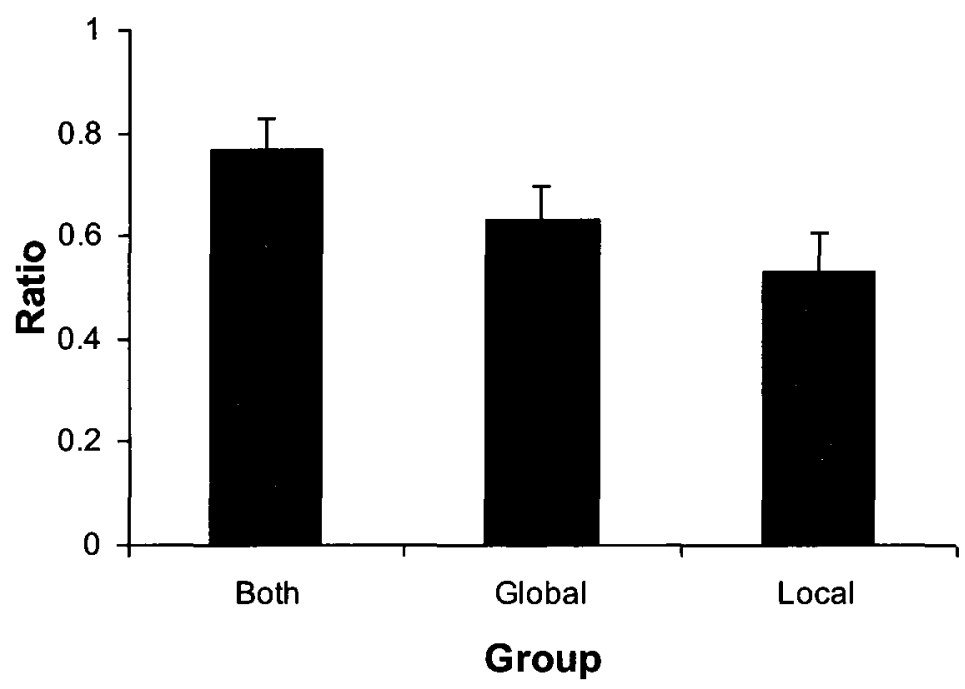

Figure 17. Relative landmark placement ratio (+SE) for all groups.

Of further interest to the analysis were the characteristics of the five most frequently drawn landmarks across groups. For future discussion two points should be 
noted. The first is that although all types of landmarks were available to the Both group, their five most recalled landmarks were all global peripheral landmarks with the majority (4 of the possible 5) being identical to those in the Global group. This is in line with the findings that global peripheral landmarks seem to be of utmost importance when recalling the world via sketch maps. The second point of interest is that out of the 15 most frequently recalled landmarks across all groups, $90 \%$ of these were landmarks located in the periphery, regardless of type. This finding will be investigated further in a later section.

\section{Map Goodness Ratings}

Maps were rated by three naïve raters to investigate qualitative differences of cognitive maps between groups via their sketch maps. A rating of 1 was labeled as 'useless' and 5 as 'extremely useful'. The three raters were found to have a significant intra-class correlation, indicating strong inter-rater reliability (.868). It was hypothesized that the maps of groups who had global landmarks available to them (Global-only and Both groups) would be rated significantly higher than the other groups. The reason for this is that global landmarks are hypothesized to provide structure and facilitate survey knowledge. A Kruskal-Wallis test revealed no significant differences between groups' mean map ratings.

\section{Way-finding Time as a Function of Location}

The map analyses conducted thus far have indicated that regardless of landmark type, peripheral landmarks are more quickly recalled. This finding sparked a question; could it simply be a result of participants spending more time at the periphery and thus 
encoding the peripheral landmarks more that the internal? To address this question, I investigated the amount of time participants spent on the peripheral roads versus those on the internal. The location participants were situated every 2 seconds while way-finding was written to a data file (as an $\mathrm{x}, \mathrm{z}$ coordinate pair). The data was split into two sets of $\mathrm{x}$ and $\mathrm{z}$ coordinates, one that included the coordinates of the peripheral roads and the second included $\mathrm{x}$ and $\mathrm{z}$ coordinates of the internal roads. Each participant's data file was analyzed for time spent by these two categorized locations. The dependent variable investigated was the natural logarithm of time spent in the periphery compared to time spent internally.

Given the previous sketch map findings, it was hypothesized that participants, regardless of group, were spending the majority of time in the periphery, explaining high rate of recall. A planned paired sample t-test found that regardless of group, participants were actually spending the majority of their time internally, $t(58)=-14.891, p<.001$ (see Figure 18). Descriptive statistics associated with this analysis can be found in Appendix L. 


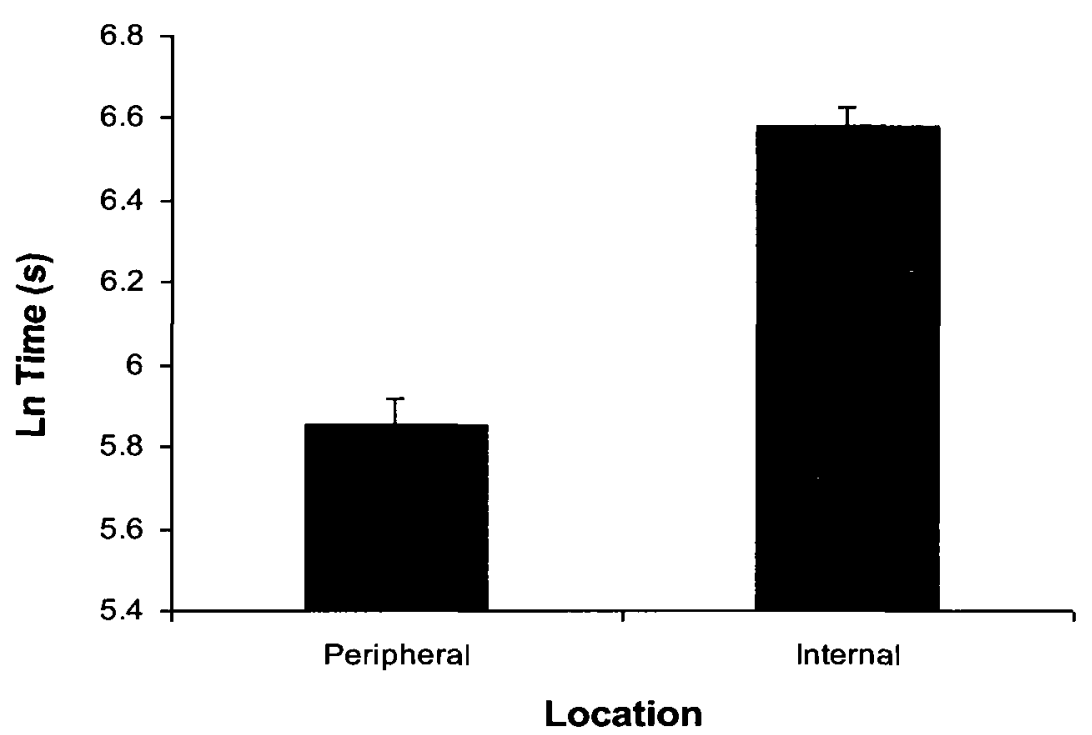

Figure 18. The natural log of time (s) (+SE) spent as a function of the location collapsed across all groups.

Differences between groups were also investigated by way of planned independent t-tests. It was hypothesized that the global-only group was spending a significantly longer time in the periphery, as this type of landmark was recalled most frequently. No differences were found between groups (see Figure 19). 


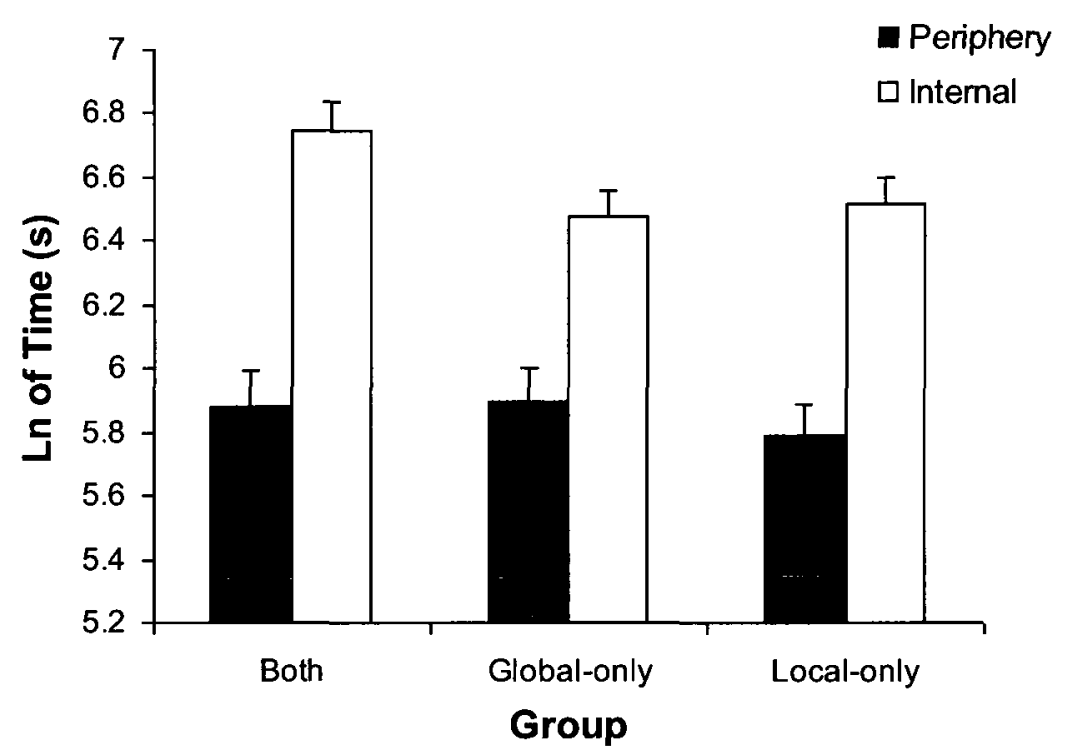

Figure 19. The amount of time spent in the world as a function of location (periphery vs internal) as measured by the Ln of time in seconds (+SE).

Four key findings were revealed by the sketch map analyses. First, the global and local only groups recalled an equal number of landmarks suggesting that the number of landmarks recalled is independent of the type that they are exposed to. Second, all three groups recalled more landmarks that were located in the periphery, which was not found to be based purely on the amount of exposure as the majority of their time was spent internally. Third, although both types of landmarks were available to the Both group, their five most recalled landmarks were all global landmarks located in the periphery, with the majority ( 4 of the 5 landmarks) being identical to those in the Global group. Finally, there were no group differences for the goodness of sketch maps. 


\section{Spatial Strategy Questionnaire}

Nine true and false questions were included in this questionnaire. The mean number of true and false answers to each question for each group was calculated. Chisquare tests were used to investigate if the groups' mean response to each question was significantly different than a true to false ratio of 50/50 (see Table 6).

Table 6

Spatial Strategy Questions: Proportion of True to False Questions for Groups

\begin{tabular}{|c|c|c|c|c|c|c|}
\hline \multirow[b]{2}{*}{ Question } & \multicolumn{3}{|c|}{$\frac{\text { Mean \# of True Answers }}{\text { Mean \# of False Answers }}$} & \multicolumn{3}{|c|}{$\begin{array}{c}\text { Chi-square test } \\
\text { statistic }\end{array}$} \\
\hline & Both & $\begin{array}{c}\text { Global- } \\
\text { only }\end{array}$ & $\begin{array}{l}\text { Local- } \\
\text { Only }\end{array}$ & Both & $\begin{array}{c}\text { Global- } \\
\text { only }\end{array}$ & $\begin{array}{l}\text { Local- } \\
\text { Only }\end{array}$ \\
\hline $\begin{array}{l}\text { I kept track of the direction in } \\
\text { which I was going. }\end{array}$ & $\frac{13}{7}$ & $\frac{14}{6}$ & $\frac{16}{4}$ & 1.80 & 3.20 & $7.20^{*}$ \\
\hline $\begin{array}{l}\text { I always kept in mind the point } \\
\text { from which I had entered the } \\
\text { world. }\end{array}$ & $\frac{7}{13}$ & $\frac{7}{13}$ & $\frac{7}{13}$ & 1.80 & 1.80 & 1.80 \\
\hline $\begin{array}{l}\text { I thought of my location in the } \\
\text { world in terms of North, South, } \\
\text { East and West. }\end{array}$ & $\frac{7}{13}$ & $\frac{5}{15}$ & $\frac{4}{16}$ & 1.80 & $5.00^{*}$ & $7.20^{\star}$ \\
\hline $\begin{array}{l}\text { Whenever I made a turn I knew } \\
\text { which direction I was facing. }\end{array}$ & $\frac{6}{14}$ & $\frac{9}{11}$ & $\frac{5}{15}$ & 3.20 & .20 & $5.00^{*}$ \\
\hline $\begin{array}{l}\text { I kept track of where I was in } \\
\text { relation to a reference point such } \\
\text { as the centre, water, mountain etc. }\end{array}$ & $\frac{18}{2}$ & $\frac{18}{2}$ & $\frac{17}{3}$ & $12.80^{\star \star}$ & $12.80^{\star \star}$ & $9.80^{*}$ \\
\hline As I went I made a mental note of & $\frac{3}{17}$ & $\frac{9}{11}$ & $\frac{3}{17}$ & $9.80^{*}$ & .20 & $9.80^{*}$ \\
\hline
\end{tabular}


the distance I traveled.

I visualized a map or layout of the

area in my mind as I went.

$\frac{17}{3} \quad \frac{14}{6}$

$\frac{13}{7}$

$9.80^{\star} \quad 3.20$

1.80

Objects were helpful to section the

world or find different areas

$\frac{20}{0} \quad \frac{19}{1} \quad \frac{20}{0} \quad n / a^{* *} \quad 16.20^{* *} n / a^{* *}$

within the world.

I counted the number of roads I

passed to help me find my way.

$\frac{10}{10}$

$\frac{9}{11}$

0.00

.20

3.20

$* \mathrm{p}<.05 ; * * \mathrm{p}<.001$

To analyze differences between groups, the mean proportion of "true" to "false" answers was calculated for each question. Chi-square tests revealed that regardless of group, landmarks were helpful to way-find and/or section the world and all groups kept track of where they were in relation to a reference point, such as the centre, water, mountain or other object. No significant group differences were found across the orientation and route related way-finding questions.

\section{Way-finding Decision Task (WDT) Performance}

Making correct way-finding decisions can reflect the level of acquired route knowledge. The WDT required participants to choose an action to reach a specific landmark along a route. The Both group received questions that referred to both global and local landmarks, Global-only group received questions that referred to only global landmarks and Local-only group received questions that referred to local landmarks. It was hypothesized that, since local landmarks are to have a positive impact on route 
knowledge acquisition, groups exposed to local landmarks would outperform those who received global landmarks only. The mean number of correct responses was used to test for differences in all WDT analyses. Descriptive statistics associated with WDT performance can be found in Appendix N.

A planned comparison independent t-test was used to test differences between the experimental groups. This test revealed no significant differences between the Globalonly and Local-only groups on WDT performance. Planned paired sample t-tests were used to test for Global-only and Local-only group differences within each group that may exist between landmark locations for WDT. No differences were hypothesized to exist. This test revealed that the Local-group made way-finding decisions more accurately with peripheral landmarks when compared to internal ones, $t(19)=3.217, p<.05$ (see Figure 20). No differences were found between landmark locations within the Global-only group. 


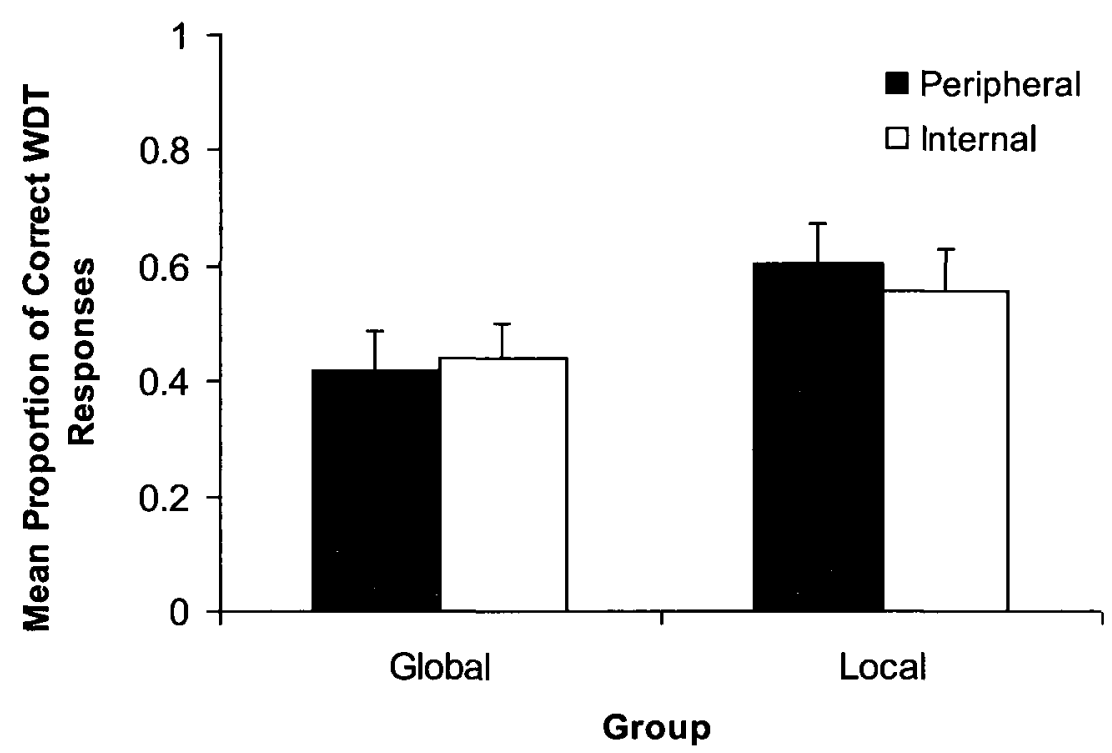

Figure 20. Way-finding Decision Task (+SE) performance for the Global and Local-only groups by landmark location.

WDT performance as a function of landmark type and location. The Both group was included in separate analyses to investigating the differences that may exist between WDT performance based on the combination of type and location of landmarks. It was hypothesized that performance in making way-finding decisions would be significantly better when referring to local landmarks, regardless of their location, as they are associated with way-finding and route knowledge. A planned comparison paired sample t-test was used to investigate differences. Performance for way-finding questions referring to local landmarks was significantly better than ones referring to global landmarks $t(20)=-3.122, \mathrm{p}<.05($ see Figure 21$)$ 


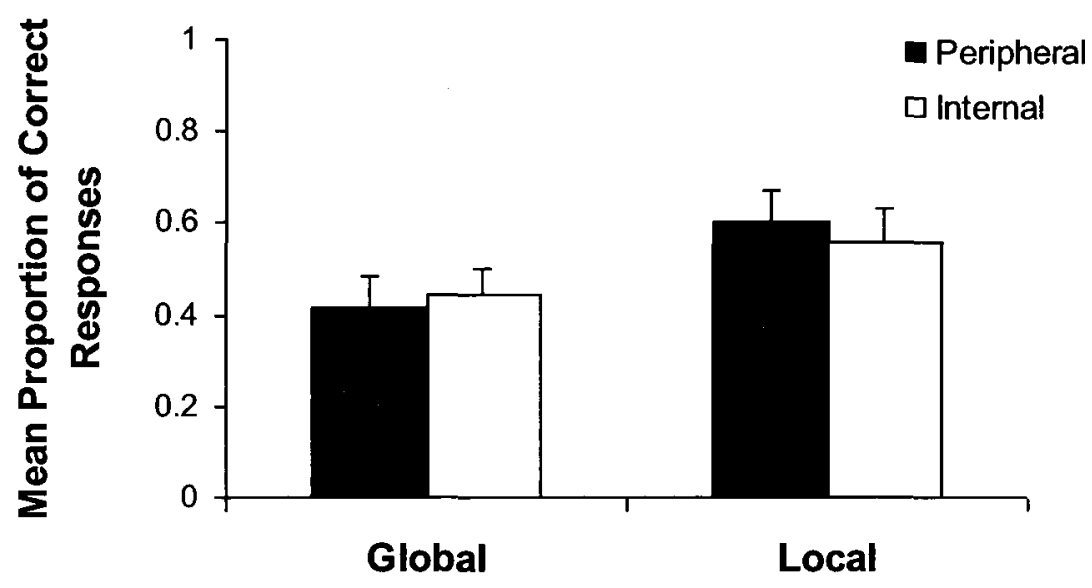

Landmark Type

Figure 21. Way-finding Decision Task $(+\mathrm{SE})$ performance for Both group by landmark type and location.

WDT performance as a function of global landmarks by location. The Both and Global-only groups were included in an analysis to investigate the impact of global landmarks and location on WDT performance. Differences found between these two groups can be attributed to the impact that the absence or presence that local landmarks has on WDT performance. Only those questions referring to global landmarks were included in this analysis, that is, all 12 WDT questions from the Global-only group and a subset of 6 of the 12 WDT questions from the Both group. The hypothesis that no group differences would be found was supported. No significant differences were found between the Both and Global-only groups on WDT questions referring to global landmarks overall or by its location. 
WDT performance as a function of local landmarks by location. The Both and Local-only groups were included in this analysis to investigate the impact of local landmarks overall as well as by location on WDT performance. Differences found between these two groups can be attributed to the impact that the absence or presence that global landmarks has on WDT performance. Only questions referring to local landmarks were included in this analysis, that is, all 12 WDT questions from the Local-only group and a subset of 6 of the 12 WDT questions from the Both group. The hypothesis groups would not differ was unsupported. Planned independent t-tests revealed that the local groups' performance for WDT questions referring to peripheral landmarks was significantly better than the Both groups', $t(39)=-2.577, p<.05$ (see Figure 22).

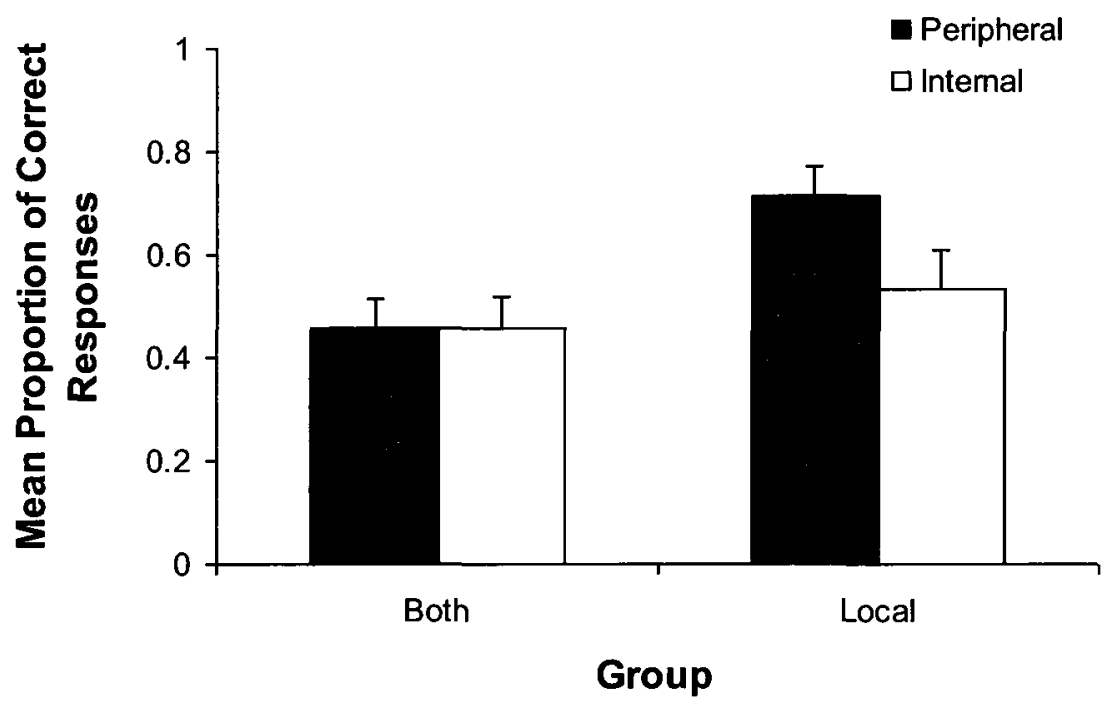

Figure 22. Mean proportion of correct WDT responses (+SE) for Both and Local-only groups for global landmarks by location. 
Overall, WDT performance was not dependent on whether global or local landmarks in isolation were provided. However, when both types are present way-finding questions in reference to local landmarks were answered more accurately when compared to global landmarks. Lastly, it was found that when provided with only local landmarks those in the periphery facilitate better WDT performance.

\section{Experiment 1: Discussion}

The aim of Experiment 1 was twofold: first, to investigate whether global and local landmarks have equivalent impact, independent of their location, on way-finding and spatial knowledge acquisition. The second aim was to investigate whether both types of landmarks together are essential for way-finding and spatial knowledge acquisition, or if spatial knowledge can be acquired in the presence of only one type. In general, the findings revealed that global and local landmarks do not have equivalent impact in both way-finding and spatial knowledge recall. Results suggest that precedence of landmark type is dependent on the amount of familiarity and spatial task. Findings also suggest that the presence of both types of landmarks is not essential for survey knowledge acquisition. Finally, the location of landmarks was found to play a central role in the recall of survey knowledge. The results from Experiment 1 are discussed in terms of the impact that landmark type (global and local) and location (peripheral and internal) has on spatial knowledge and way-finding.

The first key finding was that while way-finding, the impact of global and local landmarks in isolation was dependent upon experience or familiarity with the world. More specifically, global landmarks took precedence when the participant was naive to 
the environment; however with increased familiarity precedence for local landmarks was found. Additionally, when performance was investigated across familiarity, those exposed to local landmarks improved significantly, but those exposed to global only did not. These findings could be explained by the fact that the local-only group had very little exposure to their landmarks early in way-finding due to the nature of local landmarks' inferior visibility and thus required more time in the world to become exposed to the landmarks. Therefore, with increased exposure to local landmarks they become more familiar and thus more beneficial to complete the target search because of their impact on local way-finding decision making.

The opposite account can be made for the global landmark groups' superior performance early in the way-finding process. This could be the result of the shear visibility that global landmarks possess when compared to the local landmarks. The groups exposed to global landmarks had an advantage as they did not need to rely on amount of familiarity through the environment to way-find by way of global landmarks, as they were visible from most areas and could therefore be dependent on, and learned in a "passive", incidental way. However, if landmark visibility was the only explanation for differences found between the experimental groups, then no difference in performance between each group at the end of the way-finding task would be expected. This result was not the case and therefore leads to an alternate explanation.

If the impact of global and local landmarks is equivalent, significant differences in performance between the two experimental groups across time would not have been found. The fact that way-finding via global landmarks resulted in superior performance 
for naïve way-finders is important as it may provide insight into what type of information way-finders are trying to acquire in order to navigate more efficiently and reduce their chances of getting lost. In turn, this can provide insight into how spatial knowledge is processed, a stage-like process versus a more parallel processing strategy. As discussed in the introduction there is research that supports why global landmarks may be attended to early on in the spatial knowledge acquisition process as found in this experiment. Again, a defining characteristic of a global landmark is one that serves as a reference point, provides information that helps to structure the space, and provides distance or direction information.

The information associated with global landmarks and its importance to naïve way-finders can be explained by the Parallel Mapping Model (PMM). The PMM (Jacobs, 2003) argues that humans and animals have neural pathways to support two types of cognitive maps: one map is referred to as the bearing map, which is based on large scale directional information; the other, referred to as a local map, as one that is sensitive to fine grained local cues. Jacobs (Jacobs, 2003; Jacobs \& Schenk, 2003) suggested that each map plays a different role and may be sensitive to very different aspects of the environment, but with experience maps may become integrated with each other. This first experiment may have exposed two of the various features in the environment that these neural maps may be sensitive to. That is, the bearing map may be sensitive to landmarks based on their sensory cues, such as global landmarks, whereas the local map may be more sensitive to local landmarks. To complement Jacobs (2003) PMM, the current findings further suggest that how these two maps are acquired or built upon may be 
dependent on the type of landmark present to the way-finder. These findings indicate a significant importance of landmarks based on their sensory information, in this instance, their size.

Sketch maps provided insight into how landmark types might affect landmark and survey knowledge acquisition. The second key finding worthy of discussion is that survey knowledge acquisition can still occur when only one type of landmark is present, supported by the lack of group differences in overall sketch map goodness. The spatial strategy results also provide support that regardless of type of landmark available, landmarks, regardless of their type, were helpful to way-find or section the world. Further regardless of group, participants kept track of where they were going in relation to reference points such as the centre, water, mountain or other objects. This supports the argument that although the acquisition of specific types of spatial knowledge may be easier with either global or local landmarks, our spatial ability is adaptive enough that we can acquire the necessary knowledge with either type alone. Although the accuracy of survey knowledge as measured by sketch map accuracy did not seem to be dependent on landmark type the same cannot be said about landmarks by their locations.

Sketch map findings suggest that the spatial information, that is, the location associated with landmarks (as opposed to the sensory information only) plays a significant role when recalling elements and the layout of the space. Specifically, all groups recalled more landmarks that were located in the periphery when compared to those located internally, regardless of the type of landmark available. One possibility was that this finding may be related to the amount of time spent in the periphery and 
consequently amount of exposure to the landmarks located in the periphery. However, upon further investigation it was found that participants were spending a greater amount of time internally. It should also be noted that the group that had exposure to both types of landmarks recalled more global landmarks situated in the periphery. So, when given an option it could be argued that global landmarks located in the periphery are the easiest to remember or provide the most "useful" information when recalling an environment via sketch maps. In general, the results from the sketch maps indicate that both global and local landmarks situated in the periphery have a certain prominence within spatial memory during recall, with global landmarks playing a more central role when compared to local.

As a whole, the key findings from Experiment 1 support the claim that global landmarks may temporally precede local within way-finding, and play a central role within spatial knowledge recall when located in the periphery. The current findings, together with spatial cognition theories (Couclelis, et al., 1987; Jacobs \& Schenk, 2003; Lynch, 1960 ) lead towards the argument that global landmarks may provide "bearing" (as described by Jacobs, 2003) or "frame of reference" information (as described by Steck \& Mallot, 2002) that could potentially initiate the formation of a cognitive map through way-finding, as well as aid in recall of spatial knowledge. Results support a spatial knowledge acquisition process that originates with bearing or frame of reference information via global landmarks. As experience increases, local landmarks are integrated into the existing representation. 
In sum, the findings from Experiment 1 reveal that the impact global landmarks has is more substantial in naive way-finding, whereas the impact of local landmarks is more substantial with increased familiarity. While these findings can be explained by the PMM, I expand on this model by adding that the bearing and local maps are sensitive to global and local landmarks respectively. Additionally, positive impact for active wayfinding precedes with global followed by local, with local having a more positive impact with increasing familiarity. 


\section{Experiment 2: Isolated Impact of Landmark Location}

In Experiment 1 the isolated impact of landmark types in an attempt to reveal the roles that each plays in way-finding and spatial knowledge acquisition was investigated. The goal of experiment 2 was to isolate the impact of landmarks by their location. This goal was driven by the importance of landmark location found in the sketch map results in Experiment 1 and the need to isolate the impact addressed as a confound in previous research. Of particular interest is whether both landmark locations, peripheral and internal, have equivalent impact. In other words, does the absence of one location impact way-finding or spatial knowledge acquisition? One way to investigate the impact of landmark location would be to compare two groups, one with only peripheral and one with internally located landmarks. Although isolating the impact of landmark location is the primary focus, I felt it was important to also include the order in which global and local landmarks were available to the participants. This design was motivated by results from Experiment 1; the impact of landmark type was dependent on the amount of familiarity of the world during way-finding. Therefore, beyond investigating the isolated impact of landmark location I also investigated the impact of the temporal order in which landmark types were provided.

In the current study one group was exposed only to peripheral landmarks, while the other was exposed only to internal landmarks. Within each group, half of the participants were exposed to global landmarks only during the first half of their wayfinding trials followed by local landmarks for the latter half, and vice versa for the other half of each group. Again, the order in which participants were exposed to landmark type 
(global - local or local - global) was used to investigate the effect that the exposure to one type or the other during early and later spatial processing effects way-finding as suggested by findings in Experiment 1.

Based on the literature reviewed and discussed in the introduction, as well as support provided by the findings of the first experiment, It was hypothesized that peripheral landmarks, and more specifically global ones, would provide information that facilitates the acquisition of survey knowledge and mental structuring of the environment to aid in way-finding and spatial knowledge recall. If this hypothesis is supported further by Experiment 2, the group exposed to global peripheral landmarks should perform better in measures related to survey knowledge (e.g., map drawing), based on the rationale that global - peripheral landmarks may provide compass-like information, as suggested by (Steck \& Mallot, 1999). I further hypothesized that as local landmarks provide information that facilitates the acquisition of route knowledge, regardless of their location, groups (peripheral versus internal) performance should not differ.

\section{Method}

\section{Participants}

Eighty-seven undergraduate students (39 males, 48 females) were recruited via Carleton University's online psychology participant website and randomly assigned to one of two groups. Participants were given extra course credit or remuneration of $\$ 10 /$ hour for their participation, which took approximately 1.5 hours of their time. A short questionnaire regarding participants' health was completed and only those who 
were in good health and had normal or corrected-to-normal vision continued. The mean age of the population tested was 21 years $(\mathrm{SD}=4.50)$.

Apparatus and Materials

All desktop virtual worlds were built using the same tools as Experiment 1 . The four worlds used in Experiment 2 are described in Table 7. Please refer to Experiment 1, page 43 , for further information regarding the stimuli created for the spatial memory tests as well as the instructions presented as they were identical to those used in Experiment 1. Table 7

A Description of Each VE Used in Experiment 2.

\begin{tabular}{cc}
\hline \multicolumn{1}{c}{ World } & \multicolumn{1}{c}{ Description } \\
\hline Global-Peripheral & Contained 9 landmarks classified as global-peripheral. \\
Global-Internal & Contained 6 landmarks classified as global-internal. \\
Local-Peripheral & Contained 7 landmarks classified as local-peripheral. \\
Local-Internal & Contained 8 landmarks classified as local-internal. \\
\hline
\end{tabular}

\section{Study Design}

Participants were randomly assigned to one of two between-participant conditions, peripheral or internal. In each of these conditions, the order in which local and global landmarks were made available was also randomly assigned. Half of the participants in each group were first exposed to only local landmarks, at their respective location, followed by global, again at their respective location. The reverse order was presented to the other half of each group. The combination of landmark location and the order of exposure to landmark type resulted in four between participant groups: 
Group: Peripheral

Order 1: Global - Local: this group $(n=21)$ received 7 trials with the world that contained only global-peripheral landmarks, followed by the virtual environment that contained only local-peripheral landmarks.

Order 2: Local - Global: this group $(n=23)$ received 7 trials with the world that contained only local-peripheral landmarks, followed by the virtual environment that contained only global-peripheral landmarks.

Group: Internal

Order 1: Global - Local: this group $(n=23)$ received 7 trials with the world that contained only global-internal landmarks, followed by the virtual environment that contained only local-internal landmarks.

Order 2: Local - Global: this group $(n=20)$ received 7 trials with the world that contained only local-internal landmarks, followed by the virtual environment that contained only global-internal landmarks.

\section{Procedure}

As the majority of the procedure for the current experiment was identical to the procedure in Experiment 1, only items that differ are explained in this section. Upon arrival participants were brought into a quiet room and tested individually. Regardless of group (peripheral/internal) participants completed two conditions. Phase 1 consisted of 7 trials, followed by the primary spatial memory tasks (map drawing, WDT, and spatial strategy questionnaire) and secondary spatial memory tests (JRD, RDE). Once these tasks were completed the participants continued phase 2 way-finding, where they were to way- 
find in a second world to complete the remaining 8 trials, for a total of 15 trials. The second way-finding phase was followed by the primary and secondary spatial memory tests. Refer to Figure 23 for a schematic of the procedure used.

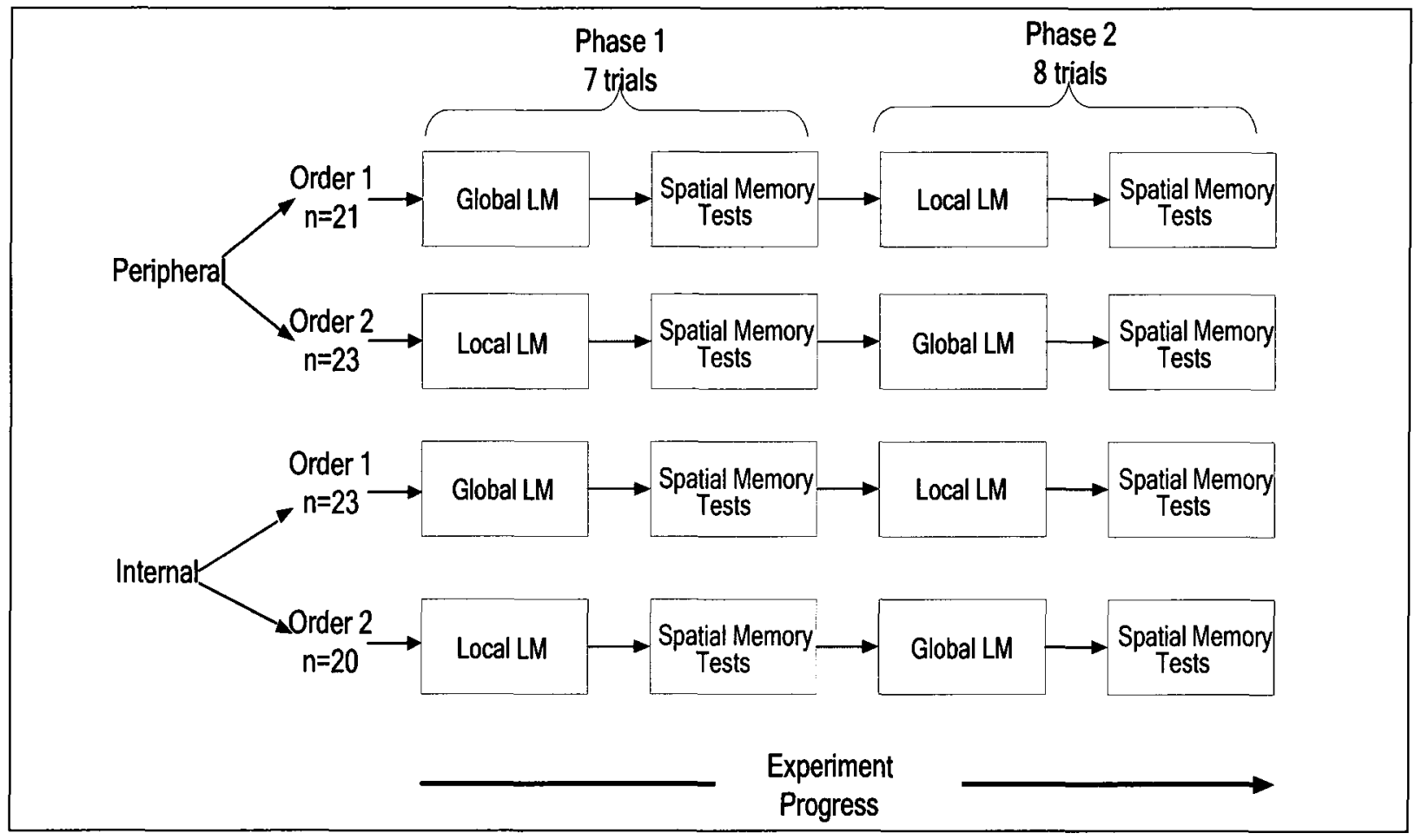

Figure 23. Overview of conditions experienced by the Peripheral and Internal Groups.

All spatial memory tests administered and measurements taken occurred twice in Experiment 2 (between the phases of each order and again after all 15 trials) and were similar to those administered in Experiment 1. The differences were only in the landmarks each question referred to, respective to each groups available landmark types and locations. Refer to Appendix $\mathbf{J}$ for a list of WDT questions. 


\section{Results}

The primary focus of this study was to investigate the impact of landmarks by their location on way-finding and spatial knowledge acquisition; with a secondary focus on the impact of the order of landmark type. Time to locate targets, WDT performance, and sketch maps were analyzed. All figures were presented with standard errors bars to aid in interpretation and descriptive statistics are provided in the associated appendices. Planned comparisons were administered to investigate comparisons between landmark locations and between the two orders (where appropriate).

\section{Way-finding and Target Search}

Participants were required to locate 15 targets in total; phase 1 contained 7 trials and phase 2 contained 8 trials. Phase 1 was divided into two blocks; with one block of 3 trials and one of 4 , the second phase was evenly divided into 2 blocks of 4 trials each. It was hypothesized that landmark location would not have an effect on way-finding. Further, given the results of Experiment 1, it was hypothesized that those in Order 1 (global landmarks followed by local) would perform significantly better than those in Order 2 for way-finding tasks. The natural log of time to locate targets was computed and used throughout all analyses. Descriptive statistics associated with way-finding performance for Experiment 2 can be found in Appendix $\mathrm{O}$.

Order 1 (global-local) by landmark location. It was hypothesized that groups based on landmark location at order 1 would not differ in their way-finding performance as there is no prior research to suggest so. Planned comparison independent t-tests were conducted. No differences were found to exist between the two groups at any block. 
Planned comparison paired sample t-tests for tests of performance within each group at each order. It was found that both the peripheral, $t(20)=3.887, p<.05$, and internal, $t(21)$ $=2.318, p<.05$, group's performance significantly improved between blocks 1 and 2 with no change after switching to local landmarks. A planned comparison independent ttest was used to investigate overall improvement as measured by the difference between the first and last blocks. It was found that the Internal group's overall performance approached significance, $t(22)=2.052, p=.052$ (see Figure 24 ). The break in the $x$-axis of Figure 23 represents the switch from global-only to local-only worlds.

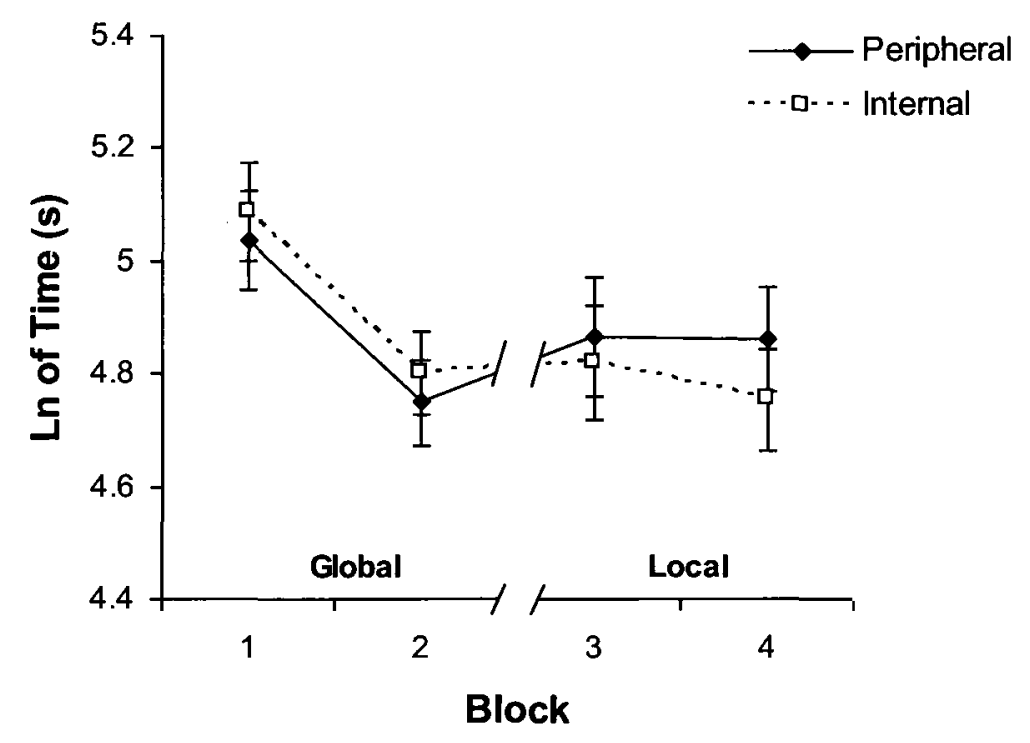

Figure 24. Way-finding performance as measured by the Ln of Time(s) (+SE) for Order 1.

The number of targets found by each group was compared to investigate whether there was a trade-off between the time to locate targets and the average ratio of the 
number of targets found per block. Ratios were used as there were only 3 targets to be found in the first block while all other blocks contained 4 targets. Based on results from Experiment 1, it was hypothesized that a trade-off would not be found. Differences between landmark locations when provided with global landmarks first followed by local landmarks were investigated. This test did not reveal any significant differences between groups at each block. Paired sample t-tests were used to investigate differences within each group which revealed that the Peripheral group's performance decreased significantly following the switch to local landmarks, $t(19)=-2.703, p<.05$ (see Figure 25). The break in the $x$-axis of Figure 24 represents the switch from global-only to localonly worlds.

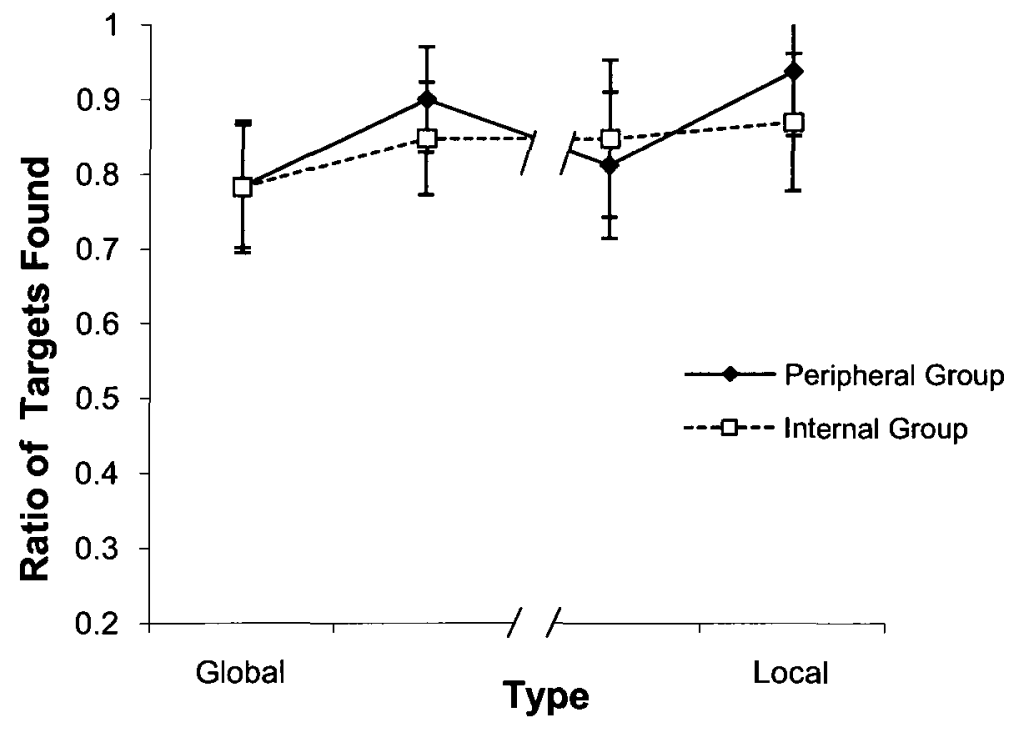

Figure 25. Way-finding performance as measured by the ratio of trials to targets found (+SE) by location for groups that received Order 1 . 
Order 2 (local-global) by landmark location. Again, there is no research or theoretical support to indicate that there should be any differences between the information provided by global or local landmarks that are located in the periphery or internally for way-finding. Therefore, it was hypothesized that groups based on landmark location at order 2 would not differ in their way-finding performance. Planned comparison independent t-tests were conducted. Differences between landmark locations when provided with local landmarks first followed by global ones at each of the four blocks were investigated by way of planned independent $t$-tests. This test did not reveal any significant difference between groups at individual blocks.

Order 1 (global-local) versus order 2 (local-global). Experiment 1 showed that performance was facilitated with exposure to global landmarks in early learning, and with exposure to local landmarks in later learning. Therefore, based on results from Experiment 1, it was hypothesized that order 1 would perform better than order 2 during the first phase and vice versa for phase 2 . This analysis is important as it will reveal if way-finding is also influenced by landmark location and not primarily by the type of landmark. If the results correspond to those found in Experiment 1, this could provide further support to the claim that global landmarks precede local in terms of attention or use in early way-finding regardless of its location. However, if the results do not correspond to Experiment 1, it can be argued that for global landmarks to precede local landmarks during early way-finding both locations and types are required.

Planned comparison independent t-tests were conducted to test for differences between groups at each block. At the second block it was found that the receiving global 
landmarks first resulted in significantly faster way-finding than those exposed to local landmarks first, albeit marginal, $t(85)=-1.963, p=.053$ (see Figure 26). Using planned paired sample t-tests to test performance within each group it was found that only order 1, global followed by local, showed significant improvement between the first and last block, $t(40)=2.925, p<.05$, regardless of the location of landmarks available. This same order, global followed by local, showed significant improvement between the first two blocks, $t(40)=3.293, p<.05$, that is, during their global landmark phase only, again regardless of the location. There was no significant improvement across time for the group that received local first followed by global. The break in the x-axis in Figure 26 represents the switch from global-only to local-only worlds.

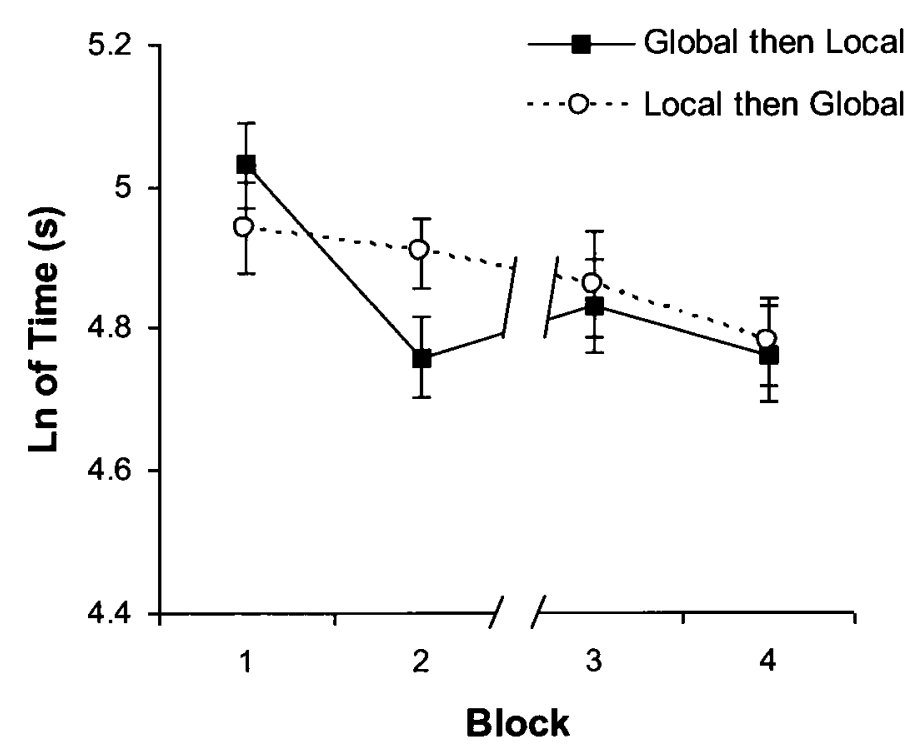

Figure 26. Way-finding performance as measured by the Ln of Time(s) $(+\mathrm{SE})$ for as a function of order. 
The possibility of a trade-off between the time spent locating targets and the number of targets found was investigated. Based on results from Experiment 1, I was hypothesized that a trade-off would not be found. Ratios were used as there were only 3 targets to be found in the first block while all other blocks contained 4 targets.

Differences between landmark orders collapsed across landmark locations were not found across any blocks, by way of planned independent $t$-tests. To investigate performance within each order planned comparison sample t-tests were used. When presented with local landmarks first performance did not significantly improve between blocks. However those that experienced global landmarks first and local second improved significantly across time, as measured by the difference between the first and last block, $t(43)=-2.725, p<.05$ as well as between the first two blocks (with global landmarks), $t(43)=-2.024, p<.05$ (see Figure 27). The break in the $\mathrm{X}$-axis of Figure 27 represents the switch from global-only to local-only worlds. 


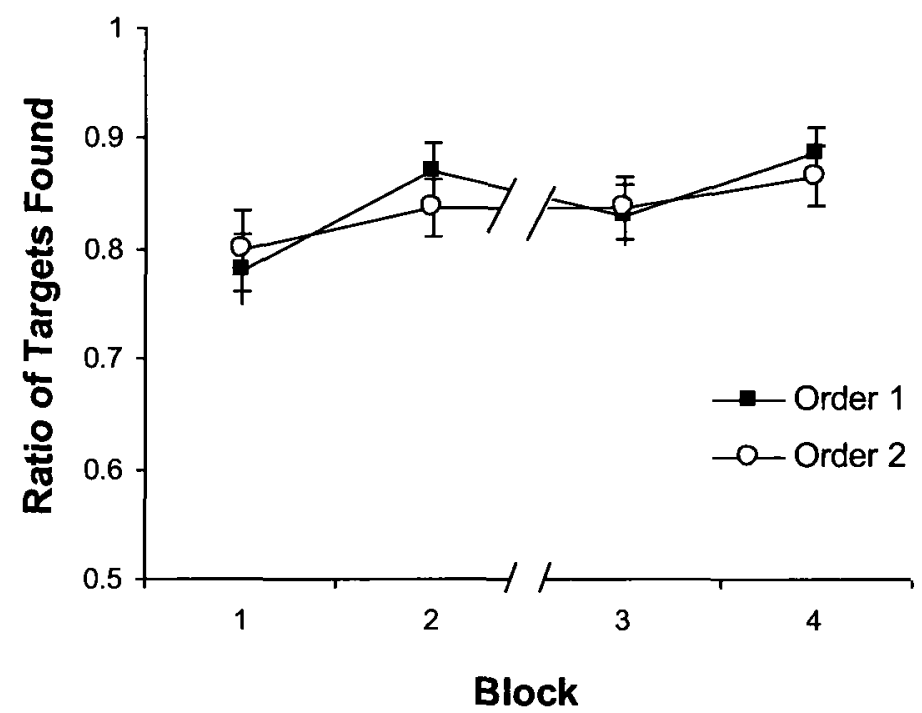

Figure 27. Way-finding performance as measured by the ratio of trials to targets found $(+\mathrm{SE})$ by Order.

Time spend by landmark location. Given Experiment 1's findings indicating that peripheral landmarks are recalled at a better rate, time participants spent on the peripheral roads versus the internal ones was investigated. The same method of analysis from Experiment 1 was used in Experiment 2. It was hypothesized that the location of landmarks provided would have an effect on the amount of time spent in each location. That is, when provided with peripheral landmarks, regardless of type, participants would spend more time in the periphery and vice versa. Planned comparison independent $t$-tests were used to investigate differences between groups and paired sample t-tests were used to investigate differences between conditions and time spent in each location overall 
regardless of group. These tests revealed that regardless of landmark location, a significantly longer time was spent internally, $t(56)=-22.776, \mathrm{p}<.001$ (see Figure 28).

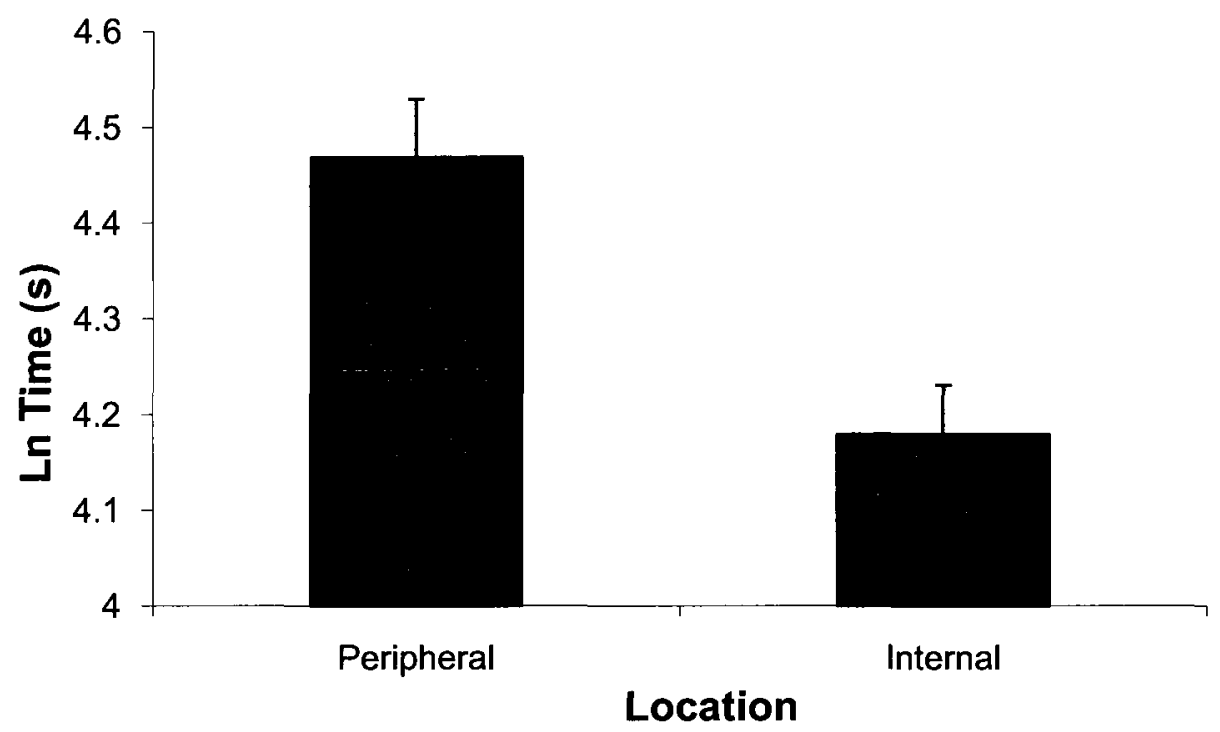

Figure 28. Amount of time spent in the world as a function of location (periphery versus internal) as measured by the Ln of time in seconds ( $+\mathrm{SE})$.

Three key findings resulted from the way-finding analyses. First, and consistent with Experiment 1, in the majority of way-finding results differences in performance were not found to be dependent on landmark locations. Second, those exposed to global landmarks first showed significant way-finding improvement, but no further improvement occurred once local landmarks were provided. Finally, groups exposed to local first showed no significant improvement across time even after global landmarks were provided. 
Spatial Memory Tests

Map analysis

The following sketch map analyses investigated group differences of survey knowledge including; the number of landmarks, whether they were placed correctly, and groups' survey knowledge as measured by 'map goodness' scores. To investigate differences in landmark recall at both locations, each map was analyzed in terms of the total number of landmarks present. The ratio of the total number of landmarks included in each sketch map over the total number of landmarks available in the virtual world was calculated for each map (i.e. two maps), one after each phase in the order, per participant. These ratios were used to control for the difference between the total numbers of landmarks available in each VE. Descriptive statistics associated with sketch map performance can be found in Appendix P.

Landmark recall at order 1 (global-local) by location. Differences between landmark recall for the Peripheral and Internal-only groups at Order 1 (presented with global followed by local landmarks) were investigated. It was hypothesized that the Peripheral group would include significantly more landmarks compared to the Internal group, regardless of type. A planned independent t-test revealed that the periphery group included significantly more landmarks overall, $t(41)=3.280, p<.001$. When investigated by landmark type, it was found that the Peripheral group included a significantly larger number of global landmarks when compared to the Internal group, $t(41)=5.122, p<.001$ (see Figure 29). 
Planned independent $t$-tests were used to investigate differences between landmark types that may exist within each group. It was hypothesized that regardless of landmark location participants would include more global landmarks. However, it was found that when presented with internal landmarks participants included a significantly larger number of local landmarks, $t(20)=-4.153, p=.001$ when compared to global (see Figure 29).

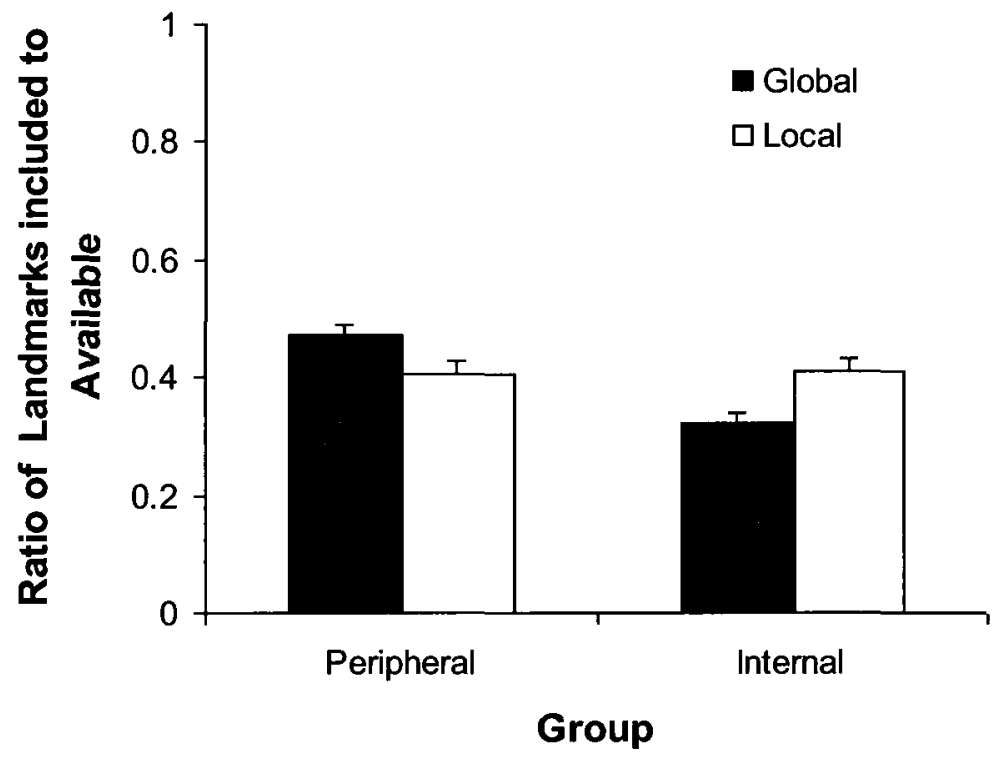

Figure 29. Ratio (+SE) of the number of landmarks included in map drawings to as a function of group at Order 1 .

Landmark recall at order 2 (local-global) by location. Differences were investigated between landmark recall for the Peripheral and Internal-only groups when presented with local followed by global landmarks. Given previous results, it was hypothesized that the Peripheral group would include significantly more landmarks 
compared to the Internal group, regardless of type. This test revealed that the Peripheral group did indeed include significantly more landmarks overall, $t(42)=3.415, p<.001$. When landmark type was investigated, it was found that the Peripheral group included a significantly larger number of global landmarks when compared to the Internal group, $t(35.496)=4.201, p<.001($ see Figure 30$)$.

Planned paired sample t-tests were used to investigate differences between landmark types that may exist within each group separately. It was hypothesized that participants in the Peripheral group would include more global landmarks compared to those in the Internal group given previous results. It was found that only the Peripheral group included a significantly larger number of global landmarks when compared to local, $\mathrm{t}(22)=3.172, \mathrm{p}<.05($ see Figure 30$)$. 


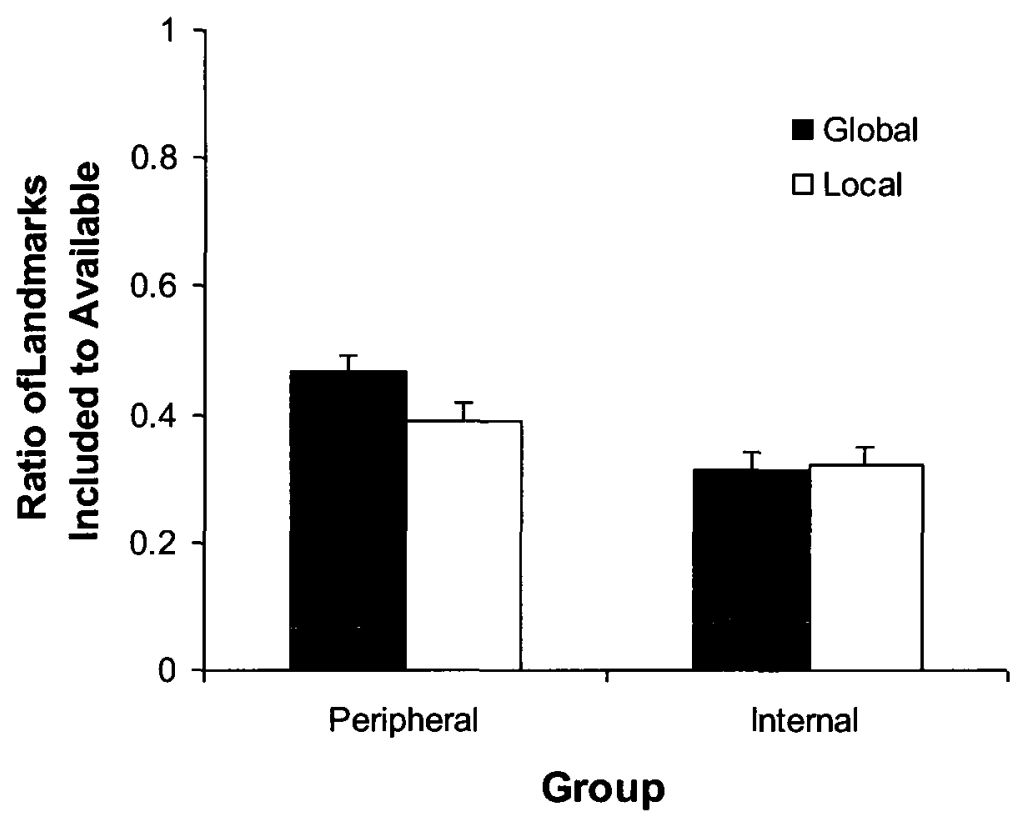

Figure 30. Ratio (+SE) of the number of landmarks included in map drawings as a function of groups as Order 2.

\section{Relative landmark placement}

A score of relative landmark placement for sketch maps was used to provide a measure of differences in cognitive maps and landmark location knowledge. This differs from the previous measure of landmark inclusion measuring only landmark recall, whereas relative landmark placement takes into account whether the participant included the landmark and placed its location accurately within the map relative to other landmarks. In previous research the relative landmark position scores have been used to compare across different worlds (Billinghurst \&Weghorst, 1995).

For each of the four worlds, the five most frequently drawn landmarks were obtained (see Table 8) and a relative positioning ratio was then calculated for each map. 
This list was used to calculate the relative landmark placement ratio which was in turn used to compare cognitive maps between groups. The ratio used was as follows:

Relative landmark placement ratio $=\#$ of correctly placed frequently drawn landmarks Total \# of frequently drawn landmarks $(=5)$

Table 8

The 5 most Frequently Drawn Landmarks by Group

\begin{tabular}{|c|c|c|c|c|}
\hline Group & $\begin{array}{l}5 \text { Most Common } \\
\text { Global Landmarks }\end{array}$ & Frequency & $\begin{array}{l}5 \text { Most Common } \\
\text { Local Landmarks }\end{array}$ & Frequency \\
\hline \multirow[t]{5}{*}{ Peripheral-Order 1} & Bridge & 21 & Vending Machine & 21 \\
\hline & Ship & 20 & Heli-pad & 21 \\
\hline & Stadium & 19 & Bus & 19 \\
\hline & Arch & 19 & Fountain & 18 \\
\hline & Cathedral & 17 & Car & 18 \\
\hline \multirow[t]{5}{*}{ Peripheral-Order 2} & Ship & 22 & Vending Machine & 20 \\
\hline & Bridge & 21 & Heli-pad & 20 \\
\hline & Stadium & 19 & Fountain & 20 \\
\hline & Mountain & 19 & Garbage Can & 19 \\
\hline & Arch & 17 & Car & 18 \\
\hline \multirow[t]{5}{*}{ Internal-Order 1} & Clock-tower & 19 & Pool & 21 \\
\hline & Roman Ruins & 19 & Construction Cones & 20 \\
\hline & Round Skyscraper & 17 & Traffic Light & 17 \\
\hline & Flags & 16 & Park/Gas (tie) & 16 \\
\hline & Crane & 16 & Station/Ladder & 16 \\
\hline
\end{tabular}




\begin{tabular}{lllll}
\hline Internal-Order 2 & Clock-tower & 16 & Construction Cones & 15 \\
& Tall Tower & 16 & Traffic Light & 14 \\
& Round Skyscraper & 16 & Pool & 14 \\
& Flags & 16 & Outhouse & 13 \\
& Crane & 16 & Gas Station/Ladder & 12 \\
\hline
\end{tabular}

Relative landmark placement by location at order 1 (global-local). It was hypothesized that the Peripheral group would correctly place a larger number of landmarks overall, and specifically a larger number of global landmarks correctly. Planned comparison independent t-tests were used to investigate differences between groups at order 1 and reveal the impact of landmark location on correctly placed landmarks by type. It was found that regardless of type, the Peripheral group placed a significantly larger number of landmarks correctly compared to the Internal group, $t(28.845)=3.039, p<.05$. When differences between types were investigated, it was found that the Peripheral group placed a significantly larger number of local landmarks correctly in their sketch maps, $t(22)=3.172, \mathrm{p}<.05$, when compared to the Internal group (see Figure 31). 


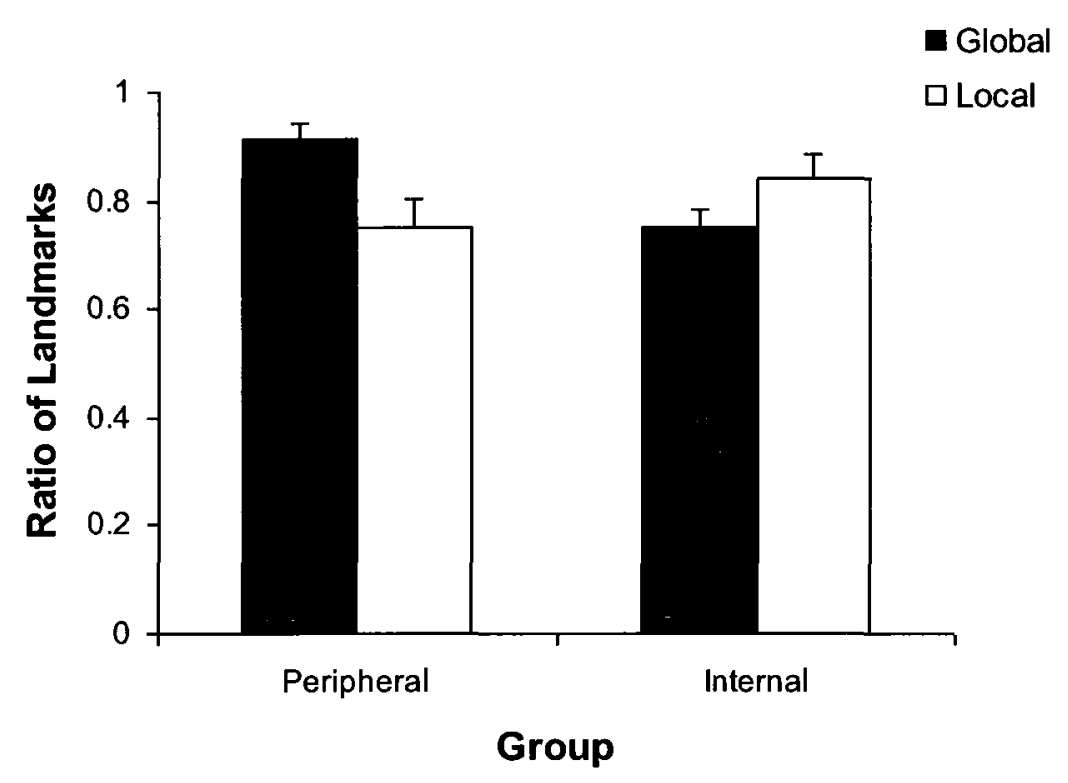

Figure 31. Relative landmark placement ratio $(+\mathrm{SE})$ for Peripheral and Internal groups at order 1 .

Relative landmark placement by location at order 2 (local-global). It was hypothesized that the Peripheral group would correctly place a larger number of landmarks overall, and regardless of location, participants would place a larger number of global landmarks correctly. Planned independent t-tests were used to investigate overall differences between the landmark location groups at order 2 (local followed by global) which would reveal the impact of landmark location on correctly placed landmarks by type. It was found that regardless of type, the Peripheral group correctly placed a significantly larger number of landmarks compared to the Internal group, $t(42)=3.110, p$ $<.05$. When differences between type were investigated, it was found that the Peripheral 
group placed a significantly larger number of local landmarks correctly in their sketch maps, $t(42)=3.384, p<.05$, when compared to the Internal group (see Figure 32).

Planned paired sample t-tests were used to investigate differences that may exist between landmark types within groups at condition 2. It was hypothesized that for both, the Peripheral and Internal groups, participants would place more global landmarks correctly within their sketch maps. It was found that the Internal group placed a significantly larger number of global landmarks correctly when compared to local, $t(19)$ $=-3.162, p<.05$ (see Figure 32).

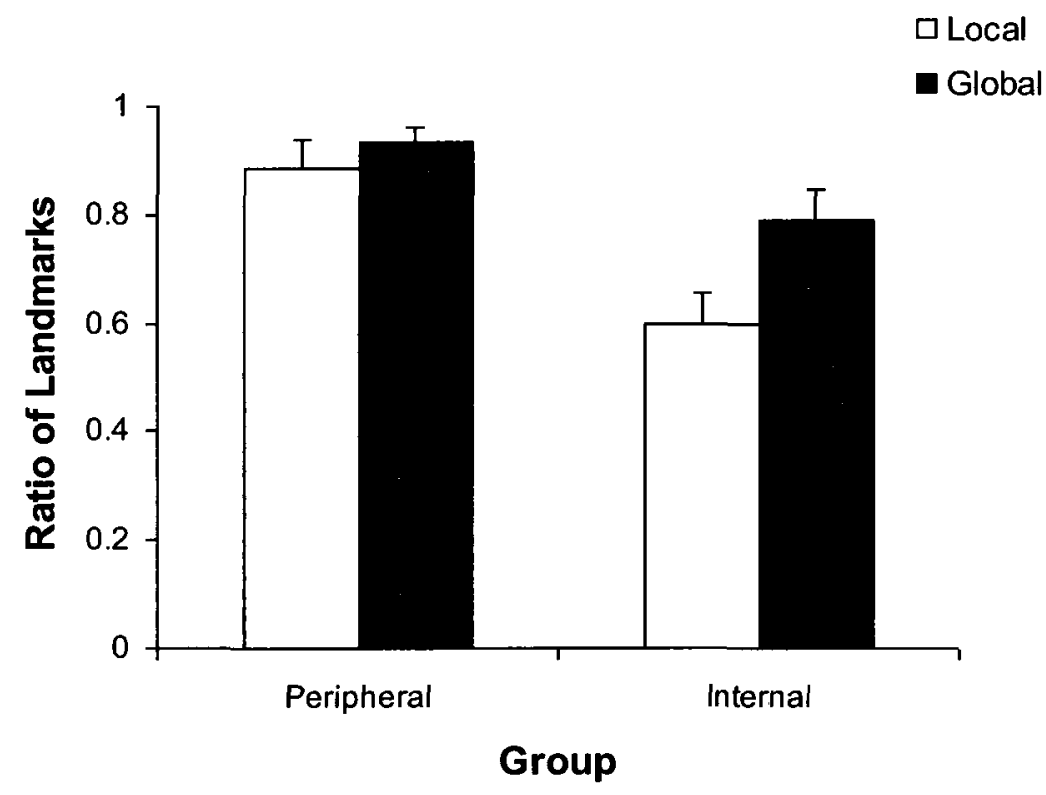

Figure 32. Relative landmark placement ratio (+SE) for Peripheral and Internal groups at order 2 . 


\section{Map Goodness}

Maps were rated by three naïve raters to investigate qualitative differences of cognitive maps between groups. Prior to investigating map "goodness" between groups the degree of inter-rater reliability was calculated. Even though the map ratings are highly subjective, the intra-class correlations coefficients (ICC) between the three raters was found to be significant for the global $(793, p<.001)$ and local $(.838, p<.001)$ maps within each group. An overall mean for the map ratings was calculated and used to find correlations between the map ratings, landmark recall and relative landmark placement scores. The Pearson Product Moment correlation were significant between map goodness and both landmark recall $(r=.477, p<.01)$ and relative landmark placement $(r=.524, p$ $<.01$ ), indicating a strong relation between the proportion of objects placed correctly in the drawn maps and landmark recall with mean map goodness ratings. Finally, a mean rating for type of map (global and local) for each group was calculated and a nonparametric Mann-Whitney $\mathrm{U}$ test was used to test for map goodness ratings between groups and within groups. The results are discussed next.

Map goodness ratings by location at order 1 (global-local). It was hypothesized that participants' maps in the Peripheral group would be rated better than the Internal group. Further, regardless of group, the maps for the virtual world with only global landmarks would be rated higher than the one with only local landmarks. At order 1 , when participants were exposed to global followed by local landmarks, the maps drawn by the group that was exposed to peripheral landmarks were rated as significantly better for their global maps, $(U=400.50, p<.001)$ when compared to the maps of the Internal 
group (see Figure 33). I also hypothesized that regardless of group, maps containing global landmarks would be rated better compared to maps containing local landmarks, however Wilcoxon rank sum test revealed no differences (see Figure 33).

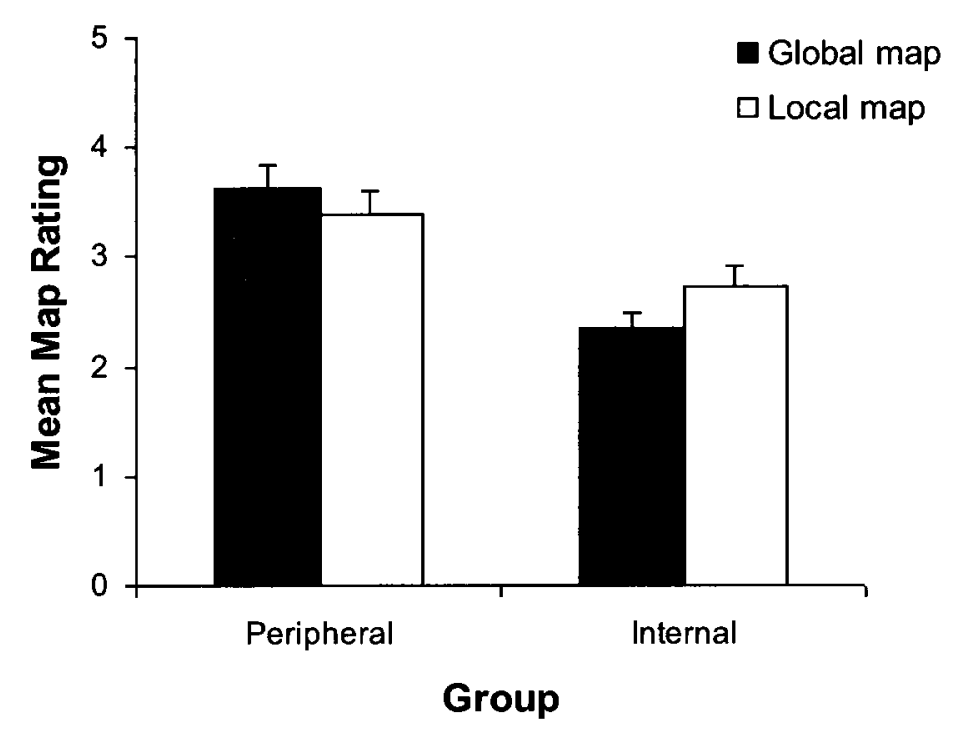

Figure 33. Map goodness rating ( $+\mathrm{SE})$ as a function of landmark type by Peripheral and Internal groups by order 1 .

Map goodness ratings by location at order 2 (local-global). It was hypothesized, based on findings from Experiment 1, that participants' maps in the Peripheral group would be rated better than the Internal group, and regardless of group the global landmark maps would be rated higher than the local. At order 2, when participants were exposed to local followed by global landmarks, the maps drawn by the group that was exposed to peripheral landmarks were rated as significantly better for their global maps, $(U=129.50, p<.05)$ when compared to the Internal group (see Figure 34). It was also 
hypothesized that regardless of group, participant's maps containing global landmarks would be rated better compared to maps containing local landmarks, however, only the Peripheral group had significantly higher map ratings for their global maps as measured by the Wilcoxon rank sum test $(p<.05)$ (see Figure 34 ).

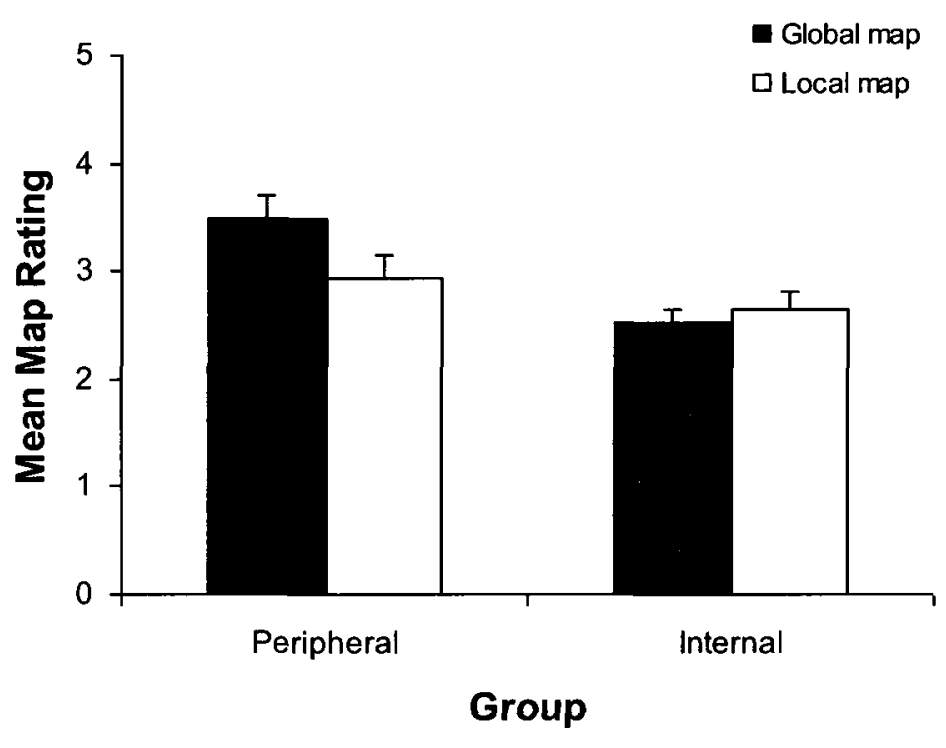

Figure 34. Map goodness rating (+SE) as a function of landmark type by Peripheral and Internal groups by condition 2 .

Two key findings from the sketch map analyses are noted but will be discussed in detail in the discussion section. First, the Peripheral group recalled and correctly placed more global landmarks, and their maps were given higher ratings when compared to the Internal group, regardless of order. Second, when provided only with internal landmarks participants remembered a significantly larger number of local landmarks compared to global. 


\section{Spatial Strategy Questionnaire}

The mean number of true and false answers to each question for each group was calculated. Chi-square tests were used to investigate the proportion of true to false responses for each question within each group (refer to Table 9, where "G" is used to denote global landmarks and "L" for local). Chi-square statistics associated with this questionnaire can be found in Appendix Q.

Table 9

Spatial Strategy Questions: Proportion of True to False Answers

\begin{tabular}{|c|c|c|c|c|c|c|c|c|}
\hline \multirow{4}{*}{ Question } & \multicolumn{8}{|c|}{$\begin{array}{l}\text { Frequency of True Answers } \\
\text { Frequency of False Answers }\end{array}$} \\
\hline & \multicolumn{4}{|c|}{ Order 1} & \multicolumn{4}{|c|}{ Order2 } \\
\hline & \multicolumn{2}{|c|}{ Peripheral } & \multicolumn{2}{|c|}{ Internal } & \multicolumn{2}{|c|}{ Peripheral } & \multicolumn{2}{|c|}{ Internal } \\
\hline & $\mathbf{G}$ & $\mathbf{L}$ & & $\mathbf{L}$ & & & & $\mathbf{G}$ \\
\hline \multicolumn{9}{|l|}{ I kept track of the direction in which I was } \\
\hline & $\underline{14}$ & $\underline{16^{*}}$ & $\underline{17}^{*}$ & $\underline{17}^{*}$ & $\underline{13}$ & $\underline{13}$ & $\underline{12}$ & $\underline{9}$ \\
\hline going. & 7 & 5 & 5 & 6 & 7 & 9 & 8 & 9 \\
\hline \multicolumn{9}{|l|}{ I always kept in mind the point from which } \\
\hline & $\frac{4^{*}}{17}$ & $\underline{7}$ & $\underline{8}$ & $\underline{5^{*}}$ & $5^{*}$ & $\underline{7}$ & $\underline{5}^{*}$ & $\underline{8}$ \\
\hline I had entered the world. & 17 & 14 & 15 & 18 & 17 & 15 & 15 & 10 \\
\hline \multicolumn{9}{|l|}{ I thought of my location in the world in } \\
\hline & $\underline{3 *}$ & $\underline{4^{*}}$ & $\underline{2 * *}$ & $\underline{4}^{*}$ & $\underline{2 * *}$ & $\underline{4 *}$ & $\underline{3 *}$ & $\underline{2 *}$ \\
\hline terms of North, South, East and West. & $\overline{18}$ & $\overline{17}$ & $\overline{21}$ & $\overline{19}$ & 20 & $\overline{18}$ & $\overline{17}$ & $\overline{16}$ \\
\hline \multicolumn{9}{|l|}{ Whenever I made a turn I knew which } \\
\hline & $\underline{14}$ & $\underline{8}$ & $\underline{10}$ & $\underline{7}$ & $\underline{7}$ & $\underline{12}$ & $\underline{6}$ & $\underline{6}$ \\
\hline direction I was facing. & 6 & 13 & 11 & 14 & $\overline{15}$ & $\overline{10}$ & $\overline{12}$ & $\overline{11}$ \\
\hline \multicolumn{9}{|l|}{ I kept track of where I was in relation to a } \\
\hline reference point such as the centre, water, & $\frac{14}{6}$ & $\frac{13}{8}$ & $\frac{17}{6}^{*}$ & $\frac{19}{4}$ & $\frac{16}{4}^{*}$ & $\frac{17}{4}^{*}$ & $\frac{12}{8}$ & $\frac{13}{5}$ \\
\hline \multicolumn{9}{|l|}{ mountain etc. } \\
\hline As I went I made a mental note of the & $\underline{14}$ & 1 & $\underline{8}$ & 7 & $\underline{10}$ & $\underline{9}$ & $\underline{6}$ & $\underline{10}$ \\
\hline & 6 & 14 & 14 & 16 & 12 & 13 & 12 & 7 \\
\hline
\end{tabular}


distance I traveled.

I visualized a map or layout of the area in

$\begin{array}{llllllll}\frac{19}{*}^{*} & \frac{13}{7} & \frac{20}{2}^{* *} & \frac{13}{9} & \frac{19}{3}^{* *} & \frac{22}{0 *} & \frac{13^{*}}{6} & \frac{16}{2}^{*}\end{array}$

my mind as I went.

Objects were helpful to section the world

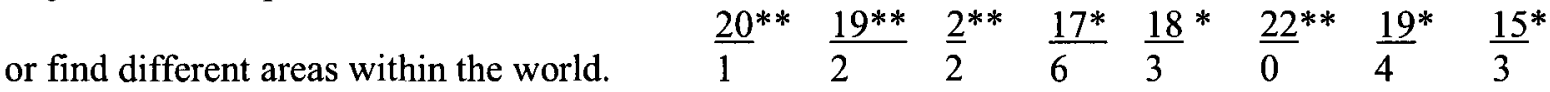

I counted the number of roads I passed to

help me find my way.

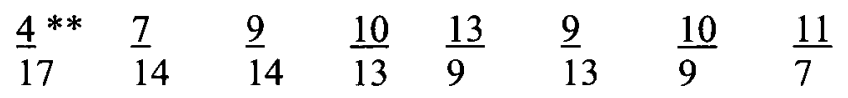

$* \mathrm{p}<.05$

$* * \mathrm{p}<.001$

To investigate differences between groups, the mean number of times each group responded "true" versus "false" was analyzed for each question separately. Chi-square tests revealed that regardless of group participants did not think of their location in the world in terms of North, South, East and West, however all groups indicated that landmarks were helpful to way-find and/or section the world.

\section{Way-finding Decision Task (WDT) Performance}

The WDT required participants to choose an action that would end in reaching a specific landmark along a route. It was hypothesized that when participants were exposed to local landmarks their performance would be significantly better compared to when they received global landmarks, regardless of landmark location (group). The mean proportion of correct responses was used to test the hypothesized differences in all WDT analyses. All descriptive statistics associated with WDT performance can be found in Appendix R. 
WDT performance by location at order 1 (global-local). Planned comparison independent $\mathrm{t}$-tests were used to test differences between performance when exposed to only peripheral and only internal landmarks at order 1. It was found that the Peripheral group's performance was significantly better than the Internal group when provided with local landmarks, $t(42)=2.337, p<.05$ (see Figure 35 ). No differences were found between landmark types within each of the groups.

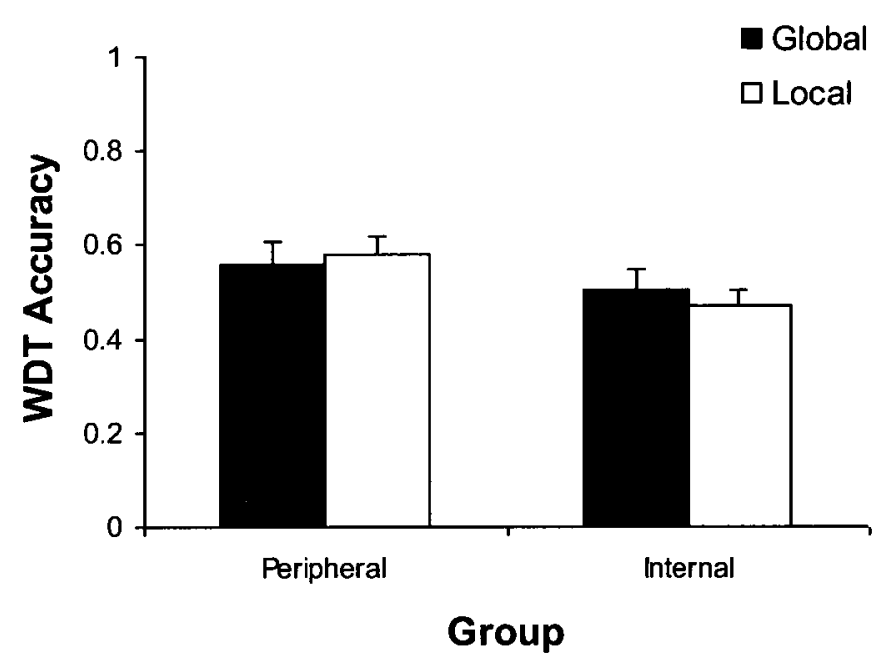

Figure 35. Overall WDT performance (+SE) for Peripheral and Internal groups when exposed to Global followed by Local landmarks.

WDT performance by location at order 2 (local-global). Planned comparison independent t-tests were used to test for differences in performance between those exposed to peripheral versus internal landmarks at order 2 . It was found that the Peripheral group's performance was significantly better than the Internal group when 
exposed to both local, $\mathrm{t}(41)=2.919, \mathrm{p}<.05$ and global, $\mathrm{t}(41)=2.353, \mathrm{p}<.05$ landmarks (see Figure 36). No differences were found within groups.

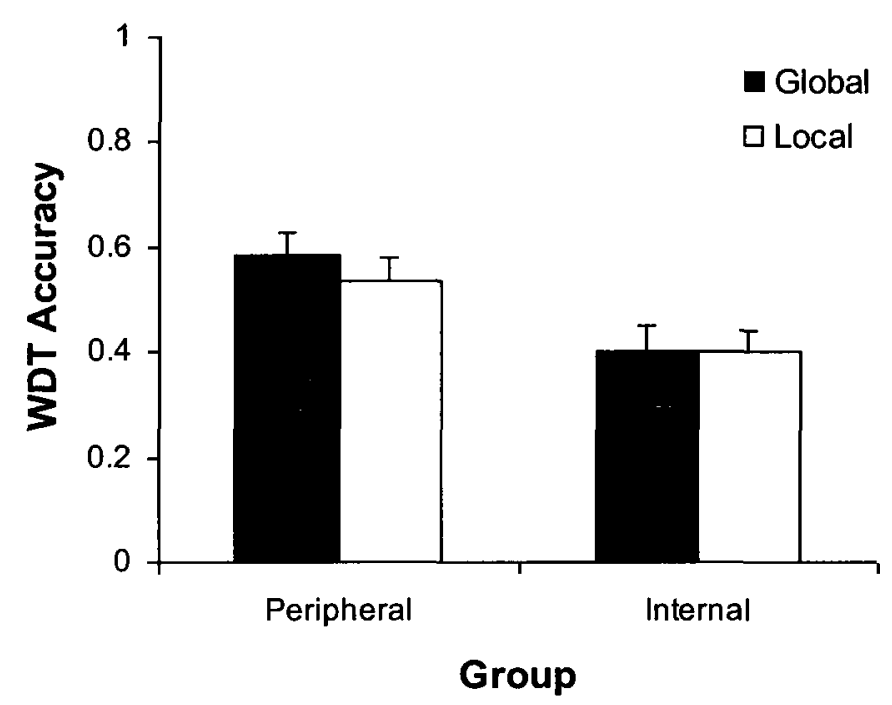

Figure 36. Overall WDT performance (+SE) for Peripheral and Internal groups when exposed to Local followed by Global landmarks.

WDT performance for order 1 versus order 2. Planned comparison independent ttests were used to test differences in performance between those exposed to landmark types at each order. It was hypothesized that those in order 2 would outperform those in order 1 as previous results have shown local landmarks may be most useful in wayfinding with increasing familiarity. No differences were found between or within orders for way-finding decisions referring to global or local landmarks. WDT analyses indicate that the Peripheral group's performance was significantly better at making way-finding decisions in reference to local landmarks at both orders and with global landmarks at order 2 when compared to the Internal groups' performance. 


\section{Experiment 2: Discussion}

The isolated impact of landmark location along with the temporal order of type on way-finding and spatial knowledge acquisition were investigated in this study. The results indicate that landmark type maintains a significant role when location is controlled for during way-finding. As in Experiment 1, this suggests that that the role of landmarks by their type is independent of its location for way-finding. Further support was found for a central role of landmarks by their location during survey knowledge recall, as in Experiment 1. Specifically, global landmarks located in the periphery and local landmarks located internally were found to have an impact on survey knowledge recall via sketch maps.

Consistent with results in Experiment 1, the presence of global landmarks resulted in a significant improvement in way-finding performance compared to local landmarks when unfamiliar with the environment. In Experiment 2, it was found that groups exposed to global landmarks first improved significantly, regardless of their location. Therefore, the additional contribution by Experiment 2 was that this finding was consistent regardless of where the global landmark was located, that is, internally or peripherally. Given this, I suggest that there is an existing precedence to attend to global landmarks when unfamiliar to an environment, as suggested by Lynch (1960), but additionally that this precedence is independent of location (Lynch, 1960). To generalize, this result provides further support, as stated in the discussion in Experiment 1, for the significant impact of landmarks' sensory information for way-finding. Furthermore, this impact is independent of their spatial information. 
Sketch maps results provide insight into the possible combined impact of landmark type and location in facilitating survey knowledge. Sketch map findings were consistent with those found in Experiment 1; that is, landmark location plays a central role when recalling survey knowledge. This argument is supported by the findings that those exposed to peripheral landmarks performed better than those exposed to landmarks located internally. Findings support the argument that exposure to global landmarks located in the periphery facilitates survey knowledge as measured by higher "map goodness" ratings, more landmarks recalled, and more correct relative landmark placement. In terms of the internal landmarks it was also found that when provided with global landmarks first, those exposed to internal landmarks recalled a significantly larger number of local landmarks. This may suggest an important role of local landmarks located internally when recalling an environment and its landmarks, but only once the global landmarks and their associated information have been acquired, as this finding was not persistent for the reverse order, local - global.

Taken together, the two key sketch map findings can be accounted for partially by the parallel mapping theory proposed by Jacobs (2003) discussed previously. The "parallel mapping model" suggests that the parallel acquisition of two dissociable maps may play different roles, and are each sensitive to different aspects of the environment. With experience, these two types of cognitive or neural maps can become integrated. Jacobs refers to one map as the 'bearing' map which is based on large scale directional information, and the other map as one that is sensitive to fine grained local cues. I suggest that landmark type and location play a combined role in facilitating the construction of 
bearing and local maps. Specifically, the superior recall of global landmarks in the periphery may reflect the construction and recall of the features in a 'bearing' map. Whereas the superior recall of local landmarks in the internal part of the world may reflect the construction of the local map. In terms of the internal landmarks, it was also found that when provided with global landmarks first, those exposed to internal landmarks recalled a significantly larger number of local landmarks. This suggests an important role of local landmarks located internally when recalling an environment. However, as this finding was only found in the global - local order, it is suggested that the "local" map can be built once global landmarks and their associated information has been acquired.

Experiment 1's findings suggest that the impact of global landmarks is more substantial when the environment is unfamiliar during way-finding, whereas the impact of local landmarks is more substantial with increased familiarity. The PMM was used to explain the results and was expanded upon by claiming that the bearing and local maps are sensitive to global and local landmarks respectively. Experiment 2 provides further evidence that the impact of global landmarks is more substantial when unfamiliar with the environment, whereas the impact of local landmarks is more substantial with increased familiarity. In addition, it is suggested that the impact of local landmarks on way-finding occurs only when following the acquisition of global landmark information.

When focusing on spatial knowledge, findings from Experiment 2 provide further elaboration on the PMM; affording the claim that the spatial information, that is the location associated with landmarks (as opposed to the visual information only), plays a 
significant role when recalling elements and the layout of the space via sketch maps. Therefore, if we focus on the two types of information, sensory and spatial that is associated with landmarks, we can acknowledge that there is some interdependency between the two types within spatial knowledge. Sketch map findings suggest that the spatial information, that is, the location associated with landmarks (as opposed to the sensory information only), plays a significant role when recalling elements and the layout of the space. Specifically, all groups recalled more landmarks that were located in the periphery when compared to those located internally, regardless of the type of landmark available. Therefore, we can acknowledge that way-finding is more dependent on the sensory information, regardless of its spatial information. 
Experiment 3: Landmark Type and Location as a Function of Familiarity In this final experiment I examine the roles of landmarks by their type and location in combination as a function of familiarity. Spatial familiarity can be described as how well a place is known (Gale, 1990). Therefore it can be assumed that the more familiar the VE is to the participant, the more accurately the cognitive map (Werner, Krieg-Brückner, Mallot, Schweizer, \& Freska, 1997). The aim of the final experiment was to gain insight into when landmarks are acquired and how they are integrated across levels of familiarity into our cognitive maps. This in turn can provide insight into the spatial knowledge acquisition process. Recall the stage-like LRS model and the more parallel school of thought as the two most commonly discussed theoretical approaches to account for the acquisition of spatial knowledge, which provides the foundation for the focal point of this final experiment.

To reiterate, the LRS model suggests that the landmarks (i.e. details) are initially learned followed by route and survey knowledge in a stage-like fashion (Siegel \& White, 1975; Yeap \& Jeffries, 2000). In contrast, others speculate that cognitive maps are formed in a more parallel fashion. When available, the global features are attended to first and then the details are "placed" within this representation (Aginsky et al, 1997; Lynch, 1960; MacEachren, 1992; Montello, 1998; Peponis, Zimring, \& Choi, 1990). Global landmarks are assumed to be associated with more survey related knowledge such as direction and distance estimation and aid in organizing and providing structure to the environment. Therefore it can be suggested that if global landmarks are learned prior to or in parallel with local, some level of survey knowledge may be acquired prior to the 
details. Conversely, if local landmarks are learned prior to global, along with their associated spatial knowledge, it would support the more traditional stage-like LRS theory of spatial knowledge acquisition.

Experiment 1 and 2 provided partial evidence that the roles of different types and locations of landmarks may change as a function of familiarity particularly within wayfinding. To date, the distinction between the different characteristics of landmarks and the roles they play within the process of acquiring spatial knowledge has not been the centre of any investigation, to my knowledge. Consequently, the objective of Experiment 3 was to study the impact of landmark type and location on spatial knowledge acquisition as a function of increasing levels of familiarity. Overall, if temporal precedence is found for either type or location of landmark based on familiarity this could provide novel insight into how landmarks based on their type and location are acquired and integrated into cognitive maps.

To investigate the development of spatial knowledge as a function of landmark type and location, participants completed spatial memory tasks at different levels of familiarity while way-finding. Levels of familiarity were investigated in a betweengroups design. A within-groups design was not chosen so as to control for any possible effects of repeated testing on performance. The difference between groups was based on the amount of familiarity with the VE, that is the number of trials was used as the between groups independent variable. The first group was tested after 5 trials (referred to as the 'novice' group), the second after 10 (referred to as the 'intermediate' group), and the third after all 15 trials had been completed (referred to as the 'advanced' group). In 
addition, a control group completed tests after all three levels of familiarity (after 5, 10 and 15 trials) to investigate the effect of repeated testing on spatial knowledge acquisition. It is hypothesized that precedence found for the acquisition of specific types and locations of landmarks will be reflected in performance between groups across familiarity on spatial memory tasks.

\section{Method}

\section{Participants}

Seventy-eight undergraduate students (39 males, 39 females) were recruited via Carleton University's online psychology participant website and randomly assigned to one of four groups. Participants were given extra course credit for their participation, which took approximately 2 hours. A short questionnaire regarding participants' health was completed and only those who were in good health and had normal or corrected-tonormal vision continued. The mean age of the population tested was 19.5 years $(\mathrm{SD}=$ 2.14).

Apparatus and Materials

The desktop virtual world used in this Experiment was identical to that used in Experiment 1 by the Both group which contained all 30 landmarks. All stimuli created for the experimental tasks as well as any instructions presented were also identical to those used Experiment 1 for the Both group.

Study Design

A between-participant design encompassed four levels of familiarity with the VE as the between-group independent variable. Participants were randomly assigned to one 
of four groups based on the amount of familiarity as measured by the number of trials completed. A summary of each group and when they were tested is provided below.

1. Novice: spatial memory tested following 5 trials, and again after the final $15^{\text {th }}$ trial,

2. Intermediate: spatial memory tested immediately following 10 trials, and again after the final $15^{\text {th }}$ trial,

3. Advanced: spatial memory tested immediately following the $15^{\text {th }}$ trial,

4. Control: spatial memory tested immediately following the $5^{\text {th }}, 10^{\text {th }}$, and $15^{\text {th }}$ trials.

\section{Procedure}

The procedure for the current experiment is identical to the procedure used in Experiment 1 and therefore only elements that differ are explained in this section.

Novice. Following 5 trials, the novice group completed the primary spatial memory tasks (map drawing, WDT, spatial strategy questionnaire) and secondary (JRD, RDE) spatial memory tests. Upon completion, participants were placed back in the world to finish the remaining 10 trials. Following a total of 15 trials the primary and secondary spatial memory tasks were repeated. Secondary spatial memory tasks are not included in the body of this dissertation. The questions, procedure and results associated with the secondary spatial memory tasks for Experiment 2 can be found in Appendix E.

Intermediate. Following 10 trials, the intermediate group completed the spatial memory tasks (map drawing, WDT, spatial strategy questionnaire). Once these tasks 
were completed the participants were placed back in the world to complete the remaining 5 trials. Following a total of 15 trials spatial memory tasks were repeated.

Advanced. Following 15 trials, this group completed the spatial memory tasks (map drawing, WDT, spatial strategy questionnaire).

Control. Following the 5th, 10th and 15 th trials, this group completed the spatial memory tasks (map drawing, WDT, strategy questionnaire) for a total of three times.

A summary of the groups and conditions is represented in Figure 37. Measurements obtained are identical to those obtained in previous experiments. Only results associated with the primary spatial memory tasks are presented in the results.

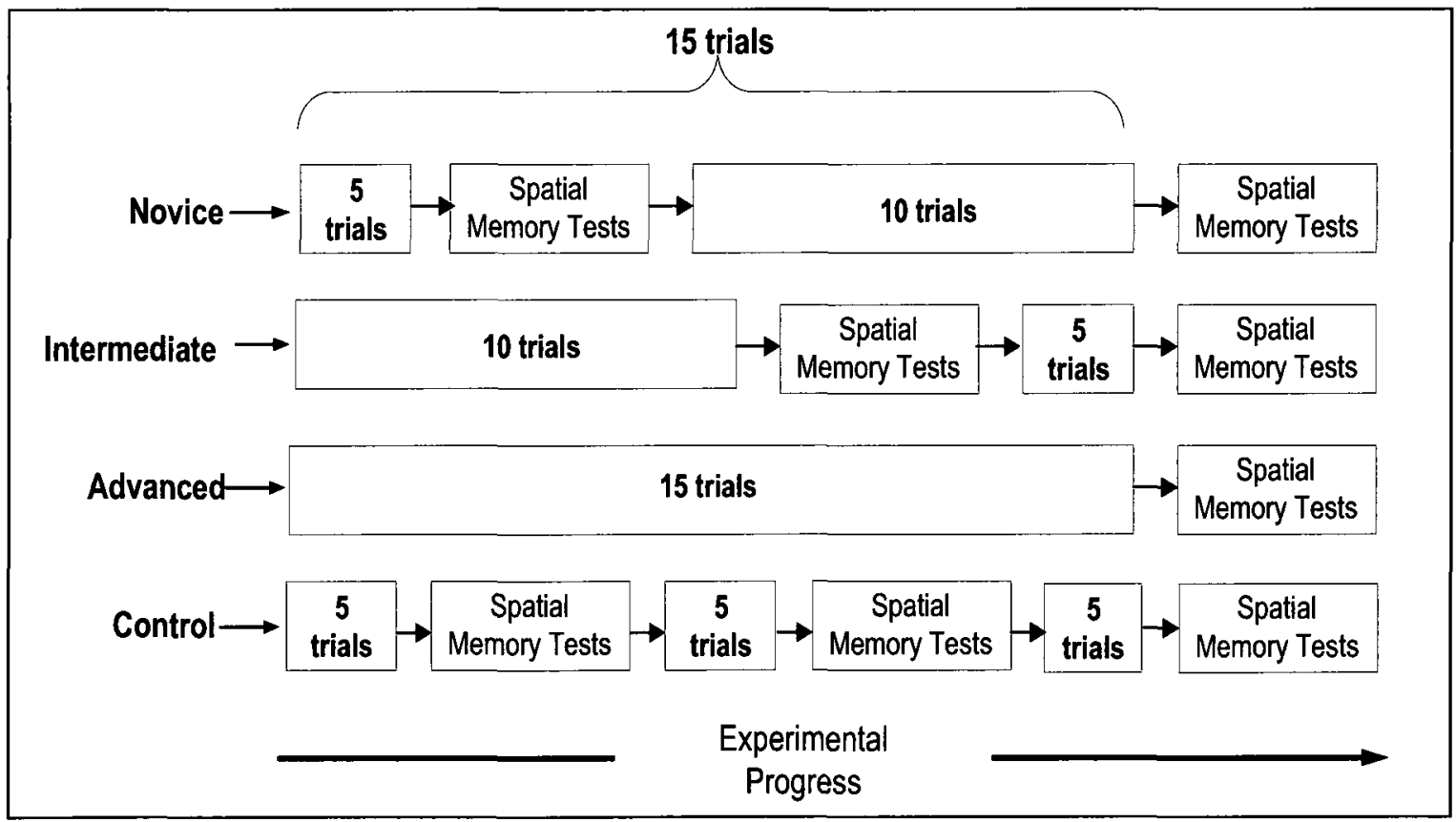

Figure 37. Schematic of Experiment 3's procedure. 


\section{Results}

Participants' time to locate targets, number of targets found, landmark and landmark location recall and sketch map goodness ratings, WDT performance, and spatial strategy questionnaire responses were analyzed. To examine and potentially control for confounding impact of spatial ability on performance, the MRT-A score was calculated. MRT-A was not used as a covariate as the scores did not differ significantly between groups and were not significantly correlated with any dependent variables.

\section{Learning Phase: Way-finding}

Participants were required to locate 15 targets over a series of 15 trials that were divided into three equal blocks. Time to find targets between blocks was analyzed to assess participants' performance across familiarity. I hypothesized that participants' time to find targets would improve across time for all groups. No group differences were hypothesized to exist at each block. This analysis does not provide any insight into how type and location of landmarks are integrated into our cognitive maps, but will afford the comparison of how the interrupted testing at various levels of familiarity may affect the trend displayed by groups. Descriptive statistics associated with the way-finding and target collection can be found in Appendix S.

To explore differences across familiarity and groups, a Repeated Measures ANOVA was performed on the transformed time to find targets. This test revealed a significant main effect of block, $F(2,140)=15.934, p<.001$, partial $\mu^{2}=.185$ (see Figure 38). No difference between groups was found. The post hoc paired sample t-tests indicated that, regardless of group, performance significantly improved between the first 
and second block, $t(74)=3.249, p<.017$, the first and third block, $t(73)=6.099, p<.001$, and marginally between the second and third block, $t(73)=2.381, p<.020$. Overall, results indicate that, regardless of group, improvement to locate targets as a function of familiarity in the environment was exhibited. This indicates that testing at the various levels of familiarity does not affect the trend displayed by all groups.

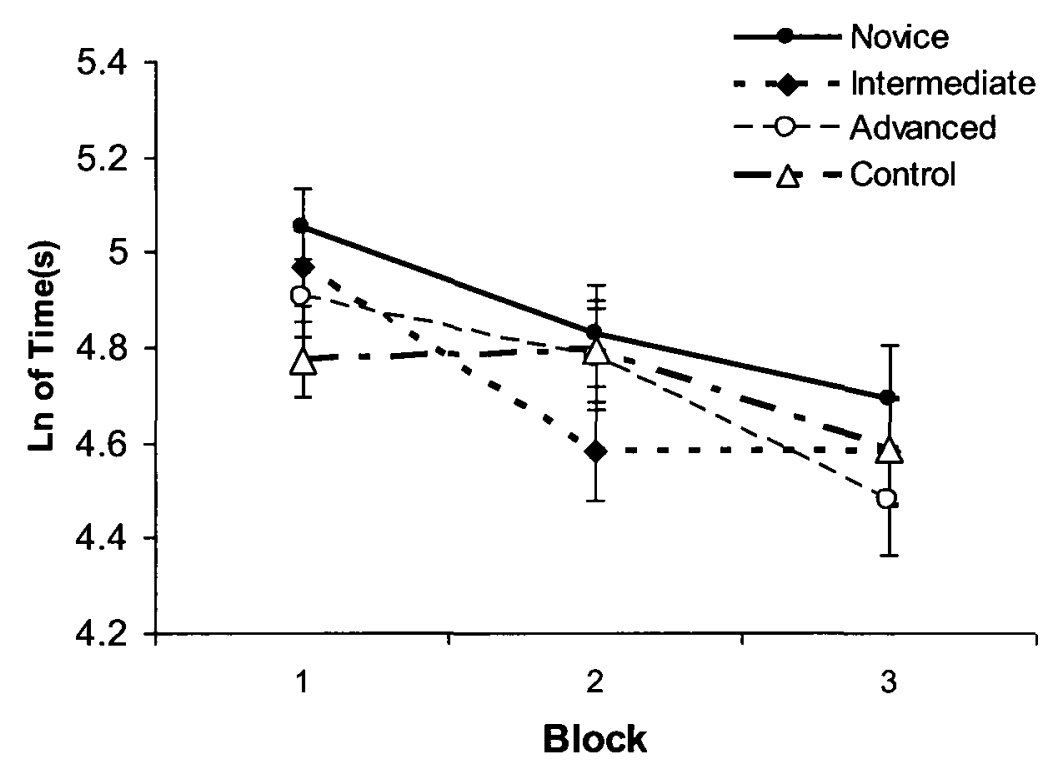

Figure 38. Mean time to locate targets (+SE) as a function of group across blocks.

\section{Time Spent by Location}

Way-finding analyses conducted in both Experiments 1 and 2 indicated that more peripheral compared to internal landmarks are recalled and placed correctly within sketch maps. Therefore, the amount of time participants were spending way-finding on the peripheral roads versus those on the internal was investigated. The location participants were situated every 2 seconds while way-finding was written to a data file (as an $\mathrm{x}, \mathrm{z}$ 
coordinate pair). The data was split into $\mathrm{x}$ and $\mathrm{z}$ coordinates that were located on the peripheral and $\mathrm{x}$ and $\mathrm{z}$ coordinates on the internal roads, and each participant's data file was analyzed for time spent by location. Given the previous sketch map findings it was hypothesized that the majority of participants' time was spent internally. The dependent variable investigated was the natural logarithm of time spent in the periphery and internally. A planned paired sample t-test found that regardless of group participants were spending the majority of their time internally, $t(58)=-24.448, p<.001$ (see Figure 39).

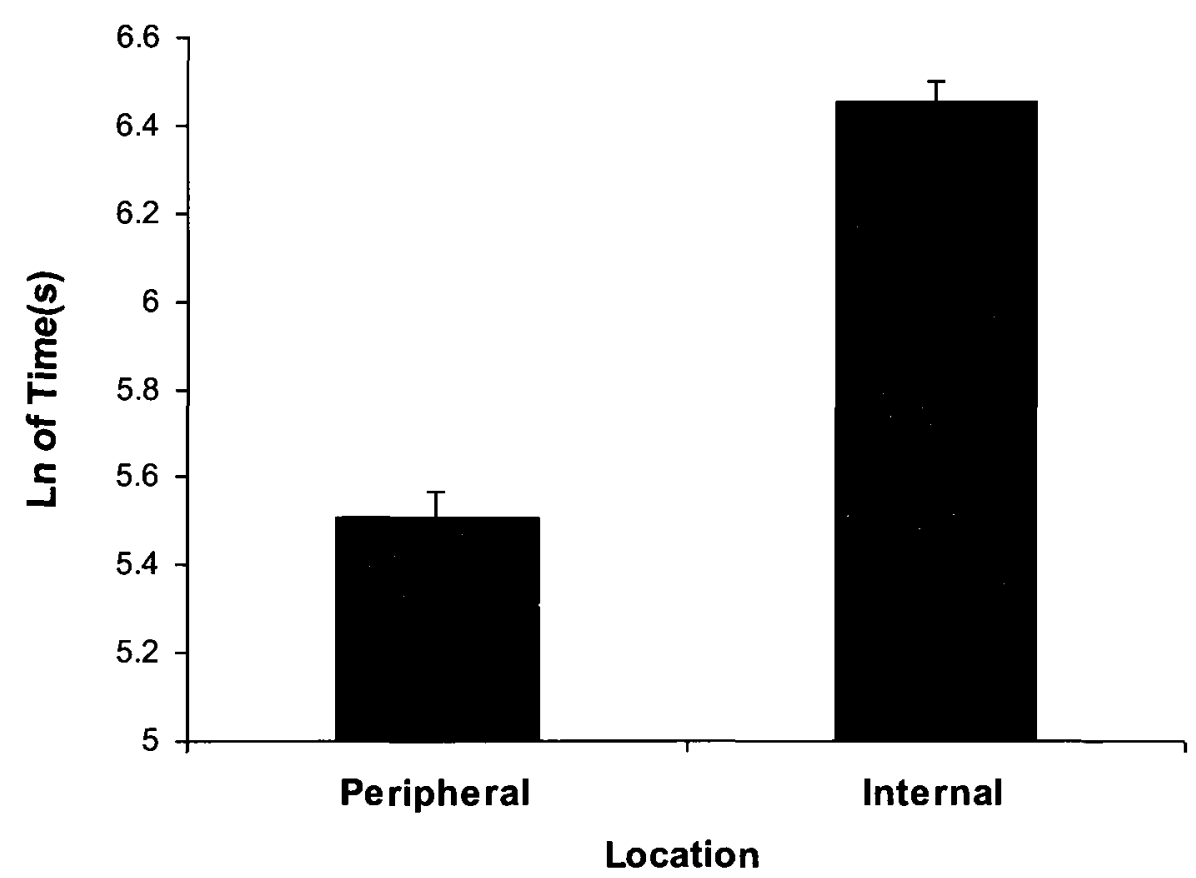

Figure 39: The amount of time spent in the world as a function of location (periphery versus internal) as measured by the Ln of time in seconds (+SE) by group. 
Spatial Memory Tests

Sketch Map Analysis

The following analyses investigating group differences of the number of landmarks recalled, the correct placement of the landmarks, and overall map 'goodness' to assess groups' survey knowledge. Descriptive statistics associated with the sketch map analyses can be found in Appendix T.

Each map was analyzed in terms of the total number of landmarks to investigate differences between the type and location of landmarks as a function of familiarity. Included in this analysis is the total number of landmarks regardless of type or location, total number of landmarks by each type, and total number of landmarks by each location. The final analysis included in this section was an analysis comparing type and location in combination. Descriptive statistics associated with the sketch map analyses can be found in Appendix T.

Total landmarks included by familiarity. It was hypothesized that the as the level of familiarity increases so to would the number of landmarks recalled. The group means were calculated and compared to investigate differences at the three levels of familiarity using independent $\mathrm{t}$-tests, represented by the dotted line in Figure 40. This analysis did not reveal any significant group differences for the total number of landmarks recalled (see Figure 40). However, when the control group was investigated, using planned paired sample t-tests, a significant improvement between block 1 and $2, t(18)=-5.441, p<.000$, as well as block 2 and $3, t(18)=-5.745, p<.000$, was found. Planned comparison t-tests revealed a significant difference between the Advanced group (tested once after all 15 
trials) and the Control group at the last block (see Figure 40), $t(37)=2.520, p<.05$. Two explanations for the different results found between the Advanced and Control group at block 3 are provided. The control group may have experienced a learning effect as a result of multiple testing, resulting in significantly more landmarks recalled following each testing phase. If this is the case it justifies the use of the chosen experimental design as it was the intent to test the impact of familiarity but without the confounding effect of repeated testing. A second explanation could have been that spatial ability, gaming experience, or age for the Expert group was significantly different from the other Experimental groups however this was not the case (see Figure 40).

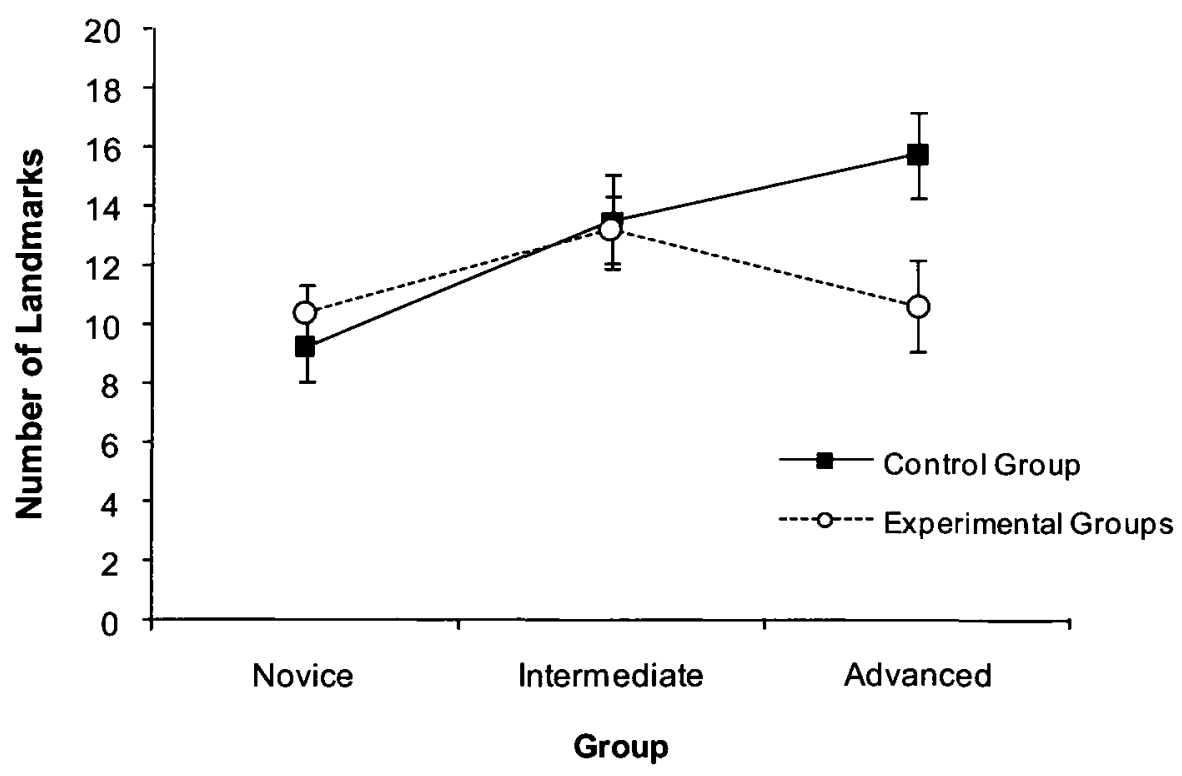

Figure 40. Total number of landmarks recalled (+SE) (i.e. included in sketch maps) across familiarity as a function of Experimental and Control groups. 
Landmarks included by familiarity as a function of type. Given the way-finding results in the first two experiments and related literature, I hypothesized that a larger number of global landmarks would be included earlier in learning compared to local.. Planned paired sample t-tests revealed that those with only novice $(t(19)=-3.231, p<$ $.05)$ and intermediate familiarity $(t(18)=-3.835, p<.05)$ recalled a significantly larger number of global landmarks compared to local (see Figure 41). However, differences between global and local landmark recall were not found in those with more advanced familiarity. No significant increase in the number of global or local landmarks across familiarity was found.

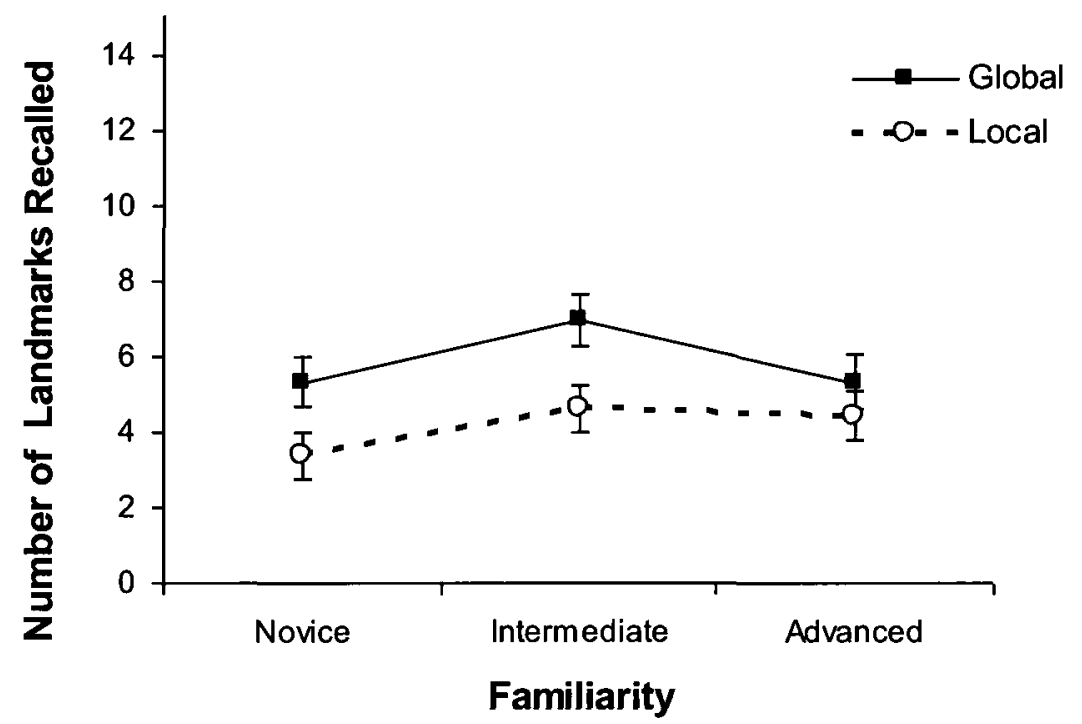

Figure 41. Number of landmarks recalled by type (+SE) across familiarity (groups).

A second analysis was conducted to investigate the number of landmarks as a function of type for the control group. Differences found within this group could be the 
result of either the effect of repeated testing, which vindicates the use of the current experimental design. Planned paired sample t-tests revealed a significantly larger number of global landmarks were recalled following the first block, $t(18)=-2.202, p<.05$. With increasing familiarity a statistically equal number of global and local landmarks were recalled (see Figure 42).

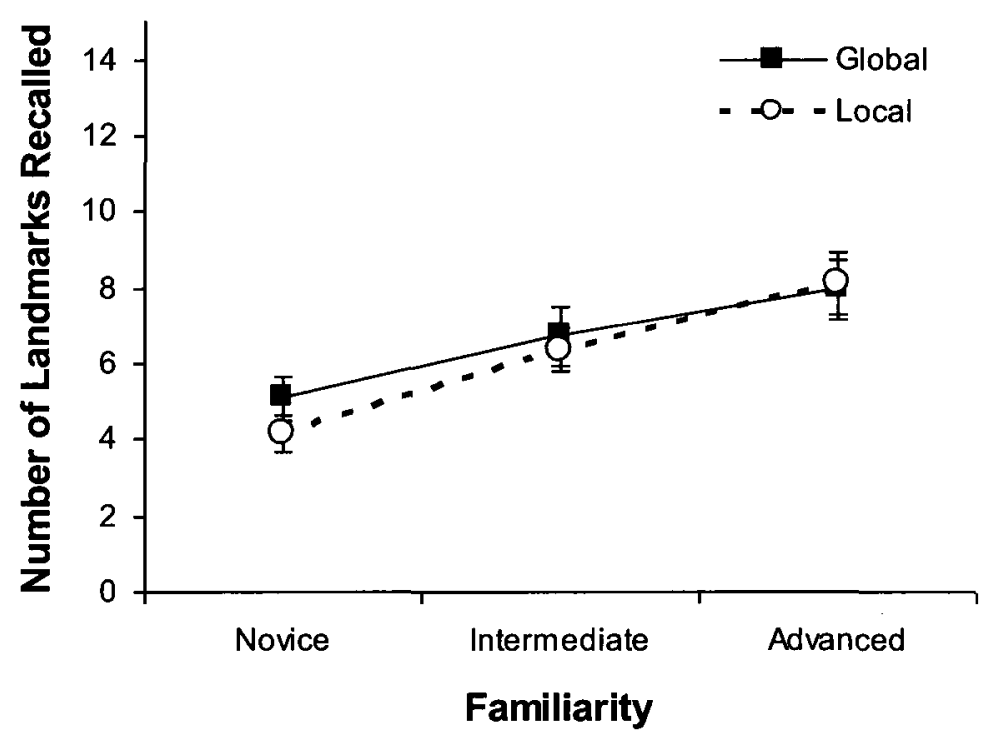

Figure 42. Number of landmarks recalled $(+\mathrm{SE})$ as a function of type across familiarity for the control group.

Landmarks included by location. Based on findings from Experiment 1 and 2, it was hypothesized that participants would recall more landmarks located in the periphery than internally, regardless of type. Planned paired sample t-tests were used to investigate differences between peripherally and internally located landmarks recalled within each group. It was found that significantly more peripheral landmarks were recalled at the 
novice, $t(18)=6.219, p<.001$, intermediate, $t(18)=9.114, p<.001$, and advanced, $t(18)$ $=6.922, p<.001$, levels of familiarity (see Figure 43 ). To test for significant differences across familiarity planned independent $t$-tests were used. It was found that significantly more peripheral landmarks were recalled by intermediates compared to novices, $t(18)=-$ $2.079, p<.05$. No differences in recall were found between levels of familiarity for landmarks located internally (see Figure 43).

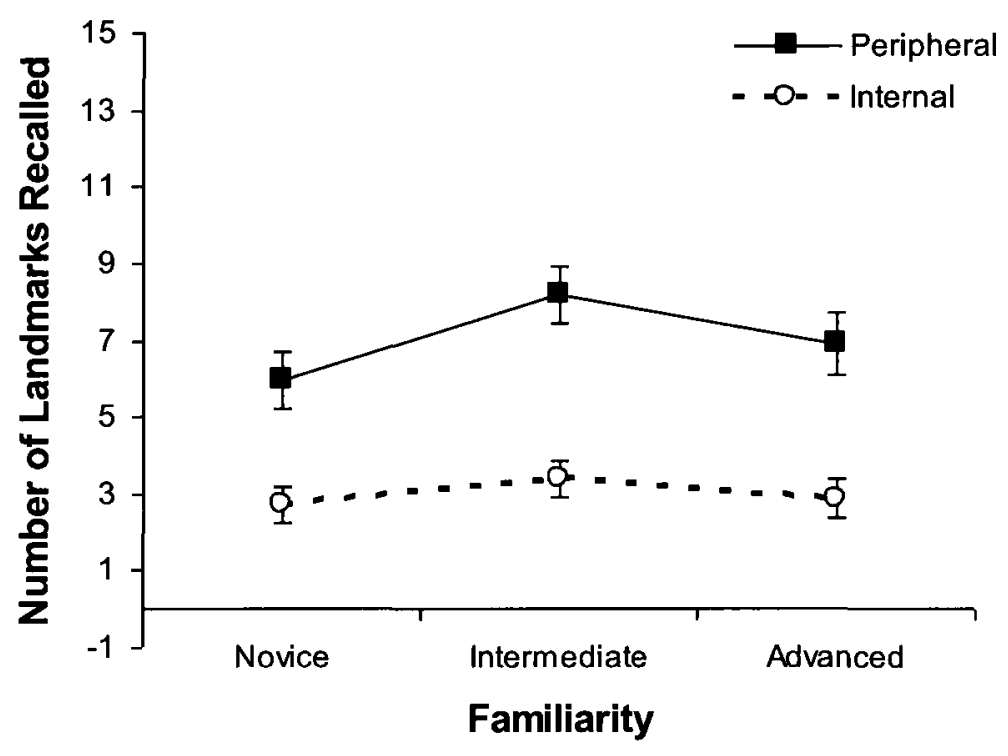

Figure 43.Number of landmarks recalled by location (+SE) across familiarity (groups).

A separate analysis was conducted to investigate the number of landmarks as a function of location for the control group. Paired sample t-tests revealed that this group recalled a significantly greater number of peripheral landmarks at the novice, $t(19)=$ $6.680, p<.001$, intermediate, $t(18)=5.942, p<.001$, and advanced, $t(19)=6.969, \mathrm{p}<$ .001 , levels of familiarity. The number of both peripheral, $t(19)=-4.943, p<.001$, and 
internal, $t(19)=-3.723, p=.001$, landmarks increased significantly between the novice and advanced familiarity (see Figure 44).

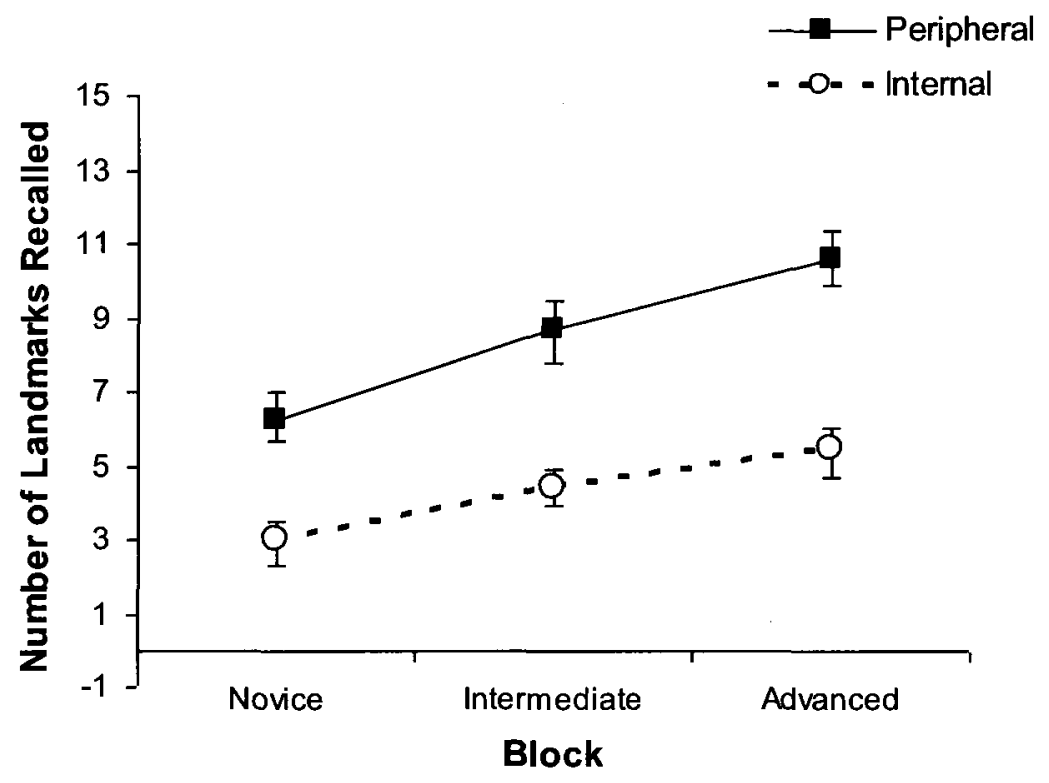

Figure 44. Number of landmarks recalled by location (+SE) across familiarity for the Control group.

Landmarks included by type and location. The role landmark type and location in combination may play in cognitive map formation was investigated in this analysis.

Based on the literature review, research questions, and Experiment 1 and 2 results, it was hypothesized that groups would recall a greater number of global peripheral landmarks when compared to all other types regardless of familiarity. A repeated measures ANOVA revealed significant 3-way interaction of landmark type, location and group, $F(2,55)=$ 4.220., $p<.05, \mu^{2}=.133$ (See Figure 45). This test also revealed significant 2-way interaction of landmark type by location, $F(1,55)=66.024, p<.001, \mu^{2}=.546$, and a 
marginal interaction between landmark location and group, $F(2,55)=2.937, p=.061$, $\mu^{2}=.096$. Significant main effects of landmark type, $F(1,55)=20.796, p=.000, \mu^{2}=.274$, and location $F(1,55)=161.207, p<.05, \mu^{2}=.746$, were also found. Post-hoc tests were used to investigate differences between the types and locations of landmarks. A significantly greater number of global landmarks were included in map drawings compared to local, $p=.000$, regardless of location. A significantly greater number of peripheral landmarks were included in map drawings, regardless of type, $p=.000$. Posthoc paired sample t-tests revealed that a significantly greater number of global peripheral landmarks were recalled compared to all other types regardless of familiarity level, $t(57)$ $=11.460, p<.001$ (see Figure 45), and similarly for the control group, $t(19)=6.443, p<$ .001 (and Figure 46).

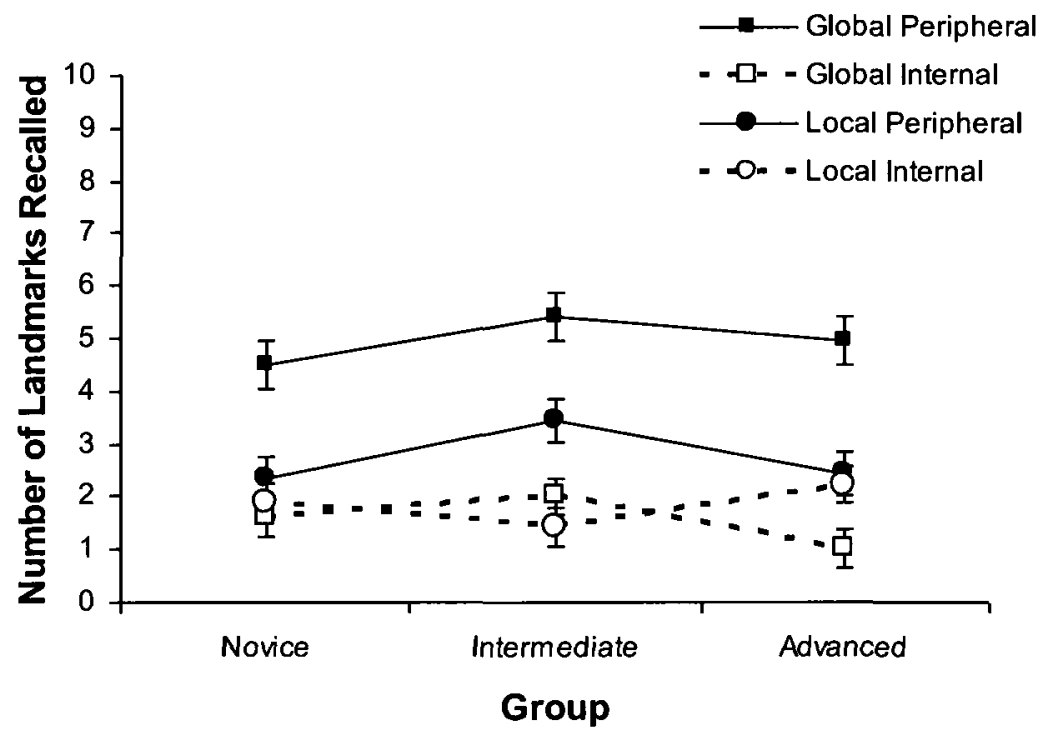

Figure 45. Number $(+\mathrm{SE})$ of landmarks by type and location included in participants sketch maps. 


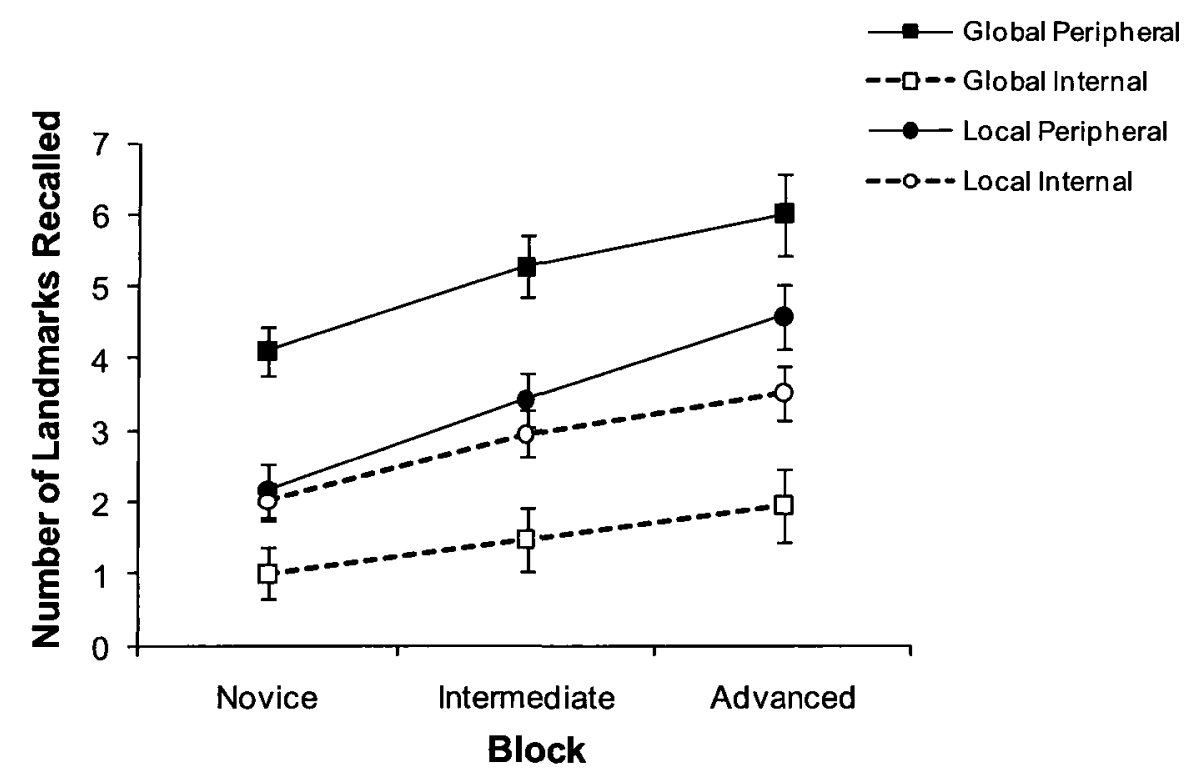

Figure 46. Number (+SE) of landmarks by type and location included in participants 1st map drawing for the control group.

\section{Relative Landmark Placement}

As in Experiment 1 and 2, relative landmark placement was used as an additional measure of sketch map accuracy and to provide a measure of differences in cognitive maps between groups. For each group, the five most frequently drawn landmarks were obtained (see Table 10) and a relative landmark placement ratio was then calculated for each map. This list was used to calculate the relative landmark placement ratio which was in turn used to compare cognitive maps between groups. The ratio was computed as follows:

Relative landmark placement ratio $=\#$ of correctly placed frequently drawn landmarks Total \# of frequently drawn landmarks $(=5)$ 
Table 10

The 5 Most Frequently Drawn Landmarks by Group

\begin{tabular}{|c|c|c|c|c|}
\hline & Experimental Groups & Frequency & Control Group & Frequency \\
\hline \multirow[t]{5}{*}{ Novice } & Bridge & 16 & Ship & 17 \\
\hline & Ship & 15 & Mountain & 14 \\
\hline & Mountain & 11 & Bridge & 14 \\
\hline & Stadium & 11 & Stadium & 14 \\
\hline & Cathedral & 10 & Cathedral & 10 \\
\hline \multirow[t]{5}{*}{ Intermediate } & Ship & 18 & Ship & 17 \\
\hline & Bridge & 16 & Heli-pad & 17 \\
\hline & Stadium & 15 & Bridge & 16 \\
\hline & Arch & 13 & Bus & 14 \\
\hline & Bus & 13 & Mountain & 14 \\
\hline \multirow[t]{5}{*}{ Advanced } & Ship & 17 & Ship & 19 \\
\hline & Bridge & 16 & Mountain & 18 \\
\hline & Mountain & 15 & Stadium & 18 \\
\hline & Stadium & 13 & Bridge & 16 \\
\hline & Arch & 11 & Heli-pad & 15 \\
\hline
\end{tabular}

Relative landmark placement as a function of familiarity. Planned independent ttests were used to investigate differences across experimental groups to assess the impact of familiarity on relative landmark placement. This analysis revealed no significant differences between levels of familiarity. A separate analysis was conducted for the 
control group included in their maps. Planned paired sample t-tests revealed that participants placed a significantly greater number of landmarks correctly within their maps between novice and intermediate levels of familiarity, $t(17)=-2.185, p<.05$ and novice and advanced levels, $t(19)=-4.359, p<.001$ (see Figure 47).

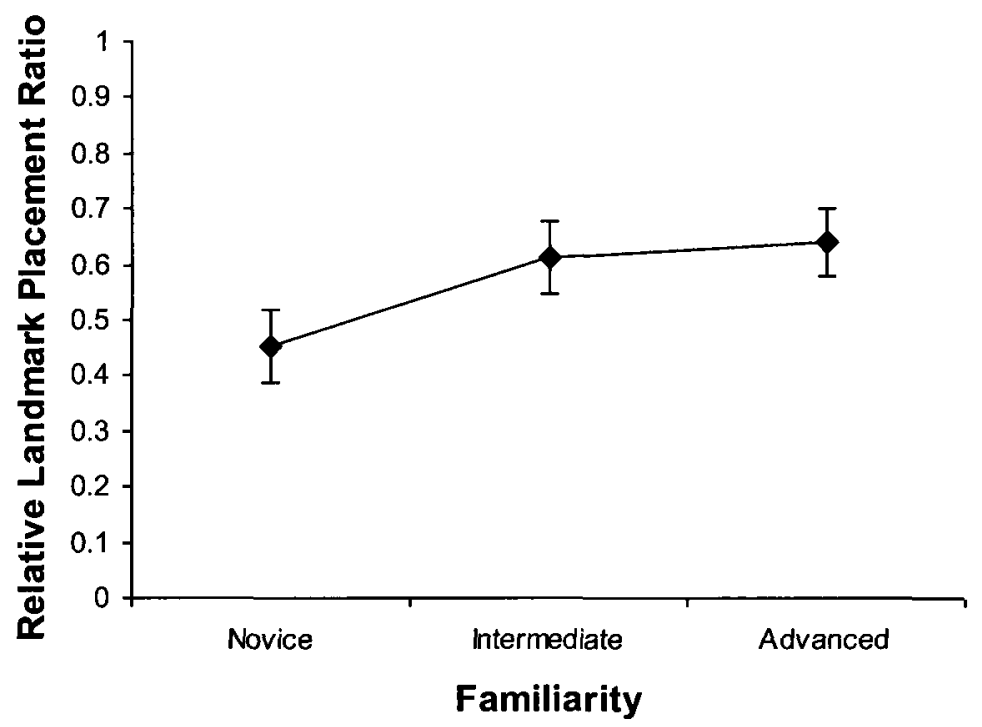

Figure 47. Relative landmark placement ratio (+SE) for control group.

Map goodness. Maps were rated by unbiased raters as a measure of "goodness" in order to investigate group differences among cognitive maps. The procedure used for rating maps can be found in Experiment 1. Prior to investigating map goodness between groups the degree of inter-rater reliability was calculated. Even though the map ratings are highly subjective the three raters had a significant intra-class correlation, indicating strong inter-rater reliability (.785). Means for each group and each map were computed and used to test map goodness ratings between experimental groups. A Kruskal-Wallis 
test was used to test for map rating differences between experimental groups, which were not found to differ. Wilcoxon rank sum tests were used to investigate differences between each block within the control group which revealed no significant map goodness differences between levels of familiarity.

In general, the sketch map analyses revealed that global landmarks, regardless of location, were recalled at a higher rate with differences between types dissipating as familiarity increased. Similarly, peripheral landmarks, regardless of type, were recalled at a higher rate across all levels of familiarity when compared to internal ones. When the combination of landmark type and location was investigated, global landmarks located in the periphery were recalled at a higher rate when compared against all other landmarks, regardless of amount of familiarity.

\section{Spatial Strategy Questionnaire}

The mean number of true and false answers to each question for each experimental group was calculated. Tests were used to investigate the proportion of true to false responses for each question within each group. To investigate differences between groups, the mean number of times each group responded "true" versus "false" was analyzed for each question separately. To analyze differences between groups, the mean ratio of "true" to "false" answers was analyzed for each question separately. A Kruskal-Wallis test was used to test for differences between groups, which did not exist. Chi-square tests were used to investigate differences for each question within each group (see Table 11). These tests revealed that regardless of group, the majority of participants kept track of the direction in which they were going, kept track of where they were in 
relation to a reference point such as the centre, water, mountain or other object, visualized a map or layout of the area, and felt that objects were helpful to section the world or find different areas within the world.

Table 11

Spatial Strategy Questions: Proportion of True to False Questions for Groups.

\begin{tabular}{|c|c|c|c|}
\hline \multirow[b]{2}{*}{ Question } & \multicolumn{3}{|c|}{$\begin{array}{l}\text { Frequency of True Answers } \\
\text { Frequency of False Answers }\end{array}$} \\
\hline & Novice & Intermediate & Advanced \\
\hline $\begin{array}{l}\text { I kept track of the direction in which I was } \\
\text { going. }\end{array}$ & $\frac{15}{6}^{*}$ & $\frac{15}{5}^{*}$ & $\frac{14}{5}^{*}$ \\
\hline $\begin{array}{l}\text { I always kept in mind the point from which I } \\
\text { had entered the world. }\end{array}$ & $\frac{5}{16}^{*}$ & $\frac{7}{13}$ & $\frac{9}{10}$ \\
\hline $\begin{array}{l}\text { I thought of my location in the world in } \\
\text { terms of North, South, East and West. }\end{array}$ & $\frac{0}{21}^{* *}$ & $\frac{2}{18}^{* *}$ & $\frac{3}{16}^{* *}$ \\
\hline $\begin{array}{l}\text { Whenever I made a turn I knew which } \\
\text { direction I was facing. }\end{array}$ & $\frac{8}{13}$ & $\frac{12}{8}$ & $\frac{10}{9}$ \\
\hline $\begin{array}{l}\text { I kept track of where I was in relation to a } \\
\text { reference point such as the centre, water, }\end{array}$ & $\frac{18}{3}^{* *}$ & $\frac{18}{2}^{* *}$ & $\frac{16}{3}^{*}$ \\
\hline mountain etc. & & & \\
\hline $\begin{array}{l}\text { As I went I made a mental note of the } \\
\text { distance I traveled. }\end{array}$ & $\frac{8}{13}$ & $\frac{5}{15}^{*}$ & $\frac{7}{12}$ \\
\hline I visualized a map or layout of the area in & $\frac{19}{2}^{* *}$ & $\frac{19}{1}^{* *}$ & $\frac{19}{0}^{* *}$ \\
\hline my mind as I went. & & & \\
\hline Objects were helpful to section the world or & $\frac{20}{1}^{* *}$ & $\frac{19}{1}^{* *}$ & $\frac{17}{2}^{* *}$ \\
\hline
\end{tabular}


find different areas within the world.

I counted the number of roads I passed to

$\frac{6}{15}$

$\frac{8}{12}$

$\frac{7}{12}$

help me find my way.

$* * \mathrm{p}<.001, * \mathrm{p}<.05$

Way-finding Decision Task (WDT) Performance

Making correct way-finding decisions can reflect the level of acquired route knowledge. The WDT required participants to choose an action required to reach a specific landmark along a route. This analysis included WDT performance for landmarks by their type, location, and combinations of type and location as a function of familiarity between experimental groups and within the control. The mean proportion of correct responses was used to test for differences in all WDT analyses. The descriptive statistics associated with the WDT analyses for Experiment 3 can be found in Appendix U.

WDT performance as a function of landmark type. Literature states that local landmarks have a positive impact on route knowledge acquisition (Aginsky, et al., 1997; Parush \& Berman, 2004; Tlauka \& Wilson, 1994). This may still be the case, though the first two experiments showed that the amount of familiarity impacts the type of landmark that aids way-finding. Specifically performance was found to be better by way of global landmarks while unfamiliar with the environment and local as familiarity increases. If findings in this analysis support the literature, questions referring to local landmarks will result in better performance compared to global landmarks, regardless of familiarity. However if findings from Experiments 1 and 2 are supported then questions referring to global landmarks will result in better performance compared to local landmarks with little 
familiarity. As familiarity increases so will performance for questions referring to local landmarks.

A combination of planned comparison paired-sample and independent t-tests were performed to test for differences of WDT performance within and across the three levels of familiarity (groups). This analysis revealed what types of landmark play a supportive role in way-finding decisions as a function of familiarity. No differences were found between landmark types at any of the levels of familiarity, as revealed by paired sample ttests. Performance in making way-finding decisions referring to global, $t(29.97)=-2.462$, $p<.05$, and local, $t(36)=-3.723, p<.001$, landmarks both significantly improved between novice and intermediate (see Figure 48). No other differences were found.

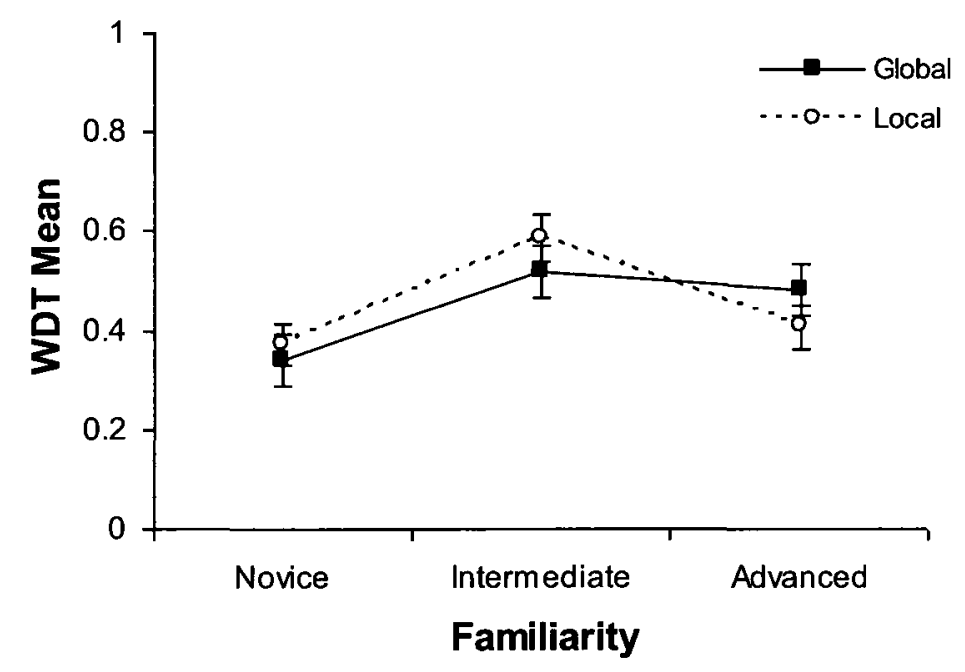

Figure 48. WDT performance (+SE) by landmark type across level of familiarity. 
A separate analysis was conducted to investigate the same hypotheses for the control group. Planned paired sample t-tests revealed that there was significant improvement in performance relating to local landmarks between novice and intermediate levels of familiarity, $t(18)=-2.802, p<.05$. Participants performed significantly better when WDT questions referred to local landmarks compared to global with advanced familiarity, $t(19)=-2.116, \mathrm{p}<.05$ (see Figure 49).

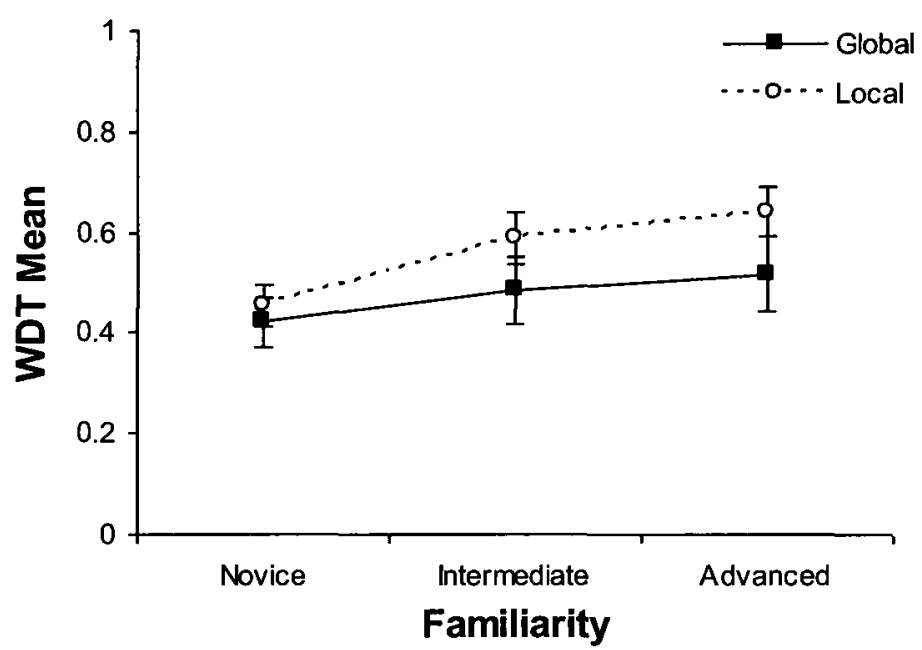

Figure 49. WDT performance $(+\mathrm{SE})$ by landmark type across level of familiarity for the control group.

WDT performance as a function of landmark location. Planned comparison paired sample and independent $t$-tests were used to analyze differences in WDT performance within and across the three levels of familiarity for landmark location. This analysis provided insight regarding differences between peripheral and internal landmark 
acquisition in reference way-finding decisions made at and between each level of familiarity.

Given the previous findings in Experiment 1 and 2 I hypothesized that performance for peripheral and internal landmark WDT questions would not differ. No differences were found between landmark locations within each familiarity level.

Independent sample t-tests revealed a significant increase in performance between novice and intermediate levels of familiarity when making way-finding decisions in reference to peripheral, $t(37)=-2.297, p<.05$, and internal, $t(37)=-2.541, p<.05$, landmarks (see Figure 50).

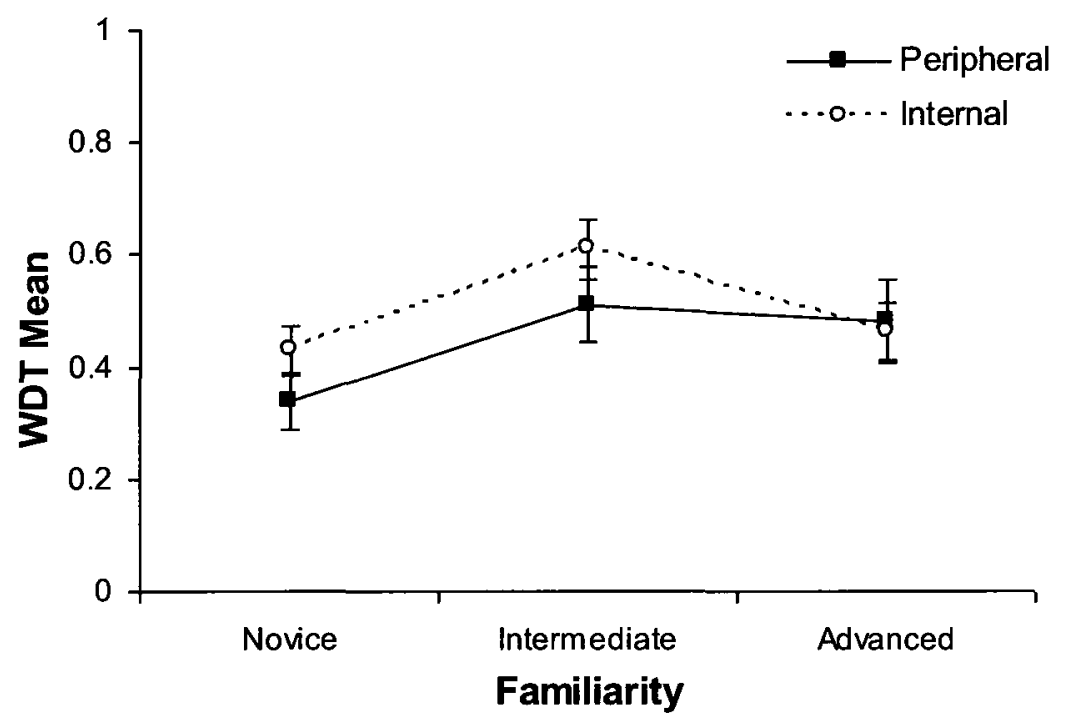

Figure 50. WDT performance (+SE) by landmark location across level of familiarity.

A separate analysis was conducted to investigate results of the control group. Planned paired sample t-tests revealed no significant differences within each landmark 
location across familiarity, nor were there any differences between landmarks' locations within each block.

WDT performance as a function of landmark location and type. The role that the combination of landmark type and location play in making way-finding decisions was investigated in this analysis. Based on the literature review, research questions, and Experiment 1 and 2 results, I hypothesized that performance would be better for WDT referring to local landmarks when compared to all other types regardless of familiarity. Repeated measures ANOVA revealed no significant differences between or within experimental groups.

A separate repeated measures ANOVA was conducted to investigate differences between landmarks type - location for the control group across familiarity. It was found that performance significantly improved across familiarity, regardless of landmark typelocation, $F(2,17)=5.445, p<.05, \mu^{2}=.133$ (See Figure 51). Post-hoc tests revealed a significant improvement between the novice and advanced levels of familiarity. It was also found that regardless of location or familiarity performance in reference to local landmarks was significantly better than global overall, $F(1,18)=4.475, p<.05, \mu^{2}=$ .200 . 


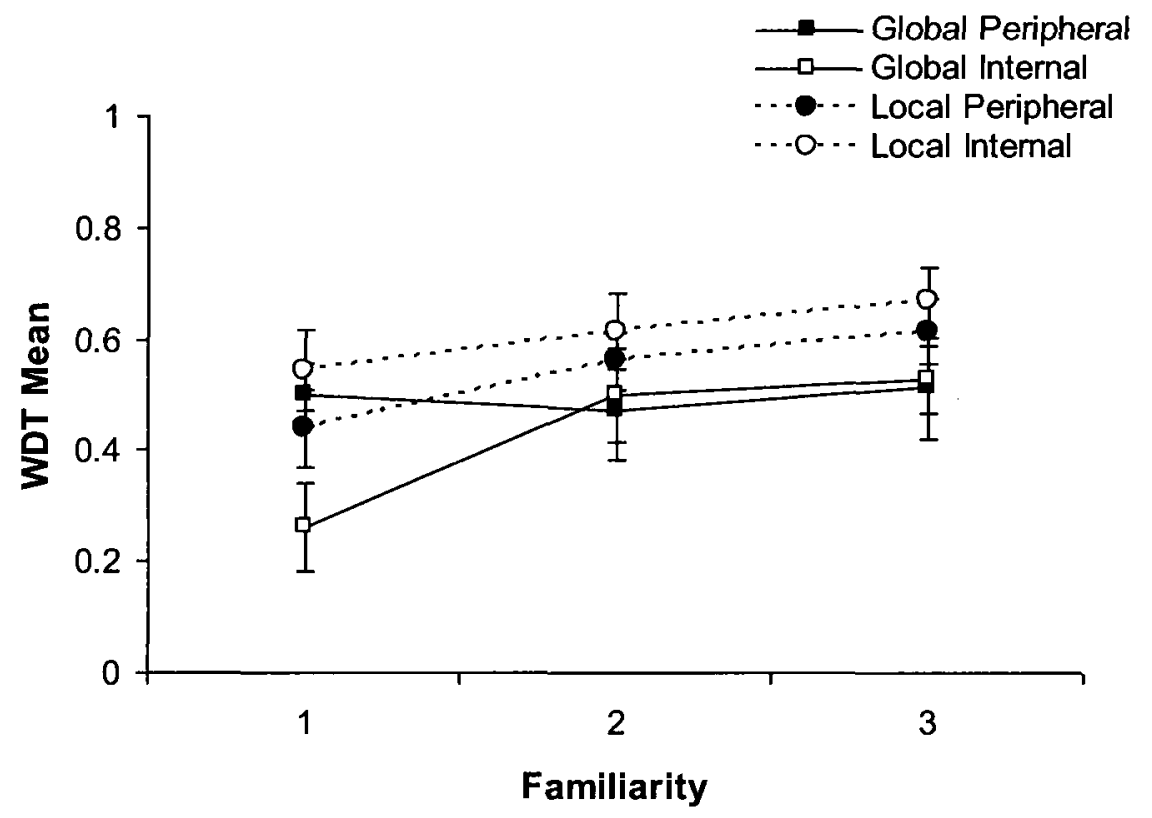

Figure 51. WDT performance by landmark type and location across familiarity for the control group.

Overall, findings from this analysis were not consistent for those in the experimental groups compared to the control. In general WDT performance improved for both types in the experimental groups. However, the control groups' performance for local landmarks within WDT was found to improve significantly and was also significantly better when compared to global. Differences found between the experimental groups and control group in this analysis is difficult to explain. The differences could be due either to the effect of repeated testing on the control group, or the advanced groups' poor performance may be due to fatigue. The data was not sufficient to provide an assertive explanation. No preference for landmark location was found across familiarity for WDT performance. 


\section{Experiment 3: Discussion}

The first two experiments demonstrated temporal precedence for global landmarks during way-finding; however, the paradigms used did not afford the investigation of temporal precedence during spatial knowledge acquisition. Therefore, Experiment 3 focused on the combined impact of landmark type and location within the spatial knowledge acquisition process. Spatial memory tasks were given to participants at different levels of familiarity during their way-finding to capture differences across the acquisition process. If precedence exists across familiarity for specific types and locations of landmarks, differences between the three experimental groups' spatial memory performance would have reflected this.

The sketch map findings provided insight into the roles that type and location of landmarks play within the development of survey knowledge acquisition. First, findings suggest that the acquisition and recall of global landmarks is superior to that of local ones early on in the process. As development progresses, differences between landmark acquisition and recall based on the type diminishes. Interestingly, the same cannot be said for landmarks based on location. The superior role that landmarks in the periphery play does not dissipate with the development of spatial knowledge as does the type, indicating a unique role for landmarks by their location for use in survey knowledge recall across familiarity.

The roles landmarks play by their type and location, in terms of making wayfinding decisions, appear to change across level of familiarity. Performance for the WDT was significantly better for the control group when the way-finding questions referred to 
local versus global landmarks. Although this finding could have in part been a result of the repeated testing, it provides further confirmation for the argument that the role of local landmarks consists of information that aids way-finding decisions. Further, when differences between types were analyzed, preference for local landmark use was only found during the more advanced phase of learning, providing further support for the claim that local landmarks provide information to make way-finding decisions only at greater levels of familiarity.

The different results found between spatial knowledge acquisition and wayfinding decisions suggest that the sensory and spatial information for landmarks may be interconnected in that their impact by the combined type and location will depend on the spatial task. That is, for way-finding there is a preference to rely on type independent of location, whereas for spatial knowledge type is dependent on location in combination. This finding indicates interconnection between the two types of information associated with landmarks, the sensory and spatial, which may be dependent on the spatial task at hand.

A primary aim of this final experiment was to shed light onto the process by which spatial knowledge is acquired. Again, there are two commonly discussed theoretical approaches that account for the acquisition of spatial knowledge. The first suggests that the details or landmarks are initially learned followed by the global features in a stage-like fashion (Siegel \& White, 1975; Yeap \& Jeffries, 2000), whereas others' suggest that cognitive map formation is parallel in nature (Aginsky et al., 1997; Lynch, 1960; MacEachren, 1992; Montello, 1998; Peponis, Zimring, \& Choi 1990). Findings 
suggest that there may not be a clear-cut answer supporting any particular theory. In fact, both may be in the right context. For example, the LRS model never states the difference between the types of landmarks. Although there seems to be a difference in terms of the acquisition between types and locations of landmarks, whether or not this is then followed by route and survey knowledge was not demonstrated. Further, when global landmarks are available they are acquired earlier than local, which may facilitate the acquisition of survey knowledge earlier than if they were not available. Findings from this study suggest that the presence of global landmarks may in fact act as an aid compared to when they are not provided. As global landmarks seem to be acquired earlier, some level of survey knowledge may be acquired prior to or in parallel to the details provided by local landmarks. Again, this is suggested as global landmarks are assumed to be associated with more survey related knowledge such as direction and distance estimation and aid in organizing and providing structure to the environment.

Overall, Experiment 3 provides support for three claims. First, the roles landmarks play by their type and location, in terms of making way-finding decisions, changes across familiarity with precedence for local landmarks increasing with familiarity. Second, the acquisition and recall of global landmarks is superior to that of local early in the development of spatial knowledge acquisition with the differences dissipating across familiarity. Conversely the recall of peripheral landmarks does not dissipate with increasing familiarity. Third, an LRS stage-like process of spatial knowledge acquisition was not supported. In fact, results support a more parallel, top- 
down process as global - peripheral landmarks intended to provide structure and higher level spatial information are recalled at a better rate regardless of familiarity. 


\section{General Discussion}

Landmarks can be one of the most useful navigational tools for finding our way through an environment. Ironically, the literature has been deficient in addressing the specific features of landmarks and their associated significance for way-finding and spatial knowledge acquisition. As the production and use of 3D VE's and navigation aids increase, a better understanding of the impact of landmarks based on their features and how we use them to find our way is needed. Practically, understanding the impact of landmarks will enable better design of electronic spaces and aids. Theoretically, this understanding will add to the body of literature that lacks in the cognitive impact of landmarks by their features.

The literature reviewed suggests an entanglement of two primary landmark features, their type (global - local) and their location (peripheral - internal). In the current research, three experiments were conducted to investigate the isolated and combined impact of landmarks by their type and location on way-finding and spatial memory tasks. Of specific interest was whether landmarks based on their type and location have equivalent impact within way-finding and spatial knowledge development. The research strategy involved creating and placing landmarks within a 3D virtual environment. These landmarks were classified as either global or local, following common definitions from the literature, and located either in the periphery or the internal part of the VE (landmark location). Experiment 1 involved isolating the impact of landmarks by their type (global versus local) independent of their location on way-finding and spatial knowledge. Experiment 2 focused directly on the impact of landmark location (peripheral and 
internal). Experiment 3 focused on the impact of landmark type and location as a function of experience with the additional aim of contributing to current theories of spatial knowledge acquisition. In this chapter I summarize the key findings from the current studies and propose a general theoretical framework to account for landmark impact on spatial cognition, while taking in account related literature.

\section{Key Findings and Related Literature}

In sum, as the spatial strategy results indicated, regardless of the type of location of landmarks they were helpful to keep track of direction and of to aid in helping keep track of where one is. When unfamiliar with the VE, global landmarks had a positive impact on way-finding. As familiarity increased, way-finding performance improved in the presence of local but not global landmarks. This finding was persistent regardless of whether the global and local landmarks were located in the periphery, internally, or both. In other words, way-finding performance was associated with landmark type independent of location. In contrast to way-finding, it was found that spatial knowledge, reflected in the recall of the VE configuration and landmarks, was dependent on the location of landmarks, particularly when located in the periphery. Therefore, the key findings imply a distinction between landmark acquisition and use as a function of their type and location. How and when landmarks are relied upon as a function of their type and location is determined by amount of familiarity with the environment and the spatial task being considered.

Understanding parallel fields of research that investigate similar processes may provide further explanations from different perspectives. Specifically, comparative 
cognition and neuropsychology also examine the impact of environmental features on visual processing and behavior. This body of literature provides some insight into why the various combinations of landmarks by type and location are found. It is not my intention to provide an in-depth review of this body of research but the similarities should be mentioned. Two related aspects within this body of literature are discussed; specifically, neural processes sensitive to and influenced by different environmental features. Further, as findings within this thesis were found to be dependent on the spatial task a review of how the neural processes may be influenced by these spatial tasks is provided.

First, Burgess (2008) proposed a neural model of spatial processing suggesting that two separate neural systems process two different aspects of environmental information. Burgess claims that cells within the hippocampal system are responsible for processing boundary related information, and the dorsal striatal system is responsible for processing fine-grained cues or local landmark information (2008). He suggests that the extent of and proximity to a "boundary" can influence hippocampal processing and likewise environmental stimuli that correspond to "landmarks" would be reflected in their influence on striatal processing. Similar ideas were independently proposed by Jacobs (2003; 2003a), a comparative psychologist. She suggests that "parallel", dissociable cognitive maps may exist, which play different roles and are sensitive to different environmental features (Jacobs, 2003). Jacobs refers to one map as the 'bearing' map which is based on large scale directional information, and a 'local or sketch' map which is sensitive to fine grained local cues. Jacobs (2003) suggested that each map plays a 
different role and may be sensitive to very different aspects of the environment. However, with experience maps may become integrated with each other.

Second, Milner \& Goodale (1995) discovered two distinct but interacting visual processing streams, referred to as the 'dorsal' and 'ventral' pathways. Goodale (1994) defined these two systems by way of the manner in which the visual information is used and transformed to achieve different goals. The associated systems are referred to as the 'perception-action' system and the 'cognitive' system (Creem \& Proffitt, 1998, 2001; Liben, 1988, 1999). As these neural processes are mediated by different 'frames of reference' it is important to touch upon them prior to the discussion. A frame of reference is a means of representing the locations of entities in space (Klatzky, 1998). When taking an egocentric frame of reference spatial locations are coded by referring to our own viewpoint. Egocentric coding is dynamic and needs continuous updating as we move (Committeri et al., 2004). When taking an allocentric reference frame we code space relative to points within a framework external to ourselves and independent of our position (Klatzky, 1998).

The perception-action system (also known as the dorsal stream or the "how" system) visually guides actions, processes the location of objects, and is used for tasks that require constant updating from an egocentric frame of reference, for example wayfinding or grasping objects (Neggers, Van der Lubbe, Ramsey, \& Postma, 2006). The cognitive system (also known as the ventral stream or the "what" pathway) is used to process information required to identify objects, is memory driven, and is used for allocentric tasks such as object recognition (Milner \& Goodale, 1995). These related 
findings provide insight into the differing results found in the current thesis in terms of the roles landmarks play by their type and location between way-finding (perceptionaction) and spatial knowledge recall (cognitive).

In summary, two concepts relevant to this thesis can be adopted from the comparative and neuropsychological research reviewed. First, there are two neural processes that are sensitive to various environmental features; one process is sensitive to boundary related information and the other to fine-grained cues. It can be suggested that these two processes may be mediated by landmarks by their type and location. The hippocampal system may be sensitive to global landmarks located in the periphery whereas the striatal system may be sensitive to local landmarks. As these studies have not controlled landmarks by their type and location, to my knowledge, it is difficult to suggest what the exact impact landmarks by their specific types and locations may have on the two neural processes. Second, the neural distinction between how information is processed based on the type of task, perception-action (way-finding) and cognitive (spatial knowledge recall), speaks perhaps to why the features of landmarks impacted the way-finding and spatial knowledge tasks different ways. This also may speak to the frame of reference taken for each type of task. Specifically, that various environmental features have a behavioural and neural impact and that their impact may be dependent on the frame of reference taken (or spatial task).

\section{Framework: Dual Process of Landmark Knowledge}

Although theories of spatial knowledge acquisition address landmarks in a broad sense, they do not account for the diverse impact landmarks could have by their various 
features. This dissertation offers a theoretical framework to address this gap. Additionally, this framework attempts to amalgamate the findings with existing theories of spatial cognition to explain how and why landmarks by their type and locations may be used to way-find and integrated into our cognitive maps. The framework is aimed to contribute to current theories, enable researchers to predict the outcome of future experiments when investigating landmarks, and guide future VE development to aid wayfinding.

The central principle of this framework claims that there are two distinct processes defined by the type of information that each is sensitive to; one process relies on the sensory features of landmarks and one on the spatial. Additionally, it is suggested that these two processes are compensatory and interconnected. This framework is supported by the current findings as well as related literature and the central principle and associated compensatory and interconnectedness characteristics are discussed in detail next.

\section{Central Principle: Dual Process}

Landmark knowledge acquisition relies heavily on sensory information. In this thesis, the sensory information includes the visual aspects of landmarks; that is, their size and associated visibility. The proposed sensory process is strongly related to the active exploration and search tasks. Within the sensory process, people initially attend to the more visible landmarks, regardless of their location in the environment (see Table 12). It is argued that this provides the initial orientation or bearing framework needed to acquire in an unfamiliar environment. As the environment becomes more familiar, more attention 
is paid to local landmarks, which provides the information required to make local wayfinding decisions. Conversely, the proposed 'spatial' process is strongly related to the encoding and retrieval of spatial information from memory. This is primarily expressed in spatial memory tasks where as there was dominance for landmarks located in the periphery of the environment as opposed to landmarks located in the internal parts of the environment (see Table 12). This dominance was consistent regardless of global or local.

In relation to the supporting neural research discussed it can be suggested that the spatial and sensory processes proposed in this framework may be tapping into or reflect the two processes found within the neuropsychology literature. It is suggested that the sensory and spatial processes may not directly map onto the two visual processing streams they may influence each in predictable ways. Although further research is required, predictions can be made regarding how the specific sensory and spatial processes interact and influence with the neural pathways. For example, the sensory process may influence the "perception-action system" more than the spatial process as it was strongly related to active way-finding. The two defining characteristics of the dual process are discussed next.

Compensatory Nature of the Processes. Information related to the sensory and spatial processes is acquired and processed in a compensatory manner. Specifically, when only partial sensory or spatial information is available, the partial information seems to be sufficient to way-find and acquire survey knowledge (see Table 12). Although acquiring specific types of spatial knowledge and tasks may be easier with certain types and locations of landmarks the compensatory nature provides the ability to acquire spatial 
knowledge if the environmental information of a specific type or location of landmark is not available.

The Interconnectedness of the Processes. The processes are said to be interconnected as they can be thought of as two parts of a system that interact with and rely on one another. The spatial and sensory processes are not mutually exclusive and work simultaneously in ways that depend on the spatial task. The sensory process was relied on during way-finding whereas a combination of sensory and spatial processes was processed during spatial knowledge recall. It is not suggested that at any time only one process alone is occurring, as a landmark within an environment always consists of both a location and a sensory. The sensory and spatial processes act together to provide a specific purpose in terms of the spatial task being executed (see Table 12).

Table 12

Elements of Framework and Associated Results

Framework Support from Results

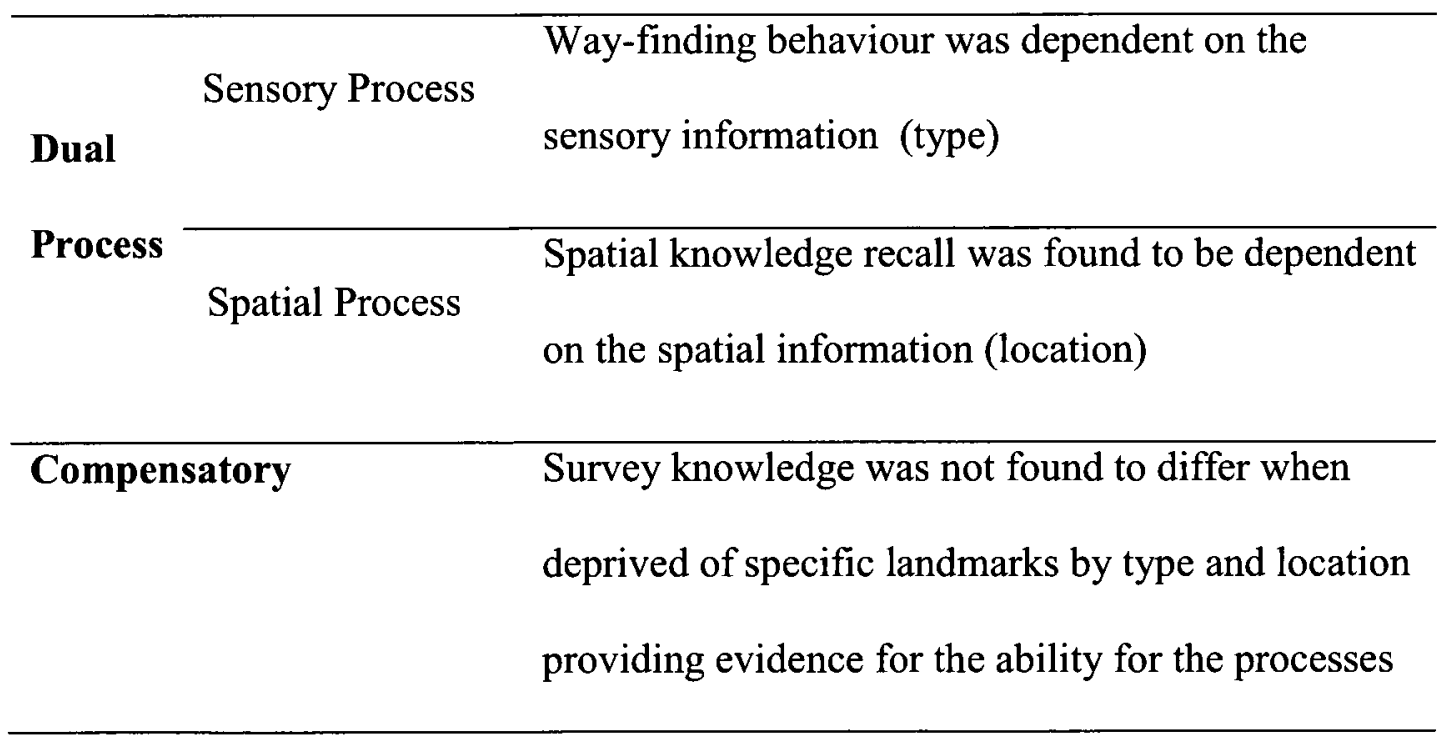


associated with acquiring and recalling landmarks

to compensate for one another within each process.

Interconnected

When recalling spatial knowledge there is a

dependency on the interaction between the sensory

and the spatial processes.

Framework Evaluation and Predictions. Several hypothetical situations and thoughts are provided to assess this framework. First, manipulating the sensory and spatial information to the "extreme" would provide further evidence as to how these two processes are interconnected and dependent on the spatial task. If the findings were dependent solely on the two processes proposed, then creating and testing landmarks that were identical except for their location and type would provide further insight into their impact. For example, if a VE was used to test this framework that contained identical landmarks but were manipulated based only on their size and visibility (global and local) and their location (peripheral and internal) would the findings be replicated? If this framework holds true then the findings should be replicated and perhaps be more robust. However, this is based on an assumption that the sensory and spatial information is the only information that is relied upon to utilize landmarks to way-find by. One important definition of a landmark is that each is distinctive enough from the others that it can provide information separate from other objects and landmarks in the environment (Vinson, 1999). Therefore, if there was a way to hold the spatial and sensory information 
constant across landmarks by their type and location, while at the same time providing information to way-find that is independent of landmarks, this could provide a definitive test of this proposed framework.

Second, the elements of this framework are dependent on findings of a 'relatively' short experience, compared to the familiarity we have with our own neighborhoods. Therefore, what would the framework predict if the amount of time spent in the world was extended? With even more familiarity than what was tested in the current series of studies can we expect that the sensory process is still dominant while way-finding and independent of the spatial? If this is the case, then the differences found between the global and local impact on way-finding would increase all the more. That is that the impact of local landmarks would increase to an asymptote higher than that of global landmarks.

Finally predictions can be made regarding how landmarks by their spatial and sensory information may impact navigation tools and vice versa. Suppose two groups were investigated; one is provided with a map indicating where the global landmarks are and one group with a map indicating local landmarks. According to this framework those with the global-map would perform way-finding tasks more efficiently while unfamiliar with the testing environment. As familiarity increases the performance for the group with the local - map would perform more efficiently than the global-map group.

\section{Implications for Theories of Spatial Knowledge Acquisition}

This section provides an overview of implications that the current findings has for theories of spatial knowledge acquisition and global - precedence theory of attention. 
The theoretical goal of this dissertation was to provide insight into the impact, roles, and processes used to acquire spatial knowledge based on landmark size and location.

It has been argued that the acquisition of spatial information occurs in a consecutive stage-like fashion, beginning with landmarks, followed by route, and finally survey knowledge (Siegel \& White, 1975). Other theories suggest that global properties, or survey knowledge, can be acquired earlier in the process, followed by the details or in parallel with the details and other spatial information (Hunt \& Waller, 1999; Montello, 1998; Parush \& Berman, 2004). The order in which global and local landmarks are acquired can provide insight into how spatial knowledge is processed. As global landmarks seemed to be attended to prior to local and recalled at a better rate, it is suggested that some level of survey knowledge is acquired prior to or in parallel with the details (Couclelis, et al., 1987; Golledge, 1992; Hirtle \& Jonides, 1985; Hirtle \& Kallman, 1988; Newman et al., 2007).

The results from this thesis suggest that there may not be a straightforward answer as to which theory is supported; in fact both may be applied in the relevant context. The LRS model does not distinguish between the different types of landmarks and their impact on the model. The current findings can provide an additional level of detail to the LRS model as it was found that there is a difference between the acquisition of landmarks based on their types and locations. Conversely, the current findings can also provide an argument against this model. When global landmarks are available they are acquired earlier than local. As global landmarks are assumed to be related to information that provides a higher level of knowledge (e.g., distance, direction, frame of reference) it can 
be argued that the acquisition of a higher level of spatial knowledge prior to route knowledge can occur earlier than what the LRS model would predict.

Previous research suggests that the LRS model does not hold entirely true in the presence of aids. It has been found that acquiring survey knowledge by way of a map, referred to as secondary survey knowledge, is not as complete as survey knowledge acquired by way of first person in-world interaction, referred to as primary survey knowledge (Thorndyke \& Hayes-Roth, 1982). It could be suggested that the presence of global landmarks may act as an aid by initially providing quick and "simple" survey knowledge similar to that of a map. As global landmarks were acquired earlier than local, some level of survey knowledge may be acquired prior to or in parallel to the details provided by local landmarks. As suggested earlier the LRS model may be irrelevant depending on the context. The presence of global landmarks may be one of the situations where the LRS model does not hold true. This is argued because of the way in which spatial information is acquired in the presence of global landmarks supports a more "parallel" or top-down type of spatial knowledge acquisition process.

Findings within the global precedence literature of perception and attention has implications as to what features are visually attended to within a 3D VE and the strategy used to acquire and recall information about a visual scene (Davidoff, Fonteneau, \& Fagot, 2008; Navon, 1983, 1991, 2003; Navon \& Norman, 1983). In general, this literature provided two theoretical focal points that related to this dissertation. First, the processing strategies used within acquiring spatial knowledge, specifically that a similar strategy of information processing may be applied to processing global and local 
landmarks. It is argued that visual scenes are decomposed rather than built up. The findings within global precedence literature turned out to be comparable to that found in the current studies. It is suggested that the global landmarks were attended to prior to the local and that this provides further support that when the appropriate spatial information is available (i.e., global landmarks or other aids) the processing strategy is to encode the "bigger picture" or structure and then layer the details within this in a top-down way. Therefore, there seems to be some support for a similar information processing strategy used to attend to features within a visual scene and for 3D environments.

\section{Generality and Practical Implications}

The design of the VE used can impact the results. This is always a concern in terms of how transferrable the results with the use of a VE will be across all types of environments. In this thesis, a preference for landmarks located in the periphery was found during spatial knowledge recall. This can be argued as an artifact of the layout of the VE as there was a distinctive "boundary" or periphery which may have in some way biased users to attend to the landmarks situated in the periphery. This finding is interesting regardless, as most virtual worlds are designed with a distinct boundary and therefore these findings may generalize nicely to most virtual environments. Further, as stated in the literature, way-finders may be neurally and behaviourally predisposed to attend to landmarks situated near the boundary or periphery in order to provide bearing information that is incorporated into their cognitive maps by way of the hippocampal system which is responsible for processing boundary related information. Therefore, even if the world did not have a pre-designed boundary it could be predicted that participants 
may create their own with landmarks or other environmental structures provided. That said, it would be interesting to see if the precedence for peripheral landmarks is generalizable to a design that does not contain a distinct boundary. In an attempt to be as ecologically valid as possible the environment was created as an open "realistic" city while controlling for the size of landmarks and the location of most. Although this world contained each type of landmark and location, that is, global and local landmarks each located in the periphery and internally, the numbers were not entirely equal between the two locations. Even so, every attempt was made to control for the unequal numbers of each type of landmark at each location by comparing "ratios" and not means when involving landmark location comparisons. Given that the results were found based on ratios it is argued that these results will be consistent if the study were run again with number of landmarks by their location equalized.

The global and local landmarks were created based on a working definition of each which was based on visual saliency. It can be argued that although this was the appropriate preliminary step for an empirical investigation of global and local landmarks, future investigations can benefit from more concise definitions. I argue that one direction the term can take is to define landmarks along a continuum. As size is a continuous variable, global and local landmarks can have a degree of "global-ness" and/or "localness". And that the level of impact on their specific uses (way-finding and spatial knowledge recall) can be a directly related to its amount of their global-ness and localness. As an example, the larger and more useful for providing compass directions the landmark is (both measurable variables) the more it can be considered a "global" 
landmark. The closer and more helpful the landmark is to a target to be found (also measurable variables) the more "local" it is. This would provide a more concise working definition of global and local landmarks.

For the purpose of this research a global landmark was defined as an object that is visible from a large area in the environment, provide a frame of reference or compasslike information, help organize and provide structure to the environment, and do not change visually in a significant way when the observer moves a small distance. Local landmarks are described as objects that are visible within a localized area and used at specific points while making way-finding decisions (Glenn \& Chignell, 1992, Nielsen, 1995; Ruddle, Payne \& Jones, 1997; Steck \& Mallot, 2002). It could be argued that not all global and local landmarks were used as such by participants and therefore could have created "noise" within the data. Future research should implement a more rigorously controlled experiment using a priori objectively rated global and local landmarks. Although there were definitive differences found between each type, a future experiment will enable a clearer picture with regards to the ways in which people rely on each type of landmark based on their specific locations (internal and peripheral) and within the specific spatial tasks.

Practical implications. The findings produced from this series of studies can be integrated into modeling and simulating human knowledge and behaviour related to wayfinding and spatial cognition. This field can further progress by testing and integrating more fine-grained and valid representations of human behaviour. For example, this knowledge can be integrated into modeling and simulating human way-finding in 
unfamiliar spaces for emergency response crews in order to find ways in which to facilitate facility familiarization. Further, this research could facilitate the way instructions are provided to enable successful way-finding skills. The ability to teach way-finders which world features to attend to in order to facilitate their way-finding in unfamiliar environments could potentially save lives, for example, soldiers once intheatre and emergency responders who are required to way-find with little to no a priori knowledge of the environment (Roberts \& Parush, 2009).

According to (Iaboni \& McGregor, 2009) current tools and techniques used in 3D VEs do not take into consideration the specific needs of the user and their task. Findings from the current thesis suggests that the users' amount of familiarity and spatial task is of utmost importance as it relates to the features in the VE that are attended to and used to way-find and acquire cognitive maps. Therefore, a further practical implication is within the augmentation of virtual environments or aids. This could be done my augmenting the world by adapting its landmarks as a function of familiarity. As evidence was found for a benefit for global landmarks with lower amounts of familiarity an augmented environment or aid, such as a heads up display or you-are-here map, could augment the world by adapting the focus from global to local with increasing familiarity.

The design of 3D VE's should provide adequate cues to ensure that users' wayfinding in complex environments can quickly find their way efficiently and with confidence. First, because each type and location provides different types of information for the user it can be recommended that all combinations of global - local and peripheral - internal are provided. Second, acquiring survey information is critical for efficient way- 
finding in environments that will be visited often. To enable this boundary information provided by global - peripheral landmarks seemed to aid the user in a positive way, these cues should be provided in a clear and visible way. These could also be augmented in a way if they are not visible at all times. Third, although not empirically significant and therefore in need of future research, designers should provide global landmarks that are located internally to provide information to help judge distance and direction. This type of landmark is typically ignored when empirically evaluating the impact of landmarks by their type however the current findings suggest that global landmarks situated internally were helpful to judge direction compared to their local counterpart. Fourth, although not the focus of discussion in this thesis, it was found that those exposed to both global and local landmarks did not perform as well on some tasks when compared to the global-only and local-only experimental groups. Based on this, users may be overwhelmed with too many landmarks. Therefore it is recommended that low-fidelity user trials include an evaluation of the number of landmarks provided and their impact on performance. There may be a "just enough" number to provide that could enhance way-finding while not increasing the users' cognitive workload. Last, when designing navigation aids both types and locations should be referred to. This is recommended as the spatial task varies when using aids, that is maps can be used for orientation, navigating, or acquiring survey knowledge and it was found that the tasks are related to the various landmark features.

\section{Future Directions}

Three suggestions for future directions are provided. Although there are many, these were chosen for the high level of impact they may have on either spatial cognition 
theories, the design of VE and way-finding aids, or the ability to sharpen the details of current findings. First, the addition of eye-tracking as a dependent variable in a similar experiment to provide verification of the attention to landmarks by their type and location across familiarity is discussed. Second, gender is known to have a significant effect on individual differences in spatial cognition and therefore is discussed as a valid future direction. Finally, the usefulness of landmarks by their type and location was found to be dependent on the spatial task and therefore the inclusion of these landmarks within augmented way-finding aids is also discussed.

Future investigations for the specific visual and cognitive attention given to landmarks by their type and location across familiarity and spatial tasks may be better informed by way of eye-tracking. The basic assumption of eye-tracking is that where the subject is looking is directly related to what they are attending to. Eye-tracking can include a number of measures such as saccades, fixations and eye-movements. Eyetracking data, specifically eye movements or scan paths, have been used retrospectively to analyze usability issues and human performance within the human computer interaction domain (Jacob \& Karn, 2003). The temporal differences between global landmark knowledge and local landmark knowledge found in the current studies could be verified by way of measurements of eye fixations and scan paths via eye-tracking. The impact familiarity has on the attention and use of landmarks based on their type and location could be investigated as a function of real-time and not based on three blocks of 5 trials, which was used in Experiment 3. Therefore, an investigation of eye-movement behaviour while users way-find and complete spatial knowledge tasks as a function of 
landmark type and location would provide a more direct measurement for attention to each landmarks feature across familiarity and spatial task.

In this dissertation gender was controlled for, however, it is a known factor in individual differences for spatial tasks such as way-finding. There is evidence that the use of global and local landmarks may depend on gender, and although outside the scope of the current dissertation, a future investigation of gender could provide an interesting follow-up study. Previous studies have found that females attend to the details whereas males attend to the more general structure of an environment (Parush \& Berman, 2004). For example Sandstrom et al. (1998) found that males were better at adapting their search strategies when only geometric information about the room was available. This could have implications to the type of processing strategy one uses to construct the cognitive map. These studies of gender differences in general imply that males and females use different strategies when navigating, with males constructing more accurate models of the environment (e.g., use more survey knowledge) and women tend to use more route descriptions and landmarks (Astur, Ortiz, \& Sutherland, 1998; Parush \& Berman, 2004; Sandstrom, Kaufman, \& Huettel, 1998; Waller, Hunt, \& Knapp, 1998). These findings also suggest that there may be a difference in the way that males and females encode or construct cognitive maps, with males relying on a top-down process whereas females may rely on a bottom-up process. The implication is that difference would be found for use of global versus local landmarks as a function of gender.

It is further recommended that these findings be integrated into navigation aids. Previous work has been done to customize navigation aids and environments. For 
example, breadcrumbs or virtual footprints (Grammenos, Mourouzis, \& Stephanidis, 2006), trailblazing (Iaboni \& McGregor, 2009) and personal landmarks (Darken, Allard, \& Achille, 1999) have been designed and investigated providing some promise to augmented navigation aids. Given that the familiarity, spatial task, and perhaps gender and age are found to rely on landmarks by their type and location in different ways, future work could investigate how these landmarks may provide further augmentation to navigation aids to provide further adaptation for way-finders. A more specific direction to take this research would be to test the impact of landmarks by their type and location within route descriptions. It can be argued that route descriptions displayed within a typical Global Position Service do not provide much if any survey information to the user, but only small sections of routes at one time. This can potentially lead to problems is the user has become disoriented and the system is unavailable at the time. In this situation, the user is left to find novel routes based only on a limited amount of route knowledge.

Of future interest would be the effect that route depictions containing landmarks by their type and location may have on route and survey knowledge acquisition. Researchers who were interested in the effects of a mobile pedestrian navigation system on concurrent route and survey knowledge acquisition found that, as expected, people who were presented with route instructions were not able to acquire accurate survey knowledge of the environment but their route knowledge was quite accurate (Kruger, Aslan \& Zimmer, 2004). When compared to a map, researchers have found that survey knowledge is acquired, but at a significantly slower rate when people learn an 
environment using route descriptions (Parush \& Berman, 2004). Therefore, if global landmarks can provide some amount of survey knowledge within route depictions, perhaps the users of such systems will be able to acquire survey knowledge in less time and be able to rely on their own survey knowledge if the system becomes unreliable. If global landmarks can facilitate survey knowledge and route instructions are known to be worse than maps at facilitating survey knowledge, perhaps the inclusion of global landmarks within route depictions will enhance survey knowledge for way-finders.

In conclusion, the results and synthesis of this dissertation provide the community with significant contributions. First, to my knowledge this is the first study to explicitly investigate the impact of prominent landmark features, type and location, in isolation and in combination. Although there are numerous types of landmark features based on their perceptual elements that can be investigated other than size, (e.g., colour or smell), this study was the first to investigate features in isolation serving as a strong foundation for future investigation using similar paradigms. Second, a testable theoretical framework that attempts to explain the impact of sensory and spatial information provided by landmarks in way-finding and spatial knowledge was proposed in the aim of driving future theoretical research. Third, the investigation of the acquisition of spatial knowledge in reference to landmark features provides additional depth to the body of knowledge associated with spatial knowledge acquisition processes. Finally, this research offered practical recommendations and imperative future directions on how different landmark features could be used in the design and development of navigable virtual environments and aids. 


\section{References}

Aginsky, V., Harris, C., Rensink, R., \& Beusmans, J. (1997). Two strategies for learning a route in a driving simulator. Journal of Environmental Psychology, 17(4), 317331.

Aguirre, G. K., \& Desposito, M. (1997). Environmental knowledge is subserved by separable dorsal/ventral neural areas. Journal of Neuroscience, 17(7), 2512-2518.

Allen, G. L., Kirasic, K. C., Siegel, A. W., \& Herman, J. F. (1979). Developmental issues in cognitive mapping - Selection and utilization of environmental landmarks. Child Development, 50(4), 1062-1070.

Antes, J. R., \& Mann, S. W. (1984). Global-local precedence in picture processing. Psychological Research, 46(3), 247-259.

Appleyard, D. (1970). Styles and methods of structuring a city. Environment and Behavior, 2(1), 100-117.

Astur, R. S., Ortiz, M. L., \& Sutherland, R. J. (1998). A characterization of performance by men and women in a virtual Morris water task: A large and reliable sex difference. Behavioural Brain Research, 93(1-2), 185-190.

Billinghurst, M. \& Weghorst. S. (1995). The use of sketch maps to measure cognitive maps of virtual environments. Proceedings of Virtual Reality Annual International Symposium (VRAIS '95), Research Triangle Park, North Carolina.

Blajenkova, O., Motes, M. A., \& Kozhevnikov, M. (2005). Individual differences in the representations of novel environments. Journal of Environmental Psychology, 25(1), 97-109. 
Bosco, A., Longoni, A. M., \& Vecchi, T. (2004). Gender effects in spatial orientation: Cognitive profiles and mental strategies. Applied Cognitive Psychology, 18, 519532.

Burgess, N. (2008). Spatial cognition and the brain Year in Cognitive Neuroscience 2008, $1124,77-97$.

Burgess, N., \& O'Keefe, J. (2003). Neural representations in human spatial memory. Trends in Cognitive Sciences, 7(12), 517-519.

Burt, T., Holland, R., \& Guilford, T. (1997). Further evidence for visual landmark involvement in the pigeon's familiar area map. Animal Behaviour, 53, 1203-1209.

Cohen, R., \& Schuepfer, T. (1980). The representation of landmarks and routes. Child Development, 51(4), 1065-1071.

Committeri, G., Galati, G., Paradis, A. L., Pizzamiglio, L., Berthoz, A., \& LeBihan, D. (2004). Reference frames for spatial cognition: Different brain areas are involved in viewer-, object-, and landmark-centered judgments about object location. Journal of Cognitive Neuroscience, 16(9), 1517-1535.

Cornell, E. H., \& Hay, D. H. (1984). Childrens acquisition of a route via different media. Environment and Behavior, 16(5), 627-641.

Couclelis, H., Golledge, R. G., Gale, N., \& Tobler, W. (1987). Exploring the anchorpoint hypothesis of spatial cognition. Journal of Environmental Psychology, 7, $99-122$. 
Creem, S. H., \& Proffitt, D. R. (1998). Two memories for geographical slant: Separation and interdependence of action and awareness. Psychonomic Bulletin \& Review, $5(1), 22-36$

Creem, S. H., \& Proffitt, D. R. (2001). Defining the cortical visual systems: "What", "Where", and "How". Acta Psychologica, 107(1-3), 43-68.

Czarnolewski, M. Y., \& Eliot, J. (2000). Preliminary identification of nonlinear correlations for spatial tests and reaction-time tasks. Perceptual Motor Skills, $90(2), 423-434$.

Darken, R. P., Allard, T., \& Achille, L. B. (1999). Spatial orientation and wayfinding in large-scale virtual spaces II - Introduction. Presence-Teleoperators and Virtual Environments, 8(6), III-VI.

Darken, R. P., \& Peterson, B. (2002). Spatial Orientation, Wayfinding, and Representation. In K. M. Stanney (Ed.), Handbook of Virtual Environment Technology (pp. 493-518). New Jersey: Lawrence Erlbaum Assoc.

Darken, R. P., \& Sibert, J. L. (1996). Navigating large virtual spaces. International Journal of Human-Computer Interaction, 8(1), 49-71.

Davidoff, J., Fonteneau, E., \& Fagot, J. (2008). Local and global processing: observations from a remote culture. Cognition, 108(3), 702-709.

Devlin, A. S. (1976). The 'small town' cognitive map: Adjusting to a new environment. In G. T. Moore \& A. H. Golledge (Eds.), Environment knowing: Theories, research and methods (pp. 58-66). Stroudsburg, PA: Downden, Hutchinson \& Ross. 
Edwards, D. M., \& Hardman, L. (1989). Lost In Hyperspace: Cognitive Mapping and Navigation in a Hypertext Environment. In R. McAleese (Ed.), Hypertext: Theory Into Practice. New Jersey: Ablex Publishing Corporation.

Elvins, T. T. (1997). Worldlets: A novel 3D thumbnail affordance for wayfinding in large virtual environments. Ph.D. 9820873, University of California, San Diego, United States -- California. Retrieved November 13, 2009 from http://proquest.umi.com/pqdweb?did=736885411\&Fmt=7\&clientId=13709\&RQT $=309 \& \mathrm{VName}=\mathrm{PQD}$

Elvins, T. T., Nadeau, D. R., Schul, R., \& Kirsh, D. (2001). Worldlets: 3-D thumbnails for wayfinding in large virtual worlds. Presence-Teleoperators and Virtual Environments, 10(6), 565-582.

Evans, G., Brennan, P. L., Skorpanich, M. A., \& Held, D. (1984). Cognitive mapping and elderly adults - Verbal and location memory for urban landmarks. Journal of Gerontology, 39(4), 452-457.

Evans, G. W., Marrero, D. G., \& Butler, P. A. (1981). Environmental learning and cognitive mapping. Environment and Behavior, 13(1), 83-104.

Evans, G. W., Skorpanich, M. A., Garling, T., Bryant, K. J., \& Bresolin, B. (1984). Effects of Stress in Configuration, and Landmarks on Urban Cognition. Journal of Environmental Psychology. 4, 1984, 323-335.

Garling, T., Book, A., \& Lindberg, E. (1984). Cognitive mapping of large-scale environments - The interrelationship of action plans, acquisition, and orientation. Environment and Behavior, 16(1), 3-34. 
Gauthier, M. S., Parush, A., Macuda, T., Tang, D., Craig, G., \& Jennings, S. (2008). The impact of night vision goggles on way-finding performance and the acquisition of spatial knowledge. Human Factors, 50(2), 311-321.

Glenn, B.T. \& Chignell, M.H. (1992). Hypermedia: Design for browsing. In H. R. Hartson \& D. Hix (Eds.), Advances in Human-Computer Interaction, Vol. 3. Norwood, NJ: Ablex Publishing Corporation.

Golledge, R. G. (1992). Place recognition and wayfinding - Making sense of space. Geoforum, 23(2), 199-214.

Golledge, R. G. (1999). Looking back and looking forward. Geographical Analysis, 31(4), 318-323.

Goodale, M., \& Milner, D. (2006). One brain - two visual systems. Psychologist, 19(11), 660-663.

Gould-Beierle, K. L., \& Kamil, A. C. (1996). The use of local and global cues by Clark's nutcrackers, Nucifraga columbiana. Animal Behaviour, 52, 519-528.

Grammenos, D., Mourouzis, A., \& Stephanidis, C. (2006). Virtual prints: Augmenting virtual environments with interactive personal marks. International Journal of Human-Computer Studies, 64(3), 221-239.

Gunther, R., Kazman, R., \& MacGregor, C. (2004). Using 3D sound as a navigational aid in virtual environments. Behaviour \& Information Technology, 23(6), 435-446.

Hegarty, M., Montello, D. R., Richardson, A. E., Ishikawa, T., \& Lovelace, K. (2006). Spatial abilities at different scales: Individual differences in aptitude-test performance and spatial-layout learning. Intelligence, 34(2), 151-176. 
Heth, C. D., Cornell, E. H., \& Alberts, D. M. (1997). Differential use of landmarks by 8and 12-year-old children during route reversal navigation. Journal of Environmental Psychology, 17(3), 199-213.

Hirtle, S. C., \& Jonides, J. (1985). Evidence of hierarchies in cognitive maps. Memory and Cognition, 13(3), 208-217.

Hirtle, S. C., \& Kallman, H. J. (1988). Memory for the locations of pictures: evidence for hierarchical clustering. American Journal of Psychology, 101(2), 159-170.

Hirtle, S. C., \& Sorrows, M. E. (1998). Designing a multi-modal tool for locating buildings on a college campus. Journal of Environmental Psychology, 18(3), 265276.

Holscher, C., Buchner, S. J., Meilinger, T., \& Strube, G. (2009). Adaptivity of wayfinding strategies in a multi-building ensemble: The effects of spatial structure, task requirements, and metric information. Journal of Environmental Psychology, 29(2), 208-219.

Hopkins, W. D., \& Washburn, D. A. (2002). Matching visual stimuli on the basis of global and local features by chimpanzees (Pan troglodytes) and rhesus monkeys (Macaca mulatta). Animal Cognition, 5(1), 27-31.

Hunt, E., \& Waller, D. (1999). Orientation and wayfinding: A review. ONR technical report N00014-96-0380. Arlington, VA: Office of Naval Research.

Iaboni, D., \& McGregor, C. (2009, 14-15 March). Tech-note: Vtrail: Supporting Trailblazing in Virtual Environments. IEEE Symposium on 3D User Interfaces, Lafayette, Louisiana, USA. 
Jacobs, L. F. (2003). The evolution of the cognitive map. Brain Behavior and Evolution, 62(2), 128-139.

Jacobs, L. F., \& Schenk, F. (2003). Unpacking the cognitive map: The parallel map theory of hippocampal function. Psychological Review, 110(2), 285-315.

Jansen-Osmann, P., \& Fuchs, P. (2006). Wayfinding behavior and spatial knowledge of adults and children in a virtual environment - The role of landmarks. Experimental Psychology, 53(3), 171-181.

Jansen-Osmann, P., \& Wiedenbauer, G. (2006). Distance cognition in virtual environmental space: Further investigations to clarify the route-angularity effect. Psychological Research-Psychologische Forschung, 70(1), 43-51.

Jefferies, M. E., \& Yeap, W. K. (2001). The utility of global representations in a cognitive map. Paper presented at the Proceedings of the 2001 Conference on Spatial Information Theory, 233-246.

Kimchi, R. (1992). Primacy of wholistic processing and global/local paradigm: a critical review. Psychological Bulletin, 112(1), 24-38.

Klatzky, R. L. (1998). Allocentric and egocentric spatial representations: Definitions, distinctions, and interconnections. In C. Freksa, C. Habel \& K. F. Wender (Eds.), Spatial cognition - An interdisciplinary approach to representation and processing of spatial knowledge (Lecture Notes in Artificial Intelligence) (pp. 1-17). Berlin: Springer-Verlag. 
Klippel, A., \& Winter, S. (2005). Structural salience of landmarks for route directions. In A. G. Cohn \& D. M. Mark (Eds.), Spatial Information Theory, Proceedings. Vol. 3693, pp. 347-362.

Kuipers, B. (1978). Modeling spatial knowledge. Cognitive Science, 2,129-153.

Kuipers, B. (2000). The Spatial Semantic Hierarchy. Artificial Intelligence, 119(1-2), 191-233.

Lawton, C., \& Kallai, J. (2002). Gender differences in wayfinding strategies and anxiety about wayfinding: A cross-cultural comparison. Sex Roles, 47(9-10), 389-401.

Liben, L. S. (1999). Developing an understanding of external spatial representations. In I. E. Sigel (Ed.), Development of mental representation: Theories and applications (pp. 297-321). Mahwah, NJ: Lawrence Erlbaum Associates.

Liben, L. S. (2000). Map use and the development of spatial cognition: seeing the bigger picture. Developmental Science, 3(3), 270-274.

Lynch, K. (1960). The Image of the City. Cambridge MA: MIT Press.

Maceachren, A. M. (1992a). Application of environmental learning - Theory to spatial knowledge acquisition from maps. Annals of the Association of American Geographers, 82(2), 245-274.

Maceachren, A. M. (1992b). Learning spatial information from maps - Can orientationspecificity be overcome. Professional Geographer, 44(4), 431-443.

Maguire, E. A., Burgess, N., Donnett, J. G., Frackowiak, R. S., Frith, C. D., \& O'Keefe, J. (1998). Knowing where and getting there: a human navigation network. Science, $280(5365), 921-924$. 
Maguire, E. A., Burgess, N., \& O'Keefe, J. (1999). Human spatial navigation: Cognitive maps, sexual dimorphism, and neural substrates. Current Opinions of Neurobiology, 9(2), 171-177.

Maguire, E. A., Frith, C. D., Burgess, N., Donnett, J. G., \& O'Keefe, J. (1998). Knowing where things are parahippocampal involvement in encoding object locations in virtual large-scale space. Journal of Cognitive Neuroscience, 10(1), 61-76.

Maguire, E. A., Spiers, H. J., Good, C. D., Hartley, T., Frackowiak, R. S., \& Burgess, N. (2003). Navigation expertise and the human hippocampus: a structural brain imaging analysis. Hippocampus, 13(2), 250-259.

Makany, T., Karadi, K., Kallai, J., \& Nadel, L. (2002). Interference between verbal concept formation and spatial mental rotation in female subjects. Perceptual and Motor Skills, 95(1), 227-232.

May, A. J., Ross, T., Bayer, S. H., \& Tarkiainen, M. J. (2003). Pedestrian navigation aids: Information requirements and design principles. Personal Ubiquitous Computing, $7,331-338$.

Miller, J. (1981). Global precedence in attention and decision. Journal of Experimental Psychology of Human Perception and Performance, 7(6), 1161-1174.

Miller, J., \& Navon, D. (2002). Global precedence and response activation: evidence from LRPs. Quarterly Journal of Experimental Psychology A, 55(1), 289-310.

Milner, A. D., \& Goodale, M. A. (1993). Visual Pathways to perception and action. Progress in Brain Research, 95, 317-337. 
Montello, D. R. (1998). A new framework for understanding the acquisition of spatial knowledge in large-scale environments. In M. J. Egenhofer \& R. G. Golledge (Eds.), Spatial and temporal reasoning in geographic information systems (pp. 143-154). New York: Oxford University Press.

Montello, D. R., \& Pick, H. L. (1993). Integrating knowledge of vertically aligned largescale spaces. Environment and Behavior, 25(4), 457-484.

Navon, D. (1977). Forest before trees - Precedence of global features in visualperception. Cognitive Psychology, 9(3), 353-383.

Navon, D. (1983). How many trees does it take to make a forest? Perception, 12(3), 239254.

Navon, D. (1991). Testing a queue hypothesis for the processing of global and local information. Journal of Experimental Psychology: General, 120(2), 173-189.

Navon, D. (2003). What does a compound letter tell the psychologist's mind? Acta Psychologica, 114(3), 273-309.

Navon, D., \& Norman, J. (1983). Does global precedence really depend on visual angle? Journal of Experimental Psychology: Human Perception and Performance, 9(6), 955-965.

Neggers, S. F. W., Van der Lubbe, R. H. J., Ramsey, N. F., \& Postma, A. (2006). Interactions between ego- and allocentric neuronal representations of space. NeuroImage, 31(1), 320-331.

Nielsen, J. (1995). Multimedia and Hypertext: The Internet and Beyond. AP Professional, Boston. 
Newman, E. L., Caplan, J. B., Kirschen, M. P., Korolev, I. O., Sekuler, R., \& Kahana, M. J. (2007). Learning your way around town: How virtual taxicab drivers learn to use both layout and landmark information. Cognition, 104(2), 231-253.

O'Keefe, J., Burgess, N., Donnett, J. G., Jeffery, K. J., \& Maguire, E. A. (1998). Place cells, navigational accuracy, and the human hippocampus. Philosophical Transactions of the Royal Society of London Series B-Biological Sciences, 353(1373), 1333-1340.

Paquet, L., \& Merikle, P. M. (1988). Global precedence in attended and nonattended objects. Journal of Experimental Psychology: Human Perception and Performance, 14(1), 89-100.

Parush, A., \& Berman, D. (2004). Navigation and orientation in 3D user interfaces: the impact of navigation aids and landmarks. International Journal of HumanComputer Studies, 61(3), 375-395.

Peters, M., Laeng, B., Latham, K., Jackson, M., Zaiyouna, R., \& Richardson, C. (1995). A redrawn Vandenberg and Kuse Mental Rotations Test - Different versions and factors that affect performance. Brain and Cognition, 28(1), 39-58.

Pomerantz, J. R. (1983). Global and local precedence: selective attention in form and motion perception. Journal of Experimental Psychology: General, 112(4), 516540.

Presson, C. C., \& Montello, D. R. (1988). Points of reference in spatial cognition Stalking the elusive landmark. British Journal of Developmental Psychology, 6, $378-381$. 
Roberts, S., \& Parush, A. (2009). Role of landmark size and location in way-finding and spatial cognition in virtual environments. Paper presented at the Proceedings of the Human Factors and Ergonomics Society 53rd Annual Meeting, San Antonio, Texas, 2019-2023.

Ruddle, R. A., Payne, S. J., \& Jones, D. M. (1997). Navigating buildings in "desk-top" virtual environments: Experimental investigations using extended navigational experience. Journal of Experimental Psychology-Applied, 3(2), 143-159.

Ruddle, R. A., \& Peruch, P. (2004). Effects of proprioceptive feedback and environmental characteristics on spatial learning in virtual environments. International Journal of Human-Computer Studies, 60(3), 299-326.

Rumpradit, C., \& Donnell, M. L. (1999). Navigational Cues on User Interface Design to Produce Better Information Seeking on the World Wide Web. Paper presented at the Hawaii International Conference on System Sciences.

Sandstrom, N. J., Kaufman, J., \& Huettel, S. A. (1998). Males and females use different distal cues in a virtual environment navigation task. Cognitive Brain Research, $6(4), 351-360$.

Satalich, G. (1995). Navigation and wayfinding in virtual reality: finding proper tool and cues to enhance navigation awareness. Master's thesis, University of Seattle, Seattle, WA. Retrieved from http://www.hitl.washington.edu/publications/satalich. 
Save, E., \& Poucet, B. (2000). Involvement of the hippocampus and associative parietal cortex in the use of proximal and distal landmarks for navigation. Behavioural Brain Research, 109(2), 195-206.

Siegel, A., \& White, S. (1975). The development of spatial representations of large-scale environments. In H. Reese (Ed.), Advances in Child Development and Behavior, 10, 9-55. New York: Academic Press.

Sorrows, M. E. (2005). Recall of landmarks in information space. Ph.D. 3193008, University of Pittsburgh, Pennsylvania. Retrieved from http://proquest.umi.com/pqdweb?did=997899051\&Fmt=7\&clientId=13709\&RQT $=309 \& \mathrm{VName}=\mathrm{PQD}$

Spencer, J. P., Simmering, V. R., \& Schutte, A. R. (2006). Toward a formal theory of flexible spatial behavior: Geometric category biases generalize across pointing and verbal response types. Journal of Experimental Psychology-Human Perception and Performance, 32(2), 473-490.

Stankiewicz, B. J., \& Kalia, A. A. (2007). Acquisition of structural versus object landmark knowledge. Journal of Experimental Psychology-Human Perception and Performance, 33(2), 378-390.

Steck, S. D., \& Mallot, H. A. (1999). Different strategies of global and local landmark usage in virtual environment navigation. Journal of Cognitive Neuroscience, 7677. 
Steck, S. D., \& Mallot, H. A. (2000). The role of global and local landmarks in virtual environment navigation. Presence-Teleoperators and Virtual Environments, 9(1), 69-83.

Steck, S. D., Mochnatzki, H. F., \& Mallot, H. A. (2003). The role of geographical slant in virtual environment navigation. In C. Freksa, W. Brauer, C. Habel \& K. F. Wender (Eds.), Spatial Cognition, 2685, 62-76.

Strasser, R., Bingman, V. P., Ioale, P., Casini, G., \& Bagnoli, P. (1998). The homing pigeon hippocampus and the development of landmark navigation. Developmental Psychobiology, 33(4), 305-315.

Thorndyke, P. W., \& Hayes-Roth, B. (1982). Differences in spatial knowledge acquired from maps and navigation. Cognitive Psychology, 14(4), 560-589.

Tlauka, M., \& Wilson, P. N. (1994). The effect of landmarks on route-learning in a computer simulated environment. Journal of Environmental Psychology, 14(4), 305-313.

Tolman, E. C. (1948). Cognitive maps in rats and men. Psychological Review, 55(4), 189-208.

Tversky, B. (1991). Spatial mental models. Psychology of Learning and MotivationAdvances in Research and Theory, 27, 109-145.

Tversky, B. (2003). Structures of mental spaces - How people think about space. Environment and Behavior, 35(1), 66-80. 
Tversky, B., Franklin, N., Taylor, H. A., \& Bryant, D. J. (1994). Spatial mental models from descriptions. Journal of the American Society for Information Science, 45(9), 656-668.

Vandenberg, S. G., \& Kuse, A. R. (1978). Mental rotation: A group test of threedimensional spatial visualization. Perceptual and Motor Skills, 47, 599-604.

Vinson, N. G. (1999). Design guidelines for landmarks to support navigation in virtual environments. In Proceedings of the SIGCHI conference on Human factors in computing systems: the $\mathrm{CHI}$ is the limit, 278-285, May 15-20, Pittsburgh, Pennsylvania, United States.

Waller, D., Hunt, E., \& Knapp, D. (1998). The transfer of spatial knowledge in virtual environment training. Presence-Teleoperators and Virtual Environments, 7(2), 129-143.

Werner, S., \& Long, P. (2003). Cognition meets le corbusier - Cognitive principles of architectural design. In C. Freksa, W. Brauer, C. Habel \& K. F. Wender (Eds.), Spatial Cognition, 2685, 112-126.

Wiener, J. M., Schnee, A., \& Mallot, H. A. (2004). Use and interaction of navigation strategies in regionalized environments. Journal of Environmental Psychology, $24,475-493$.

Winter, S. (2003). Route adaptive selection of salient features. In W. Kuhn, M. Worboys \& S. Timpf (Eds.), Spatial Information Theory, Proceedings - Foundations of Geographic Information Science. Vol. 2825, pp. 349-361. 
Yeap, W. K., \& Jefferies, M. E. (1997). Computing a representation of the local environment. Computer Science University of Otago. Otago. 


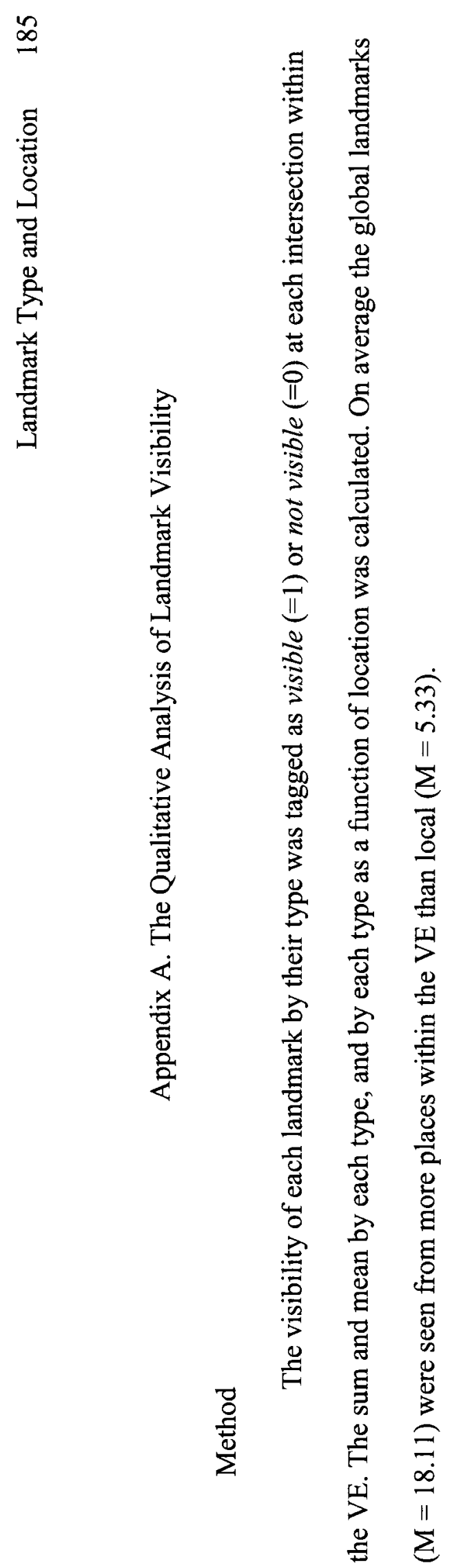




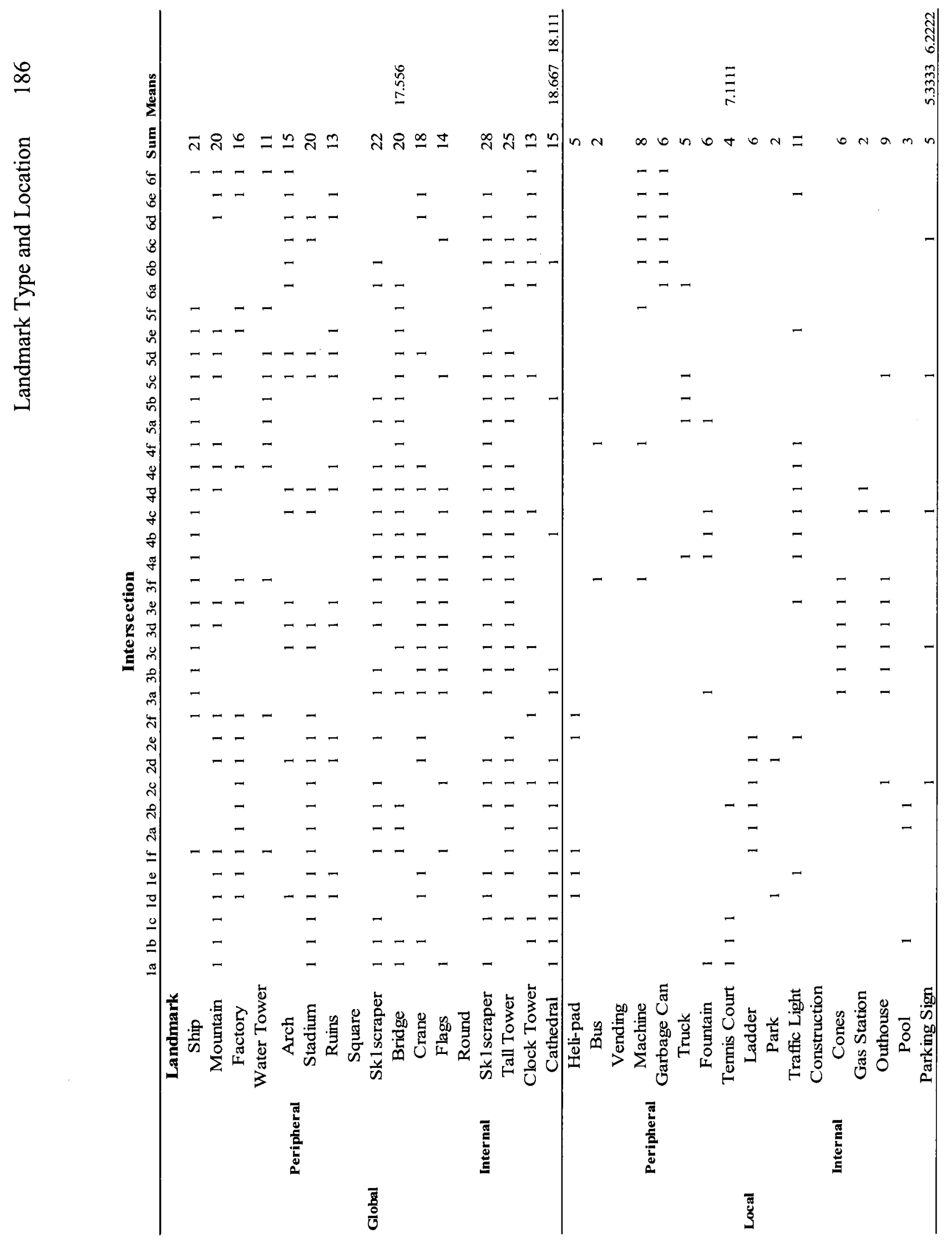


Appendix B. Landmarks by Name, Type, and Location

\begin{tabular}{|c|c|c|c|}
\hline Name & Type & Location & Picture \\
\hline Ship & Global & Peripheral & \\
\hline Mountain & Global & Peripheral & \\
\hline Factory & Global & Peripheral & \\
\hline Water Tower & Global & Peripheral & \\
\hline Arch & Global & Peripheral & \\
\hline Stadium & Global & Peripheral & \\
\hline Ruins & Global & Peripheral \\
\hline Crane & Global & Internal \\
\hline Gridge & Global & Peripheral & \\
\hline Global & Insternal & \\
\hline
\end{tabular}




\begin{tabular}{|c|c|c|c|}
\hline Round Skyscraper & Global & Internal & \\
\hline Tall Tower & Global & Internal & \\
\hline Clock Tower & Global & Internal & \\
\hline Cathedral & Global & Internal & \\
\hline Heli-pad & Local & Peripheral & \\
\hline Bus & Local & Peripheral \\
\hline Truck & Local & Peripheral & \\
\hline Gending Machine & Local & Peripheral \\
\hline
\end{tabular}




\begin{tabular}{|c|c|c|c|}
\hline Ladder & Local & Internal & Internal \\
\hline Park & Local & Internal & \\
\hline Traffic Light & Local & Internal & \\
\hline Construction Cones & Local & Internal & \\
\hline Gas Station & Local & Internal & \\
\hline Outhouse & Local & Internal \\
\hline Pool & Local & & \\
\hline
\end{tabular}




\section{MENTAL ROTATIONS TEST (MRT-A)}

This test is composed of the figures provided by Shepard and Metzler (1978), and is, essentially, an Autocadredrawn version of the Vandenberg \& Kuse MRT test.

CMichael Peters, PhD, July 1995

Please look at these five figures
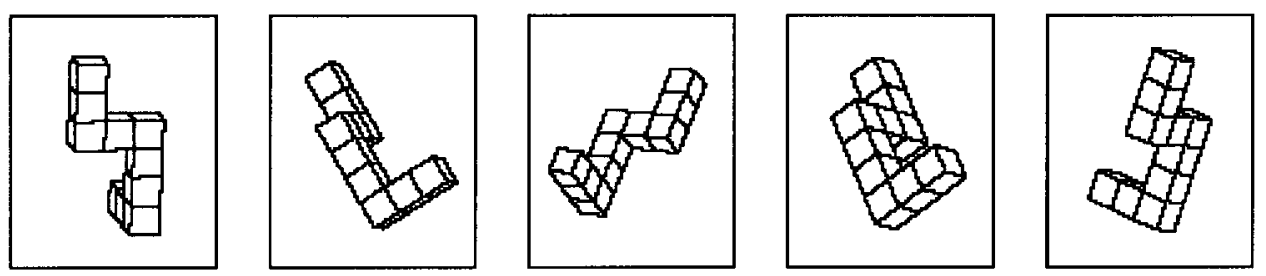

Note that these are all pictures of the same object which is shown from different angles. Try to imagine moving the object (or yourself with respect to the object), as you look from one drawing to the next.
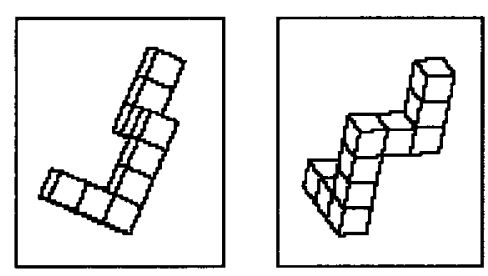

Here are two drawings of a new figure that is different from the one shown in the first 5 dra wings. Satisfy yourself that these two drawings show an object that is different and cannot be "rotated" to be identical wi ith the object shown in the furst five drawings.

Now look at

this object:

1 .

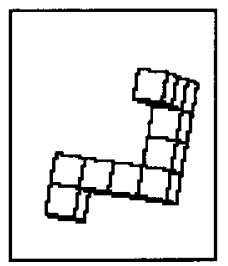

Two of these four drawings show the same object. Can you find those two? Put a big $\mathrm{X}$ across them.
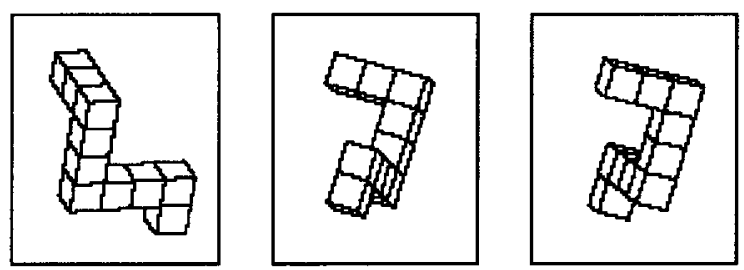

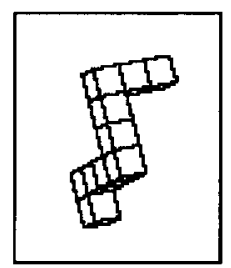

If you marked the first and third drawings, you made the correct chnice. 
Appendix D. Pre and Post-Cybersickness Questionnaire

Simulator Sickness Questionnaire: Symptom Checklist

Please fill in this questionnaire. Circle below if any of the symptoms apply to you now. You will be asked to fill this again after the experiment.

\begin{tabular}{|c|c|c|c|c|c|c|}
\hline 1. General discomfort & None & Slight & & \multicolumn{2}{|c|}{ Moderate } & Severe \\
\hline 2. Fatigue & None & Slight & \multicolumn{3}{|c|}{ Moderate } & Severe \\
\hline 3. Boredom & None & Slight & \multicolumn{3}{|c|}{ Moderate } & Severe \\
\hline 4. Drowsiness & None & Slight & \multicolumn{3}{|c|}{ Moderate } & Severe \\
\hline 5. Headache & None & Slight & \multicolumn{3}{|c|}{ Moderate } & Severe \\
\hline 6. Eyestrain & None & Slight & \multicolumn{3}{|c|}{ Moderate } & Severe \\
\hline 7. Difficulty focusing & None & Slight & \multicolumn{3}{|c|}{ Moderate } & Severe \\
\hline $\begin{array}{l}\text { 8. Salivation increase } \\
\text { Salivation decrease }\end{array}$ & $\begin{array}{l}\text { None } \\
\text { None }\end{array}$ & $\begin{array}{l}\text { Slight } \\
\text { Slight }\end{array}$ & \multicolumn{3}{|c|}{$\begin{array}{l}\text { Moderate } \\
\text { Moderate }\end{array}$} & $\begin{array}{l}\text { Severe } \\
\text { Severe }\end{array}$ \\
\hline 9. Sweating & None & Slight & \multicolumn{3}{|c|}{ Moderate } & Severe \\
\hline 10. Nausea & None & Slight & \multicolumn{3}{|c|}{ Moderate } & Severe \\
\hline 11. Difficulty & None & Slight & \multicolumn{3}{|c|}{ Moderate } & Severe \\
\hline 12. Mental depression & & No & Yes ( & Slight & Moderate & Severe ) \\
\hline 13. "Fullness of the head" & & No & Yes ( & Slight & Moderate & Severe ) \\
\hline 14. Blurred vision & No & Yes ( $S$ & light & Moderat & Severe $)$ & ) \\
\hline 15. Dizziness eyes open & & No & Yes ( & Slight & Moderate & Severe ) \\
\hline Dizziness eyes close & & No & Yes ( & Slight & Moderate & Severe ) \\
\hline 16. Vertigo & & No & Yes ( & Slight & Moderate & Severe ) \\
\hline 17. Visual flashbacks & & No & Yes ( & Slight & Moderate & Severe ) \\
\hline 18. Faintness & & No & Yes ( & Slight & Moderate & Severe ) \\
\hline 19. Aware of breathing & & No & Yes ( & Slight & Moderate & Severe ) \\
\hline 20. Stomach awareness & & No & Yes ( & Slight & Moderate & Severe ) \\
\hline 21. Loss of appetite & & No & Yes ( & Slight & Moderate & Severe ) \\
\hline 22. Increased appetite & & No & Yes ( & Slight & Moderate & Severe ) \\
\hline 23. Desire to move bowels & No & Yes ( $S$ & light & Moderat & Severe ) & ) \\
\hline 24. Confusion & & No & Yes ( & Slight & Moderate & Severe \\
\hline
\end{tabular}


25. Burping

26. Vomiting

27. Other

$\begin{array}{llll}\text { No } & \text { Yes ( Slight } & \text { Moderate } & \text { Severe) } \\ \text { No } & \text { Yes ( Slight } & \text { Moderate } & \text { Severe) } \\ \text { No } & \text { Yes ( Slight } & \text { Moderate } & \text { Severe) }\end{array}$


Appendix E. Secondary Tasks: Procedure, Questions, and Results

Secondary Tasks: Analyses related to Judgement of Relative Direction (JRD) and Relative Distance Estimation (RDE) measures are not discussed in the body of this dissertation. The procedure, measurements, and results are described in this appendix.

1. Procedure for JRD and RDE:

a. Judgment of Relative Direction (JRD): Participants estimated the relative direction towards non-visible landmarks from a vantage point of a target location. This task has been used successfully to measure participants' orientation performance (Blajenkova, et al., 2005; Parush \& Berman, 2004). Participants were asked to estimate the relative direction of a specific landmark (in this example the clock tower) from their current vantage point using one of the 12 arrows, spaced by $30^{\circ}$ increments. In this example the participants are asked to point in the direction of the clock tower using the arrows provided.

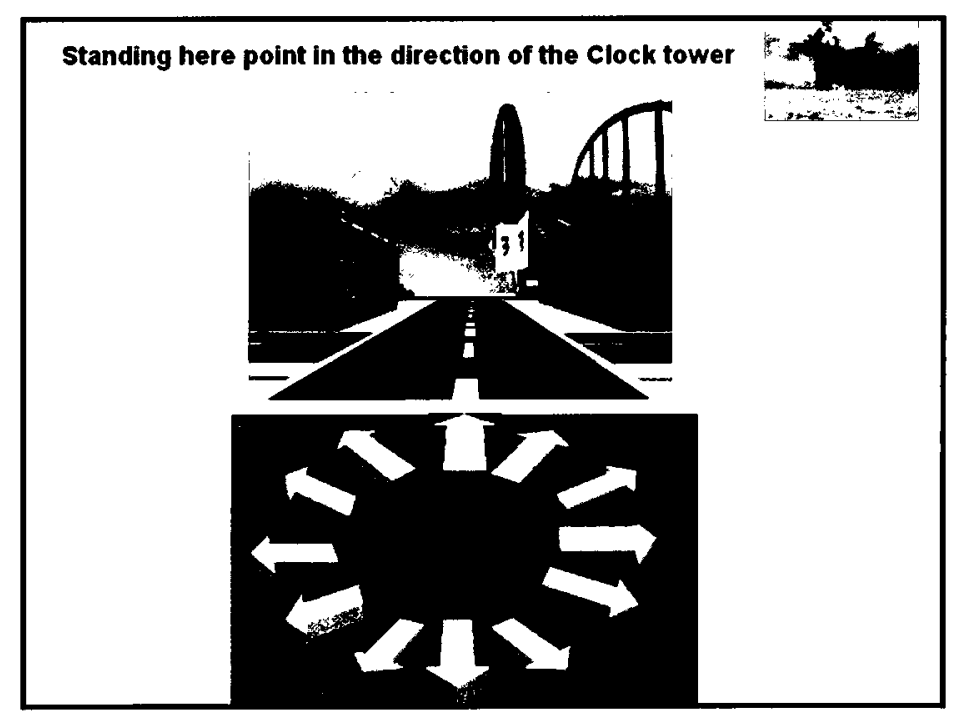


An example of a Judgment of Relative Direction question.

b. Relative Distance Estimation (RDE): Participants estimated the shortest straight-line distance between two landmarks. This task probed participants' survey knowledge as contextual knowledge was not provided within the questions. For example, as shown in Error! Reference source ot found. figure below, participants were asked to judge, from memory, which landmark, out of three choices, was closer to the test landmark displayed in the top right-hand corner. The Both group received questions that referred to both global and local landmarks, Global-only group received questions that referred to only global landmarks and Local-only group received questions that only referred to local landmarks.

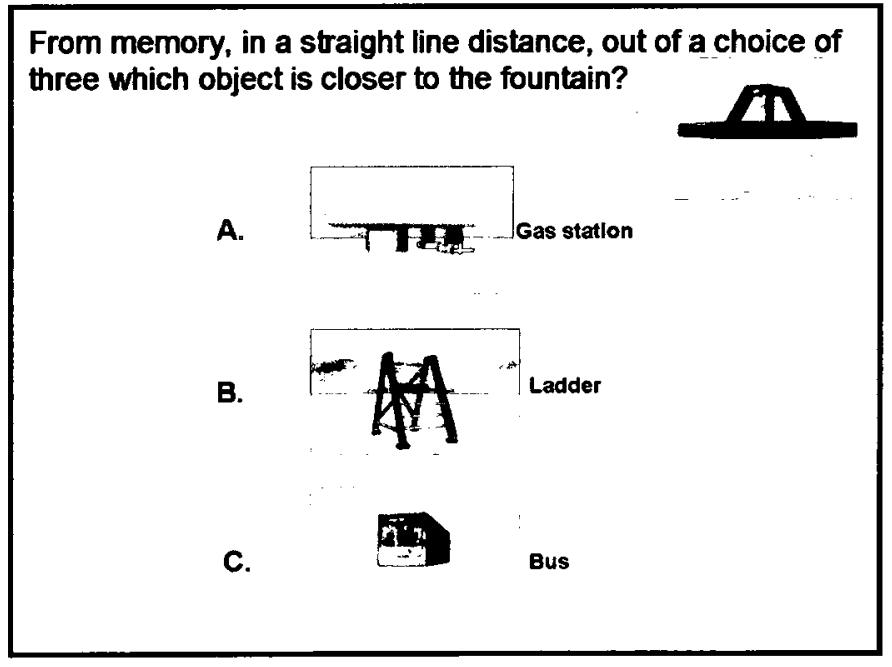

An example of a Relative Direction question.

2. Secondary Task Measurements: 
a. Degree of deviation: The mean absolute deviation, in degrees, between the correct and chosen direction to the test landmark within the JRD questions.

b. Distance Estimation: The mean number of correct RDE answers.

3. Questions:

a. JRD questions: A list of all JRD questions received by each group in all three experiments as labeled.

Experiment 1: Both group and Experiment 3: All groups

\begin{tabular}{ll}
\hline $\begin{array}{l}\text { Standing here point in } \\
\text { the direction of the: }\end{array}$ & Answer in \\
\hline degrees
\end{tabular}


Experiment 1: Global-only group

\begin{tabular}{|c|c|}
\hline $\begin{array}{l}\text { Standing here point in the } \\
\text { direction of the following: }\end{array}$ & $\begin{array}{l}\text { Answer in } \\
\text { degrees }\end{array}$ \\
\hline Construction crane & 60 \\
\hline Stadium & 300 \\
\hline Clock tower & 240 \\
\hline Tall tower & 60 \\
\hline Round skyscraper & 300 \\
\hline Arch & 90 \\
\hline Mountain & 270 \\
\hline Water tower & 210 \\
\hline Ship & 300 \\
\hline Cathedral & 120 \\
\hline Roman temple & 330 \\
\hline Bridge & 240 \\
\hline
\end{tabular}


Experiment 1: Local-only group

\begin{tabular}{ll}
\hline $\begin{array}{l}\text { Standing here point in the } \\
\text { direction of the following: }\end{array}$ & $\begin{array}{l}\text { Answer in } \\
\text { degrees }\end{array}$ \\
\hline Tennis court & 120 \\
Gas station & 60 \\
Road sign & 210 \\
Pool & 240 \\
Traffic light & 150 \\
Park & 90 \\
Garbage bin & 90 \\
Truck & 330 \\
Bus & 270 \\
Fountain & 30 \\
Ladder & 90 \\
Heli-pad & 300 \\
\hline
\end{tabular}


Experiment 2: Global-Peripheral group

\begin{tabular}{ll}
\hline $\begin{array}{l}\text { Standing here point in the } \\
\text { direction of the following: }\end{array}$ & Answer in \\
\hline degrees \\
Arch & 300 \\
Mountain & 90 \\
Water tower & 270 \\
Ship & 210 \\
Cathedral & 300 \\
Bridge & 120 \\
Factory & 240 \\
Square skyscraper & 150 \\
Arch & 270 \\
Bridge & 270 \\
Stadium & 300 \\
\hline
\end{tabular}


Experiment 2: Global-Internal group

\begin{tabular}{ll}
\hline Standing here point in the & Answer in \\
direction of the following: & degrees \\
\hline Cn-tower & 300 \\
Roman ruins & 90 \\
Clock tower & 270 \\
Crane & 90 \\
Roman ruins & 270 \\
Crane & 270 \\
Clock tower & 30 \\
Cn-tower & 90 \\
Crane & 300 \\
Flags & 30 \\
Cn-tower & 60 \\
Flags & 330 \\
\hline
\end{tabular}


Experiment 2: Local-Peripheral group

\begin{tabular}{ll}
\hline $\begin{array}{l}\text { Standing here point in the } \\
\text { direction of the following: }\end{array}$ & Answer \\
\hline in degrees \\
Vending court & 120 \\
Garbage can & 60 \\
Heli-pad & 270 \\
Fountain & 270 \\
Truck & 300 \\
Bus & 210 \\
Garbage can & 180 \\
Heli-pad & 210 \\
Fountain & 90 \\
Tennis Court & 60 \\
Bus & 150 \\
\hline
\end{tabular}


Experiment 2: Local-Internal group

\begin{tabular}{ll}
\hline Standing here point in the & Answer in \\
direction of the following: & degrees \\
\hline Gas station & 60 \\
Park & 90 \\
Ladder & 330 \\
Gas station & 30 \\
Cones & 330 \\
Pool & 120 \\
Outhouse & 60 \\
Pool & 270 \\
Parking sign & 90 \\
Outhouse & 180 \\
Pool & 270 \\
Park & 180 \\
\hline
\end{tabular}




\section{RDE questions:}

Experiment 1: Both group and Experiment 3: All groups

\section{Question 1}

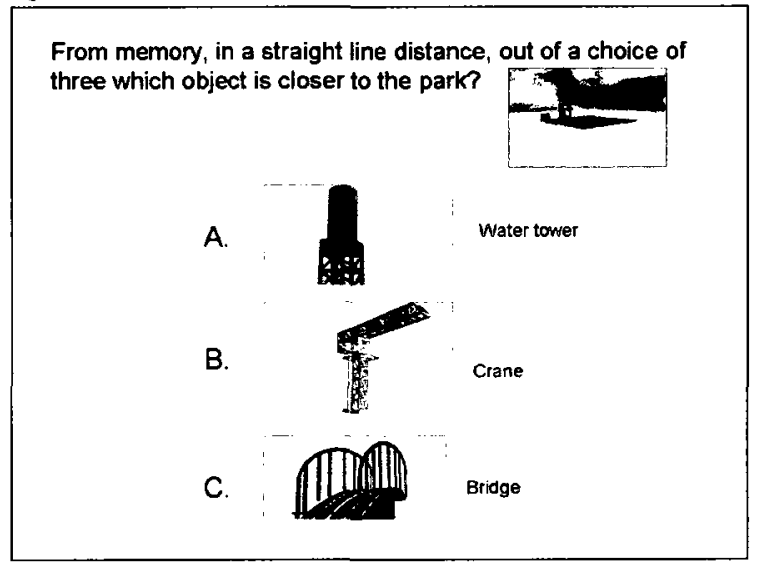

Question 3

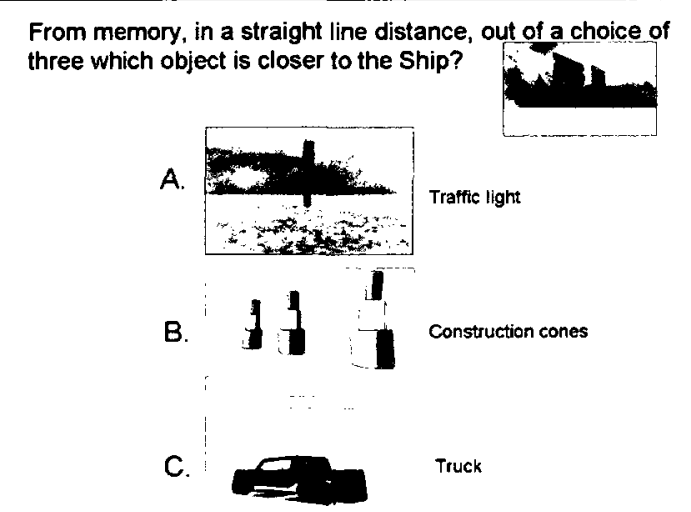

Question 5

From memory, in a straight line distance, out of a choice of three which object is closer to the Roman temple?

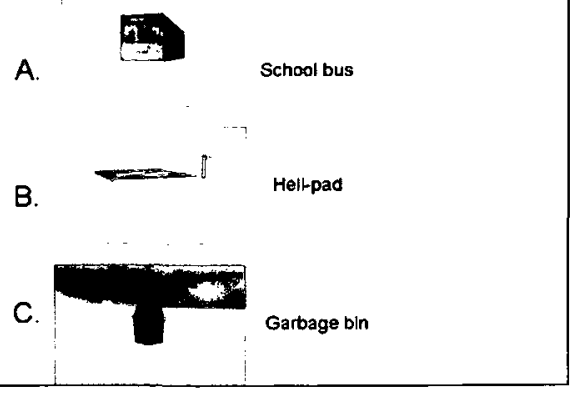

Question 2

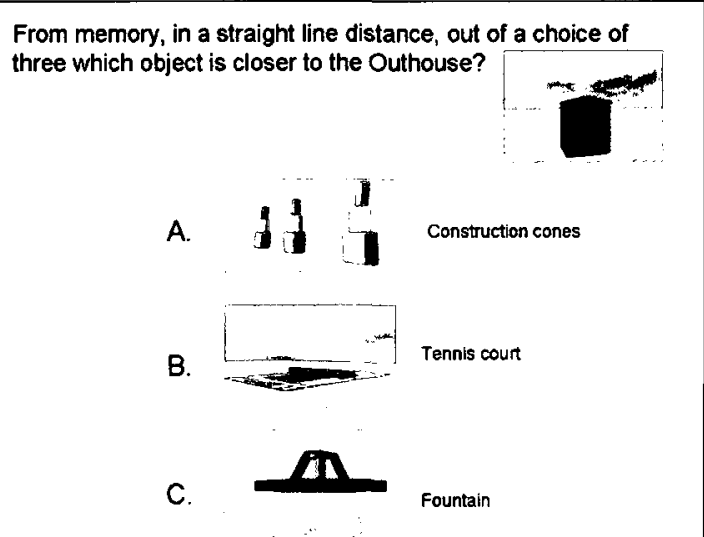

\section{Question 4}

From memory, in a straight line distance, out of a choice of three which object is closer to the crane?

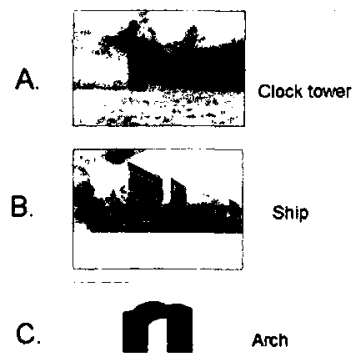

\section{Question 6}

From memory, in a straight line distance, out of a choice of three which object is closer to the tennis court?

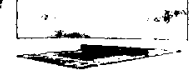

A. Bustoris stadium

B.

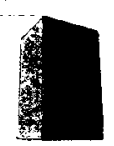

Square skyscraper

c.

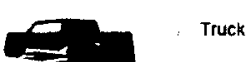




\section{Question 7}

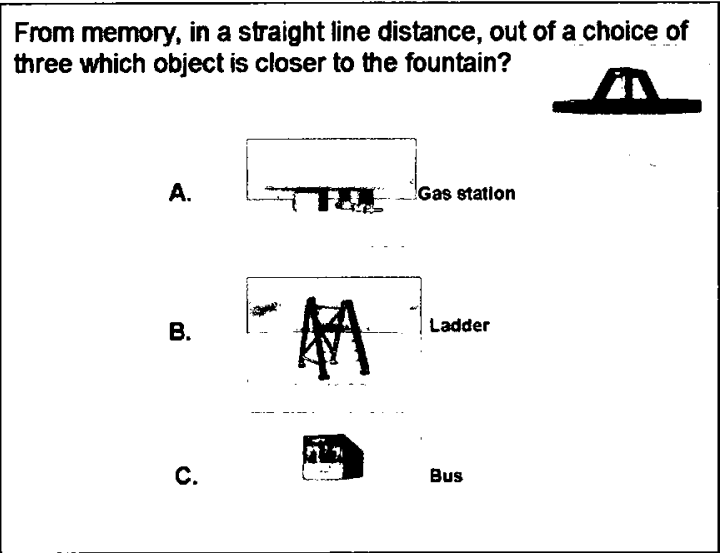

\section{Question 9}

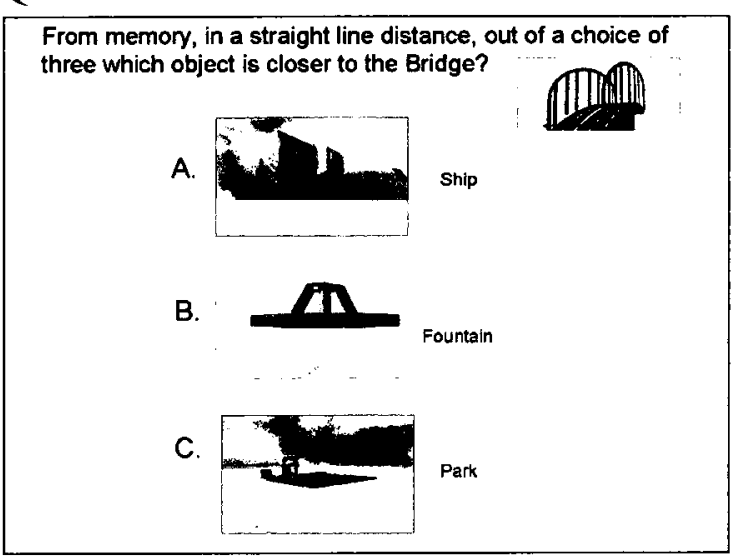

\section{Question 11}

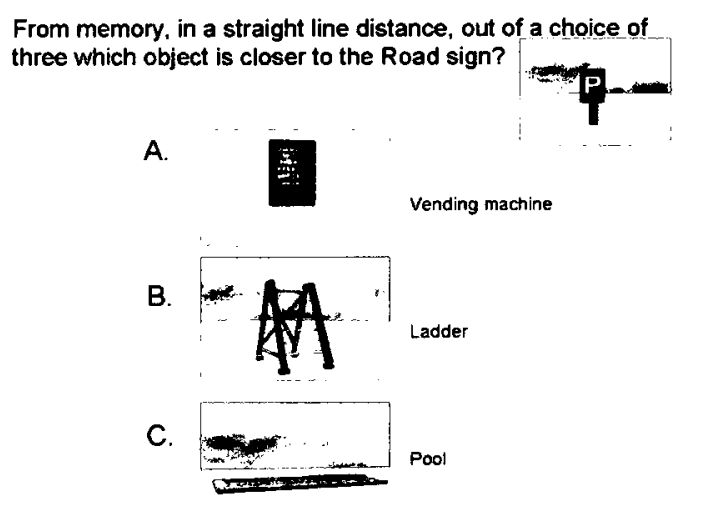

Question 8

From memory, in a straight line distance, out of a choice of three which object is closer to the Mountain?

A.
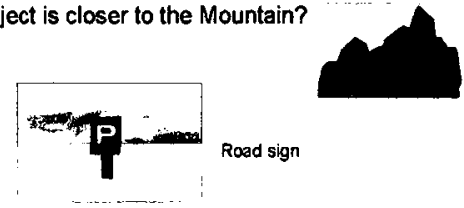

B.

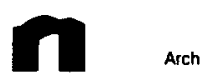

C.

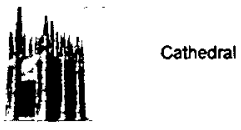

\section{Question 10}

From memory, in a straight line distance, out of a choice of three which object is closer to the School bus?

A.

Round skyscraper

B.

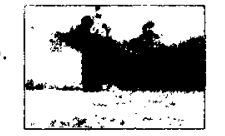

Clock tower

C.

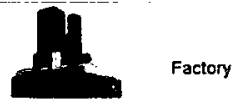

\section{Question 12}

From memory, in a straight line distance, out of a choice of three which object is closer to the Arch?

A.

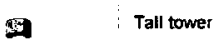

B.

0

Water tower

C.

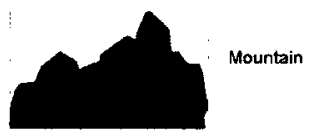




\section{Experiment 1: Global-only Group}

\section{Question 1}

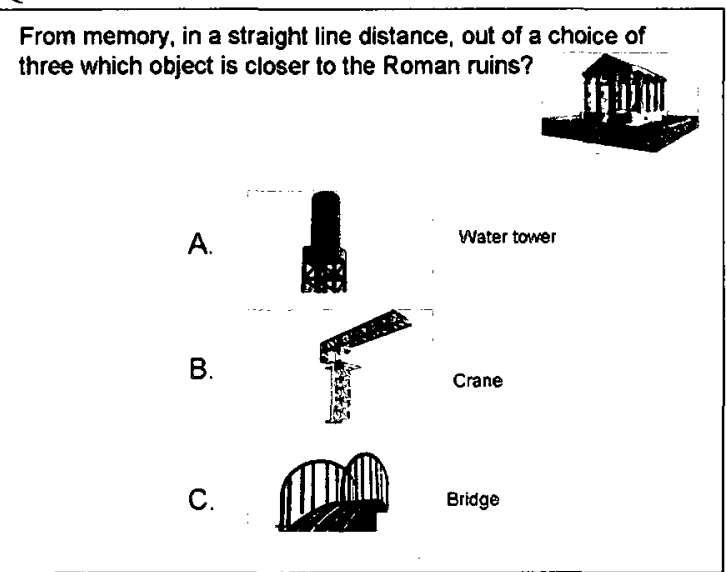

\section{Question 3} From memory, in a straight line distance, out of a choice of
three which object is closer to the Arch?

A.

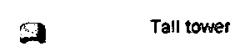

B.

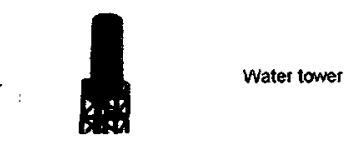

C.

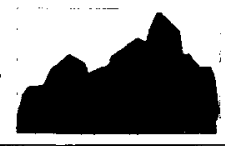

Mountain

\section{Question 2}

From memory, in a straight line distance, out of a choice of three which object is closer to the Crane?

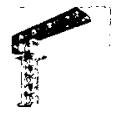

A.

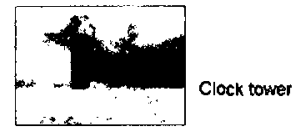

B.

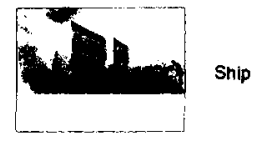

C. Arch

\section{Question 4}

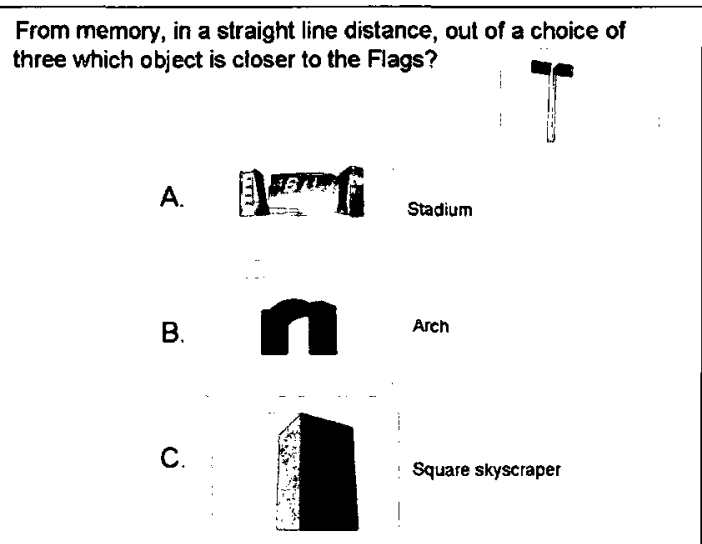

\section{Question 6}

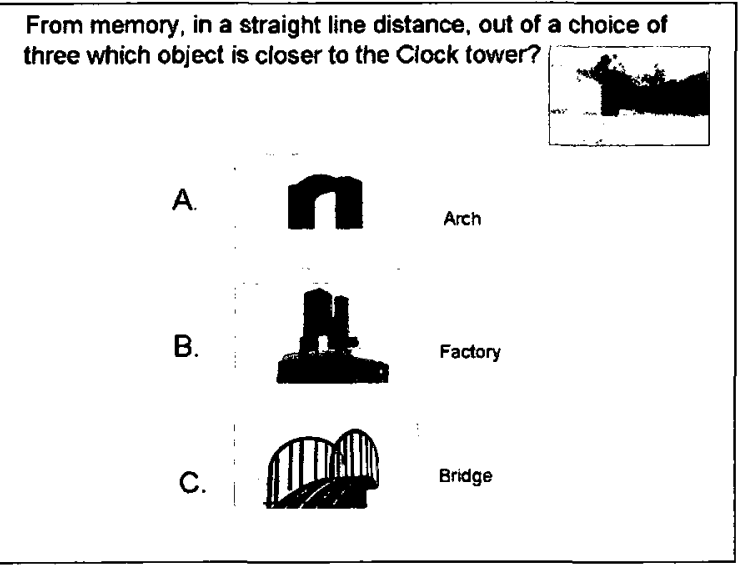

From memory, in a straight line distance, out of a choice of three which object is closer to the Mountain?

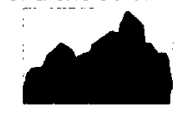
A.

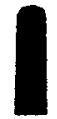
B.

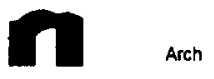
C.

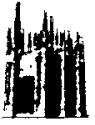

Round skyscraper 


\section{Question 7}

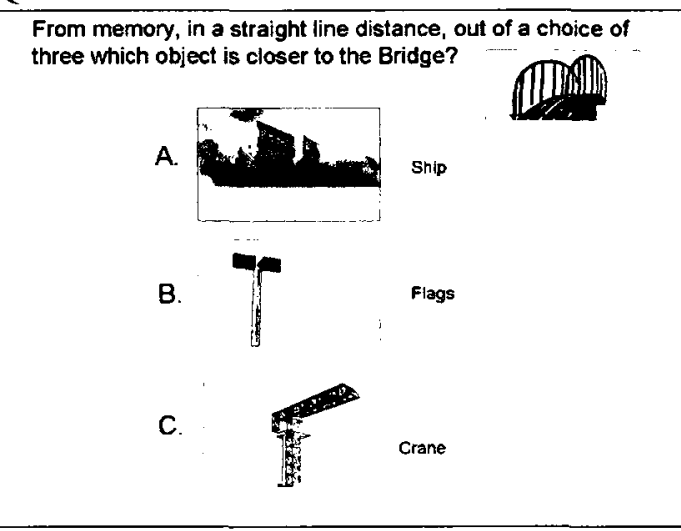

\section{Question 9}

From memory, in a straight line distance, out of a choice of three which object is closer to the Arch?

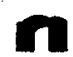

A.

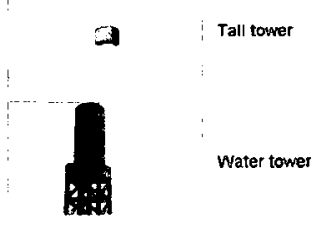

B.

C.

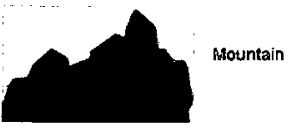

\section{Question 11}

From memory, in a straight line distance, out of a choice of three which object is closer to the Cathedral?

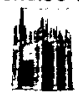

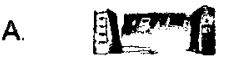
stadium
B.

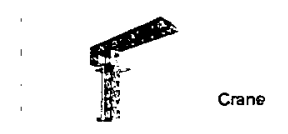
C.

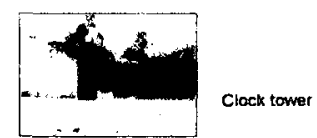

\section{Question 8}

From memory, in a straight line distance, out of a choice of three which object is closer to the Tall tower?

a

A.

Round skyscraper

B.

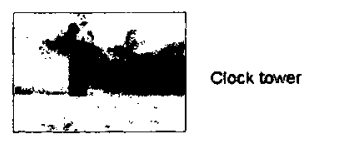

c.

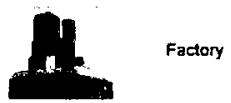

\section{Question 10}

From memory, in a straight line distance, out of a choice of three which object is closer to the Water tower?

A.

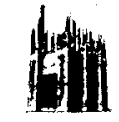

Cathedral

B.

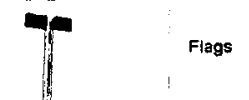

c.

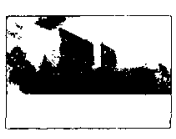

Ship

\section{Question 12}

From memory, in a straight line distance, out of a choice of three which object is closer to the Ship?

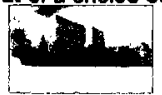

A.

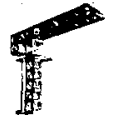

Crane

B.

a) Tall tower

C.

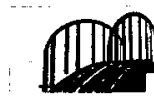

Bridge 


\section{Experiment 1: Local-only Group:}

Question 1

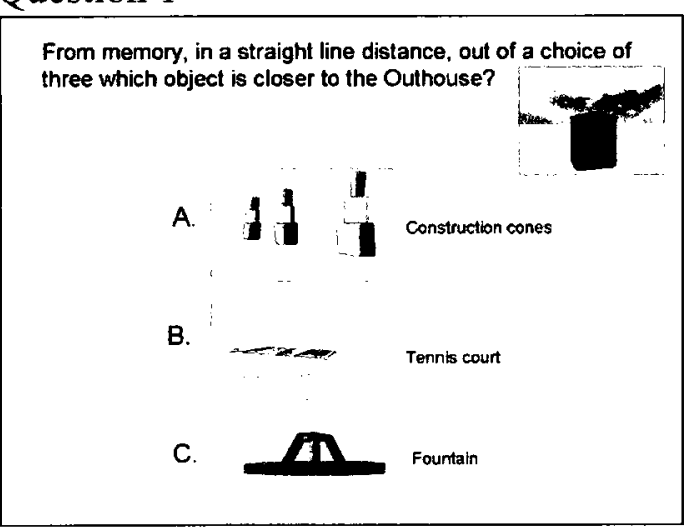

\section{Question 3}

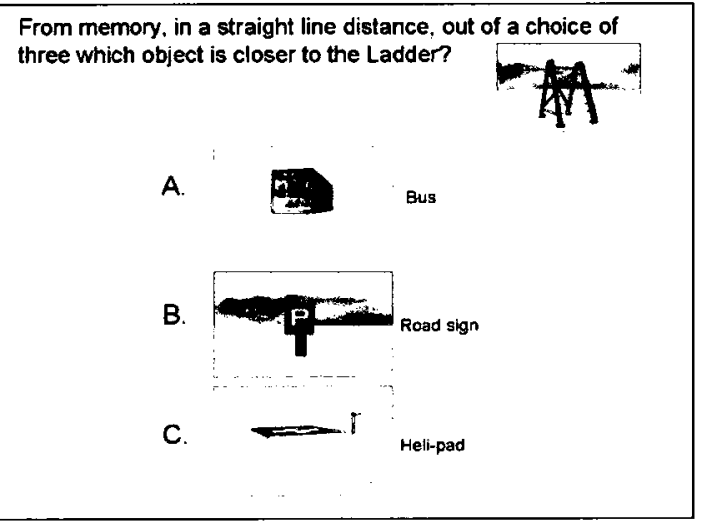

\section{Question 5}

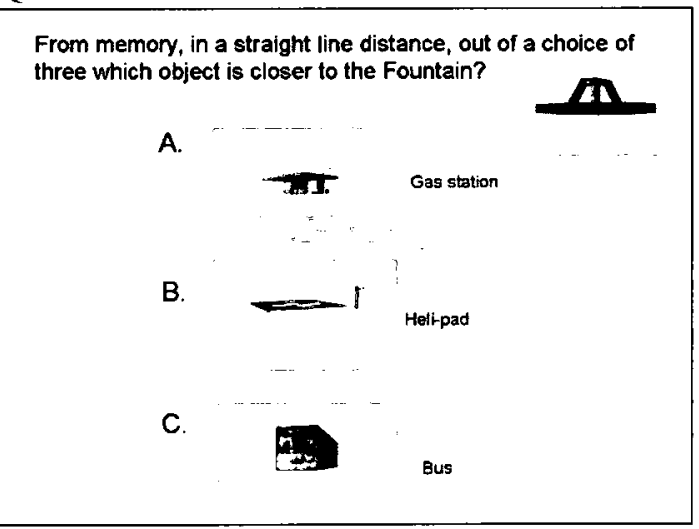

\section{Question 2}

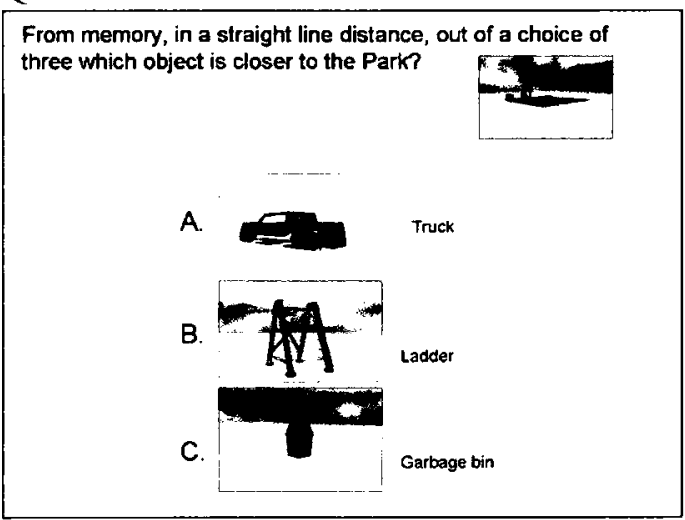

\section{Question 4}

From memory, in a straight line distance, out of a choice of three which object is closer to the Pool?
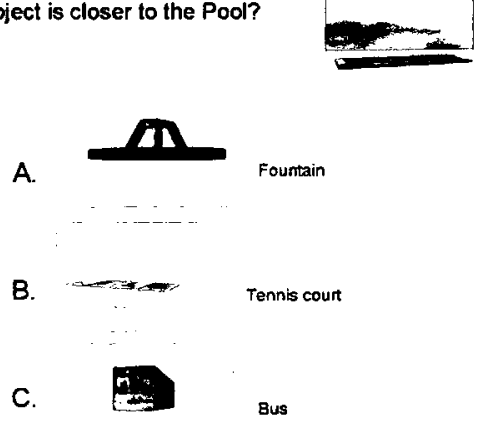

Question 6

From memory, in a straight line distance, out of a choice of three which object is closer to the Fountain?

a

A.

2.1. Gas station

B.

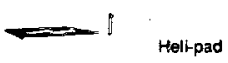

C.

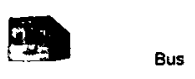


Question 7

From memory, in a stralght line distance, out of a choice of three which object is closer to the Garbage bin?

A.

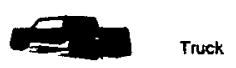

B.

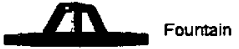

c.

Vending machine

\section{Question 8}

From memory, in a straight line distance, out of a choice of three which object is closer to the Truck?

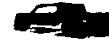

A

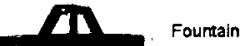

B. $11 \frac{1}{1}$ Construction cones

C.

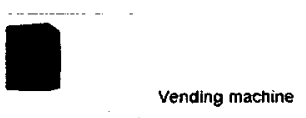

\section{Question 9}

From memory, in a straight line distance, out of a choice of three which object is closer to the Road sign?

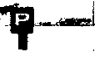

A

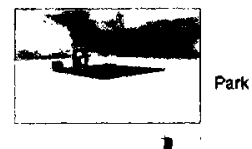

B. I 11,1

c.

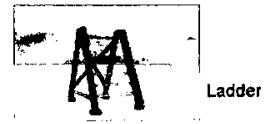

Question 10

From memony, in a straight line distance, out of a choice of three which object is closer to the Bus?

iis.

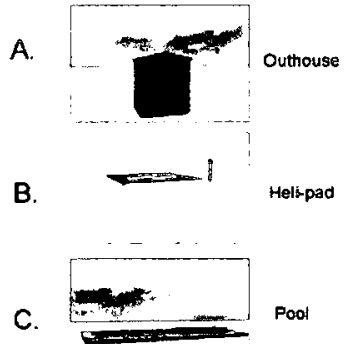

\section{Question 11}

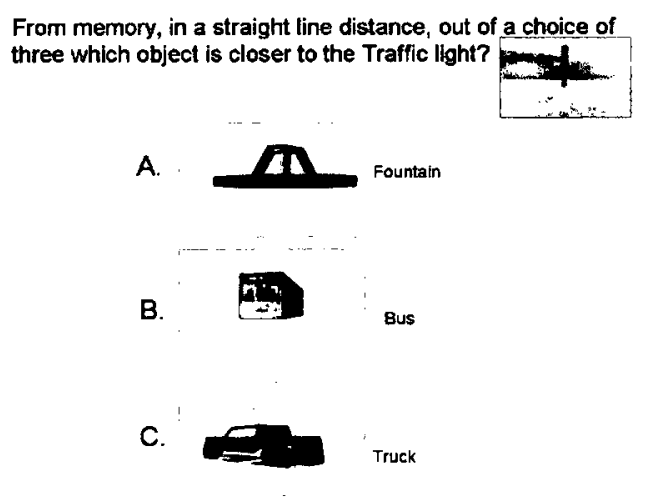

\section{Question 12}

From memory, in a straight line distance, out of a choice of three which object is closer to the Heli-pad? $\rightarrow-i$

A

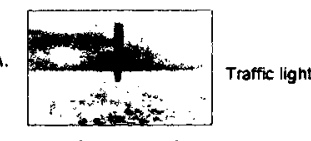

B.

Fountain

c.

Vending machine 
5. JRD and RDE Results

\section{Experiment 1: JRD Performance.}

The mean absolute deviation of the direction indicated by the participant from the correct direction (in degrees) was used for all analyses of judgments of relative direction and therefore the lower the number the better the performance. It was hypothesized that groups exposed to global landmarks would outperform those who were not exposed to them on judging direction. A planned comparison independent t-test was used to investigate differences between Global and Local-only performance on judgment of relative direction. This test revealed that there were no significant differences found between Global-only and Local-only groups in JRD performance.

Differences between the Global and Local-only groups based on the location of landmarks were investigated. It was hypothesized that the Global-only group would outperform the Local-only group at both landmark locations. Performance did not significantly differ based on the landmark locations.

JRD Performance by Size and Location. The Both group was included in separate analyses investigating the differences that may exist between JRD performance based on the type and location of landmarks. It was hypothesized that performance in JRD questions involving global landmarks, regardless of their location, would result in better performance when compared to local landmarks. Planned independent $t$-tests revealed that JRD questions involving global landmarks located internally resulted in significantly 
better performance when compared to local landmarks located internally, $t(19)=-2.491$, $\mathrm{p}<.05$, (see Figure 52).

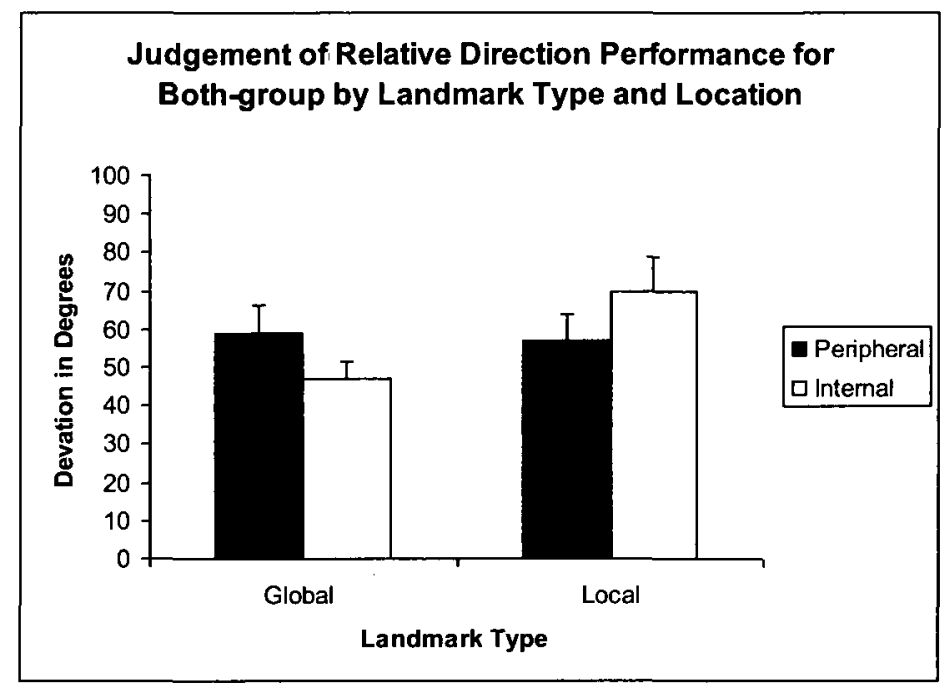

Figure 52. Orientation deviation in degrees (+SE) for Both group by landmark type and location.

JRD Performance with Global Landmarks by Location. The Both and Globalonly groups were included in an analysis to further investigate the impact of global landmark location on judgement of direction. Differences found between these two groups can be attributed to the impact that the absence or presence local landmarks may have on judgment of direction. Only those questions referring to global landmarks were included in this analysis, i.e. all 12 JRD questions from the Global-only group and a subset of 6 of the $12 \mathrm{JRD}$ questions from the Both group. It was hypothesized that global landmarks located in the periphery would result in better performance when judging direction as they are to provide compass-like information. No differences were found as revealed by planned independent t-tests.

JRD Performance for Local Landmarks by Location. The Both and Local-only 
groups were included in this analysis to investigate the impact of local landmark location on judgment of direction. Differences found between these two groups can be attributed to the impact that the absence or presence global landmarks may have on judgment of direction. Only those questions referring to local landmarks were included in this analysis, i.e. all 12 JRD questions from the Local-only group and a subset of 6 of the 12 JRD questions from the Both group. It was hypothesized that the Both-group would outperform the Loca-only group as they have access to global landmarks which are to provide information enabling survey knowledge acquisition. No differences were found as revealed by planned independent t-tests.

\section{Experiment 1: RDE Performance}

Estimating straight line distances between landmarks can reflect participants' survey knowledge as contextual egocentric information was not provided within the questions. It was hypothesized that groups exposed to global landmarks would outperform those who received local landmarks only. The mean of correct responses was used to test for differences in all analyses referring to relative distance estimation (RDE). Differences between those exposed to only global and local landmarks analyzed by planned comparison independent t-test which revealed no significant differences.

Planned independent t-tests were used to test Global-only and Local-only group differences as a function of landmark locations. It was hypothesized that regardless of location, the global-only group would result in better performance. No between group differences were found. Planned paired sample t-tests were used to investigate landmark 
location differences within groups which revealed significantly better performance for local landmarks located internally, $\mathrm{t}(19)=-2.162, \mathrm{p}<.05$ (see Figure 50).

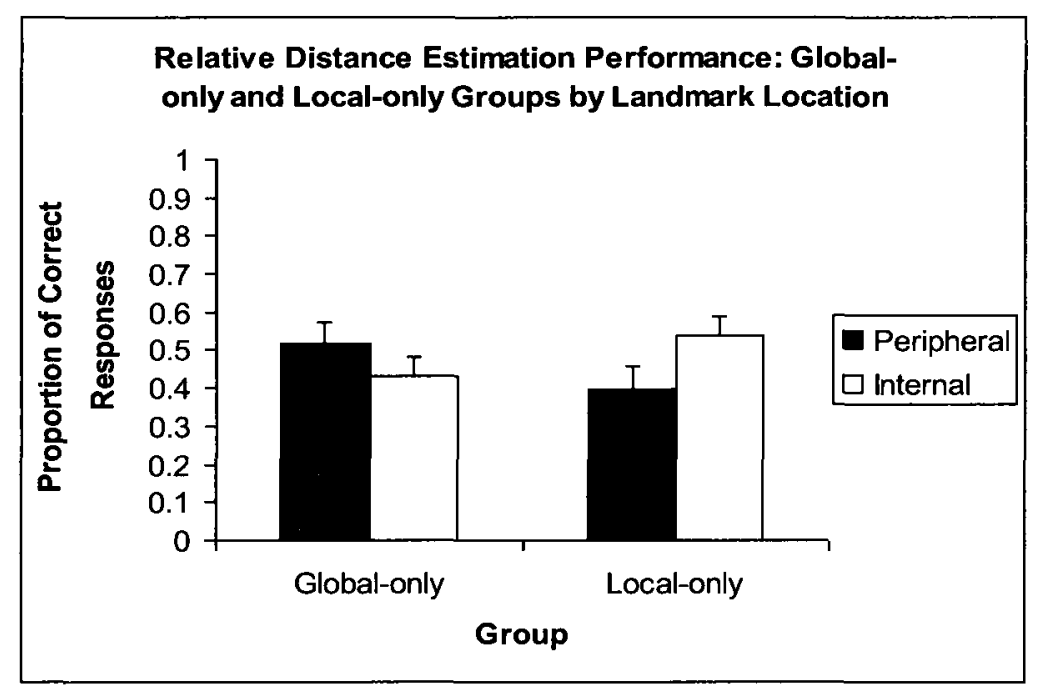

Figure 50. Relative distance estimation performance (+SE) for Global and Localonly groups by landmark location.

RDE Performance by Type and Location. The Both group was included in separate analyses investigating the differences that may exist between RDE performance based on the type and location of landmarks. It was hypothesized that performance for judging direction would be significantly better when referring to global landmarks as they are hypothesized to be associated with direction information. Planned comparison paired sample t-tests revealed that performance for $\mathrm{RDE}$ questions referring to global landmarks located in the periphery resulted in significantly better performance when compared to those located internally, $t(19)=-2.486, p<.05$ (see Figure 51). 


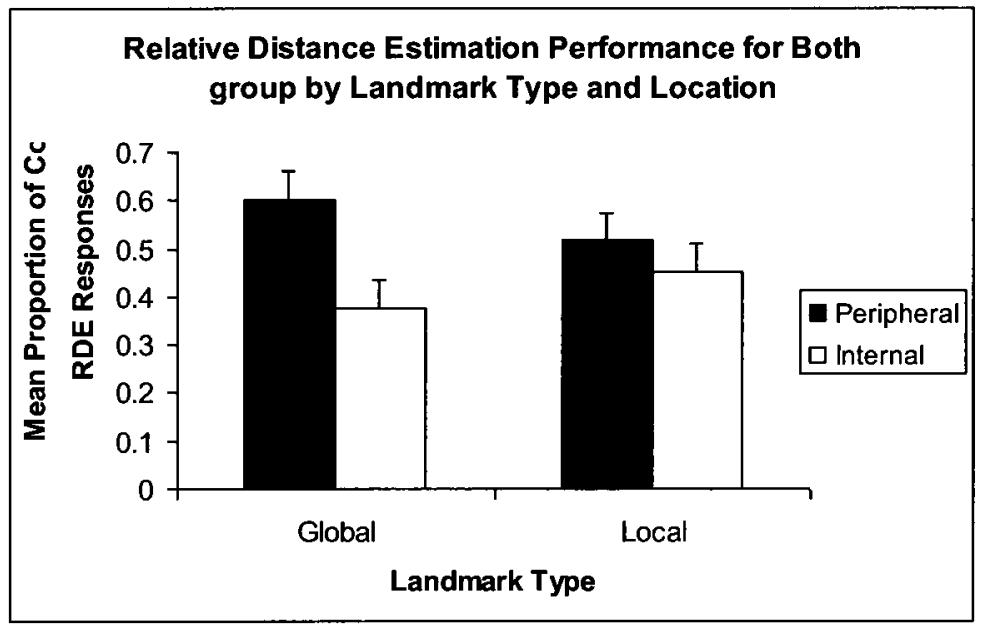

Figure 51. Relative distance estimation performance $(+\mathrm{SE})$ for Both group by landmark size and location.

RDE Performance for Global Landmarks by Location. The Both and Global-only groups were included in this analysis to investigate the impact of global landmark location on RDE performance. Differences found between these two groups can be attributed to the impact that the absence or presence that local landmarks has on RDE performance. Only those questions referring to global landmarks were included in this analysis, i.e. all 12 RDE questions from the Global-only group and a subset of 6 of the 12 RDE questions from the Both group. Planned independent t-tests revealed no differences between groups at either landmark location.

RDE Performance for Local Landmarks by Location. The Both and Local-only groups were included in this analysis investigating the impact of local landmark location on distance estimation accuracy. Differences found between these two groups can be attributed to the impact that the absence or presence that global landmarks has on $\mathrm{RDE}$ performance. Only questions referring to local landmarks were included in this analysis, 
i.e. all $12 \mathrm{RDE}$ questions from the Local-only group and a subset of 6 of the $12 \mathrm{RDE}$ questions from the both group. Planned independent t-tests revealed that the Local-only group performed significantly better than the Both-group with landmarks located internally, $\mathrm{t}(38)=-2.410, \mathrm{p}<.05$ (see Figure 53).

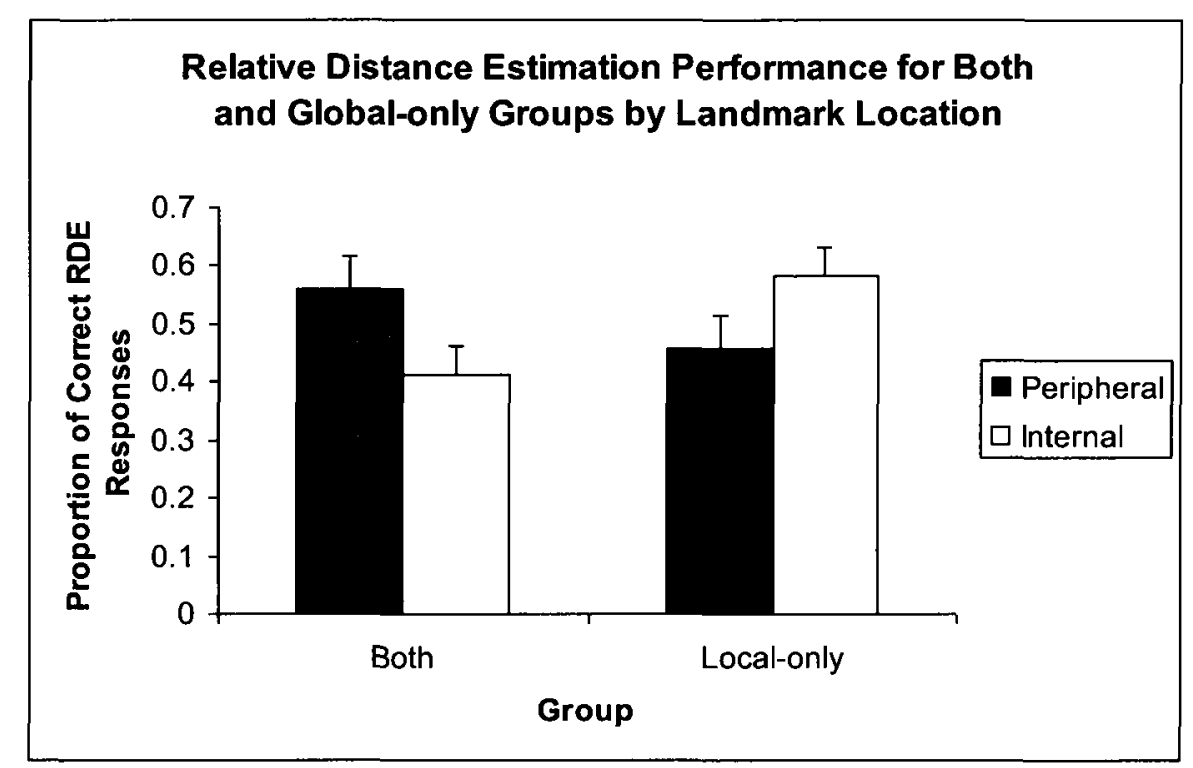

Figure 53. Mean proportion of correct RDE responses (+SE) as a measure of distance performance of Both and Global-only groups for global landmarks by location.

\section{Experiment 2: JRD JRD Performance}

Based on findings from Experiment 1 it was hypothesized that groups exposed to global-internal landmarks, regardless of condition, would perform significantly better than all other groups. The mean absolute deviation between the direction indicated by the participant and the correct direction (in degrees) was used for all analyses; i.e. the lower the deviation - the better the performance.

JRD Performance by Location at Sequence 1. Planned comparison independent t- 
tests were used to investigate differences between peripheral and internal-only group performance on judgment of relative direction at sequence 1, i.e. when exposed to global followed by local landmarks. No significant differences were found. Paired sample t-tests were used to investigate differences as a function of landmark type within each group. No significant differences were found between global and local landmarks for both locations. JRD Performance by Location at Sequence 2. Planned comparison independent ttests was used to investigate differences between the peripheral and internal-only groups performance on judgment of relative direction at sequence 2, i.e., when exposed to local followed by global landmarks. The hypothesis posed was not supported, and it was found that those exposed to peripheral landmarks performed significantly better when exposed to local landmarks, $\mathrm{t}(33.721)=-2.417, \mathrm{p}<.05$ (see Figure 54).

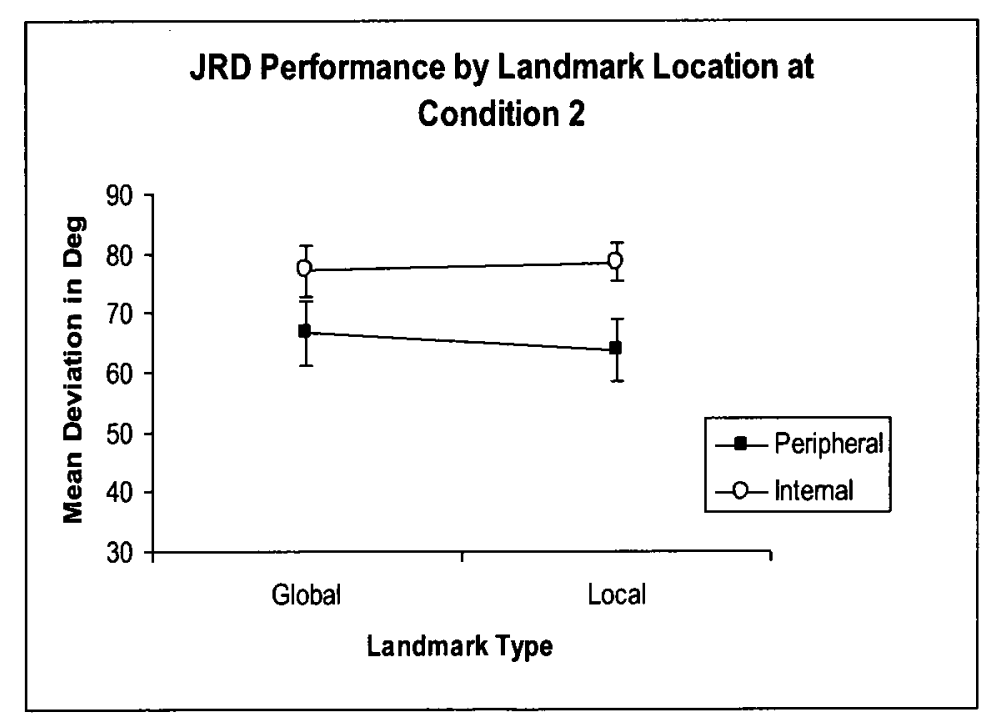

Figure 54. JRD performance as measured by mean deviation in degrees (+SE) as a function of landmark location at sequence 2 . 
Paired sample t-tests were used to investigate differences of landmark type within each group. No significant differences were found between global and local landmarks for both locations.

\section{Experiment 2: RDE Performance}

Knowledge used to estimate straight line distances between landmarks an reflect participants' survey knowledge as contextual information was not provided. The literature reviewed suggests that global landmarks situated on the periphery aid in survey related knowledge acquisition. Consequently, it was hypothesized that groups exposed to global-peripheral landmarks would perform better than those exposed to internal landmarks, regardless of type. The mean of correct responses of relative distance estimation (RDE) was used to test the hypothesized differences.

RDE Performance by Location at Order 1. Planned comparison independent t-test was used to test RDE performance differences between those exposed to only peripheral and those exposed to only internal landmarks. No differences were found between the groups. Paired sample t-tests also revealed no differences between phases within groups. RDE Performance by Location at Order 2. Planned comparison independent t-test was used to test RDE performance differences between those exposed to only peripheral and those exposed to only internal landmarks. No differences were found between the groups. Paired sample t-tests also revealed no differences between phases within groups. 


\section{Experiment 3: JRD Performance}

Planned comparison paired sample and independent $t$-tests were performed to test for differences of JRD performance within and across the three levels of experience tested in this experiment. These analyses revealed what types of landmarks are associated with judging direction as a function of experience. Within groups paired sample t-tests provided information regarding differences between global and local landmark acquisition in reference to judging directions at each level of experience. The between groups t-tests provided information regarding the global and local landmark acquisition between levels of experience.

Based on the literature review and previous findings, it was hypothesized that performance will improve with experience, and that performance involving global landmarks will be superior to performance involving local, regardless of experience. Independent sample t-tests revealed a significant improvement when judging direction involving global landmarks between the first and second blocks, $t(36)=2.222, \mathrm{p}<.05$, but did not show any further improvement following the second block. A significant improvement over time when judging distances to local landmarks between the first and last block, $\mathrm{t}(35)=2.935, \mathrm{p}<.05$ was found. Differences within the group tested following block 2 were significantly better at judging direction between global landmarks compared to local ones, $\mathrm{t}(18)=-2.439, \mathrm{p}<.05$. 


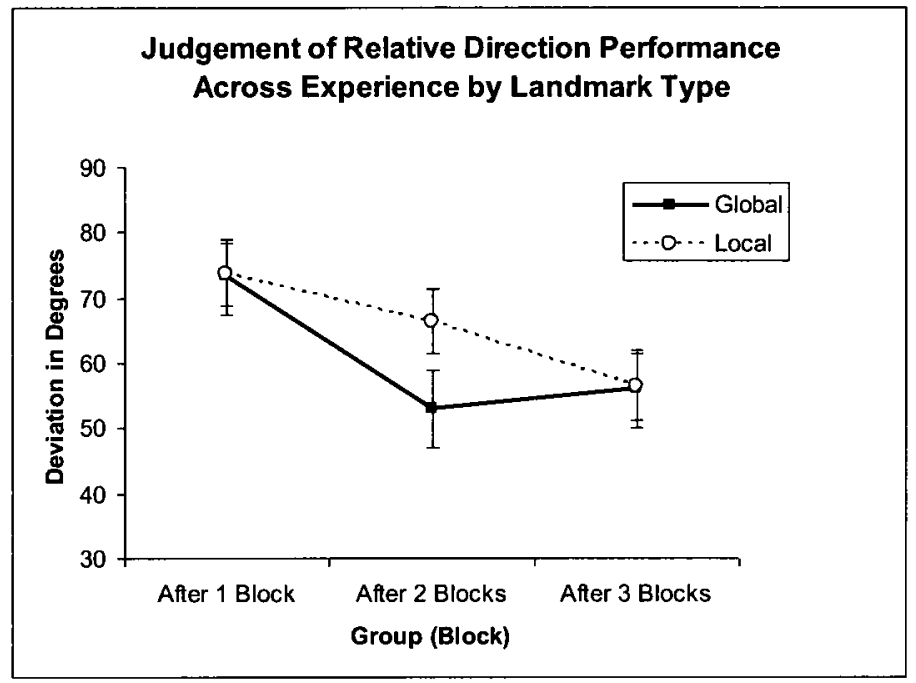

Figure 55. Orientation deviation in degrees ( $+\mathrm{SE})$ by landmark type across level of experience.

A separate analysis was completed to investigate the hypotheses that performance will improve with experience, and that performance involving global landmarks will be superior to performance involving local, regardless of experience. Paired sample t-tests revealed that performance was significantly better when judging direction to global landmarks in the last block, $\mathrm{t}(19)=-2.099, \mathrm{p}<.05$. 


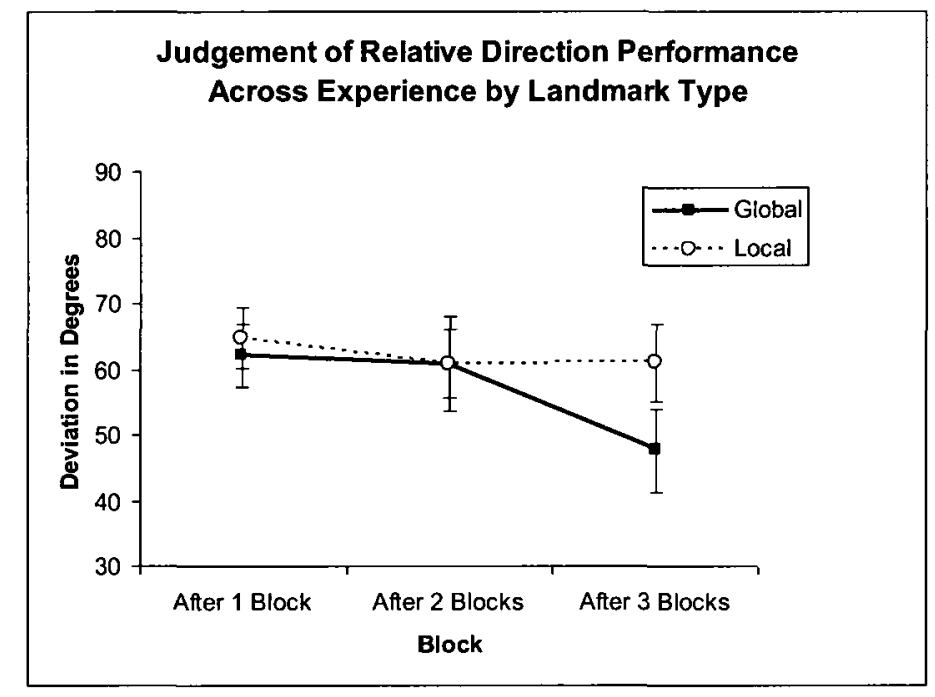

Figure 56. Orientation deviation in degrees (+SE) by landmark type across level of experience for "All blocks" group.

JRD Performance as a Function of Landmark Location across Experience. To investigate the effect different landmark locations (peripheral/internal) have as a function of experience, planned comparison paired sample and independent t-tests were performed to test for differences within and across the three levels of experience. These analyses revealed what landmark locations are acquired to judge direction across time. Within groups paired sample t-tests provided information regarding differences between peripheral and internal landmark acquisition in reference to judging directions at each level of experience. The between groups t-tests provided information regarding the peripheral and internal landmark acquisition between all levels of experience.

Given the results of Experiment 1 for the Both group, it was hypothesized that performance would be better on questions related to internal landmarks regardless of experience. Independent sample t-tests revealed a significant improvement when judging 
direction involving internal landmarks between the first and second blocks, $t(37)=2.068$, $\mathrm{p}<.05$, but did not show any further improvement following the second block (see Figure 57). Between the first and last block (i.e. overall) there was a significant improvement when judging direction between internal landmarks $t(37)=2.787, \mathrm{p}<.05$, but not between peripheral ones. Planned comparisons independent $t$-tests indicated that there were no significant differences in judging direction between peripheral and internal landmarks at each level of experience (see Figure 57).

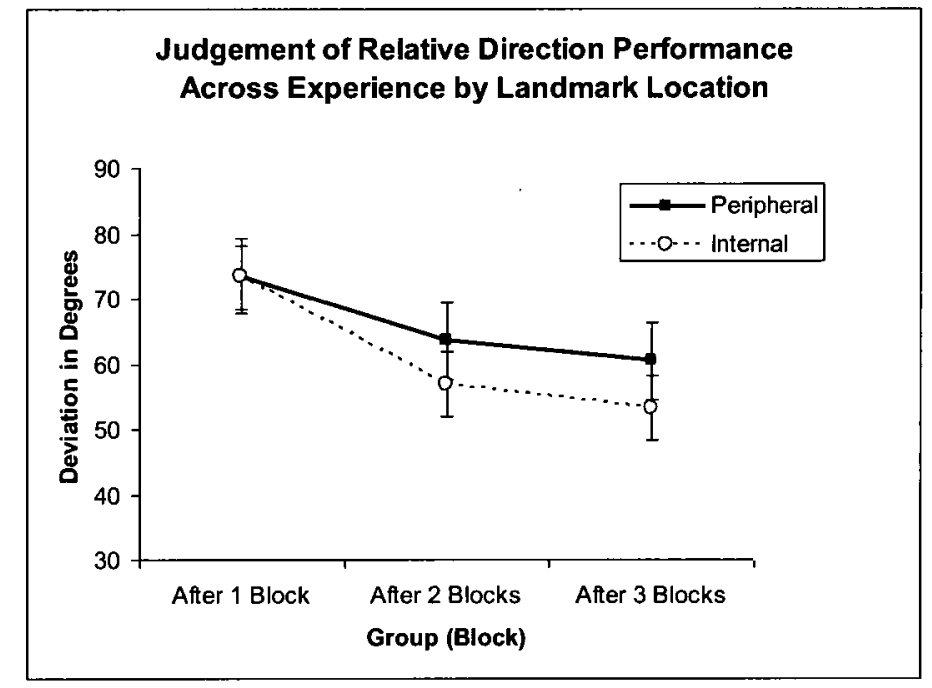

Figure 57. Orientation deviation in degrees (+SE) by landmark location across level of experience.

A separate analysis was conducted to investigate the hypothesis that performance would be better on questions related to internal landmarks regardless of experience for the "After all Blocks" control group. Differences found within this group could be the result of either the variable of interest or the effect of repeated testing. Planned paired 
sample t-tests revealed that participants performed better when judging direction to internal landmarks in the first block only, $t(19)=2.237, \mathrm{p}<.05$.

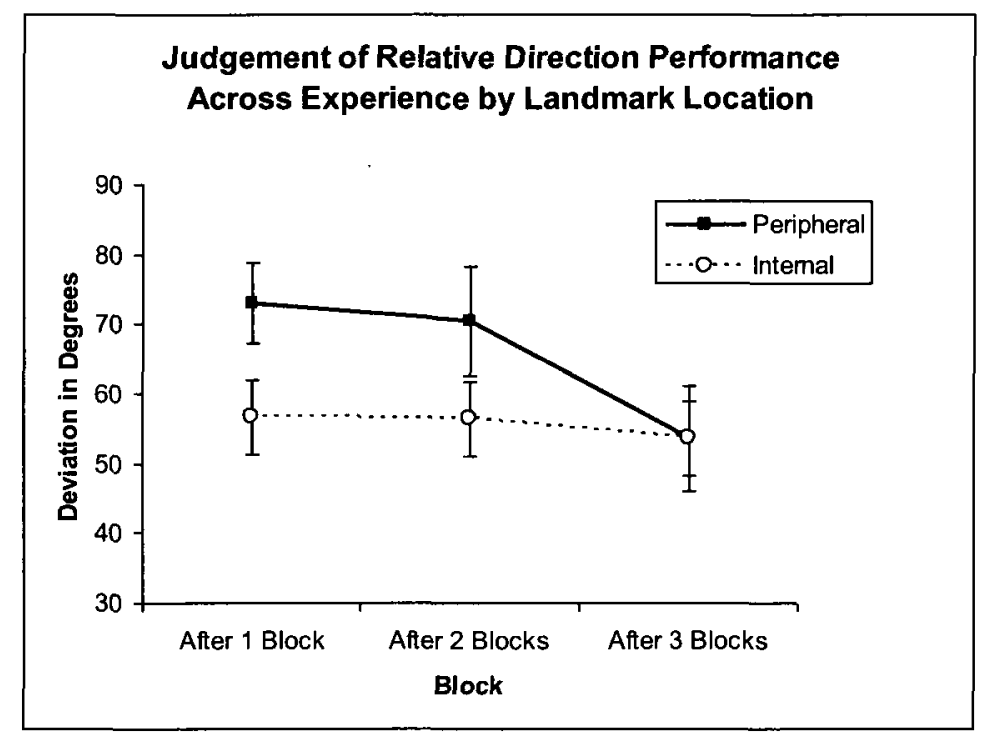

Figure 58. Orientation deviation in degrees (+SE) by landmark location across level of experience for "All blocks" group.

\section{JRD Performance as a Function of Landmark Location and Type across}

Experience. Planned comparison paired sample t-tests were performed to test for differences of JRD performance across the three levels of experience as a function landmark type and location. These analyses revealed the combination of landmark types and locations are acquired to judge direction.

Given the findings from Experiment 1, it was hypothesized that performance would be best when answering questions relating to landmarks classified as globalinternal regardless of experience. Independent sample t-tests revealed a significant improvement for global-internal landmarks between blocks 1 and $2, t(37)=2.074, p<.05$. Paired sample $t$-tests revealed that regardless of experience, participants were better when 
referring to global internal landmarks compared to global peripheral, $\mathrm{t}(57)=3.502$, $\mathrm{p}<.001$ and local peripheral landmarks, $\mathrm{t}(57)=2.118, \mathrm{p}<.05$, and local internal, $\mathrm{t}(57)=$ $3.354, \mathrm{p}<.001$. In addition paired sample $\mathrm{t}$-tests revealed that regardless of experience, participants were better when referring to local peripheral landmarks compared to global peripheral, $\mathrm{t}(57)=2.118, \mathrm{p}<.05$.

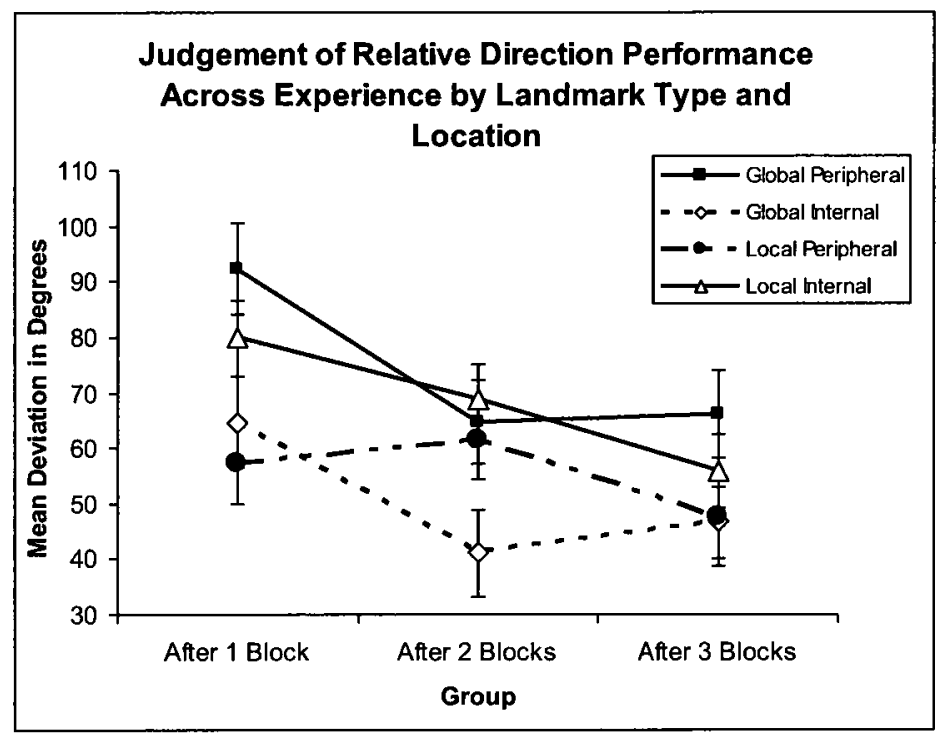

Figure 59. JRD performance by landmark type and location across experience.

A separate analysis was conducted to investigate the hypothesis that performance would be better on questions related to global internal landmarks regardless of experience for the "After all Blocks" control group. Again, differences found within this group could be the result of either the variable of interest or the effect of repeated testing. No significant differences were found within each classification of landmark across the various levels of experience. 


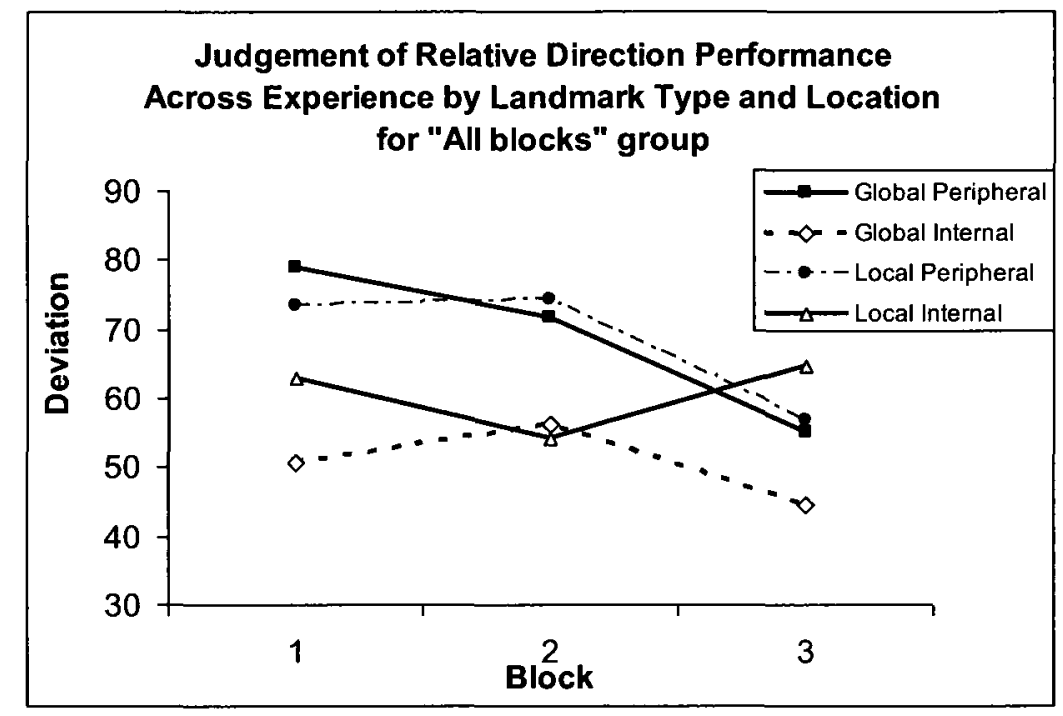

Figure 60. JRD performance as a function of landmark type and location across experience for the control group.

\section{Experiment 3: RDE Performance}

RDE Performance by Landmark Type across Experience. Planned comparison paired sample and independent t-tests were performed to test differences in RDE performance within and across the three levels of experience. These analyses revealed what types of landmarks are acquired to judge straight-line distance as a function of experience. Within groups paired sample t-tests provided information regarding differences between global and local landmark acquisition in reference to judging distance at each level of experience, and the between groups $\mathrm{t}$-tests provided information regarding the global and local landmark acquisition between all levels of experience.

It was hypothesized that judging distances between global landmarks would result in better performance regardless of experience level. There was a significant performance improvement between blocks 1 and 2 in judging distances to local landmarks, $t(37)=-$ 
$2.599, \mathrm{p}<.05$, but not to global. No other significant differences were found across levels of experience. In addition, no differences between landmark types were found within blocks (seeFigure 61).

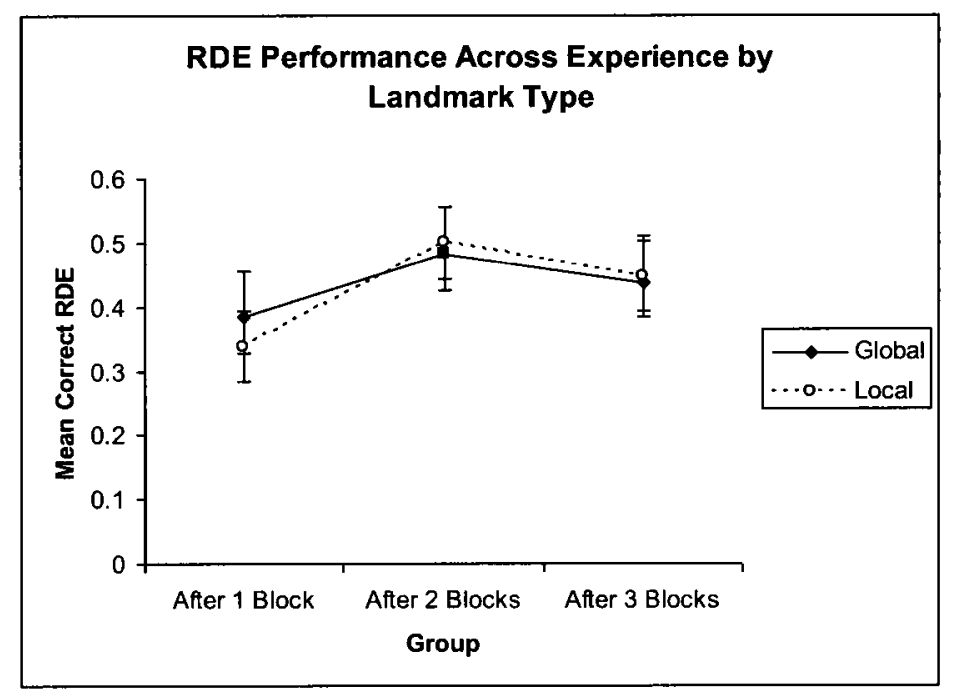

Figure 61. RDE performance (+SE) by landmark type across level of experience.

A separate analysis was conducted to investigate the hypothesis that no difference exist between global and local landmarks when judging distance for the Control group. No differences across or within blocks were found to differ for the control group (see Figure 62). 


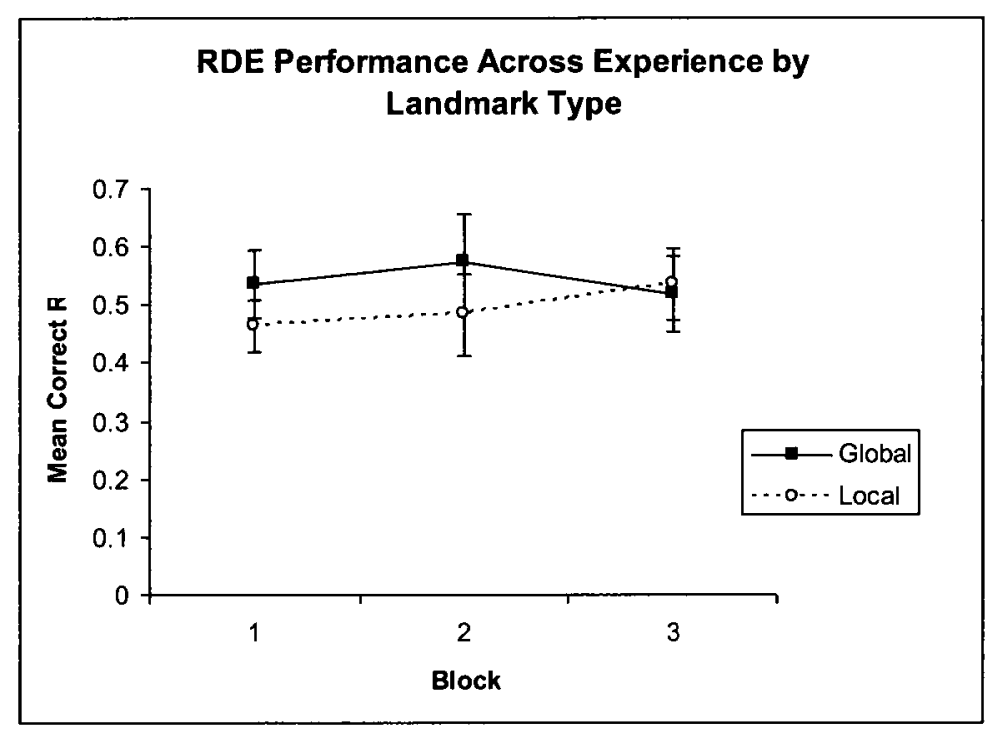

Figure 62. RDE performance $(+\mathrm{SE})$ by landmark type across level of experience for the control group.

RDE Performance as a Function of Landmark Location and Experience. Planned comparison paired sample and independent t-tests were performed to test for differences of RDE performance within and across the three levels of experience tested in this experiment. These analyses revealed what landmark locations are acquired to judge straight-line distance as a function of experience. Within groups paired sample t-tests provided information regarding differences between peripheral and internal landmark acquisition in judging distance at each level of experience, and the between groups t-tests provided information regarding the peripheral and internal landmark acquisition between all levels of experience.

Given the results of Experiment 1, it was hypothesized that there would be no significant differences in judging distances between landmarks based on their location. No differences between landmark-locations were found within blocks (see Figure 63). 
With respect to experience levels, it was found that there was a significant improvement in performance between blocks 1 and 2 when judging distances to peripheral landmarks, $t(37)=-2.260, p<.05$. No other significant differences were found across levels of experience.

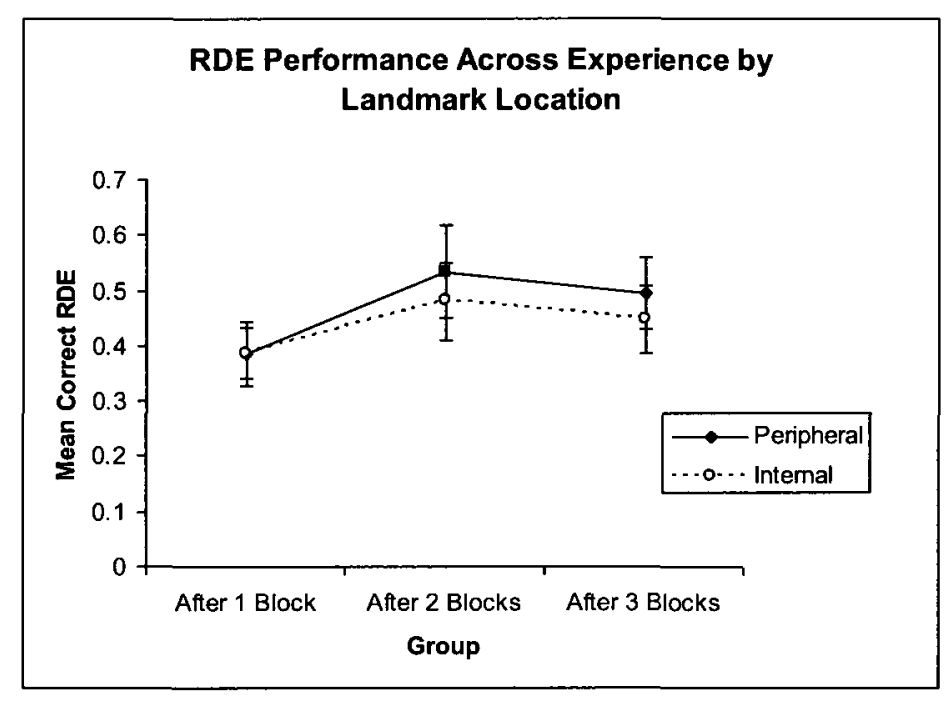

Figure 63. RDE performance (+SE) by landmark location across level of experience.

A separate analysis was conducted to investigate the hypothesis that no difference exist between global and local landmarks when judging distance for the Control group. No differences across or within blocks were found to differ for the control group 


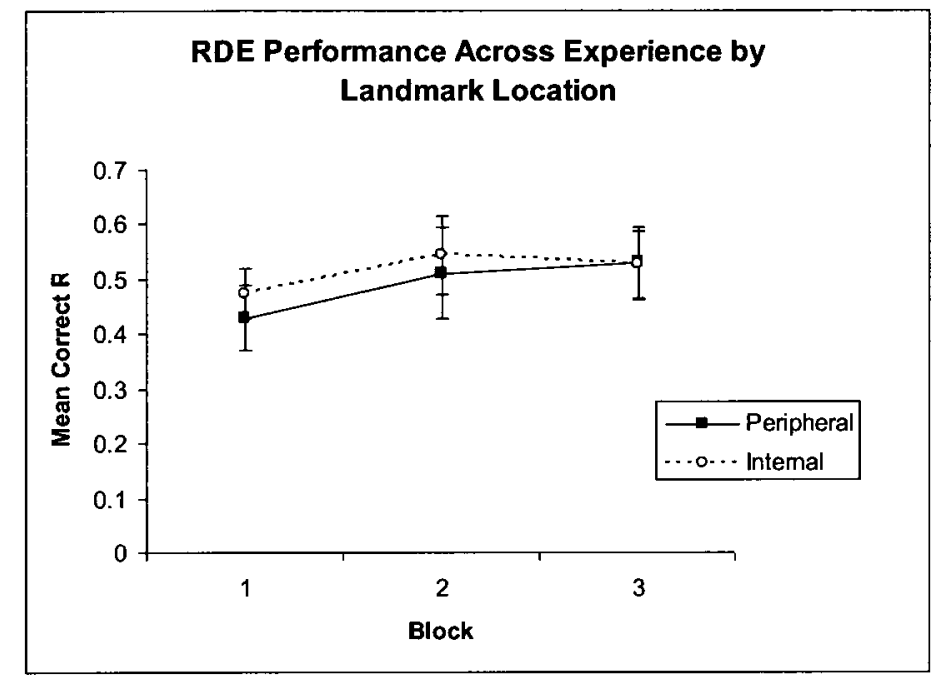

Figure 64. RDE performance (+SE) by landmark location across level of experience for the control group.

RDE Performance by Landmark Location and Type as a Function of Experience. Planned comparison paired sample and independent $t$-tests were performed to test for differences of RDE performance within and across the three levels of experience tested in this experiment for landmark type and location. These analyses reveal what combination of landmark types and locations are acquired to judge straight-line distance across time.

A paired sample t-test revealed that RDE performance was significantly better when referring to global peripheral landmarks compared to global internal, $t(57)=2.747$, $\mathrm{p}<.05$ and local peripheral landmarks, $\mathrm{t}(37)=2.588, \mathrm{p}<.05$ but not local internal. 


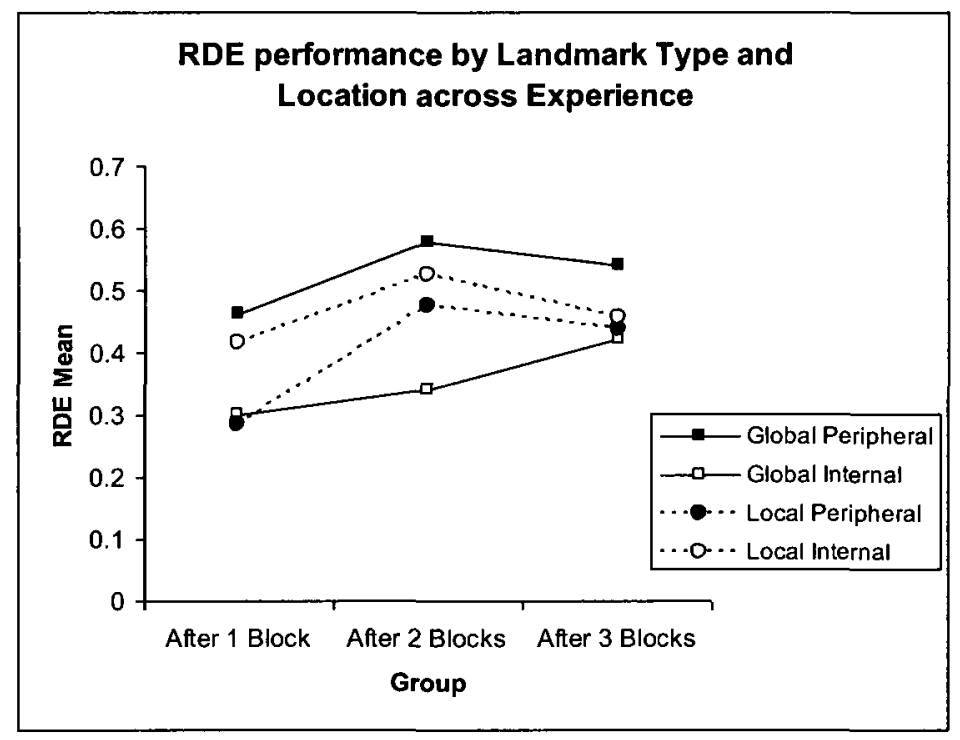

Figure 65. RDE performance by landmark type and location across experience.

A separate analysis was conducted to investigate the hypothesis that no difference exist between global and local landmarks when judging distance for the Control group. No significant differences were found between the landmarks based on type and location for the control group. 


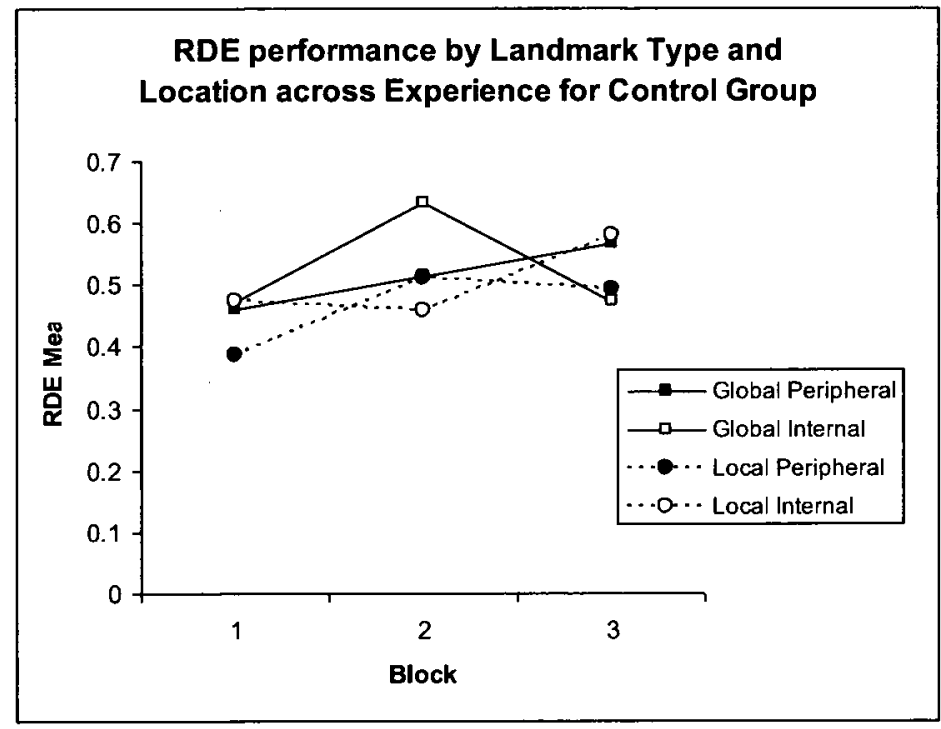

Figure 66. RDE performance by landmark type and location across experience for control group. 
Appendix F. Informed Consent Form

\section{Landmarks and Navigating 3D Virtual Environments}

You are being asked to participate in a study designed to assess how easy it is to navigate in virtual reality spaces displayed on a desktop computer while using a map. The information in this form is intended to help you understand exactly what we are asking of you so that you can decide whether or not you want to participate in this study. Please read this consent form carefully and ask all the questions you might have before deciding whether or not to participate or not in this study. Your participation in this study is entirely voluntary, and a decision not to participate will not in any way be used against you.

\section{Project Team}

\begin{tabular}{|c|c|}
\hline Avi Parush, $\mathrm{PhD}$ & Shelley Roberts, B.A, M.S \\
\hline Associate Professor & Graduate Student \\
\hline Department of Psychology & Department of Psychology \\
\hline $\begin{array}{l}\text { Carleton University } \\
\text { aviparush@carleton.ca }\end{array}$ & $\begin{array}{l}\text { Carleton University } \\
\text { smrobert } @ \text { carleton.ca }\end{array}$ \\
\hline $613-520-2600 \times .6026$ & $613-520-2600 \times .6628$ \\
\hline
\end{tabular}

\section{Purpose of the Study}

You are invited to participate in research into navigation in virtual environments. People often become lost and disoriented when navigating in unfamiliar environments. One way to improve the navigability of spaces is to first understand how people navigate, and then use this understanding to improve the design of space and/or to develop navigational aids.

In this study, you will navigate in virtual environments presented on a desktop computer screen. Virtual environments provide an important advantage over real environments in the study of navigation: virtual environments are much easier to modify than real ones.

\section{What Will You Be Asked To Do?}

The experiment consists of 5 parts. The first part of the experiment consists in filling out two short questionnaires. The second part involves navigating in a virtual environment using a map. In this part, you will begin with a practice session during which you will familiarize yourself with how to navigate in a virtual environment. While navigating, you will be asked to find targets in the virtual environment as quickly as possible. Once you have completed navigation, you will be asked questions about the 
direction in which other objects in the virtual environment are located and how far away they are. The computer will record your answers and movements through the environments during this part of the experiment. Feel free to ask questions about your experience and to omit any answers you feel uncomfortable with.

\section{The whole experiment should take about 1.5-2.0 hours to complete.}

Note that this experiment is not intended to assess your ability to interact with a computer or your general ability to navigate. Rather, it's the environment and interface that are being tested for the ease of use.

\section{Benefits}

Many of the people participating in these types of experiments have enjoyed their experience. Moreover, your participation will contribute to our effort to make interaction with 3D computer environments easier and more effective.

\section{Risks}

With virtual reality, there is always a risk of cybersickness or VR sickness. Cybersickness has three major components: nausea, eye discomfort, and dizziness.

In this experiment, you will be interacting with virtual environments through a desktop computer. This substantially reduces the risks of cybersickness compared to using large displays or head-mounted displays. Consequently, you are unlikely to suffer from any of the symptoms mentioned above. However, if you do feel the onset of one of these symptoms, sit still and close your eyes for 10 seconds. This technique should completely eliminate any symptom. If symptoms persist, you should notify the experimenter who will stop the experiment. (Note that you will still be given an experimental credit or payment.) Note that if you have played 3D computer games without feeling "cybersick", you are very unlikely to feel any symptoms during this experiment. However, if you are not in your normal state of health, you should not participate. Being overtired, taking medication, having the flu, or other such states increase the risk of cybersickness.

\section{Privacy and Confidentiality}

Confidentiality will be respected. Unless required by law, no information that might directly or indirectly reveal your identity will be released or published without your specific consent to the disclosure. All participants have the right to obtain any publicly available documents that are published about this research. All data collected is stored securely at the National Research Council of Canada and Carleton University. If you have any questions regarding the experiment and your participation in it, please feel free to ask. 


\section{Payment for Participation}

You will be provided between 1.5 and 2.0 experimental credits. If you wish to stop the session and withdraw from the experiment, you will still receive the compensation.

\section{Withdrawal}

I understand that the investigator can end my participation in the experimental protocol for financial, scientific, or ethical reasons at any time. I also understand that I may end my participation in the experiment at any time or for any reason, without penalty.

\section{Contact Information}

If you have any questions about the experiment, or your participation please ask your experimenter. If you have additional questions, comments or concerns, please contact:

Shelley Roberts, B.A, M.S., Ph.D. candidate

Department of Psychology, HotLab

Carleton University

1125 Colonel By Dr., Ottawa, ON, K1S 5B6

Social Sciences Research Building

Telephone: 613-520-2600 X6628

\section{Statement of Consent - Signatures}

$\mathrm{I}$, , have read, or have been read, and understand the information given in this informed consent and all my questions have been answered to my satisfaction. I have had sufficient time to consider whether to participate in this study. I understand that my participation in this study is entirely voluntary and that I may withdraw from the study at any time without penalty.

I voluntarily consent to participate in this study. I also certify that I am in my normal state of health.

Participant signature 
To the best of my knowledge, the information in this consent form, and the information that I have provided in the response to any questions, fairly represents the project. I am committed to conducting this study in compliance with all the ethical standards that apply to projects that involve human participants. I will ensure that the participant receives a copy of this consent form.

Experimenter signature 


\section{Appendix G. Demographics Questionnaire}

\section{Demographics Questionnaire}

1. Gender: $\quad \mathrm{M} \quad \mathrm{F}$

2. Age:

3. Please rate your fluency in English (1=poor, 5=fluent)

$\begin{array}{llllll}\text { Reading: } & 1 & 2 & 3 & 4 & 5 \\ \text { Writing: } & 1 & 2 & 3 & 4 & 5 \\ \text { Speaking: } & 1 & 2 & 3 & 4 & 5\end{array}$

4. Please circle your current education level and list year of program:

High School
College
Year one BA/BSc
Year two BA/BSc
Year three BA/BSc
Year four BA/BSc
$\mathrm{MA} / \mathrm{MSc}$
$\mathrm{PhD}$

5. Please rate your experience with virtual desktop environments, i.e. games, simulations, information spaces etc. $(1=$ No experience, $5=$ Daily experience $)$.

1 2 3

4 5

a) What type of games? (i.e. PC role playing, PS2 sports etc)

b) What input device do you use most frequently?

mouse keyboard joystick gamepad

6. Is your vision normal or corrected to normal (i.e. you have glasses and are wearing them=corrected to normal?)

7. Are you colour blind? Yes No 
8. Please answer the following questions.

\begin{tabular}{|l|l|l|}
\hline \multicolumn{1}{|c|}{ Statements } & True & False \\
\hline \hline I am ill & & \\
\hline I am tired or lack sleep & & \\
\hline I am under the influence of drugs or alcohol & & \\
\hline I have a hangover & & \\
\hline I have an upset stomach & & \\
\hline I am under unusual psychological stress & & \\
\hline $\begin{array}{l}\text { I am susceptible to cyber-sickness induced by } \\
\text { computer/video games. }\end{array}$ & & \\
\hline I suffer from dizzy spells & & \\
\hline I have had an ear infection recently & & \\
\hline I am susceptible to motion sickness & & \\
\hline
\end{tabular}

If you answered true to one or more statements above please notify the experimenter.

9. Have you had any experience with orienteering (cross-country race in which competitors use a map and compass to find their way through unfamiliar territory)?

Yes No

Please specify: 


\section{Appendix H. Experiment Instructions}

An example of the set of instructions in the order in which they were presented to participants prior to navigating the VE. The instructions were slightly different for groups only on slide 3 depending on how many trials were completed prior to testing and how many times the group was tested (e.g. in Experiment 3 groups were tested multiple times).

Slide 1:

\section{Your Task}

You will be asked to find a series of 15 targets within a virtual city using the shortest route and as fast as you can.

Press SPACE BAR to continue

Slide 2:

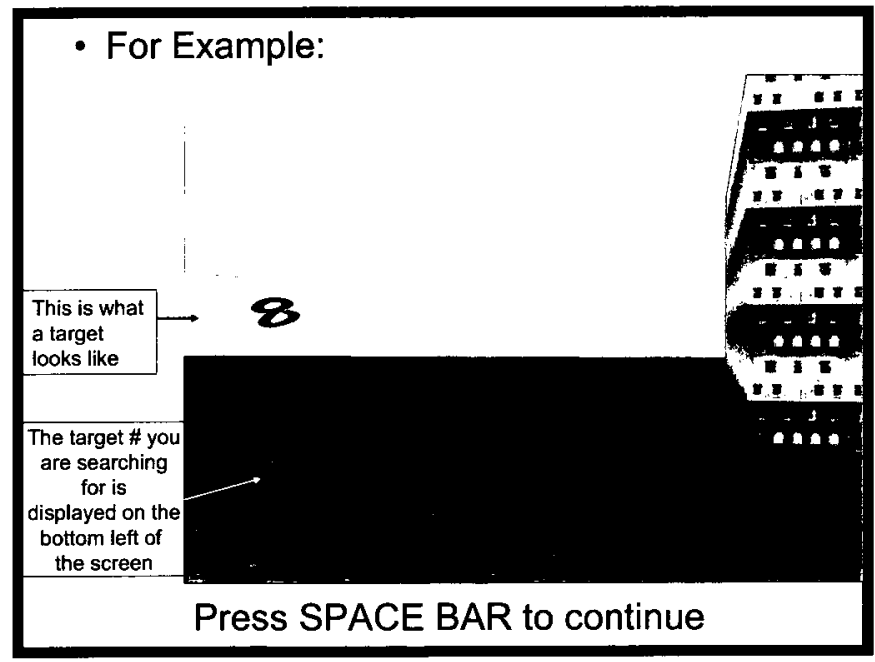


Slide 3:

\section{To Move in the World}

- Use arrow keys to look up and down

- Move mouse left and right to look left and right

- Use left mouse button to move forward

- Use right mouse button to move backwards

Press SPACE BAR to continue

Slide 4:

- After 15 trials, you will be asked:

- a series of questions about the location of objects in the world and their relation to one another and

- to draw a map of the world

- Please keep these tasks in mind as you navigate the world.

- Last but not least...have fun!

Press SPACE BAR to continue 
Slide 5:

\section{Practice}

- Before we begin you will be given a few minutes to get used to navigating the world. 


\section{Appendix I. Individual Target Information}

A List of $\mathrm{x}, \mathrm{y}$ coordinates for each target location, starting position, distance between targets and starting position, orientation angles and optimal number of turns taken to reach each target from its associated starting point.

\begin{tabular}{cccccccc} 
Target & \multicolumn{2}{c}{ Target Position } & \multicolumn{2}{c}{ Start Position } & Angle & $\begin{array}{r}\text { Distance Between } \\
\text { Start and Target }\end{array}$ & \# of turns \\
\hline 1 & $\mathbf{X}$ & $\mathbf{Y}$ & $\mathbf{X}$ & $\mathbf{Y}$ & & & 1 \\
2 & -19 & -49 & 44 & -11 & 0 & 112 & 1 \\
3 & 1 & 42 & 62 & -32 & 24 & 135 & 1 \\
4 & -34 & 37 & -29 & 53 & 264 & 89 & 2 \\
5 & 73 & -51 & 55 & 10 & 168 & 79 & 1 \\
6 & -60 & -7 & 0 & 27 & 48 & 87 & 2 \\
7 & 21 & -22 & 43 & 53 & 72 & 97 & 1 \\
8 & 22 & 5 & 81 & -29 & 192 & 69 & 1 \\
9 & -6 & -31 & -25 & 11 & 216 & 73 & 1 \\
10 & -33 & 11 & -76 & -10 & 336 & 64 & 2 \\
11 & -64 & 34 & 2 & -16 & 96 & 116 & 1 \\
12 & 80 & 26 & 45 & -40 & 144 & 101 & 1 \\
13 & -23 & -12 & -60 & 30 & 240 & 79 & 2 \\
14 & -69 & -32 & -46 & 51 & 120 & 106 & 1 \\
15 & 58 & -31 & 0 & 0 & 288 & 89 & 2 \\
\hline & & & & & & & 1 \\
\hline
\end{tabular}




\section{Appendix J. Way-finding Decision Task (WDT) questions}

A list of all WDT questions received by each group in all three experiments as labeled.

\section{Experiment 1: Both group and Experiment 3: All groups}

\section{Question 1}

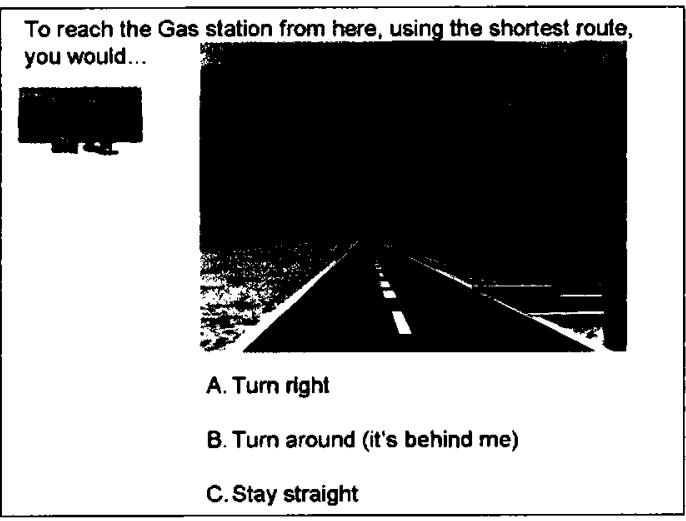

\section{Question 3}

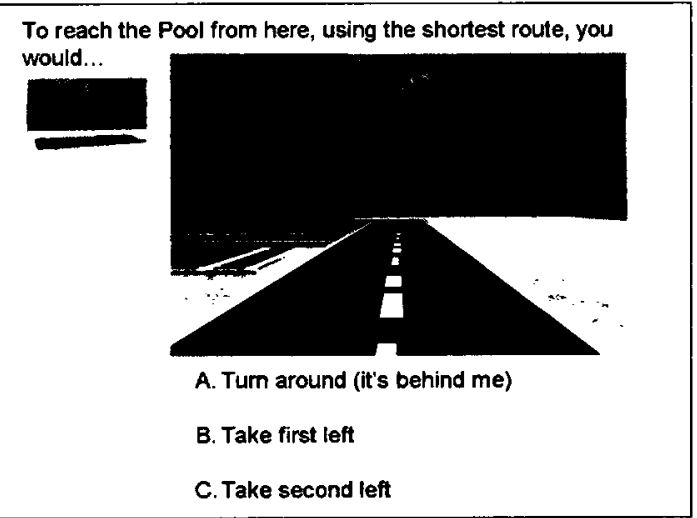

\section{Question 5}

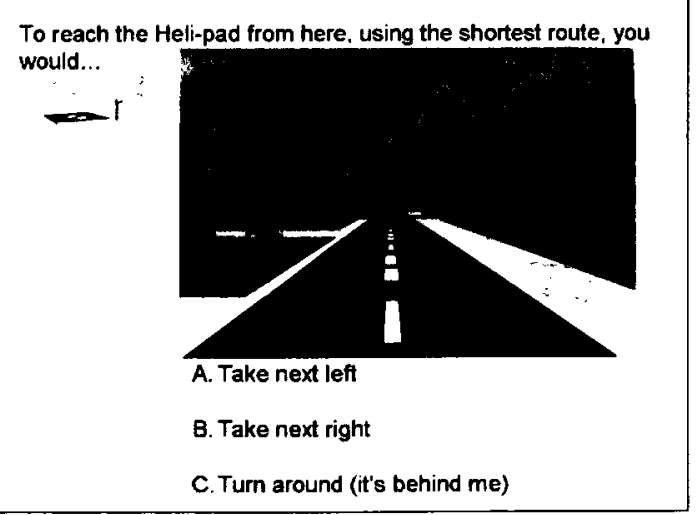

\section{Question 2}

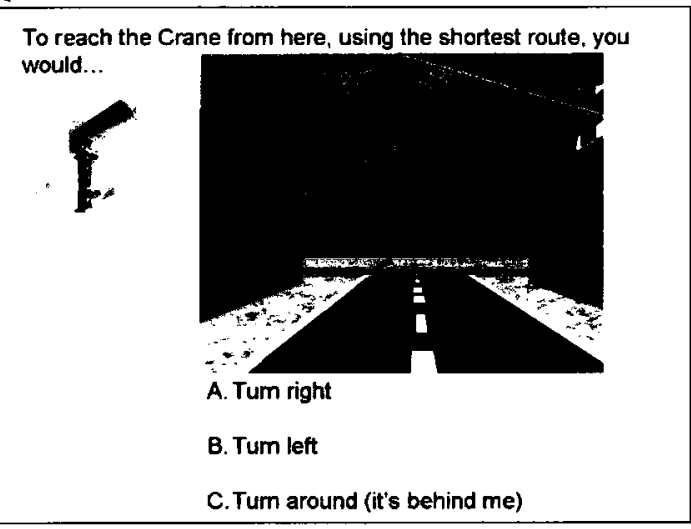

\section{Question 4}

To reach the Garbage bin from here, using the shortest route. you would...

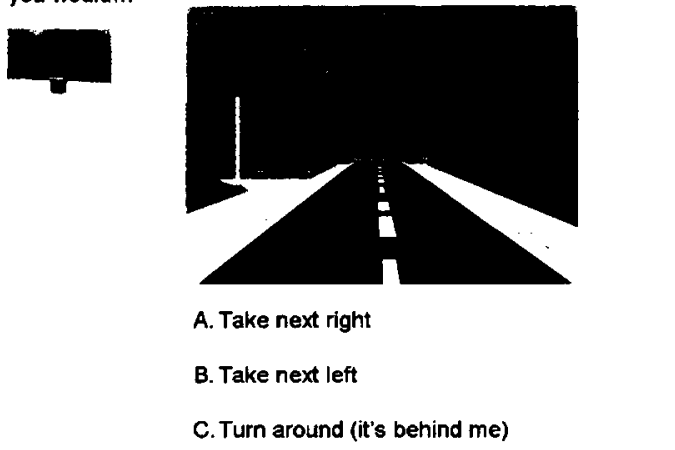

\section{Question 6}

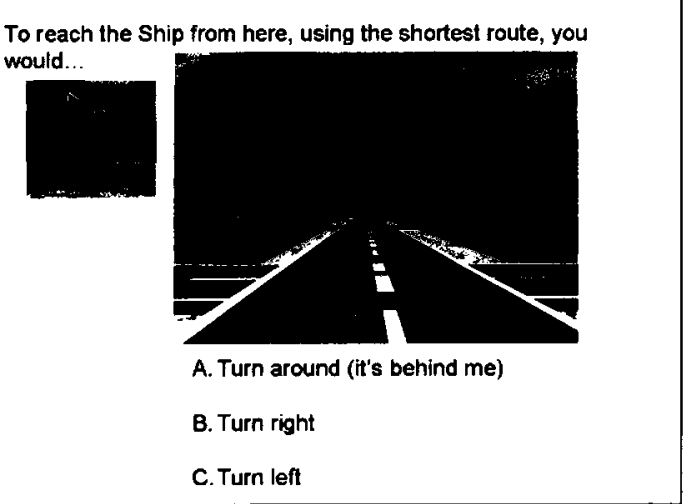




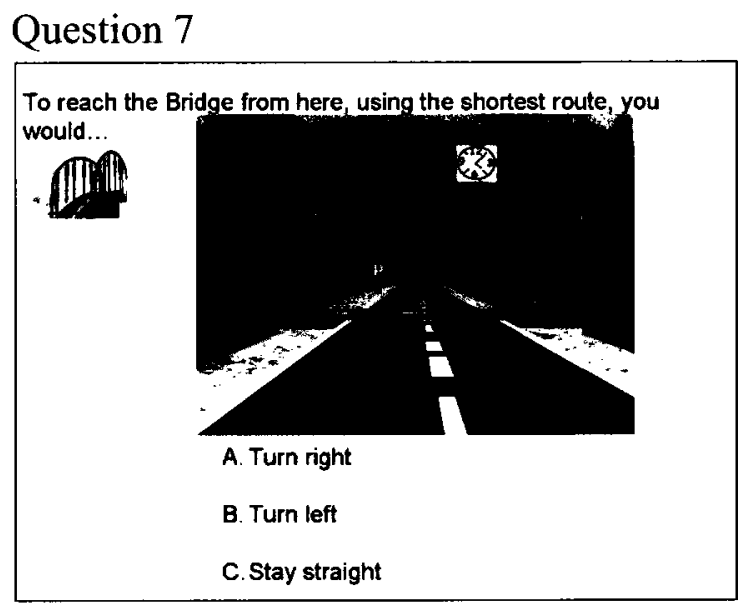

\section{Question 9}

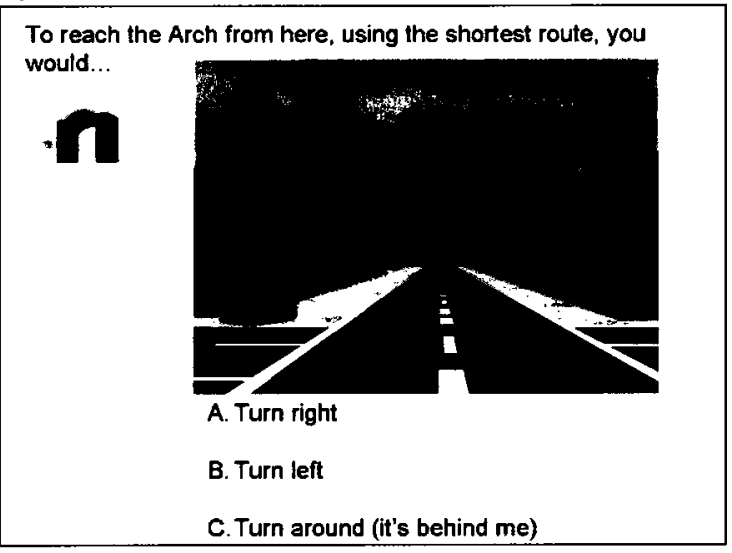

\section{Question 11}

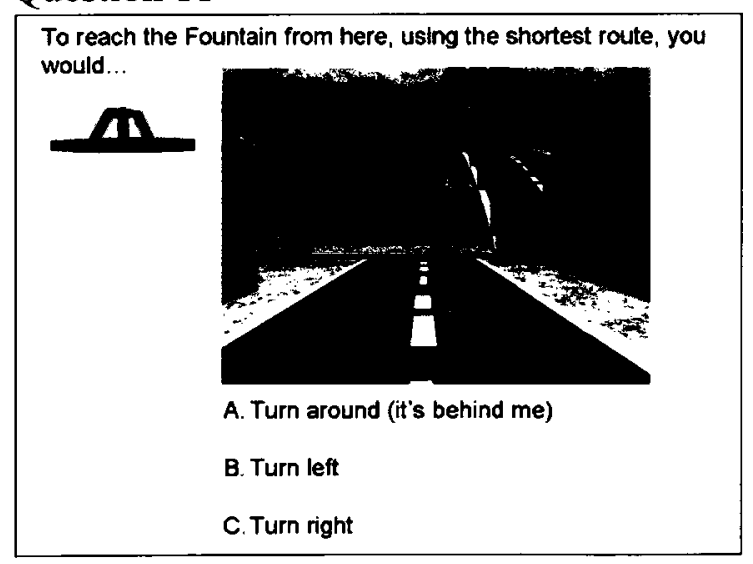

\section{Question 8}

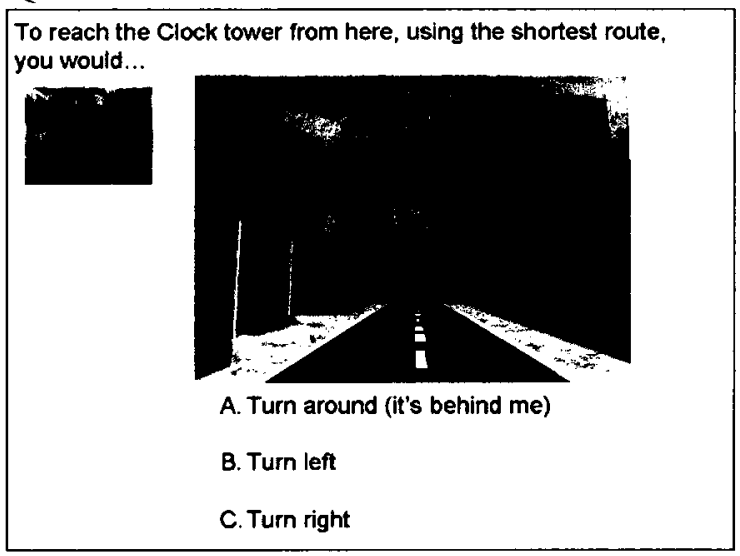

\section{Question 10}

To reach the Mountain from here, using the shortest route, you would...
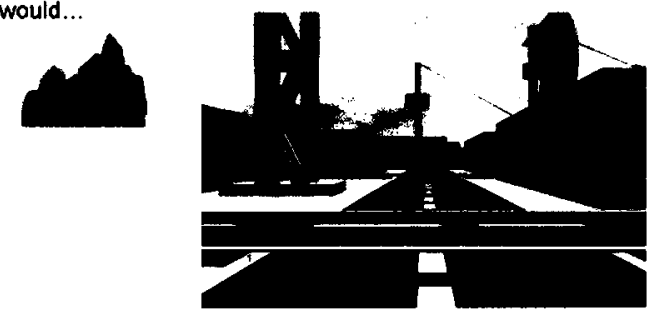
A. Turn left
B. Turn right
C. Turn around (it's behind me)

\section{Question 12}

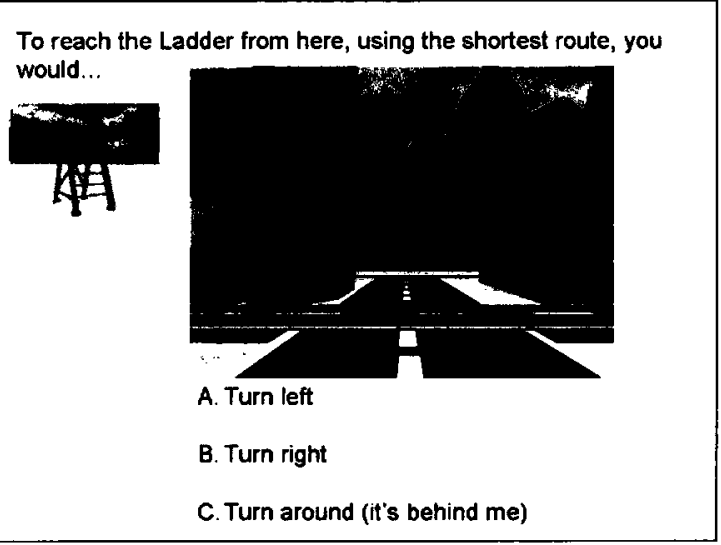


Experiment 1: Global-only group

\section{Question 1}

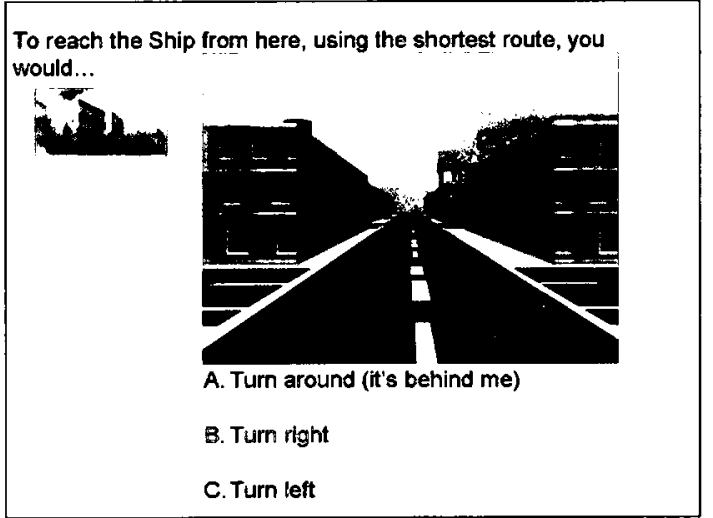

\section{Question 3}

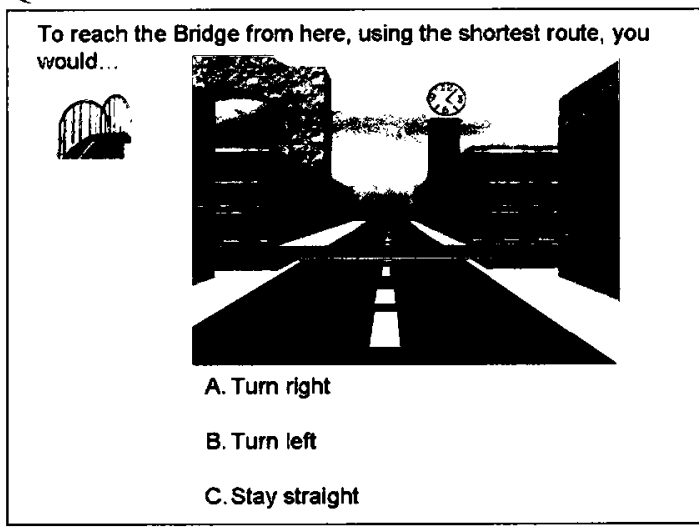

\section{Question 5}

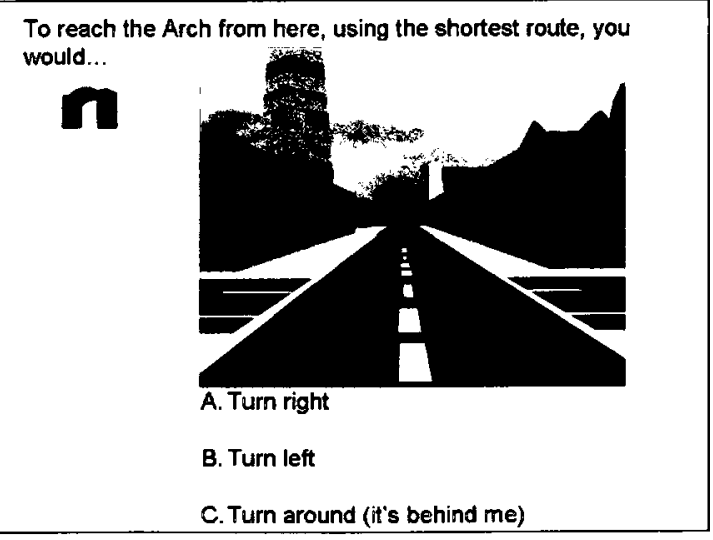

\section{Question 2}

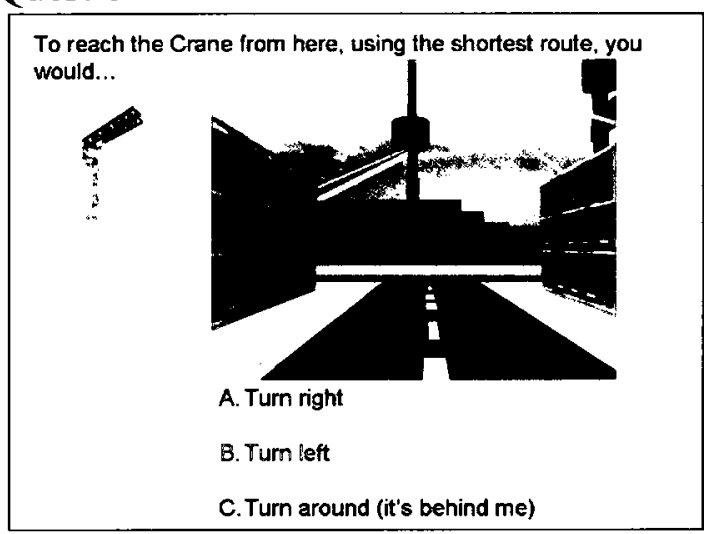

Question 4

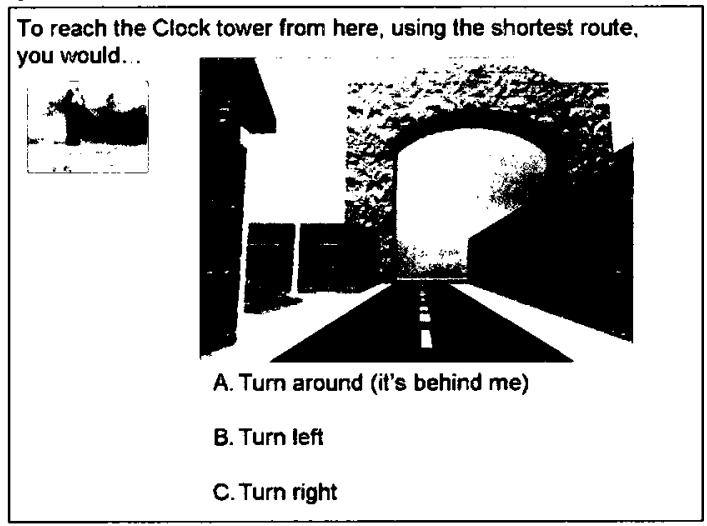

Question 6

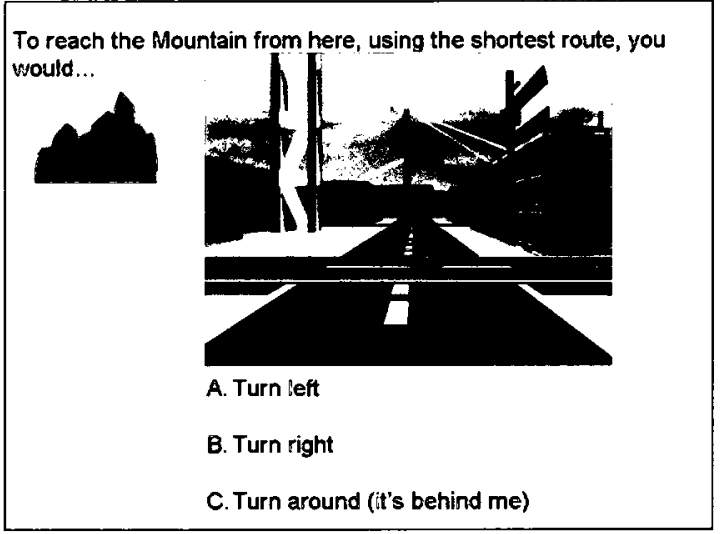




\section{Question 7}

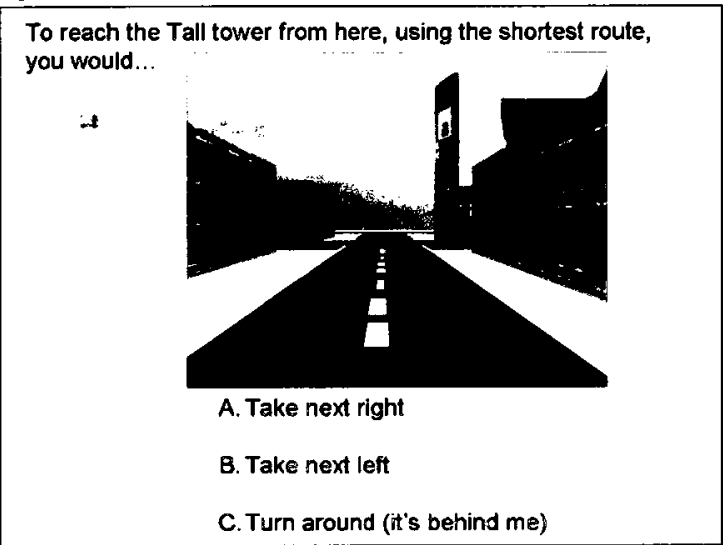

\section{Question 9}

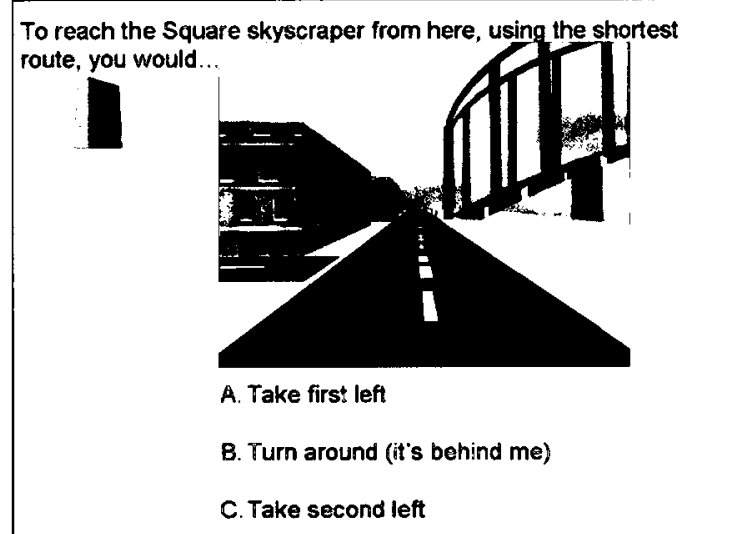

Question 11

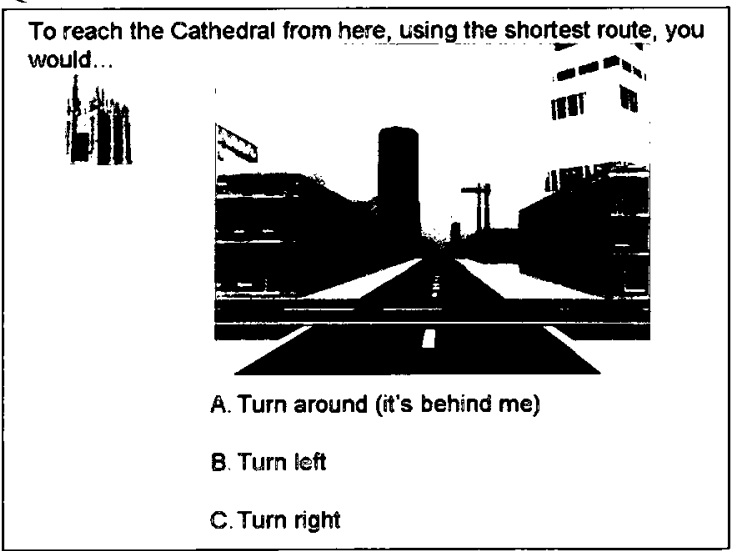

Question 8

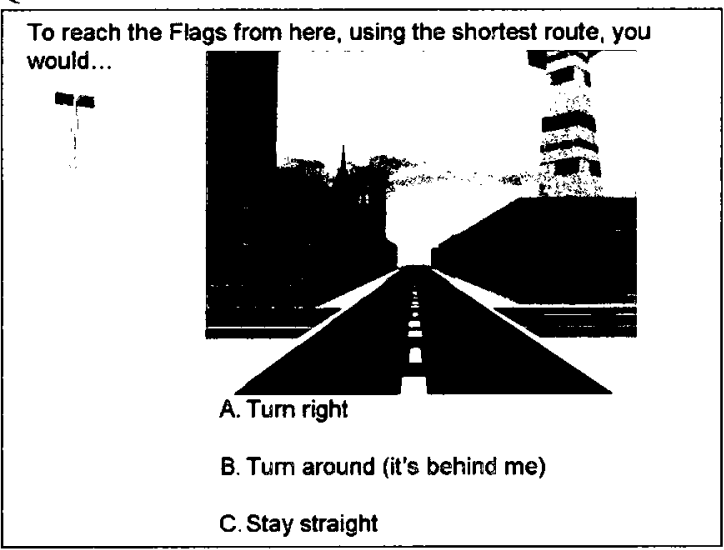

\section{Question 10}

To reach the Stadium from here, using the shortest route, you would..

Aromef

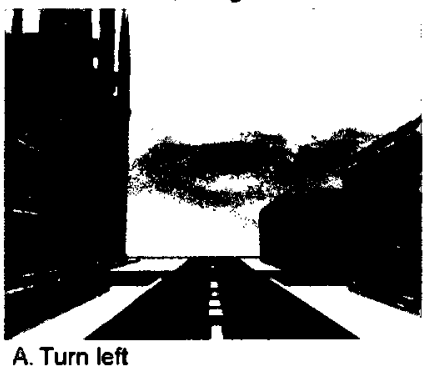

B. Turn right

C. Turn around (it's behind me)

\section{Question 12}

To reach the Factory from here, using the shortest route, you would...
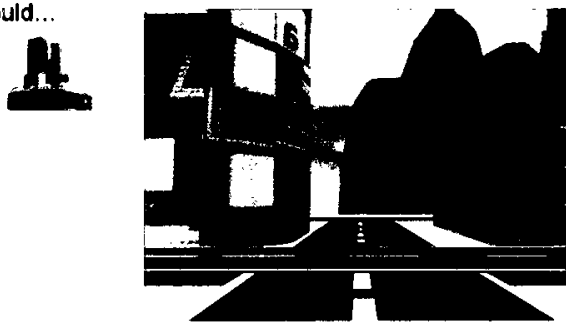
A. Turn teft
B. Turn right
C. Turn around (it's behind me) 
Experiment 1: Local-only group

\section{Question 1}

To reach the Vending machine from here, using the shortest route, you would.

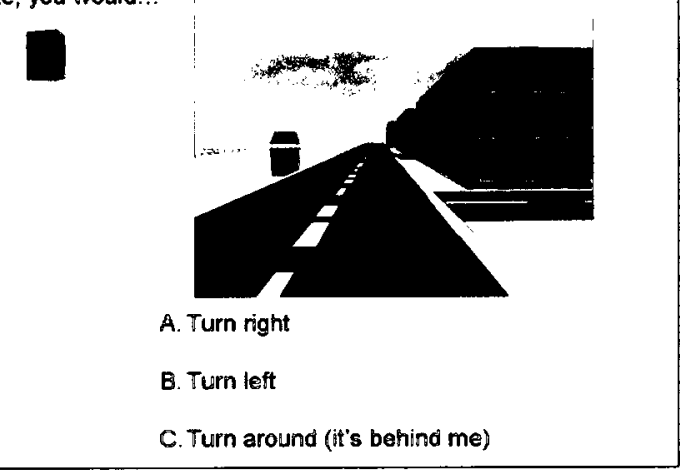

\section{Question 3}

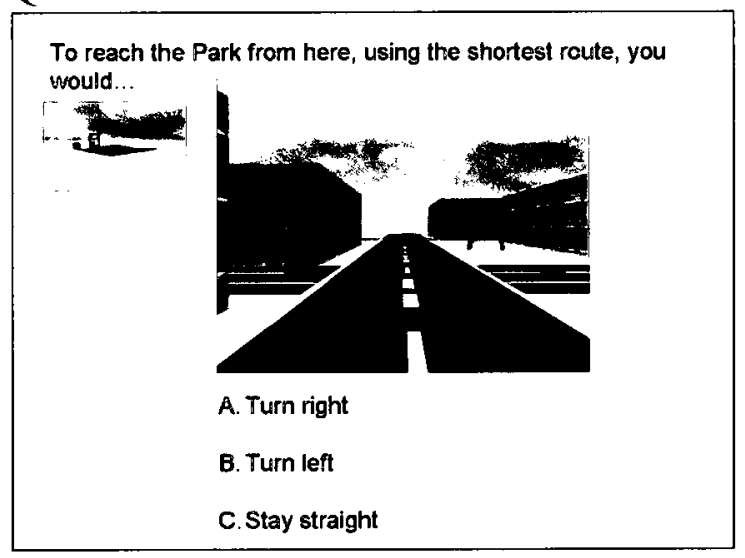

\section{Question 5}

To reach the Traffic light from here, using the shortest route, you would...
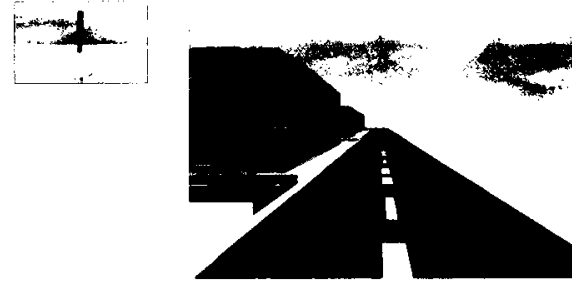

A. Take first left

8. Take second left

C. Turn around (it's behind me)
Question 2

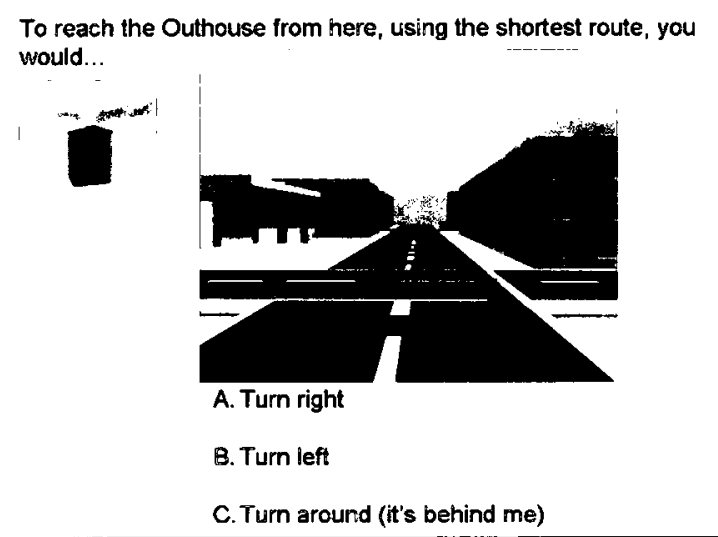

\section{Question 4}

To reach the Tennis court from here, using the shortest route, you would..
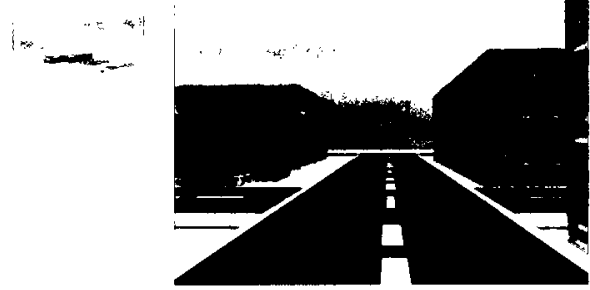

A. Turn right

B. Tum left

C. Turn around (it's behind me)

\section{Question 6}

To reach the Construction cones from here, using the shortest route, you would..

13

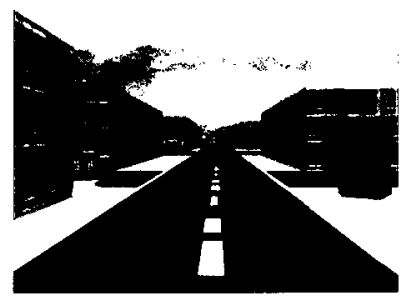

A. Turn right

B. Turn left

C. Tum around (it's behind me) 


\section{Question 7}

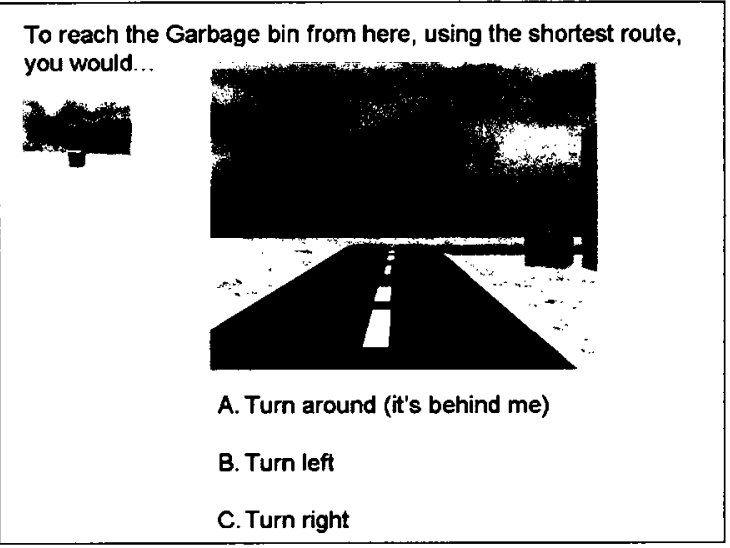

\section{Question 9}

To reach the Heli-pad from here, using the shortest route, you would...

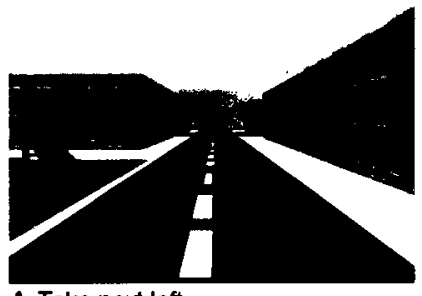

A. Take next left

\section{B. Take next right}

C. Turn around (it's behind me)

\section{Question 11}

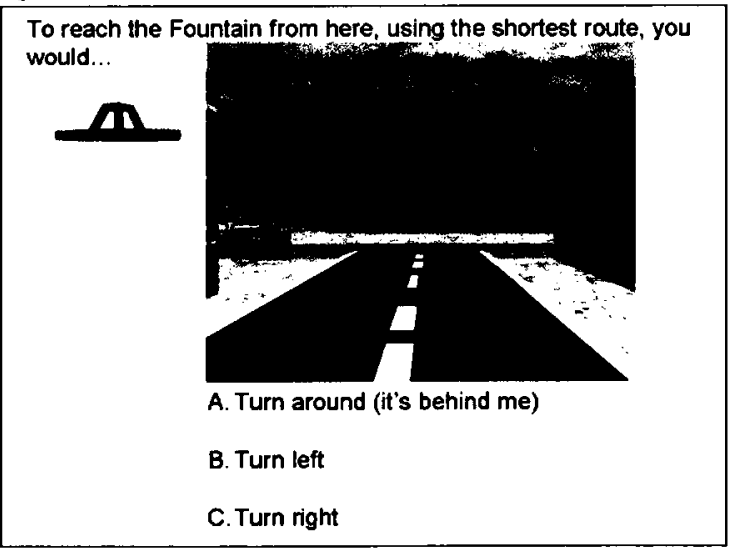

\section{Question 8}

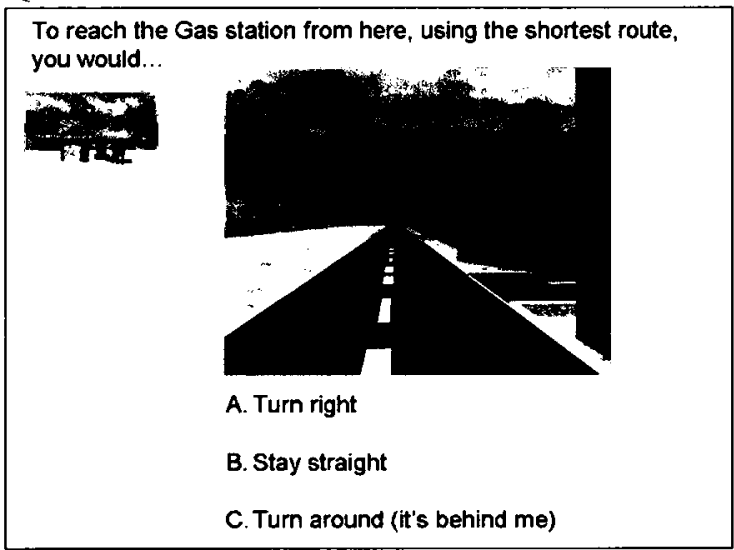

Question 10

To reach the Pool from here, using the shortest route, you would..
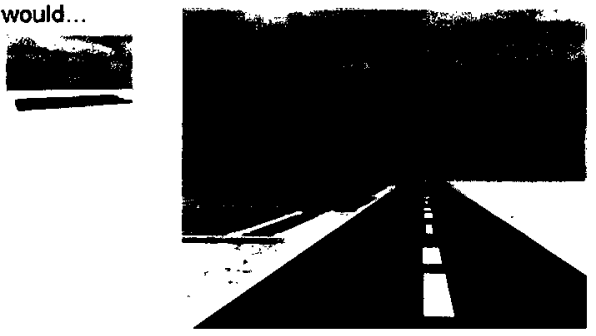

A. Turn around (it's behind me)

B Take first left

C. Take second left

\section{Question 12}

To reach the Ladder from here, using the shortest route, you would...
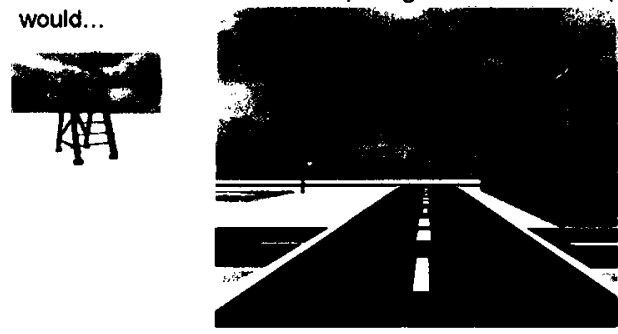

A. Turn left

B. Turn right

C. Turn around (it's behind me) 
Experiment 2: Peripheral group - global landmarks only

\section{Question 1}
A. Tum around tt's behind me
B. Tum right
C. Tum left

\section{Question 3}

To reach the Bridge from here, using the shortest route, you would...

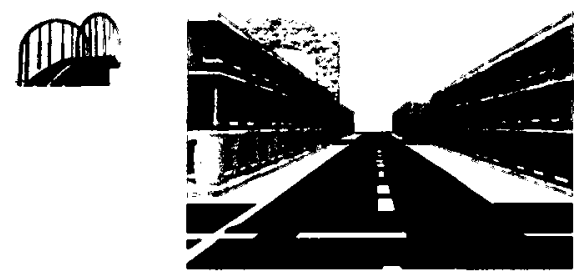
A. Tum right
B. Turn left
C. Stay stralght

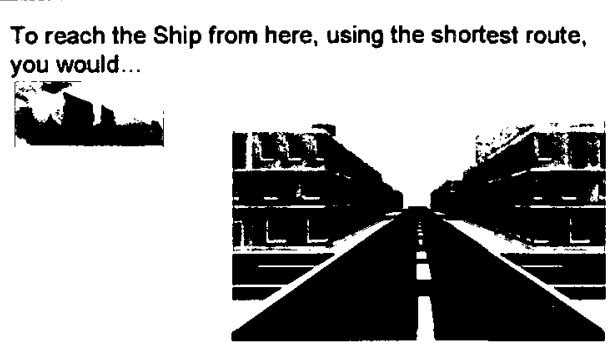

\section{Question 2}

To reach the Mountain from here, using the shortest route, you would...
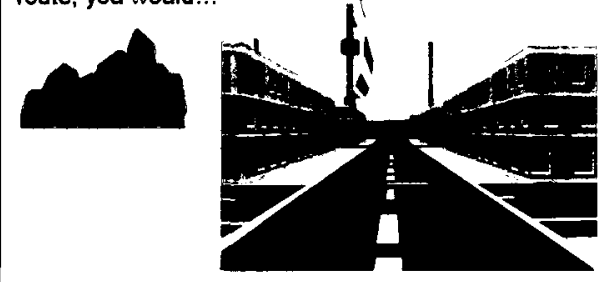
A. Turn left
B. Tum right
C. Turn around it's behind me

\section{Question 4}

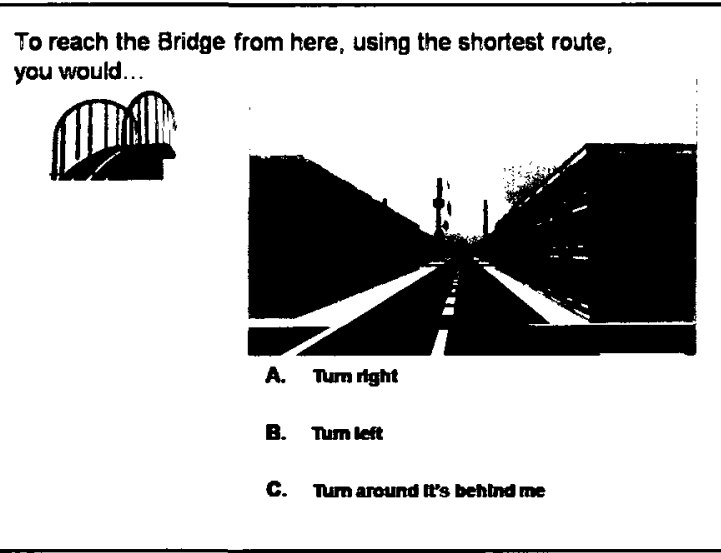

\section{Question 5}

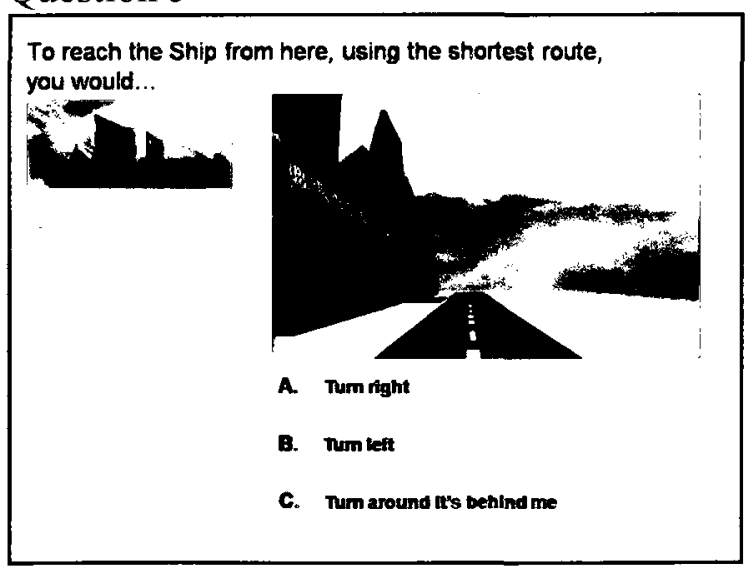

\section{Question 6}

To reach the Arch from here, using the shortest route, you would...

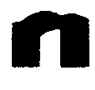
A. Tum night
B. numieft
C. Tum around tr's behtind me 
Question 7

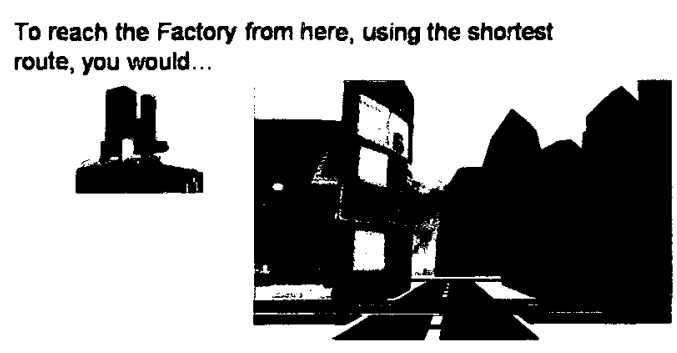
A. Tum around it's behind me
B. Tumber
c. Tum right

\section{Question 9}

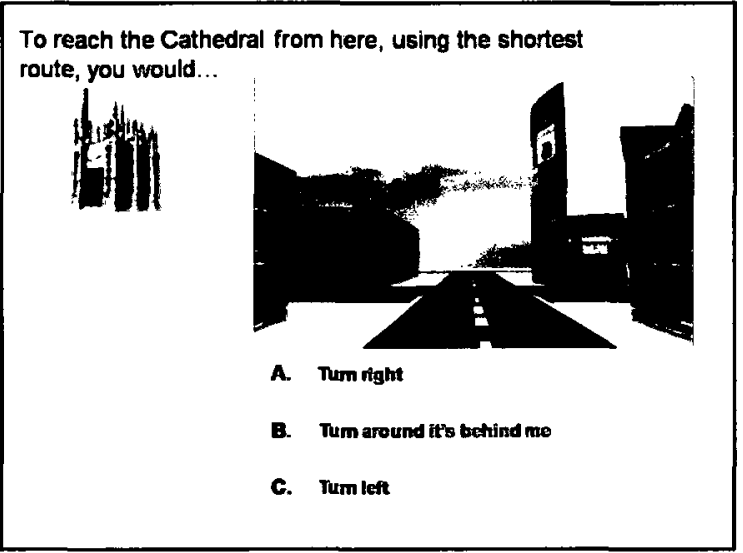

\section{Question 11}

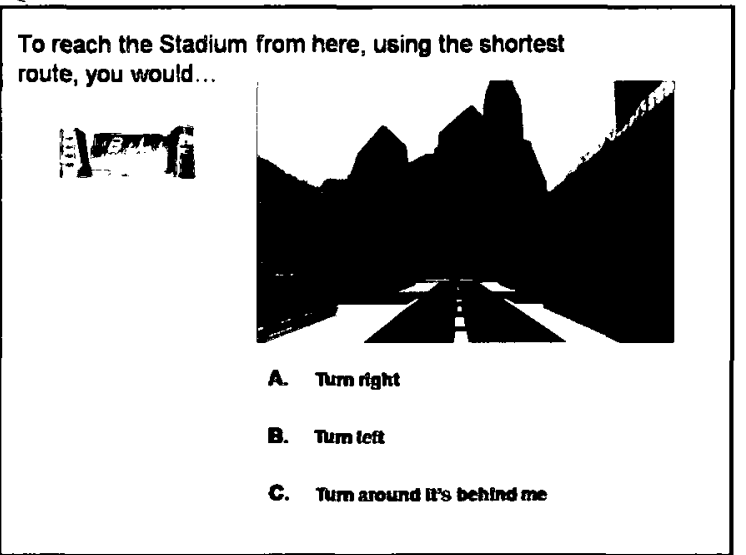

Question 8

To reach the Water Tower from here, using the shortest route, you would..
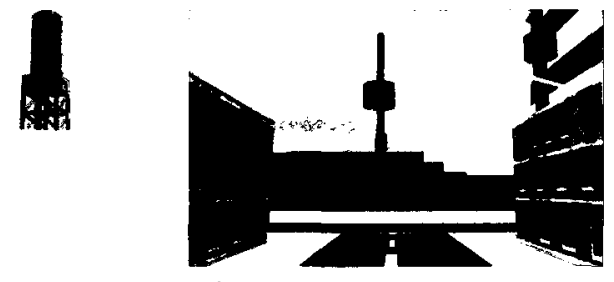
A. Tum right
B. Tum teft
C. Turn anound t's behind me

\section{Question 10}

To reach the Square Skyscraper from here, using the shortest route, you would...
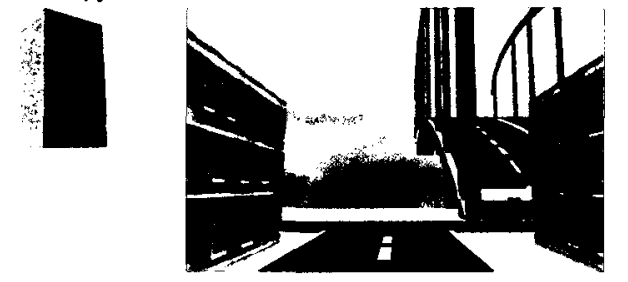
A. num right
B. Tim left
C. num around it's bentind me

\section{Question 12}

To reach the Arch from here, using the shortest route, you would..

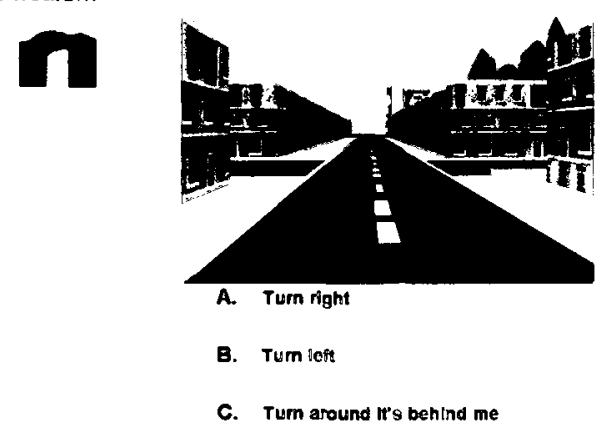


Experiment 2: Peripheral group - local

\section{Question 1}

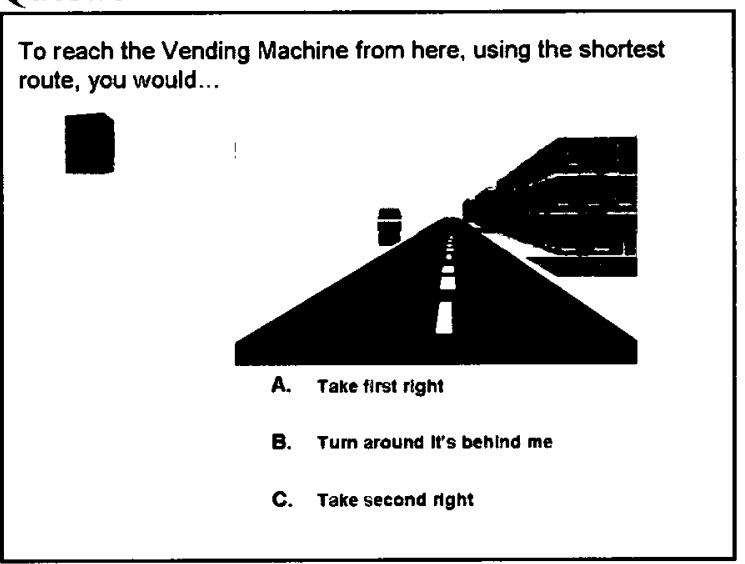

\section{Question 3}

To reach the Garbage Can from here, using the shortest route, you would.
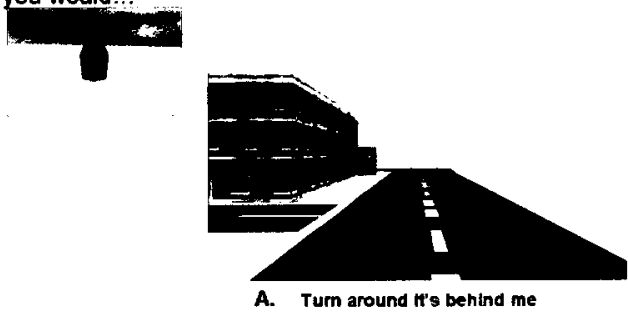

B. Take first Ieft

C. Take second left

\section{Question 5}

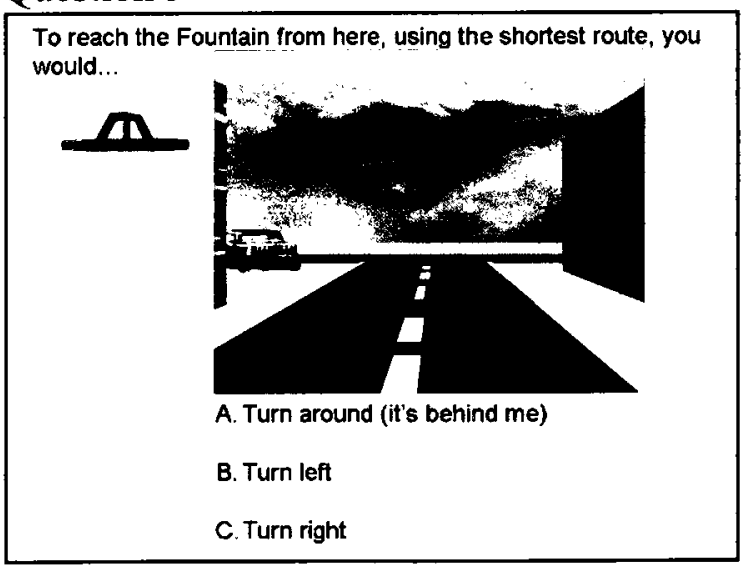

Question 2

To reach the Tennis Court from here, using the shortest route, you would..

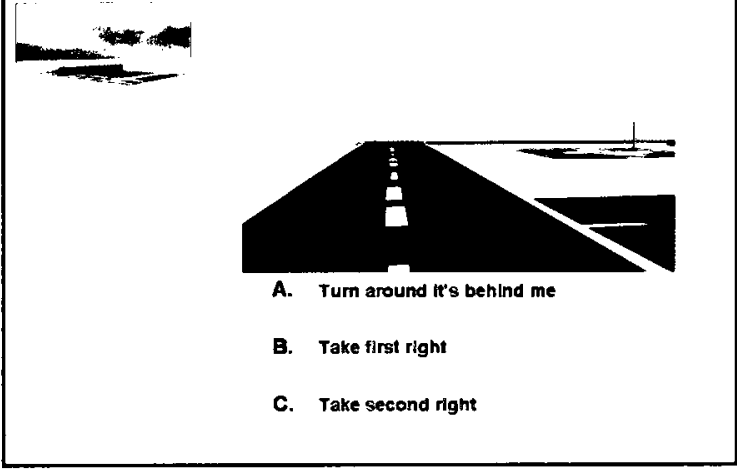

\section{Question 4}

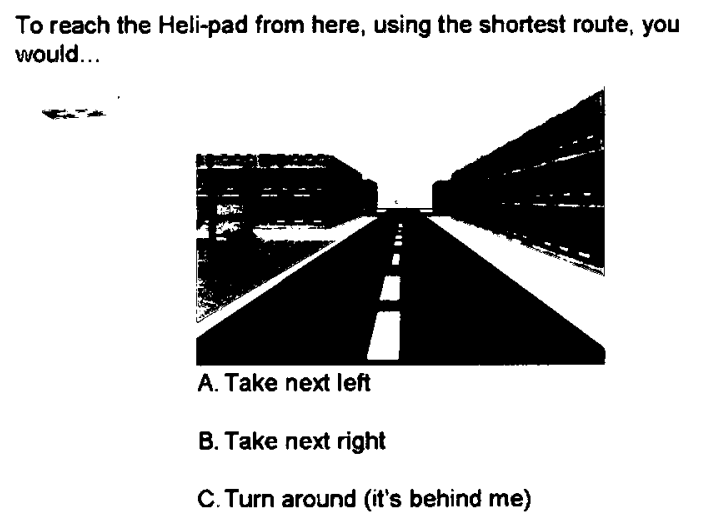

Question 6

To reach the Truck from here, using the shortest route, you would...

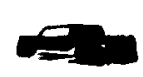
A. Turn around It's behtnd me
B. Tum left
c. Tum right 


\section{Question 7}

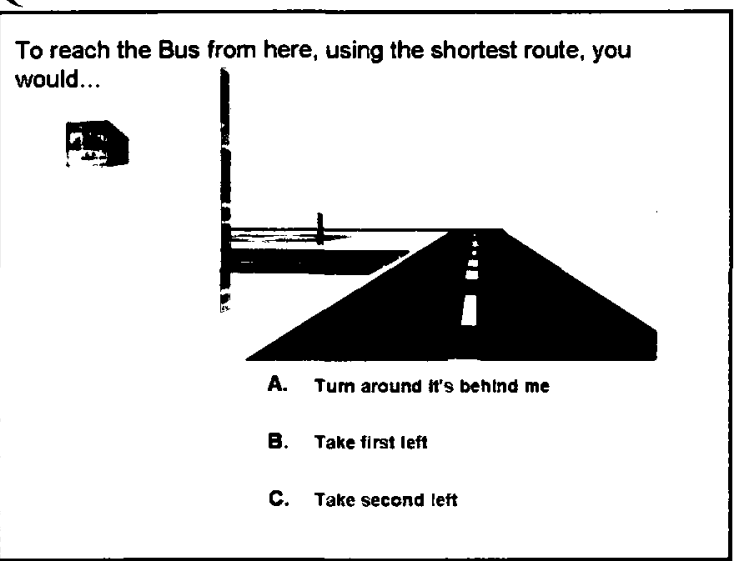

\section{Question 9}

To reach the Heli-pad from here, using the shortest route, you would..

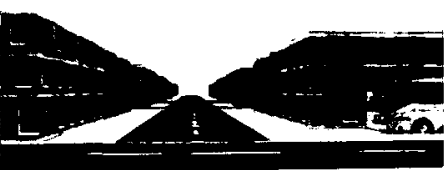
A. Keep stratght
B. Turn right
C. Tum leit

\section{Question 8}

To reach the Vending Machine from here, using the shortest route, you would.

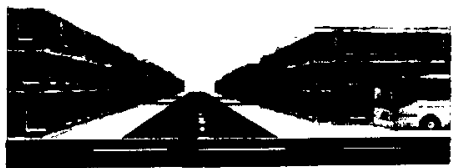
A. Keep straight
B. Turn right
c. Turn left

\section{Question 10}

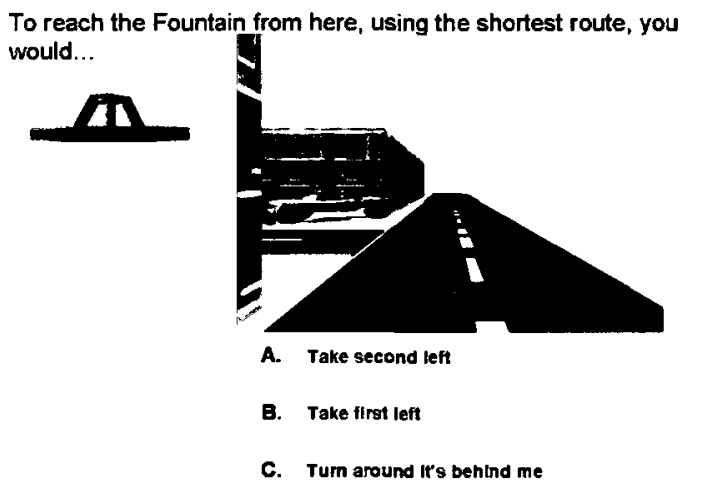

\section{Question 11}

To reach the Bus from here, using the shortest route, you would..
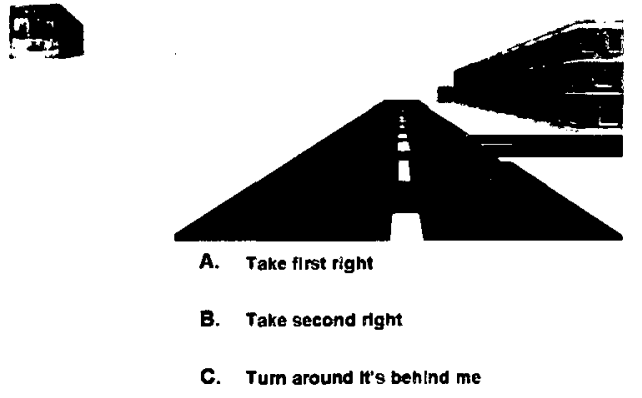

\section{Question 12}

To reach the Tennis Court from here, using the shortest route, you would.

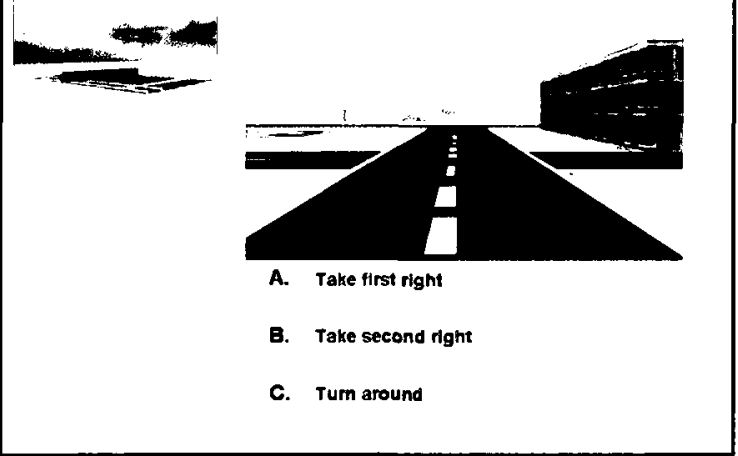


Experiment 2: Internal group - global

\section{Question 1}

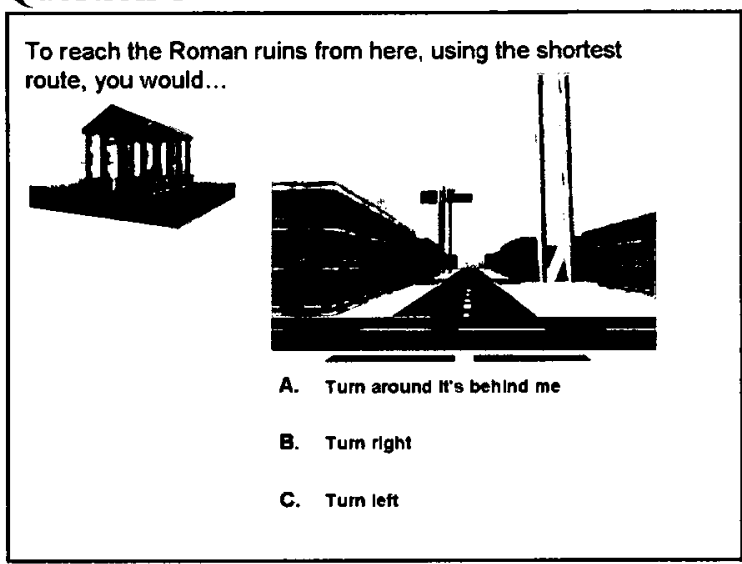

\section{Question 3}

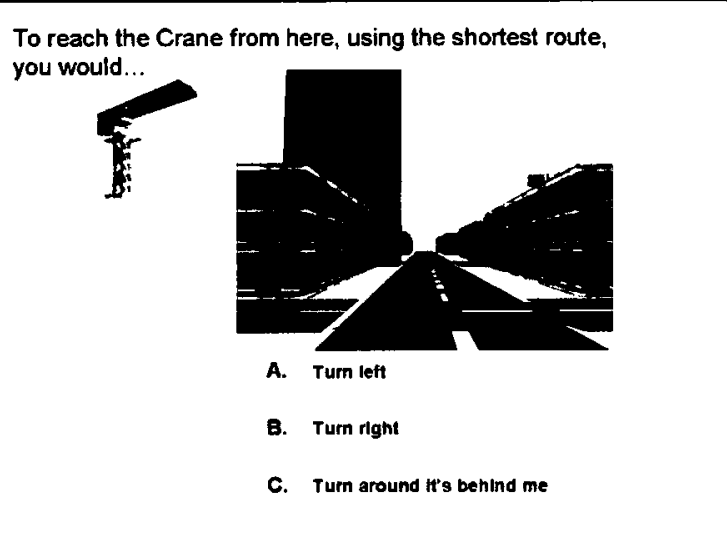

\section{Question 5}

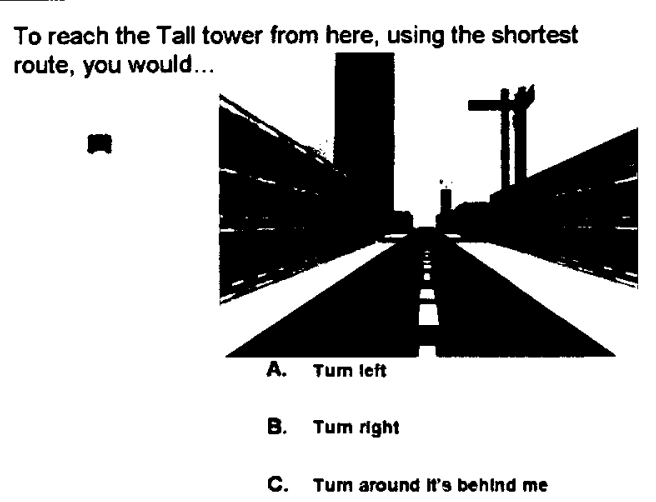

\section{Question 2}

To reach the Round skyscraper from here, using the shortest route, you would...

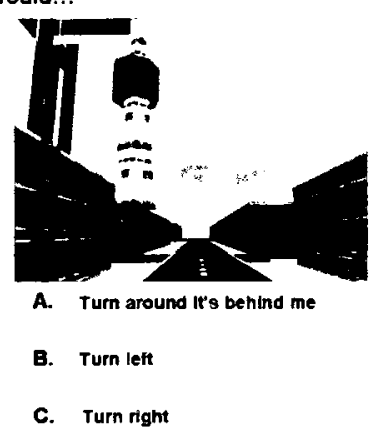

Question 4

To reach the Flags from here, using the shortest route, you would...
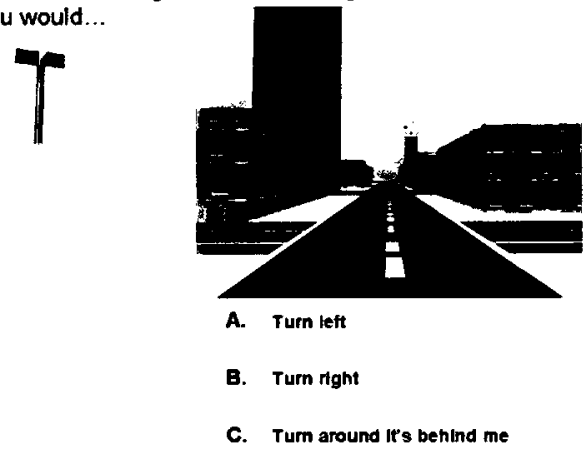

Question 6

To reach the Clock tower from here, using the shortest route, you would...
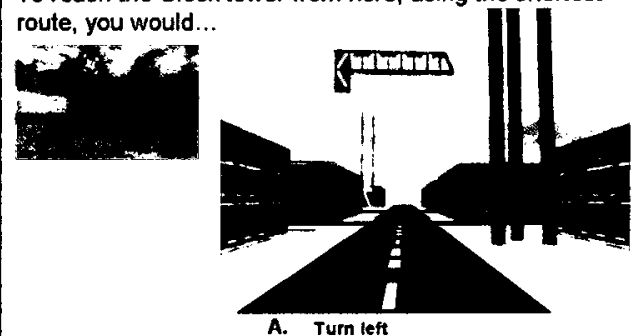
B. Turn right
C. Turn around It's behind me 


\section{Question 7}

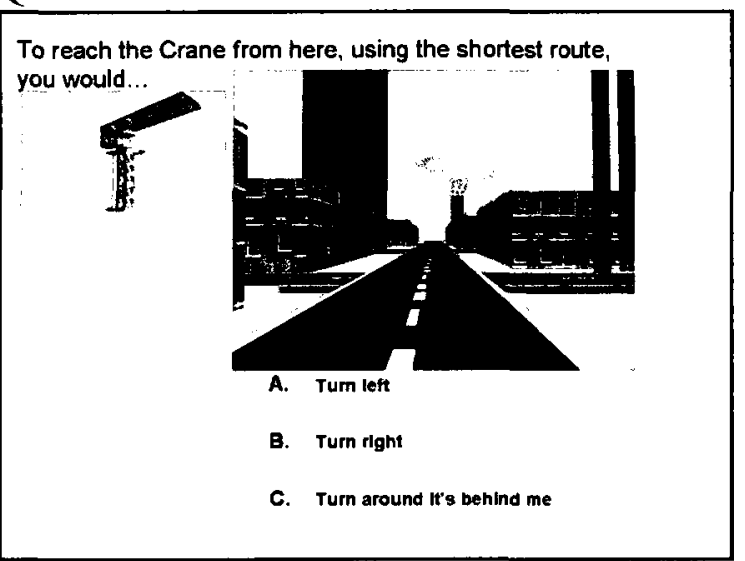

\section{Question 9}

To reach the Round skyscraper from here, using the shortest route, you would..
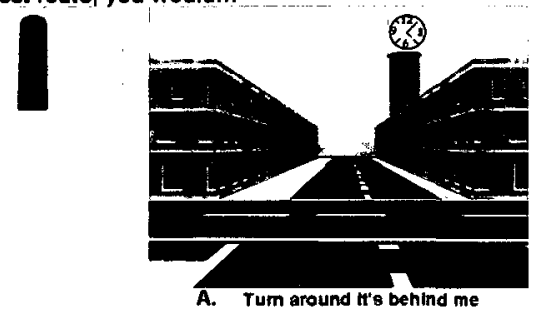
8. Tum left
C. Turn right

\section{Question 11}

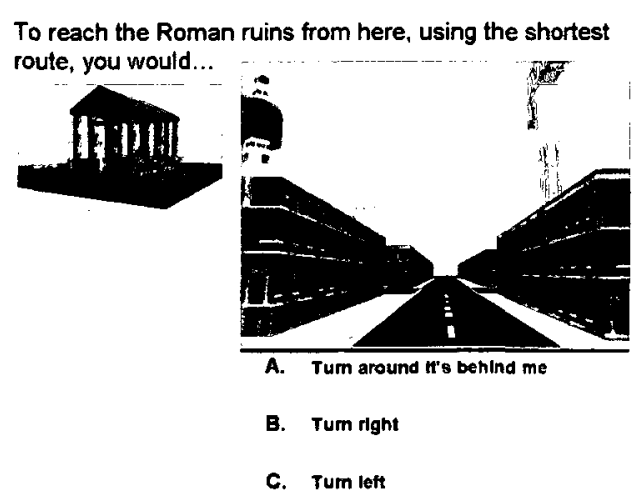

\section{Question 8}

To reach the Flags from here, using the shortest route you would..
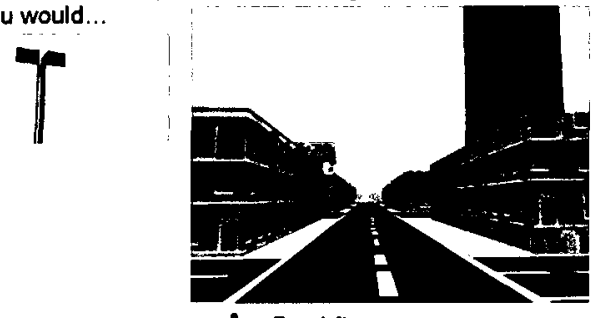
A. Turn left
B. Turn right
C. Turn around It's behind me

\section{Question 10}

To reach the Tall tower from here, using the shortest route, you would...

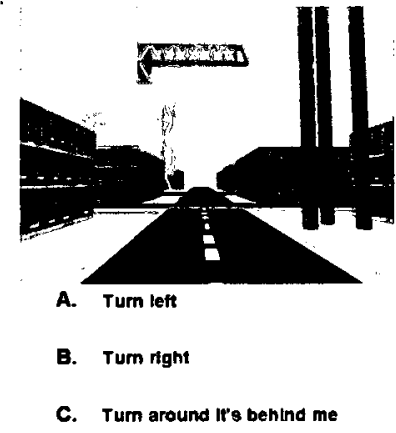

\section{Question 12}

To reach the Clock tower from here, using the shortest route, you would...
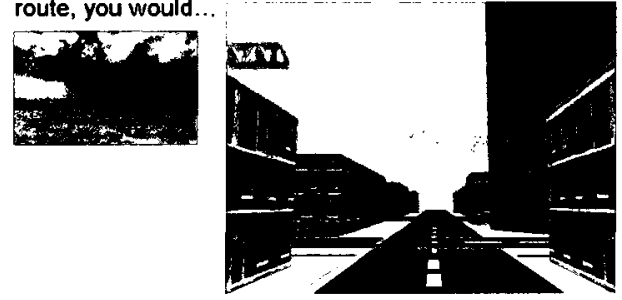
A. Turn leit
B. Turn right
C. Turn around in's behind me 
Experiment 2: Internal group - local

\section{Question 1}

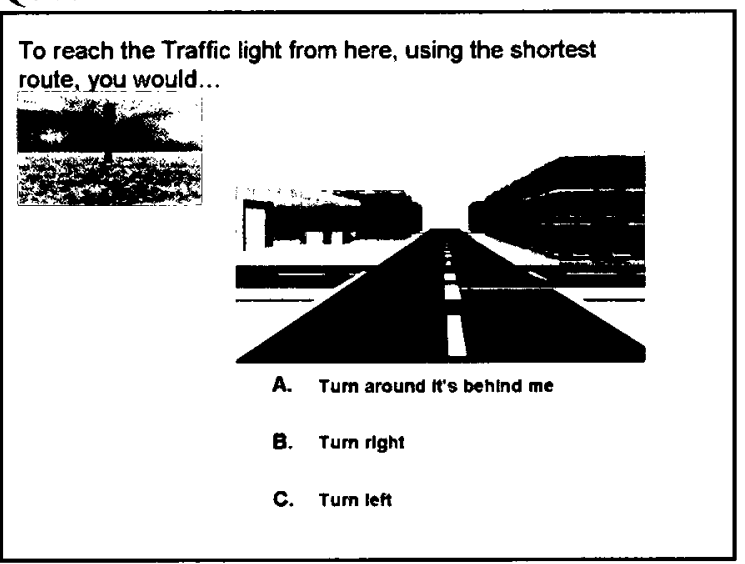

\section{Question 3}

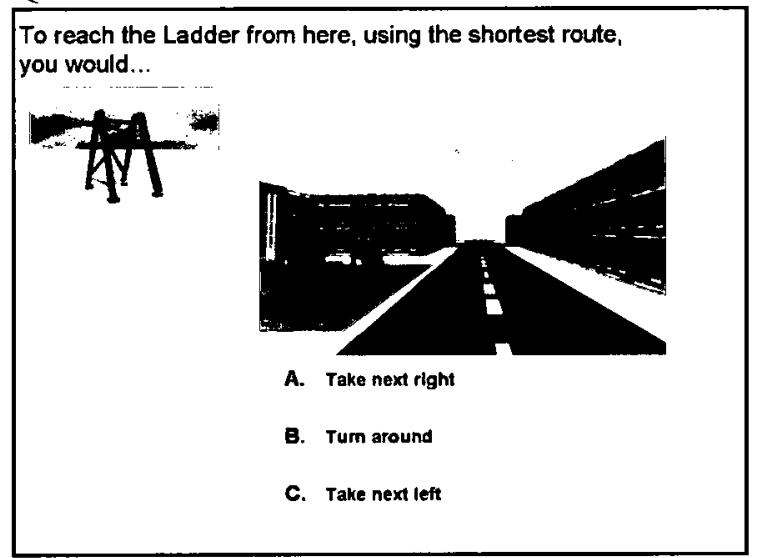

\section{Question 5}

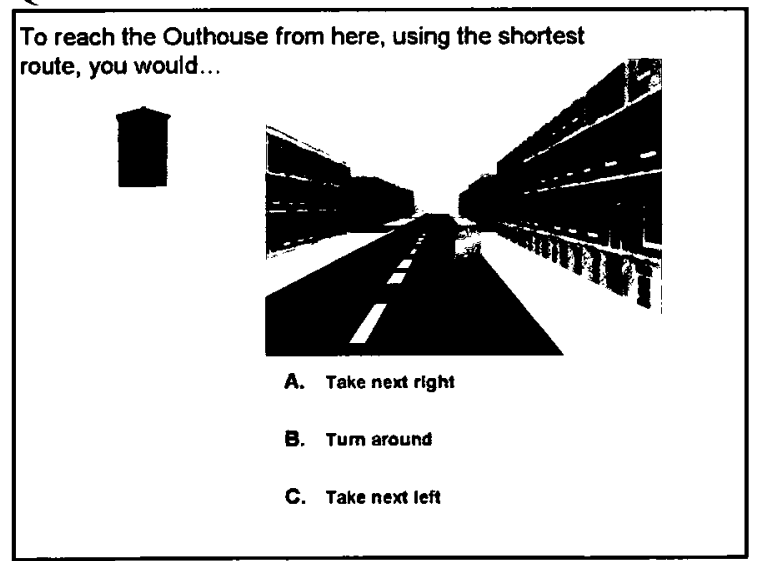

Question 2

To reach the Construction cones from here, using the shortest route, you would...

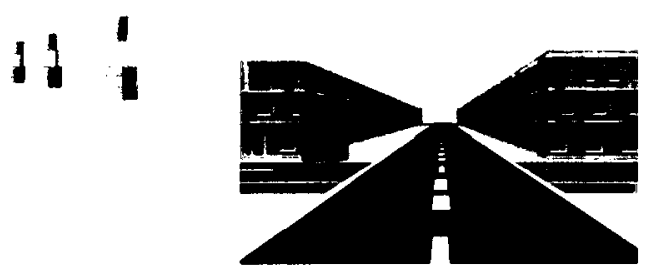

A. Turn around

B. Tum right

c. Tum left

Question 4

To reach the Parking sign from here, using the shortest route, you would.
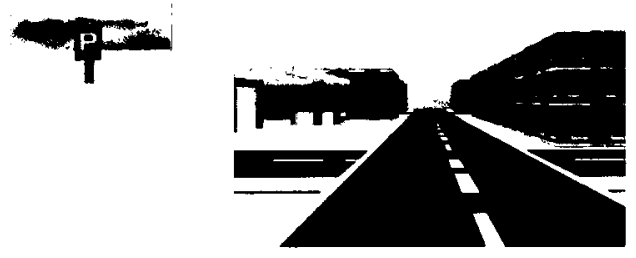
A. Turn fight
B. Tum around
C. Tum left

Question 6

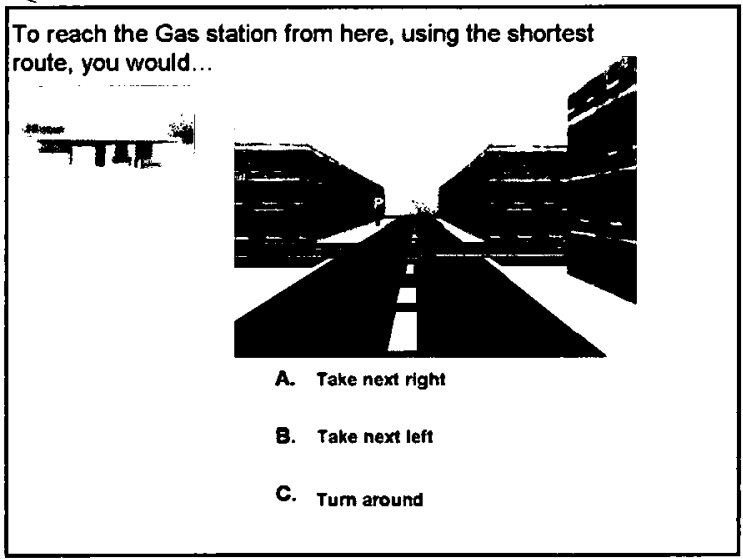




\section{Question 7}

To reach the Park from here, using the shortest route, you would...

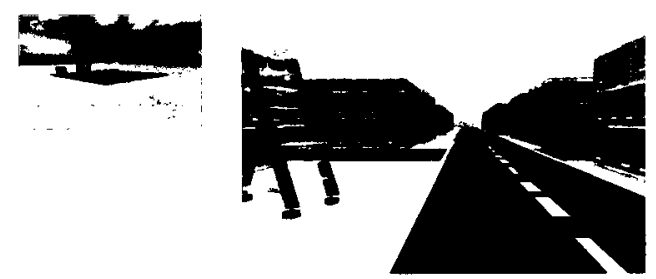
A. Take next right
B. Take next left
C. Tum around

\section{Question 9}

To reach the Parking sign from here, using the shortest route, you would..
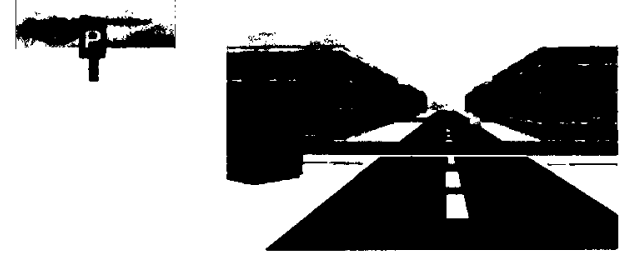
A. Tum right
B. Tum around
C. Tum left

\section{Question 8}

To reach the Pool from here, using the shortest route, you would...

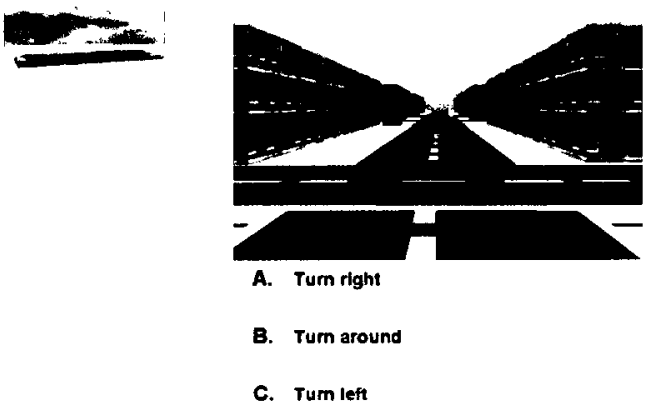

\section{Question 10}

To reach the Ladder from here, using the shortest route, you would..
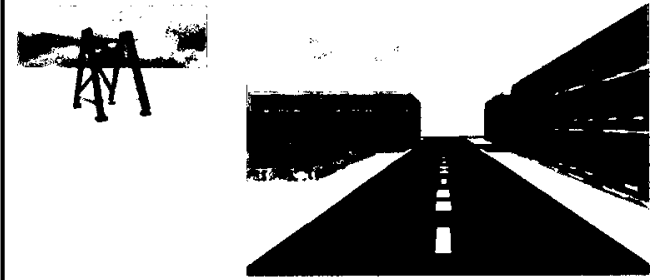
A. Take next right
B. Tum around
C. Take next teft

\section{Question 11}

To reach the Park from here, using the shortest route. you would...

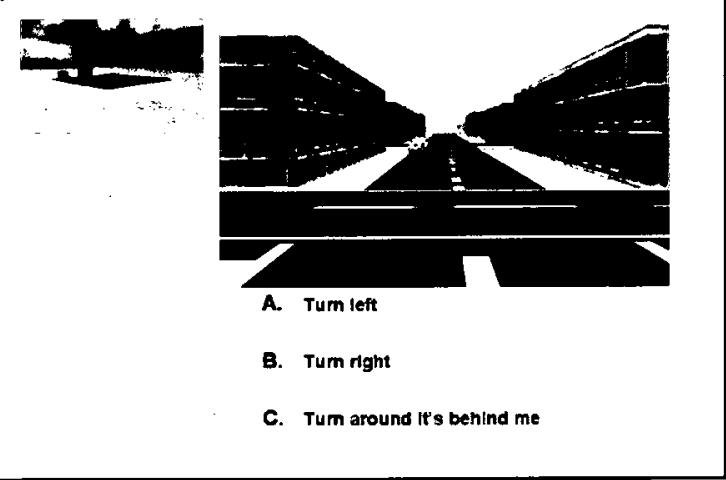

\section{Question 12}

To reach the Traffic light from here, using the shortest route, you would...
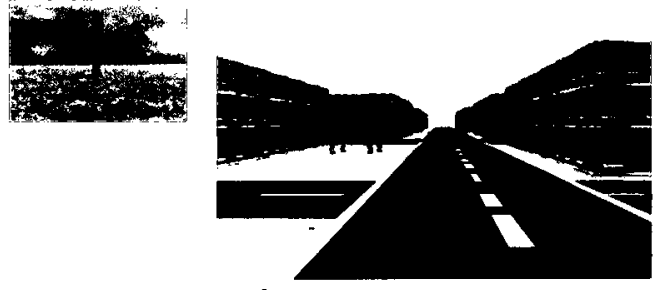
A. Tum left
B. Turn right
C. Tum around if $s$ behind me 
Appendix K. Spatial Strategies Questionnaire

\begin{tabular}{|l|l|l|}
\hline \multicolumn{1}{|c|}{ Orientation and Navigation questions } & True & False \\
\hline I kept track of the direction in which I was going. & & \\
entered the world. & & \\
\hline I thought of my location in the world in terms of North, & & \\
South, East or West. & & \\
Whenever I made a turn, I knew which direction I was & & \\
\hline I kept track of where I was in relation to a reference & & \\
point such as the centre, water, a specific object etc & & \\
\hline As I went I made a mental note of the distance I & & \\
traveled on different roads & & \\
\hline I relied heavily on the map as I went & & \\
\hline Objects in the world were helpful to me to find my way & & \\
\hline Objects in the world were helpful to me to section the & & \\
environment or find different areas within the world & & \\
\hline I counted the number of roads I passed to help me find & & \\
\hline
\end{tabular}


Appendix L. Experiment 1 - Way-finding Descriptive Statistics

Time to locate targets:

\begin{tabular}{lllllll} 
& \multicolumn{2}{l}{ Block 1 } & \multicolumn{2}{c}{ Block 3 } & \multicolumn{2}{l}{ Block 3 } \\
\cline { 2 - 7 } Group & \multicolumn{1}{c}{ M } & SD & M & SD & M & SD \\
\hline Both & 5.025 & .280 & 4.878 & .459 & 4.524 & .560 \\
Global-only & 4.965 & .217 & 4.796 & .425 & 4.743 & .475 \\
Local-only & 5.129 & .237 & 4.783 & .388 & 4.488 & .422 \\
\hline
\end{tabular}

Number of targets located:

\section{$\begin{array}{lll}\text { Block } 1 & \text { Block } 3 & \text { Block } 3\end{array}$}

\begin{tabular}{lcccccc}
\cline { 2 - 7 } Group & $\mathrm{M}$ & $\mathrm{SD}$ & $\mathrm{M}$ & $\mathrm{SD}$ & $\mathrm{M}$ & $\mathrm{SD}$ \\
\hline Both & 3.700 & .657 & 3.950 & 1.146 & 4.500 & .827 \\
Global-only & 4.100 & .968 & 4.250 & .967 & 4.150 & .933 \\
Local-only & 3.550 & .887 & 4.450 & .760 & 4.700 & .470 \\
\hline
\end{tabular}

Time spent way-finding as a function of location:

\begin{tabular}{lcccc} 
& \multicolumn{2}{c}{ Peipheral } & \multicolumn{2}{c}{ Internal } \\
\cline { 2 - 5 } Group & $\mathrm{M}$ & $\mathrm{SD}$ & $\mathrm{M}$ & $\mathrm{SD}$ \\
\hline Both & 5.882 & .483 & 6.750 & .478 \\
Global-only & 5.899 & .468 & 6.474 & .369 \\
Local-only & 5.785 & .465 & 6.515 & .281 \\
\hline
\end{tabular}




\section{Appendix M. Experiment 1 - Sketch Maps: Descriptive Statistics}

Ratio of the number of landmarks included in sketch maps to the total available:

\begin{tabular}{llllllll} 
& \multicolumn{2}{c}{ Total } & \multicolumn{3}{c}{ Peripheral } & \multicolumn{2}{c}{ Internal } \\
\cline { 2 - 7 } & & & & & & \\
\hline Both & .407 & .175 & .529 & .191 & .285 & .192 \\
Global-only & .727 & .268 & .772 & .218 & .558 & .321 \\
Local-only & .670 & .199 & .757 & .250 & .594 & .176 \\
\hline
\end{tabular}

Relative landmark placement:

\begin{tabular}{lcc} 
Group & $\mathrm{M}$ & $\mathrm{SD}$ \\
\hline Both & .770 & .249 \\
Global-only & .629 & .303 \\
Local-only & .533 & .336 \\
\hline
\end{tabular}

ANOVA table for tests of between group differences for relative landmark placement.

\begin{tabular}{lrccc} 
& df & MS & F & Sig. \\
\hline Between Groups & 2 & .285 & 3.204 & .048 \\
Within Groups & 57 & .089 & & \\
Total & 59 & & & \\
\hline
\end{tabular}

Appendix N. Experiment 1 - WDT Descriptive Statistics 
WDT descriptive statistics for the total number (regardless of landmark location) as well as by location.

\begin{tabular}{lcccccc} 
& \multicolumn{2}{c}{ Total } & \multicolumn{2}{c}{ Peripheral } & \multicolumn{2}{l}{ Internal } \\
\cline { 2 - 7 } \multicolumn{1}{c}{ Group } & $\mathrm{M}$ & $\mathrm{SD}$ & $\mathrm{M}$ & $\mathrm{SD}$ & $\mathrm{M}$ & $\mathrm{SD}$ \\
\hline Both & .468 & .244 & .497 & .258 & .509 & .249 \\
Global-only & .507 & .210 & .475 & .234 & .550 & .282 \\
Local-only & .600 & .154 & .690 & .220 & .536 & .160 \\
\hline
\end{tabular}

WDT descriptive statistics for all combinations of landmark type and location for the Both group.

\begin{tabular}{lcccccccc} 
& \multicolumn{2}{c}{ GP } & \multicolumn{2}{c}{ GI } & \multicolumn{2}{c}{ LP } & \multicolumn{2}{c}{ LI } \\
\cline { 2 - 9 } Group & M & SD & M & M & SD & SD & M & SD \\
\hline Both & 0.417 & .317 & 0.441 & .276 & 0.603 & .311 & 0.556 & .347 \\
\hline
\end{tabular}


Appendix O. Experiment 2 - Way-finding Descriptive Statistics

Way-finding descriptive statistics for Ln time(s) to locate targets as a function of blocks:

\begin{tabular}{|c|c|c|c|c|c|c|c|c|}
\hline \multirow[b]{3}{*}{ Group } & \multicolumn{2}{|c|}{ Block 1} & \multicolumn{2}{|c|}{ Block 2} & \multicolumn{2}{|c|}{ Block 3} & \multicolumn{2}{|c|}{ Block 4} \\
\hline & \multicolumn{4}{|c|}{ Global } & \multicolumn{4}{|c|}{ Local } \\
\hline & $\mathrm{M}$ & SD & M & M & SD & SD & M & SD \\
\hline Peripheral - Order 1 & .778 & .219 & .893 & .149 & .809 & .175 & .905 & .185 \\
\hline \multirow[t]{2}{*}{ Internal - Order 1} & .783 & .277 & .880 & .183 & .859 & .166 & .848 & .181 \\
\hline & \multicolumn{4}{|c|}{ Local } & \multicolumn{4}{|c|}{ Global } \\
\hline Peripheral - Order 2 & .783 & .216 & .848 & .197 & .848 & .196 & .869 & .148 \\
\hline Internal - Order 2 & .817 & .202 & .788 & .147 & .813 & .213 & .888 & .172 \\
\hline
\end{tabular}

Way-finding descriptive statistics for number of targets located as a function of blocks:

\begin{tabular}{|c|c|c|c|c|c|c|c|c|}
\hline \multirow[b]{3}{*}{ Group } & \multicolumn{2}{|c|}{ Block 1} & \multicolumn{2}{|c|}{ Block 2} & \multicolumn{2}{|c|}{ Block 3} & \multicolumn{2}{|c|}{ Block 4} \\
\hline & \multicolumn{4}{|c|}{ Global } & \multicolumn{4}{|c|}{ Local } \\
\hline & M & SD & $\mathbf{M}$ & M & $\mathrm{SD}$ & $\mathrm{SD}$ & M & $\mathrm{SD}$ \\
\hline Peripheral - Order 1 & 5.039 & .414 & 4.698 & .384 & 4.860 & .4765 & 4.833 & .402 \\
\hline \multirow[t]{2}{*}{ Internal - Order 1} & 5.024 & .393 & 4.815 & .364 & 4.808 & .407 & 4.698 & .478 \\
\hline & \multicolumn{4}{|c|}{ Local } & \multicolumn{4}{|c|}{ Global } \\
\hline Peripheral - Order 2 & 4.914 & .489 & 4.866 & .301 & 4.746 & .550 & 4.737 & .420 \\
\hline Internal - Order 2 & 4.971 & .331 & 4.954 & .351 & 4.995 & .388 & 4.828 & .406 \\
\hline
\end{tabular}


Time spent way-finding as a function of location:

\begin{tabular}{lcccc} 
& \multicolumn{2}{c}{ Peripheral } & \multicolumn{2}{c}{ Internal } \\
\cline { 2 - 5 } Group & $\mathrm{M}$ & $\mathrm{SD}$ & $\mathrm{M}$ & $\mathrm{SD}$ \\
& & & & \\
\hline Peripheral - Order 1 & 5.025 & .451 & 5.912 & .291 \\
Internal - Order 1 & 5.073 & .328 & 5.879 & .296 \\
Peripheral - Order 2 & 4.881 & .315 & 5.906 & .237 \\
Internal - Order 2 & 5.079 & .310 & 5.992 & .169 \\
\hline
\end{tabular}


Appendix P. Experiment 2 - Sketch Maps: Descriptive Statistics

Ratio of the number of landmarks included in sketch maps to the total available:

\begin{tabular}{ccrrrrrrr} 
& \multicolumn{2}{c}{ Global } & \multicolumn{2}{c}{ Local } & \multicolumn{2}{c}{ Total } \\
\cline { 2 - 8 } Group & M & SD & M & SD & M & SD \\
Peripheral - Order 1 & .4698 & .10641 & .4063 & .08668 & .8214 & .12545 \\
Internal-Order 1 & .3206 & .08061 & .4091 & .10944 & .7662 & .19615 \\
\hline
\end{tabular}

$\begin{array}{llllllll}\text { Peripheral - Order } 2 & .4667 & .14071 & .3884 & .13432 & .8050 & .23610 \\ \text { Internal - Order } 2 & .3133 & .08122 & .3206 & .14239 & .6885 & .20311\end{array}$

Relative landmark placement descriptive statistics:

\begin{tabular}{ccccc} 
& \multicolumn{2}{c}{ Global } & \multicolumn{2}{c}{ Local } \\
\cline { 2 - 5 } Group & $\mathrm{M}$ & $\mathrm{SD}$ & $\mathrm{M}$ & $\mathrm{M}$ \\
\hline Peripheral - Order 1 & .9048 & .13593 & .9143 & .13522 \\
Internal - Order 1 & .8286 & .20284 & .7364 & .23411 \\
\cline { 2 - 6 } & & & Local & Global \\
Peripheral - Order 2 & .8435 & .26255 & .8870 & .19841 \\
Internal-Order 2 & .5714 & .27045 & .7800 & .24192 \\
\hline
\end{tabular}




\section{Map Goodness Ratings}

\begin{tabular}{lcccc} 
& \multicolumn{2}{c}{ Global } & \multicolumn{2}{c}{ Local } \\
\cline { 2 - 5 } Group & $\mathrm{M}$ & $\mathrm{SD}$ & $\mathrm{M}$ & $\mathrm{M}$ \\
\hline Peripheral - Order 1 & 3.524 & 1.020 & 3.318 & .997 \\
Internal - Order 1 & 2.381 & .570 & 2.778 & .884 \\
\cline { 2 - 6 } & \multicolumn{2}{c}{ Local } & Global \\
Peripheral - Order 2 & 2.797 & .973 & 3.304 & 1.091 \\
Internal - Order 2 & 2.649 & .899 & 2.526 & .660 \\
\hline
\end{tabular}




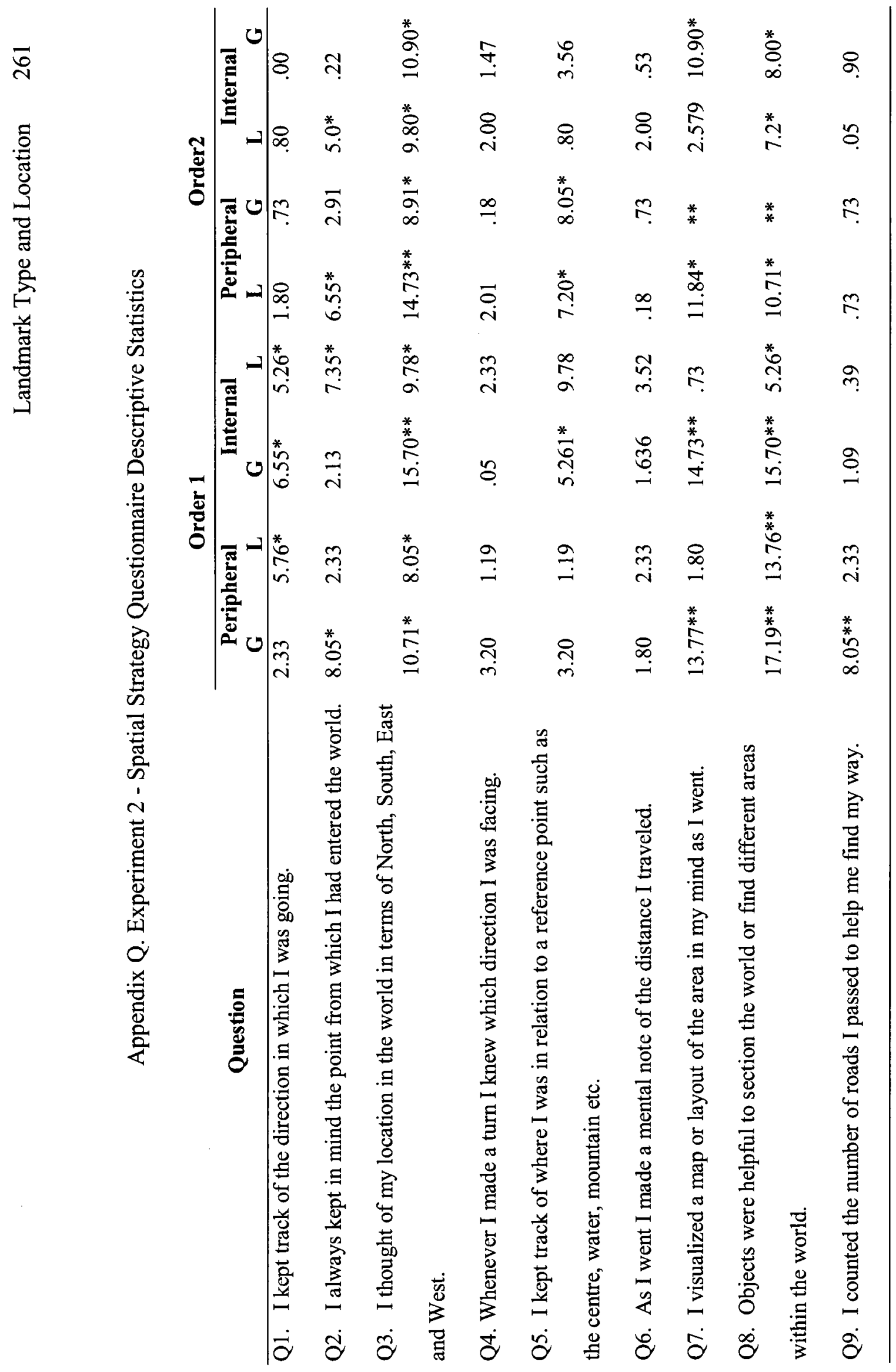


Appendix R. Experiment 2 - WDT Descriptive Statistics

\begin{tabular}{ccccc} 
Group & \multicolumn{2}{c}{ Global } & \multicolumn{2}{c}{ Local } \\
& $\mathrm{M}$ & $\mathrm{SD}$ & $\mathrm{M}$ & $\mathrm{M}$ \\
\hline Peripheral - Order 1 & .5595 & .25296 & .5794 & .15018 \\
Internal - Order 1 & .5036 & .21243 & .4674 & .16617 \\
\hline & \multicolumn{2}{c}{ Local } & \multicolumn{2}{c}{ Global } \\
Peripheral - Order 2 & .5326 & .21281 & .5833 & .22332 \\
Internal - Order 2 & .4000 & .14459 & .4000 & .18257 \\
\hline
\end{tabular}


Appendix S. Experiment 3 - Way-finding Descriptive Statistics

Time to locate targets:

\begin{tabular}{llccccc} 
& Total & \multicolumn{3}{c}{ Global } & \multicolumn{3}{c}{ Local } \\
\cline { 2 - 7 } Group & $\mathrm{M}$ & $\mathrm{SD}$ & $\mathrm{M}$ & $\mathrm{SD}$ & $\mathrm{M}$ & $\mathrm{SD}$ \\
\hline Novice & 9.736 & 4.931 & 5.789 & 2.740 & 3.947 & 2.857 \\
Intermediate & 11.578 & 5.367 & 6.947 & 3.390 & 4.631 & 2.692 \\
Advanced & 10.579 & 6.336 & 5.894 & 3.478 & 4.684 & 3.637 \\
\hline
\end{tabular}

Number of targets located:

\begin{tabular}{lllllll} 
& \multicolumn{2}{l}{ Block 1 } & \multicolumn{3}{l}{ Block 3 } & \multicolumn{2}{l}{ Block 3 } \\
\cline { 2 - 7 } Group & $\mathrm{M}$ & $\mathrm{SD}$ & $\mathrm{M}$ & $\mathrm{SD}$ & $\mathrm{M}$ & SD \\
\hline Novice & 1.250 & .967 & .750 & .966 & .550 & .884 \\
Intermediate & 1.158 & 1.015 & .631 & .597 & .841 & .834 \\
Advanced & 1.000 & .745 & .895 & 1.149 & .526 & .696 \\
Control & .750 & .638 & .600 & .753 & .300 & .656 \\
\hline
\end{tabular}


Time spent way-finding as a function of location:

\begin{tabular}{lllll} 
& \multicolumn{2}{l}{ Peripheral } & \multicolumn{2}{l}{ Internal } \\
\cline { 2 - 5 } Group & $\mathrm{M}$ & $\mathrm{SD}$ & $\mathrm{M}$ & $\mathrm{SD}$ \\
& & & & \\
\hline Novice & 5.5413 & .32695 & 6.5491 & .35515 \\
Intermediate & 5.4629 & .29112 & 6.4474 & .25961 \\
Advanced & 5.5230 & .62515 & 6.3469 & .48266 \\
\hline
\end{tabular}


Appendix T. Experiment 3 - Sketch Maps: Descriptive Statistics

Ratio of the number of landmarks included in sketch maps to the total available:

\begin{tabular}{lllllll} 
& Total & \multicolumn{3}{c}{ Global } & \multicolumn{3}{c}{ Local } \\
\cline { 2 - 7 } Group & $\mathrm{M}$ & $\mathrm{SD}$ & $\mathrm{M}$ & $\mathrm{SD}$ & $\mathrm{M}$ & $\mathrm{SD}$ \\
\hline Novice & 9.736 & 4.931 & 5.789 & 2.740 & 3.947 & 2.857 \\
Intermediate & 11.578 & 5.367 & 6.947 & 3.390 & 4.631 & 2.692 \\
Advanced & 10.579 & 6.336 & 5.894 & 3.478 & 4.684 & 3.637 \\
\hline
\end{tabular}

Relative landmark placement:

\section{Total}

\begin{tabular}{lll}
\cline { 2 - 3 } Group & $\mathrm{M}$ & $\mathrm{SD}$ \\
\hline Novice & .6300 & .26970 \\
Intermediate & .7579 & .27952 \\
Advanced & .6947 & .32910 \\
\hline
\end{tabular}


Appendix U. Experiment 3 - WDT Descriptive Statistics

\begin{tabular}{lllllll} 
& Total & \multicolumn{3}{c}{ Global } & \multicolumn{2}{c}{ Local } \\
\cline { 2 - 7 } Group & & & & & & \\
\hline Novice & .3583 & .13813 & .3417 & .16645 & .3714 & .16967 \\
Intermediate & .5526 & .18883 & .5175 & .18985 & .5865 & 18985 \\
Advanced & .4561 & .20669 & 4825 & .24780 & .4060 & .29432 \\
\hline
\end{tabular}

\begin{tabular}{lllll} 
& \multicolumn{3}{l}{ Peripheral } & \multicolumn{2}{l}{ Internal } \\
\cline { 2 - 5 } Group & $\mathrm{M}$ & $\mathrm{SD}$ & $\mathrm{M}$ & $\mathrm{SD}$ \\
\hline Novice & .3357 & .22836 & .4300 & 21788 \\
Intermediate & .5113 & .24888 & .6105 & .22582 \\
Advanced & 4825 & .30376 & .4632 & .22164 \\
\hline
\end{tabular}

